\title{
ROBUST AND DISTRIBUTED STATE ESTIMATION FOR POWER SYSTEMS
}

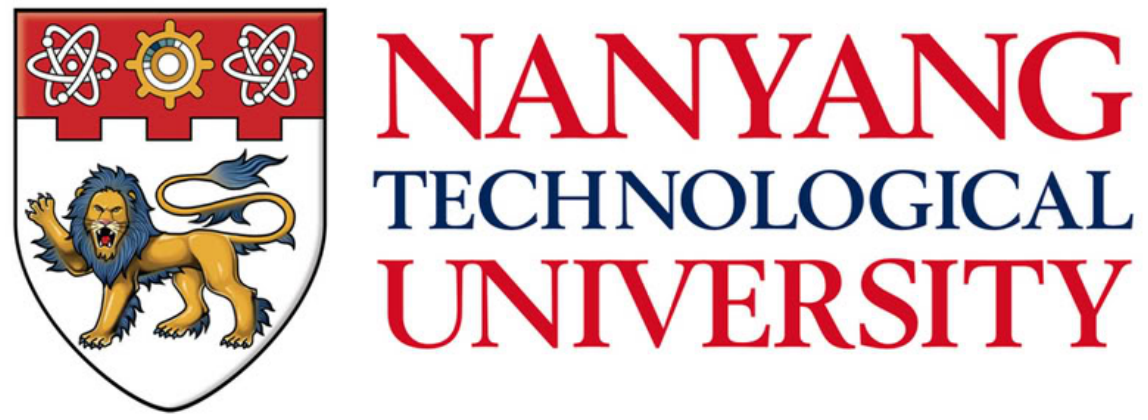

\section{CHEN TENGPENG}

School of Electrical \& Electronic Engineering

A thesis submitted to the Nanyang Technological University in fulfillment of the requirements for the degree of

Doctor of Philosophy 



\section{Statement of Originality}

I hereby certify that the work embodied in this thesis is the result of original research and has not been submitted for a higher degree to any other University or Institution. 



\section{Acknowledgement}

Firstly, I would like to express my heartiest gratitude to my supervisor, Associate Professor Ling Keck Voon, for his unconditional support, professional guidance, understanding and inspiration during my $\mathrm{PhD}$ study. Not only did his insights help to shape this work, but his aesthetic sense taught me what good research is truly about. The attitudes towards work I learned from him are logical, tolerant and careful, which will definitely be my valuable assets. I really appreciate his best effort to provide me a good platform for doing research. He is more than a thesis supervisor to me.

It has been also a great experience to work with Associate Professor Ho Weng Khuen from the National University of Singapore. He is more like my thesis cosupervisor. I am always impressed by his expertise on pointing out the issues and on the way to solve research problems. In addition, I would like to thank Prof. Jan M. Maciejowski from University of Cambridge for his valuable suggestions in my research direction.

All my friends at Nanyang Technological University made my study and life to be wonderful and memorable. In particular, I am very thankful to Zhou Dexiang, Sun Lu and Chen Xuebing, Dr. Eddy Foo, Ashok, who helped me to understand so many critical problems. I am thankful to Dr. Tri Tran for the discussions on some conference papers and report. I would like to thank my friends, Yang Cangjie, Wang Chenyi, Chen Rong, Liu Zhichao, Yang Rong, Yin Le, Kou Fei, Hung, Thuy 
and so on, for the pleasant time with them.

The last but not the least, I owe the most to my family and my wife, Lin Jing. I could never have achieved this goal without their generous love and support. 


\section{Abstract}

Power system state estimation (PSSE) plays an important role in power system operation. The Gaussian noise assumption is commonly made in PSSE. However, this assumption is only an approximation to reality. Outliers that are far away from the expected Gaussian distribution function can give rise to erroneous estimation results. Robust estimators such as Quadratic-Constant (QC), Quadratic-Linear (QL), Square-Root (SR), Multiple-Segment (MS) and Schweppe-Huber GeneralizedM (SHGM) have been introduced in the literature to solve the outlier problem in power systems. In this thesis, an analytical equation is derived using the Influence Function (IF), a tool from robust statistics, to calculate approximately the variances of the estimates of these robust estimators. This variance formula has many advantages: (i) It can be used to express the variance of state estimate as a function of measurement variances thus enabling the selection of sensors for specified estimator precision; (ii) It can be used to design an optimal estimator; (iii) Although numerical methods can also be used to find variance, the derived equation as a mathematical function is more insightful and requires less computational effort.

For robust PSSE, this thesis proposes a robust estimator based on the maximum likelihood criterion, and a noise model with $t$-distribution probability density function (pdf). The thick tail property of $t$-distribution down weights outliers so that the proposed estimator is robust to outliers. Instead of solving the optimization problem numerically, the IF is employed to give an approximate solution to reduce 
computational load.

In addition, a robust estimator based on the moving horizon estimation (MHE) technique is proposed for PSSE. This robust estimator is called re-weighted MHE. The proposed estimator reduces its sensitivity to the outliers by updating their error covariances in real time and then uses these re-weighted error covariances for robust PSSE. Compared with other robust state estimators such as MS and Least Absolute Value (LAV) estimator, one advantage of the proposed estimator is that it can directly incorporate constraints on the states to mitigate the outliers. If Phasor Measurement Units (PMUs) are used, the measurement model becomes linear. Then the proposed estimator can be formulated as a quadratic programming (QP) and solved by Alternating Direction Method of Multipliers (ADMM) algorithm efficiently. When the measurement model is nonlinear, the iterated RMHE (iRMHE) algorithm is proposed.

Finally, the centralized estimator is not applicable when the size of power system becomes very large. Two distributed versions of the proposed robust estimator based on MHE are considered: distributed MHE (DMHE) and partitioned MHE (PMHE). For DMHE, each local area will obtain the states of the whole system. It is suitable for the advanced applications such as wide-area monitoring systems (WAMSs) that require the system-wide state to be available to all the regional transmission organizations (RTOs). For PMHE, each local area only uses its local measurements and the states of border buses exchanged from its neighborhoods. It solves a smaller optimization problem to obtain the states of local states. Therefore, the communication load and computational load are reduced. 


\section{Contents}

Acknowledgements $\quad$ i

Abstract

List of Contents $\quad$ v

List of Figures $\quad$ x

List of Tables $\quad$ XV

Symbols and Acronyms xvii

1 Introduction 1

1.1 Motivation .......................... 1

1.2 Major Contributions of the Thesis . . . . . . . . . . . . . . . 4

1.3 Outline of the Thesis . . . . . . . . . . . . . . 7

2 Literature Review $\quad 8$

2.1 Robust State Estimation . . . . . . . . . . . . . . . . . . 8

2.2 Centralized and Distributed State Estimation Algorithms . . . . . . . 11

2.2.1 Centralized Algorithms . . . . . . . . . . . . . . . . 11

2.2.2 Distributed Algorithms . . . . . . . . . . . . . . . . . . 14 


\section{Variance Analysis of Robust State Estimation in Power Systems} using Influence Function ${ }^{1} \quad 19$

3.1 Measurement Models for PSSE . . . . . . . . . . . . . . . . 20

3.1.1 With Phasor Measurement Units . . . . . . . . . . . . . 20

3.1.2 Without Phasor Measurement Units . . . . . . . . . . . 21

3.2 Existing Robust State Estimation Algorithms . . . . . . . . . . . 22

3.2.1 The Multiple-Segment Estimator . . . . . . . . . . . 25

3.2 .2 The Square-Root Estimator . . . . . . . . . . . . . 25

3.2.3 The Schweppe-Huber Generalized-M Estimator . . . . . . . . 26

3.2.4 Pseudo-Code . . . . . . . . . . . . . . . . . 27

3.3 Influence Function for Robust PSSE . . . . . . . . . . . . . . . . . . . 29

3.3.1 Influence Function Analysis . . . . . . . . . . . . . . . . . . 29

3.3.2 IF for Multiple-Segment Estimator . . . . . . . . . . . . 31

3.3.3 IF for Square-Root Estimator . . . . . . . . . . . . . . . 31

3.3.4 IF for Schweppe-Huber Generalized-M Estimator . . . . . . . 31

3.3.5 The Weighted-Least-Squares Connection . . . . . . . . . . 31

3.4 Simulation Results . . . . . . . . . . . . . . . . . . . . . . 32

3.4.1 Example 1: Four Measurements, Three States . . . . . . . . . 34

3.4.2 Example 2: The IEEE 14-bus System with PMU measurements 36

3.4.3 Example 3: Optimal Estimator Design . . . . . . . . . . 43

3.4.4 Example 4: The IEEE 30-bus System with PMU measurements 46

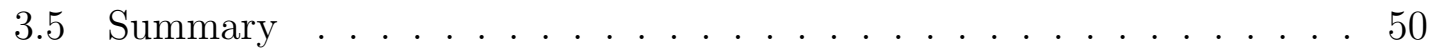

\section{Robust Power System State Estimation Using $t$-Distribution Noise} Model $^{2}$

${ }^{1}$ Chapter 3 is based on the paper "Weng Khuen Ho, Tengpeng Chen*(corresponding author), et al., Variance Analysis of Robust State Estimation in Power System using Influence Function, International Journal of Electrical Power \&3 Energy Systems, 92: 53-62 (2017)."

${ }^{2}$ Chapter 4 is based on our submitted paper "Tengpeng Chen, et al., Robust Power System State Estimation Using $t$-Distribution Noise Model. IEEE Transactions on Smart Grid, under 
4.1 Existing Robust State Estimation Algorithms . . . . . . . . . . . 52

4.2 The Proposed Robust Estimator . . . . . . . . . . . . . . . . 53

$4.2 .1 \quad t$ Distribution . . . . . . . . . . . . . . . 53

4.2.2 Maximum Likelihood Estimation . . . . . . . . . . . . 54

4.2.3 Influence Function Approximation . . . . . . . . . . . . . 55

4.2.4 The Weighted-Least-Squares Connection . . . . . . . . . . . . 57

4.3 Simulation and Experiment Results . . . . . . . . . . . . . . 58

4.3.1 Example 1: Simulations Based on Linear Measurement Model 58

4.3.2 Example 2: Experiment in NTU Microgrid . . . . . . . . . . 64

4.4 Summary . . . . . . . . . . . . . . . . . . . 67

5 Moving Horizon Estimation for Power Systems ${ }^{3} \quad 68$

5.1 Measurement Model and State Equation . . . . . . . . . . . . . 69

5.2 MHE for Power Systems with Linear Measurement Model . . . . . . . 70

5.2 .1 MHE ...................... 70

5.2 .2 Re-weighted MHE . . . . . . . . . . . . . . . . . . 74

5.3 MHE for Power Systems with Nonlinear Measurement Model . . . . . 76

5.4 Alternating Direction Method of Multipliers for MHE . . . . . . . . . 79

5.4.1 Alternating Direction Method of Multipliers . . . . . . . . . 80

5.4.2 Quadratic Programming Problem Formulation of MHE . . . . 81

5.4 .3 Standard ADMM Iterations . . . . . . . . . . . . . . . 82

5.4 .4 Over-relaxed ADMM Iterations . . . . . . . . . . . . . 83

5.5 Simulation Results . . . . . . . . . . . . . . . . . . 84

5.5.1 Simulations Based on Linear Measurement Model . . . . . . . 84

5.5.2 Simulations Based on Nonlinear Measurement Model . . . . . 93

review, 2017."

${ }^{3}$ Part of the materials in Chapter 5 is taken from paper "Tengpeng Chen. Robust State Estimation for Power Systems via Moving Horizon Strategy. Sustainable Energy, Grids and Networks, 10: 46-54 (2017)." 
5.6 Summary . . . . . . . . . . . . . . . . . . . . . . . . 97

6 Distributed Moving Horizon Estimation for Power Systems ${ }^{4} \quad 100$

6.1 Preliminaries . . . . . . . . . . . . . . . . 101

6.2 DMHE for Power Systems with Linear Measurement Model . . . . . . 101

6.3 DMHE for Power Systems with Nonlinear Measurement Model . . . . 106

6.4 Simulation Results . . . . . . . . . . . . . . . . . . . . . 110

6.4.1 Simulations Based on Linear Measurement Model . . . . . . . 110

6.4.2 Simulations Based on Nonlinear Measurement Model . . . . . 116

6.5 Summary . . . . . . . . . . . . . . . . . . . 121

7 Re-weighted Partitioned Moving Horizon Estimation for Power Systems $^{5}$

7.1 RPMHE for Power Systems with Linear Measurement Model . . . . . 123

7.1.1 RPMHE Problem Formulation . . . . . . . . . . . . . . . 123

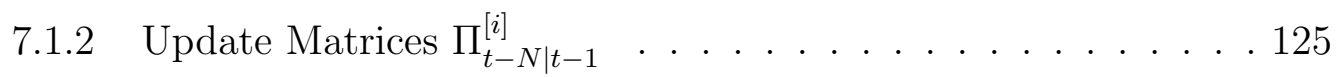

7.1.3 RPMHE Convergence Properties . . . . . . . . . . 126

7.2 Simulation Results . . . . . . . . . . . . . . . . . . . 129

7.2.1 Simulation on the IEEE 14 -bus system . . . . . . . . . . . 129

7.2.2 Simulation on the IEEE 118-bus system . . . . . . . . . . 132

7.3 Summary . . . . . . . . . . . . . . . . . . . . 135

8 Conclusion and Future Research $\quad 136$

8.1 Conclusion . . . . . . . . . . . . . . . . . 136

8.2 Recommendation for Future Research . . . . . . . . . . . . . . . 137

\footnotetext{
${ }^{4}$ Part of the materials in Chapter 6 is taken from paper "Tengpeng Chen, et al., Distributed Moving Horizon Estimation for Power Systems. IEEE Power 83 Energy Society General Meeting (PESGM), 1-5 (2015)."

${ }^{5}$ Part of the materials in Chapter 7 is taken from paper "Tengpeng Chen, et al., Application of Partitioned-Based Moving Horizon Estimation in Power System State Estimation. Power and Energy Engineering Conference (APPEEC), IEEE PES Asia-Pacific, 1-5 (2015)."
} 
Author's Publications

Bibliography

Appendix

A Weighted Least Squares Algorithm

B Influence Function

C WLS with Largest Normalized Residuals 


\section{List of Figures}

1.1 The diagram of $\operatorname{PSSE}[1] . \ldots \ldots \ldots \ldots$

1.2 The architecture of centralized state estimation approach. . . . . . . . 3

1.3 The architecture of distributed state estimation approach. . . . . . . 3

2.1 Scheme of hierarchical architeture $[2] \ldots \ldots \ldots \ldots$

2.2 Parallel distributed architecture illustrating 2 different communication schemes. . . . . . . . . . . . . . . 16

$3.1 \rho\left(e_{i}\right)$ of the Multiple-Segment (MS with $a_{i}=3, b_{i}=4, r_{i}=5$ ), Quadratic-Constant (QC with $a_{i}=3$ ), Quadratic-Linear (QL with $a_{i}=3$ ), Square-Root (SR with $a_{i}=3$ ) and Schweppe-Huber GeneralizedM (SHGM with $\left.a_{i}=3, \kappa_{i}=0.7\right)$ estimator. . . . . . . . . . . 27

3.2 The derivative of $\rho\left(e_{i}\right)$ for the Multiple-Segment (MS with $a_{i}=3, b_{i}=$ $4, r_{i}=5$ ), Quadratic-Constant (QC with $a_{i}=3$ ), Quadratic-Linear (QL with $a_{i}=3$ ), Square-Root (SR with $a_{i}=3$ ) and Schweppe-Huber Generalized-M (SHGM with $\left.a_{i}=3, \kappa_{i}=0.7\right)$ estimator. . . . . . . 28

3.3 A simple example for PSSE with voltage measurements $V_{1}, V_{2}$, real and reactive power flow measurements $P_{21}, Q_{21}[3]$. . . . . . . 35

3.4 The IEEE 14-bus system installed with PMUs. . . . . . . . . . . . . . 37 
3.5 Variances of all the 28 state estimates versus $\sigma_{3}$. The solid-line is the variance of state estimate $\hat{V}_{12}^{r}$. The crosses are the variances of state estimate $\hat{V}_{12}^{r}$ obtained from simulation. . . . . . . . . . . . . . 42

3.6 Variance of state estimate $\hat{V}_{12}^{r}$ versus $\sigma_{i}, i=1, \ldots, 58$. The solid-line shows the variance of state estimate $\hat{V}_{12}^{r}$ versus $\sigma_{3}$. The crosses are the variances of state estimate $\hat{V}_{12}^{r}$ versus $\sigma_{3}$ obtained from simulation. 42

3.7 IF of maximum likelihood estimator: dash-dotted-line; MS (optimal) estimator: solid-line; MS (typical) estimator: dash-line. . . . . . . . . 46

3.8 The IEEE 30-bus system installed with PMUs [4] . . . . . . . . . . . 47

4.1 The $t$ distribution with different shape parameters. . . . . . . . . . . 54

4.2 The configuration of the real time simulation. . . . . . . . . . 60

4.3 The $t$ distribution with $\xi_{i}=4, \sigma_{i}^{t}=0.0065$ and Gaussian distribution with $\sigma_{i}=0.005$ and $\sigma_{i}=0.007$ are used to fit the noise generalized according to $(4.13)$ with $\sigma_{i}=0.005 \ldots \ldots \ldots$. . . . . . . 61

4.4 Microgrid with 1 programmable source and 1 load. . . . . . . . . 65

4.5 Equipments used in the microgrid. . . . . . . . . . . . . . 65

4.6 The $t$ distribution with $\xi_{i}=3, \sigma_{i}^{t}=4 \times 10^{-5}$ and Gaussian distribution with $\sigma_{i}=3.0 \times 10^{-5}$ and $\sigma_{i}=4.2 \times 10^{-5}$ are used to fit the raw measurement (the real power flow from Bus 3 to Bus 2) collected from microgrid. . . . . . . . . . . . . . . . . . . 66

4.7 The estimated result $\hat{V}_{2}^{r}$ when outliers occur in measurement (the real power flow from Bus 3 to Bus 2). . . . . . . . . 66

5.1 Moving horizon window. . . . . . . . . . . . . . . 72

5.2 Mean square errors of $\operatorname{WLS}(1), \operatorname{MS}(1)$ and $\operatorname{RMHE}\left(Q \rightarrow \infty, R_{k}^{q}=R\right)$ without constraints in the IEEE 14-bus system. . . . . . . . . . . . 87 
5.3 Mean square errors of WLS, MS and $\operatorname{RMHE}\left(Q=10^{-5} I, N+1=1\right)$ with constraints in the IEEE 14 -bus system. . . . . . . . . . . . . . . 89

5.4 Mean square errors of WLS, MS and $\operatorname{RMHE}\left(Q=10^{-6} I, N+1=3\right)$ with constraints in the IEEE 14-bus system. . . . . . . . . . . . 89

5.5 Mean square errors of WLS, MS and $\operatorname{RMHE}\left(Q=10^{-6} I, N+1=3\right)$ with constraints in the IEEE 30-bus system. . . . . . . . . . . . . . . 90

5.6 Real component of Bus 2 voltage phasor estimated by different estimators in IEEE 14-bus system. . . . . . . . . . . . . . . . . . . . 91

$5.7\|\bar{r}\|^{2},\|\bar{s}\|^{2}$ (the solid curve) and $\epsilon^{\text {pri }}, \epsilon^{\text {dual }}$ (the dotted curve). . . . . . 92

5.8 The IEEE 14-bus system with SCADA measurements. . . . . . . . . 94

5.9 The IEEE 30-bus system with SCADA measurements. . . . . . . . . 95

5.10 Mean square errors of $\operatorname{WLS}(1)$ and $\operatorname{iRMHE}(Q \rightarrow \infty, N+1=1)$ without constraints in Example 3. . . . . . . . . . . . . . . . 98

5.11 Mean square errors of $\operatorname{WLS}(1)$ and iRMHE $\left(Q=10^{-5} I, N+1=3\right)$ with constraints in the IEEE 14-bus system. . . . . . . . . . . . . . 98

5.12 Mean square errors of $\operatorname{WLS}(1)$ and iRMHE $\left(Q=10^{-5} I, N+1=3\right)$ with constraints in the IEEE 30-bus system. . . . . . . . . . . . . . 99

6.1 The IEEE 14-bus system installed with PMUs is separated into 4 local areas. . . . . . . . . . . . . . . . . . . . 111

6.2 Communication scheme and matrix $K \ldots \ldots . \ldots 111$

6.3 The IEEE 30-bus system installed with PMUs is separated into 4 local areas . . . . . . . . . . . . . . . . . . . 112

6.4 MSE of WLS, MHE and DMHE with constraints in the IEEE 14-bus system. . . . . . . . . . . . . . . . . . . . . 114

6.5 MSE of WLS, MHE and DMHE with constraints in the IEEE 30-bus system. . . . . . . . . . . . . . . . . . . 114 
6.6 Real component of Bus 2 voltage phasor estimated by WLS, MHE and DMHE with constraints in the IEEE 14-bus system. . . . . . . . 115

6.7 Imaginary component of Bus 12 voltage phasor estimated by WLS, MHE and DMHE with constraints in the IEEE 14-bus system. . . . . 115

6.8 The IEEE 14-bus system with SCADA measurements. . . . . . . . . 117

6.9 Communication scheme and matrix $K \ldots \ldots . \ldots . \ldots . \ldots 17$

6.10 The IEEE 30-bus system with SCADA measurements. . . . . . . . . 118

6.11 MSE of WLS, iMHE and iDMHE with constraints in the IEEE 14-bus system. . . . . . . . . . . . . . . . . . . . 119

6.12 MSE of MHE, iMHE, DMHE and iDMHE with constraints in the IEEE 30-bus system. . . . . . . . . . . . . . . . . . . . . 119

6.13 Bus 2 estimated voltage by WLS, iMHE and iDMHE with constraints in the IEEE 14 -bus system. . . . . . . . . . . . . . . . 120

6.14 Bus 12 estimated phase angle by WLS, iMHE and iDMHE with constraints in the IEEE 14 -bus system. . . . . . . . . . . . . . . . . 120

7.1 The communication scheme related to the partitioned areas. . . . . . 129

7.2 MSE of WLS, MS, RMHE and RPMHE with constraints in the IEEE 14-bus system. . . . . . . . . . . . . . . . . . 131

7.3 Real part of Bus 2 voltage phasor estimated by RPMHE with constraints (after converging) in the IEEE 14-bus system. . . . . . . . . . 131

7.4 Imaginary part of Bus 14 voltage phasor estimated by RPMHE with constraints (after converging) in the IEEE 14-bus system. . . . . . . . 132

7.5 The IEEE 118-bus system with 6 partitioned local areas (subsystems)

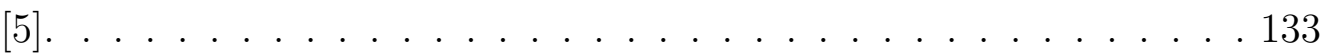

7.6 The communication scheme related to the partitioned IEEE 118-bus system. . . . . . . . . . . . . . . . . . . 133 
7.7 MSE of WLS, SR, RMHE and RPMHE with constraints in the IEEE 118-bus system. . . . . . . . . . . . . . . . . 134 


\section{List of Tables}

3.1 Parameters of estimators and noises used in the IEEE 14-bus system. 33

3.2 Variances of the state estimates (WLS, QC, QL) in the IEEE 14-bus system using the linear measurement model in (3.1) . . . . . . . . . . 40

3.3 Variances of the state estimates (SR, MS, SHGM) in the IEEE 14-bus system using the linear measurement model in (3.1). . . . . . . . . . . 41

3.4 Variances of the state estimates of the MS (optimal) and MS (typical) estimators in the IEEE 14-bus system. . . . . . . . . . . . . . . . 45

3.5 Parameters of estimators and noises used in the IEEE 30-bus system. 48

3.6 Variances of the state estimates from the MS estimator for the IEEE 30-bus system with PMU measurements. . . . . . . . . . . . . . 49

4.1 Parameters of estimators and noises used in the simulation examples. $\quad 59$

4.2 Variances of the state estimates of the different estimators in the IEEE 14-bus system with PMU measurements. . . . . . . . . . . . . . 62

4.3 Variances of the state estimates of the different estimators in the IEEE 118-bus system with PMU measurements. . . . . . . . . . . 63

4.4 The computational time (per step) of different estimators in the IEEE 118-bus system. . . . . . . . . . . . . . . . . 64

5.1 The $\mathrm{AMSE}^{\natural}$ values for different estimators with different parameters in the simulation examples. . . . . . . . . . . . . 86 
5.2 The average computational time (per step) of different estimators based on linear measurement model. . . . . . . . . . . . . . . . . . . 93

5.3 The $\mathrm{AMSE}^{\natural}$ for different estimators with different parameters in the simulation examples. . . . . . . . . . . . . . . . . . . . 96

5.4 The computation time (per step) of different estimators based on nonlinear measurement model . . . . . . . . . . . . . . . . . . 97

6.1 Measurements (ignoring the subscript "mea") allocated for each area. 112

6.2 The AMSE and computation time (per step) of MHE and DMHE with constraints based on linear measurement model. . . . . . . . . 116

6.3 The AMSE and computation time of the iDMHE based on nonlinear measurement model. . . . . . . . . . . . . . . . . . . 118

7.1 The AMSE and computation time (per step) of RMHE and RPMHE with constraints in the IEEE 14-bus system. . . . . . . . . . . . . 130

7.2 The comparison of AMSE and computation time (per step) of the RMHE and RPMHE with constraints in the IEEE 118-bus system. . 134 


\section{Symbols and Acronyms}

\section{Symbols}

$m$

$m_{i}$

$n$

$n_{i}$

$x$

$x^{[i]}$

$\hat{x}$

$\bar{x}_{0}$

$\hat{x}_{t}$

$\hat{x}_{t}^{q}$
Number of measurements at time step $k$

Number of local measurements at area $i$ at time step $k$

Number of the system-wide states

Number of local states at area $i$

True state vector, $x \in \mathbb{R}^{n}$

True local states in area $i, x^{[i]} \in \mathbb{R}^{n_{i}}$

The estimated state vector when the time step is from 1 to $N$,

$\hat{x}_{1}=\ldots=\hat{x}_{N}=\hat{x}$

The initial estimate of $x_{0}$

State vector estimated by the MHE at time step $t$

State vector estimated by the MHE at iteration index $q$ within time step $t$

$\hat{x}_{i, t}$

State vector estimated by local area $i$ within time step $t$ 


\begin{tabular}{|c|c|}
\hline$\left(\hat{x}_{t}^{[i]}\right)^{q}$ & $\begin{array}{l}\text { Local state vector estimated by the PMHE at iteration index } \\
q \text { within time step } t\end{array}$ \\
\hline$z$ & Measurements from Phasor Measurement Units (PMUs) \\
\hline$\breve{z}_{j_{1}, k}$ & Local measurements in area $j_{1}$ within time step $k$ \\
\hline$z_{k}^{[i]}$ & Local measurements in area $i$ within time step $k$ \\
\hline$y$ & Measurements from the SCADA system \\
\hline$P_{i j}$ & Real power flow from bus $i$ to bus $j$ \\
\hline$Q_{i j}$ & Reactive power flow from bus $i$ to bus $j$ \\
\hline$\epsilon_{k}\left(\right.$ or $\left.v_{k}\right)$ & Vector of measurement noise at time step $k$ \\
\hline$w_{k}$ & Vector of process noise at time step $k$ \\
\hline$w_{k}^{[i]}$ & $\begin{array}{l}\text { Process noise at local area } i \text { in the PMHE algorithm, } w^{[i]_{k}} \in \\
\mathbb{R}^{n_{i}}\end{array}$ \\
\hline$f_{i}\left(\epsilon_{i, k}\right)$ & $\begin{array}{l}\text { The probability density function (pdf) of the } i \text { th measurement } \\
\text { noise }\end{array}$ \\
\hline$\epsilon_{i, k}$ & $\begin{array}{l}\text { A zero-mean independent random variable associated with pdf } \\
f_{i}\left(\epsilon_{i, k}\right) \text {. }\end{array}$ \\
\hline$\epsilon_{k}^{T}$ & Transpose of noise vector $\epsilon_{k}$ \\
\hline$\varepsilon$ & Vector of measurement noise over a time interval, $\left[\epsilon_{1}^{T}, \ldots, \epsilon_{N}^{T}\right]^{T}$ \\
\hline$W_{i}$ & Weighting factor for measurement $i$ \\
\hline$V_{i}$ & Magnitude of the voltage at bus $i$ \\
\hline$\delta_{i}$ & Phase angle at bus $i$ \\
\hline
\end{tabular}


$V_{i}^{r}$

$V_{i}^{i m}$

$\{\cdot\}^{\text {mea }}$

$\left\{I_{i j}^{r}\right\}^{\text {mea }}$

$\left\{I_{i j}^{i m}\right\}^{m e a}$

$B_{i i}$

$G_{i j}+j B_{i j}$

$e_{i}$

$e_{i, k}^{n o r m}$

$\rho\left(e_{i}\right)$

$J\left(\right.$ or $\left.\left.J_{o}\right)\right)$

$\Psi\left(\right.$ or $\left.\left.\Psi_{o}\right)\right)$

$h_{i}(x)$

$H$

$H_{k}^{q}$

$H_{i}$

$\breve{H}_{i}$ $i$ linear case $\breve{H}_{i} \in \mathbb{R}^{m_{i} \times n}$
The real part of the voltage phasor at bus $i$

The imaginary part of the voltage phasor at bus $i$

Measured value of the voltage phasor or line current

The real part of the current measurement $\left\{I_{i j}\right\}^{\text {mea }}$

The imaginary part of the current measurement $\left\{I_{i j}\right\}^{\text {mea }}$

The shunt admittance at bus $i$.

The series admittance of line connecting bus $i$ and bus $j$,

Measurement residual $i$

The normalized measurement residual $i$ at time step $k$

A nonlinear function of $e_{i}$

Total cost function

Derivative of $J\left(\right.$ or $\left.\left.J_{o}\right)\right)$ wrt $\hat{x}$

Nonlinear function relating the state vector $x$ to measurement

Measurement matrix in linear case

The Jacobian matrix between measurements and states in non-

The $i$-th row of matrix $H$

Measurement matrix at local area $i$ in the DMHE algorithm, 


\begin{tabular}{|c|c|}
\hline$H^{[i]}$ & $\begin{array}{l}\text { Measurement matrix at local area } i \text { in the PMHE algorithm, } \\
H^{[i]} \in \mathbb{R}^{m_{i} \times n_{i}}\end{array}$ \\
\hline$H^{T}$ & Transpose of matrix $H$ \\
\hline $\mathbf{I F}(\cdot)$ & Influence function \\
\hline $\operatorname{erf}(\cdot)$ & Gauss error function \\
\hline$\Gamma(\cdot)$ & Gamma function \\
\hline$N$ & Number of sets of measurements \\
\hline$N m$ & Total measurement numbers for estimation \\
\hline$i$ & Measurement index \\
\hline$k($ or $t)$ & Time step \\
\hline$t_{c}$ & The converging time step \\
\hline$t-N \mid t-N-1$ & The time step for prediction from step $t-N-1$ to $t-N$ \\
\hline$\hat{x}_{t-N \mid t-N-1}$ & The prior MHE estimation at time step $t-N$ \\
\hline$q$ & Iteration index \\
\hline$q_{\max }$ & The iteration maximum number \\
\hline$Q$ & Covariance matrix of process noise \\
\hline$R$ & Covariance matrix of measurement noise \\
\hline$P$ & State covariance matrix \\
\hline$\Pi_{i, t-N \mid t-1}$ & The consensus weights for local area $i$ at $t-N \mid t-1$ \\
\hline$S$ & A positive definite matrix \\
\hline
\end{tabular}


$\Delta$

$K$

$\mathscr{O}_{N}^{[i]}$

$a_{i}$

$b_{i}$

$r_{i}$

$\kappa_{i}$

$\sigma_{i}$

$\Omega, \Lambda$

$\xi_{i}, \sigma_{i}^{t}$

$\bar{x}$

$\Theta^{*}$

$\Theta_{i}^{*}$

$\Psi$

$\Phi_{t-N}$

$\Phi_{i, t-N}$

$\ell$

$\delta_{R M H E}$

$\delta_{D M H E}$

\section{Estimation error}

A matrix link to the communication scheme

Local observability matrix at area $i$

Estimator first threshold parameter for $e_{i}$

Estimator second threshold parameter for $e_{i}$

Estimator third threshold parameter for $e_{i}$

The penalty factor in SHGM

Standard deviation of noise $\epsilon_{i}$

Diagonal matrix

The $t$-distribution parameters (shape parameter and scale parameter)

Operating point

Cost function for the MHE

Cost function for the DMHE at local area $i$

Chosen function for the MHE

The arrival cost (or called "initial penalty") at time step $t-N$

The arrival cost for local area $i$ at time step $t-N$

The partitioned number of local areas

The iterative threshold for the RMHE

The iterative threshold for the DMHE 
$\delta_{i R M H E}$

$\delta_{i D M H E}$

$\delta_{R P M H E}$

$\delta_{W L S}$

$\bar{f}, \bar{g}$

$\tau$

$I_{+}(\cdot)$

$\rho_{0}$

$\phi$

$L_{\rho_{0}}$

$\alpha$

$C, B$

$\mathbf{x}, Z$

$(k)$

$\rho_{0}$

$\bar{r}^{(k+1)}$

$\bar{s}^{(k+1)}$

$G_{m}$

$\bar{\Omega}$

$A^{-1}$
The iterative threshold for the iRMHE

The iterative threshold for the iDMHE

The iterative threshold for the RPMHE

The iterative threshold for the WLS

Convex function in the ADMM

The slack variables

The indicator function of $\tau$

The penalty parameter

Lagrange multipliers

The augmented Lagrangian for the ADMM

The relaxation parameter

Matrices used in ADMM

Vectors used in the ADMM

Iteration index in the ADMM

The penalty parameter in the ADMM

The primal residuals in the ADMM

The dual residuals in the ADMM

The empirical distribution of the observations

The residual covariance matrix

Inverse of matrix $A$ 
$\operatorname{diag}\left(A_{1}, \ldots, A_{n}\right) \quad$ Block diagonal matrix

$A \otimes B \quad$ Kronecker product of $A$ and $B$

$\|\cdot\| \quad \ell^{2}$ norm for vector or the induced norm for matrix

$\mathbb{R} \quad$ Set of real numbers

$\mathbb{Z} \quad$ Set of integers

$\mathbb{X} \quad$ Constraint set for state $x$

$\mathbb{K} \quad$ Constraint set for $\mathbf{x}$

0 Zero vector with a compatible dimension

$1 \quad$ One Vector with a compatible dimension

$I_{n} \quad$ Identity matrix with dimension of $n \times n$

$\mathbf{0}_{\mathbf{m} \times \mathbf{n}} \quad$ Zero matrix with dimension of $m \times n$

$N(\sigma) \quad$ Gaussian distribution with 0 mean and standard deviation $\sigma$

$\operatorname{Var}(\cdot) \quad$ Variance operation

$\mathcal{N}_{i} \quad$ Neighbors of area $i$

\section{Acronyms}

PSSE Power system state estimation

RTUs Remote terminal units

WLS Weighted least squares

IRWLS Iteratively re-weighted least squares 
LAN Local area network

EMS Energy management system

LNR Largest normalized residuals

SCADA Supervisory control and data acquisition

MPC Model predictive control

MHE Moving horizon estimation

RMHE Re-weighted moving horizon estimation

iRMHE Iterated re-weighted moving horizon estimation

iMHE Iterated moving horizon estimation

DMHE Distributed moving horizon estimation

iDMHE Iterated distributed moving horizon estimation

PMHE Partitioned moving horizon estimation

RPMHE Re-weighted partitioned moving horizon estimation

BDS Bad data suppression

pdf Probability density function

wrt With respect to

LP Linear programming

PMUs Phasor measurement units

IF Influence function

MS Multiple-Segment 
QC Quadratic-Constant

QL Quadratic-Linear

SR

Square-Root

SHGM Schweppe-Huber Generalized-M

LAV Least absolute value

WLAV Weighted least absolute value

MSE Mean square error

AMSE Average of mean square error

QP Quadratic programming

MLE Maximum likelihood estimation

KF Kalman filter

ADMM Alternating direction method of multipliers

WAMSs Wide-area monitoring systems

RTOs Regional transmission organizations

PS Projection statistics

SV Sum of the state estimate variances

ML Maximum likelihood

LS Least squares

i.i.d Independent and identically distributed 


\section{Chapter 1}

\section{Introduction}

\subsection{Motivation}

In order to reduce the influence from the measurement noise and get an accurate estimate of the states of power systems, power system state estimation (PSSE) has been widely implemented [6-10]. Fig. 1.1 shows a block diagram of PSSE. Firstly the remote terminal units (RTUs) distributed in different parts of the power systems record measurement data including the bus voltage magnitudes, real and reactive power injections, real and reactive power flows and current magnitudes [6-8]. These RTUs are connected to a local area network (LAN) along with a supervisory control and data acquisition (SCADA) front end computer, which supports the communication of the collected measurements to the state estimator [6]. The RTUs can also record the status of the switching devices which is sent to the topology processor in the energy management system (EMS). According to the measurements and the topology status, the state estimator processes and then outputs the optimal estimated states consisting bus phase angles and voltage magnitudes. PSSE plays an important role in the power systems because the state estimation results, in real time, are usually used to monitor and control power systems for reliable operations 
$[6,11]$.

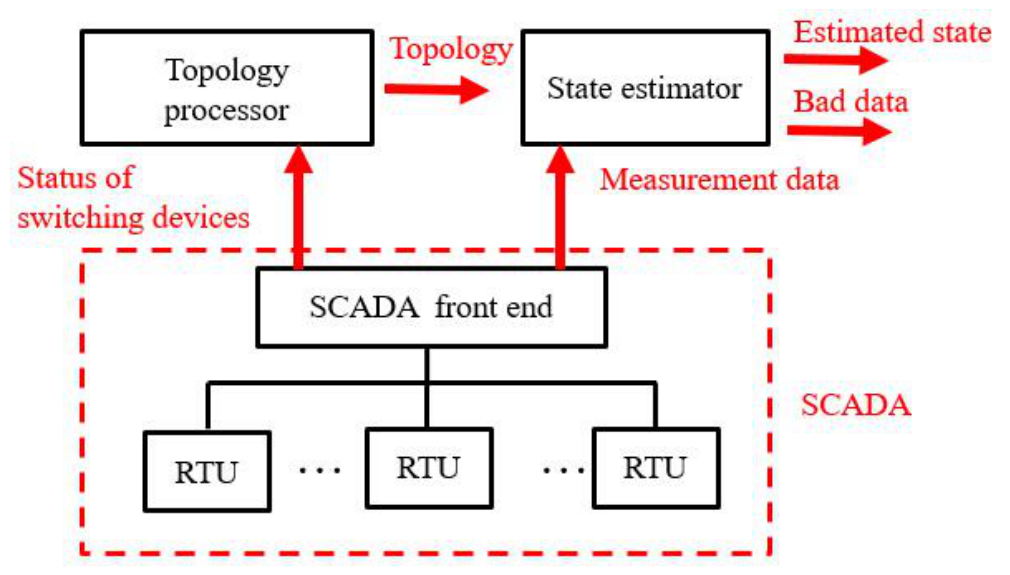

Figure 1.1: The diagram of PSSE [1].

The Gaussian noise assumption is commonly used in PSSE [6,12]. However, this assumption is only an approximation to reality. For example, transient data in steady-state measurement, instrument failure, human error or model nonlinearity can generate non-Gaussian measurement errors $[13,14]$. Outliers that are far away from the expected Gaussian distribution function can give rise to erroneous estimation results [15]. Robust estimators such as Quadratic-Constant (QC), Quadratic-Linear (QL), Square-Root (SR), Multiple-Segment (MS) and SchweppeHuber Generalized-M (SHGM) have been introduced in the literature to deal with outliers in power systems. Besides research papers, these robust estimators are also discussed in books $[6,7]$ and surveys [16-20]. However, variance analysis of these robust state estimators under non-Gaussian noise assumption is not presented.

Both centralized and distributed approaches for PSSE have been proposed, see Figs. 1.2 and 1.3 for illustration. For the centralized method, all measurements in local areas are sent to a centralized estimator. One centralized state estimation method named weighted least squares (WLS) is widely used for PSSE employing nonlinear measurement model $[6,7]$. However, there exists some disadvantages when using the centralized approach: (1) The communication load would be large when a 
large interconnected grid includes thousands of measurements recorded by the smart meters. All these measurements will be sent to some control center even though the distance is long; (2) The computational requirements of the centralized estimator may become formidable in order to process these measurements. A centralized estimator is practically infeasible due to the complexity scale of large grid.

Wide-area monitoring becomes popular in modern large-scale power systems. In order to reduce the computational complexity and communication load, various techniques on distributed state estimation have been proposed in [21-24]. For the distributed state estimation, the state estimators are installed at each local areas. They use their local and neighbors' measurements only, and share the estimated results with their neighbors. Most of approaches fall into the WLS category. These distributed algorithms can converge to the centralized results of the WLS. However, these distributed algorithms based on WLS do not take into account the physical constraints such as the upper and lower limits on states during the estimation process. The constrained moving horizon estimation (MHE) can be used to handle constraints. One key advantage of the constrained MHE is its ability to consider constraints (on states or noises) which prevent the estimator from giving the unreasonable estimates.

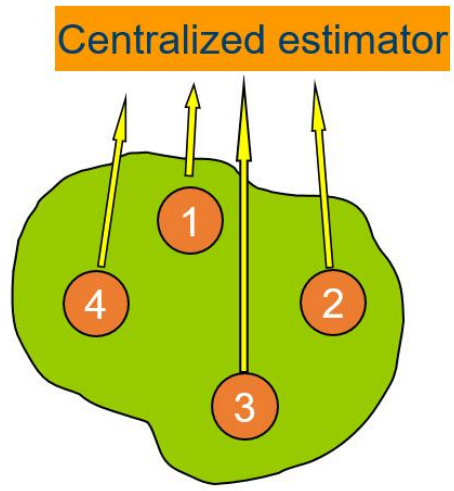

Figure 1.2: The architecture of centralized state estimation approach.

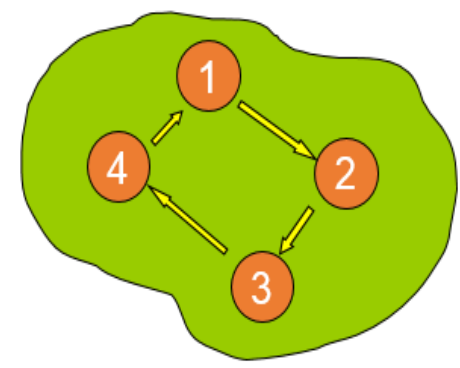

Figure 1.3: The architecture of distributed state estimation approach. 


\subsection{Major Contributions of the Thesis}

The main contributions of this dissertation are stated as follows:

1. Using influence function (IF) approximation, an analytical equation is derived to approximately calculate the variances of robust estimators such as $\mathrm{QC}, \mathrm{QL}$, SR, SHGM and MS. The IF has previously been used as an analytical tool in robust statistics $[25,26]$ and filter design with non-Gaussian noise assumption [13]. The derived analytical variance equation is useful. Firstly, it can be used to express the variance of a state estimate as a function of measurement variances enabling the selection of sensors for specified estimation precision. Secondly, it can be used to design an optimal estimator. Finally, although numerical methods can also be used to find the variance, the equation derived in this thesis as a mathematical function is more insightful than just a numerical answer. This is reported in Chapter 3 and is based on the following publication:

Weng Khuen Ho, Tengpeng Chen*(corresponding author), Keck Voon Ling, and Lu Sun. Variance Analysis of Robust State Estimation in Power System using Influence Function, International Journal of Electrical Power 85 Energy Systems, 92: 53-62 (2017).

2. A robust estimator is proposed, based on the maximum likelihood criterion for robust power system state estimation, where the $t$-distribution probability density function (pdf) is used to fit the measurement noise. Instead of solving the maximization problem numerically, the IF is employed to give an approximate solution, so that the proposed estimator can be implemented within the framework of the existing PSSE hardware and software. This is reported in Chapter 4 and is based on the following publication:

Tengpeng Chen, Lu Sun, Keck Voon Ling, and Weng Khuen Ho. "Robust 
Power System State Estimation Using $t$-Distribution Noise Model". IEEE Transactions on Smart Grid, under review, 2017.

3. The constrained moving horizon estimation (MHE) approach is adopted for PSSE. A re-weighted moving horizon estimation (RMHE) is proposed to improve the robustness for power systems. The states are estimated by minimizing an overall objective function which consists of sensor model error, process model error, and error in the state estimate at the beginning of the window [27]. The RMHE reduces its sensitivity to the outliers by updating their error covariances real-time and re-weighting their contributions adaptively for robust PSSE. Compared with the common robust state estimators such as the QC, QL, SR, MS, SHGM and LAV estimator, one advantage of the RMHE is that it takes the constraints on states into account during the optimization process to avoid suboptimal estimates [28]. In addition, the RMHE is extended to the nonlinear measurement model and the iterated RMHE (iRMHE) algorith$\mathrm{m}$ is proposed. This is reported in Chapter 5 and is based on the following publications:

Tengpeng Chen, Tri Tran, Dexiang Zhou, Weng Khuen Ho, and Keck Voon Ling. Iterated Moving Horizon Estimation for Power Systems. International Conference on Control, Automation and Information Sciences (ICCAIS), 81$86(2014)$

Tengpeng Chen. Robust State Estimation for Power Systems via Moving Horizon Strategy. Sustainable Energy, Grids and Networks, 10: 46-54 (2017).

4. In order to reduce the communication load, the distributed MHE is proposed. Under the distributed architecture, the power grid is separated into several non-overlapping local control areas. The measurements are not transmitted to a control center but to different local control areas which are able to communi- 
cate with its neighborhoods. Each local control area then estimates the states using only its own local measurements and measurements from its neighboring areas. Under the scheme of DMHE, each local area will obtain the states of the whole system. It is suitable for the applications such as wide-area monitoring and control that require the system-wide states to be available to all the regional transmission organizations (RTOs). This is reported in Chapter 6 and is based on the following publication:

Tengpeng Chen, Dexiang Zhou, Tri Tran, C. Kastner, K.-V Ling, K.-J. Tseng, and J.M. Maciejowski. Distributed Moving Horizon Estimation for Power Systems. IEEE Power \& Energy Society General Meeting (PESGM), 1-5 (2015). (One of the Best Conference Papers)

5. In order to reduce the communication load and computation load, a re-weighted partitioned MHE (RPMHE) method is proposed. Information exchanges between neighboring areas so the communication load is alleviated compared with the centralized method. Meanwhile, each local area solves a smaller optimization problem to estimate its own states so the computation load in each area becomes smaller. In order to reduce the influence of outliers further, the "re-weighted" process is considered. The RPMHE approach reduces its sensitivity to the outliers by re-weighting their error covariances online for robust PSSE. This is reported in Chapter 7 and is based on the following publication:

Tengpeng Chen, Ashok Krishnan, and Tri Tran. Application of PartitionedBased Moving Horizon Estimation in Power System State Estimation. Power and Energy Engineering Conference (APPEEC), IEEE PES Asia-Pacific, 1-5 (2015). 


\subsection{Outline of the Thesis}

The rest of the thesis is organized as follows:

Chapter 2 contains a literature review on robust PSSE, the centralized and distributed state estimation algorithms in PSSE is briefed.

In Chapter 3, an analytical equation is derived using influence function approximation to calculate the variance of the state estimate for the traditional robust state estimators such as the QC, QL, SR, SHGM and MS estimator.

In Chapter 4, a robust estimator based on the maximum likelihood criterion is proposed for robust PSSE, where the $t$-distribution probability density function (pdf) is used.

In Chapter 5, the re-weighted MHE (RMHE) is proposed. In addition, the implementation is extended to the nonlinear measurement model and the iterated RMHE (iRMHE) algorithm is proposed.

In Chapter 6, the DMHE algorithm is applied for linear measurement model and then the iterated DMHE (iDMHE) is proposed for the nonlinear case.

In Chapter 7, the re-weighted PMHE (RPMHE) method is proposed to reduce the computational load, the communication load and the influence of outliers.

In Chapter 8, the conclusions are drawn and some future research topics are discussed. 


\section{Chapter 2}

\section{Literature Review}

This chapter contains a review, focusing on the techniques of robust state estimation, as well as the centralized and distributed state estimation algorithms.

\subsection{Robust State Estimation}

Power system state estimation (PSSE) provides the estimated states of a power system from a set of measurements [6]. The weighted least squares (WLS) is a representative method and is effective for PSSE $[6,7]$. In WLS, the measurement errors are commonly assumed to have a Gaussian (Normal) distribution and the parameters for such a distribution are its mean, and its variance $\left(\sigma^{2}\right)$. The weights in WLS is inversely related to the assumed error variance for that measurement, $\sigma^{-2}[6]$. As a convenient reference, the WLS algorithm are given in Appendix A. It is well-known that WLS is not a robust estimator and the estimated states could have large errors when bad data occurs. Many researchers continuously broaden the applicability of WLS algorithm. For instance, the influence of the weighting matrix is investigated [29], where the weights are calculated by considering both the uncertainties associated with the measurement devices and the instrument transformers. This method can improve considerably the accuracy of state estimator [29]. 
Robust estimators have been developed to handle the outliers. The authors in [30] discuss a bad data suppression (BDS) method which is based on a non-quadratic cost function to suppress the bad measurements. This estimator reduces to the WLS estimator in the absence of bad data. Moreover, this approach inspires several robust estimators such as the Quadratic-Constant (QC), Quadratic-Linear (QL), SquareRoot (SR), Multiple-Segment (MS) and Schweppe-Huber Generalized-M (SHGM) estimator $[6,7,16-20,31,32]$. Each estimator has its own objective function which is in general a nonlinear function of the measurement residuals. The objective function is further modified to balance the influence of measurements and some threshold parameters need to be fixed in the objective function. Two methods based on the Newton's method and on iteratively re-weighted least squares method, can be used to solve the robust state estimation problem. The first method based on the Newton's method requires computation of the first and second derivatives of the objective function. The second method based on the iteratively re-weighted least squares algorithm avoids the computation of the second derivatives [6]. In recent years, another robust estimator, Least Absolute Value (LAV), is proposed in [4,33] where all measurements are assumed to be collected from PMUs. The LAV estimator is likely to populate in the future smart grid state estimation. It remains robust against bad data due to its automatic bad data rejection property [33]. The implementation of LAV can be made computationally efficient by taking advantage of power system properties and transforming the optimization problem into a Linear Programming (LP) problem then solve it efficiently using mature mathematical tool, e.g., the GUROBI [34]. In fact the LAV estimator is a special case of SHGM estimator when the threshold parameter is small [6].

Meliopoulos [3] proposes a variance equation for the WLS based on Gaussian noise assumption. The authors in [35] proposed a method to calculate the variances of estimators such as the WLS, weighted LAV (WLAV) and SHGM estimator. They 
assumed that all Gaussian noises have the same probability density function (pdf) and the threshold parameters for all measurement residuals are also the same. The same assumption can also be found in [36], where the influence function (see Appendix B) is used to calculate the error covariance matrices of the predictions and the state estimates. The asymptotic covariance matrix calculated by the influence function is used to avoid a degraded performance of the robust estimator [36]. However, the assumption that all measurement noises have the same pdf and threshold parameters may not be true in practice due to the large number of different smart meters and PMUs involved. There is a need to extend the variance analysis of robust state estimation to handle measurement noises with different distributions. Moreover, the Gaussian noise assumption is only an approximation to reality [13]. When the system meets outliers, instrument failure, human error or model nonlinearity $[13,14]$, non-Gaussian measurement error could also be generated. Outliers that are far away from the expected measuring data could give rise to misleading estimated results [15]. With the non-Gaussian noise assumption, the traditional way to obtain the variances of robust state estimation is to use the Monte Carlo method but this is very time consuming. Moreover, the current methods of choosing the parameters of the robust estimators are rather ad hoc, are based on the assumption of Gaussian noise. When measurement noise is not Gaussian, the optimal parameters for robust estimators will be changed. An analytical variance equation using IF is derived to solve these problems. See Chapter 3 for our contributions to this topic.

Considering that the Gaussian noise is only an approximation to reality, it is reasonable to find a better noise distribution to model the measurement noise in PSSE. The $t$-distribution has been employed in many applications from industrial manufacturing to statistical finance $[37,38]$. The $t$-distribution has the property of thick tail to better model the occurrence of outliers [39]. It has the flexibility to represent the Gaussian noise or non-Gaussian noise. For example, the $t$-distribution reduces 
to Gaussian distribution when its shape parameter tends to infinity. The heavy tail property of $t$-distribution can be used to improve the robustness of estimator with regard to outliers. In $[40,41]$, the Bayesian method and the heavy tail property of $t$-distribution are used to reduce the weights but the probability density function of the $t$-distribution is not directly used in these estimators. The authors in [42] present a maximum likelihood least squares with an iterative solving method. The iteration steps are too many and the convergence speed is quite slow. Other estimators based on the existing maximum likelihood estimation (MLE) can also be found in $[43,44]$. However, the numerical methods are not compatible with the current PSSE hardware and software. When the probability density function of the $t$-distribution noise is used directly in the likelihood function of MLE, the optimization problem is nonlinear and the common way is to solve it numerically [45]. We propose a method which uses the IF to approximately solve MLE and it can be implemented in the existing PSSE hardware and software. See Chapter 4 for details.

\subsection{Centralized and Distributed State Estimation}

\section{Algorithms}

In this section both centralized and distributed state estimation algorithms for power systems will be reviewed.

\subsubsection{Centralized Algorithms}

Under the centralized architecture, all measurements are transmitted to a centralized state estimator where a system-wide state estimation problem is solved. The WLS is a representative method and many algorithms based on the WLS have been proposed. A fast decoupled state estimator with bad data processing is described in [46], where the algorithm-decoupled estimator and the model-decoupled 
estimator are proposed. For the algorithm-decoupled estimator, the gain matrix is decoupled into several matrices with smaller dimensions, the off-diagonal elements of the gain matrix are considered to be 0 . The Jacobian matrices need to be recalculated at each iteration. The estimated states are only approximate results. For the model-decoupled estimator, the gain matrices would still need to be decoupled, but the decoupled Jacobian matrix elements are constant and calculated considering all voltages flat. It is only suggested for systems with high ratio of the system reactance to the system resistance. The conventional estimation assumes that the network topology is correct and the network parameters are sufficiently accurate. However, a topology error may occur due to faults in cable connection [47]. The authors in $[7,47]$ develop a generalised state estimation method in which the topology and parameter information is taken into account in the measurement model. A topology processor will check the switch status and the parameter estimation is also applied in advance. This method can promote a greater applicability of the WLS approach. Zhang et al [48] present a recursive PMU tuning algorithm to estimate the variance of PMU errors. The historical measurements are used to tune the corresponding weights. This tuning algorithm can be incorporated into the WLS algorithm so as to improve the performance. The authors in [49] formulate an alternative decoupled WLS method which is only applicable when the measurements are collected from PMUs. The original WLS problem is decoupled into two decoupled WLS problems, and each of them half the size of the original problem. Therefore, the computation can be implemented on two processors in parallel. Li et al [24] propose an adaptive re-weighted state estimation method. Instead of using a fixed error covariance, the error covariance applied in [24] is updated in real-time according to the measurements and the estimated states. The influence of bad data can be mitigated. A similar scheme is also presented in [50,51]. However, there exists drawbacks in the framework of WLS algorithm. When there is no any power source 
or load connected to the buses, the power injections will be zero. Such zero power injections used as virtual measurements can lead to ill-conditioned gain matrix and the WLS algorithm may become divergent. Several methods have been proposed which made use of constraints to improve the estimation accuracy. One method named equality-constrained is proposed to improve the performances [52]. These virtual measurements can be modelled as equality constraints so that their large weights can be excluded. Clements and Davis [53] present a new formulation which combines inequality constraints in PSSE. The inequality constraints are used to formulate limits on the reactive power at generators. In [54] the inequality-constrained formulations are used to deal with the measurement uncertainty in PSSE. These constraint limits define the tolerances on the measurements. Irving [55] introduces a robust generalized state estimator which is able to detect and reject three types of gross errors (parameter errors, measurement errors, and topology errors) simultaneously. The "uncertainty range" is adopted and a set of uncertain inequality constraints is considered in the optimization process. The use of constraints allows the gross errors to be judged and rejected.

The algorithms described so far are mostly based on the configuration of WLS and only measurement model is considered. The moving horizon estimation (MHE) is a kind of optimized state estimation method, which minimizes a cost function consisted of the error between the true states and the state estimation results, the error between the measurement and the predicted output, and an arrival cost which summaries the past data [56-58]. The optimization is often augmented with additional equality or inequality constraints. Adding constraints can overcome the issues such as the suboptimal estimates or instability of the error dynamics [59]. By having these constraints in the optimization, the MHE is more robust to the measurement outliers. In the industry implementation constraints on the state variables or noises are often taken as a priori. The reasonable constraints will keep the 
estimation problem tractable while the solution may be far from the true state if these constraints are set incorrectly [60]. As a special case, MHE without constraints becomes a Kalman filter (KF) or an iterated Extended Kalman filter [59,61].

The price to pay is an increased complexity since the computational load will increase and the optimizing problem has to be solved before one new set of measurements comes [62]. In order to accelerate the performance of MHE in PSSE, the alternating direction method of multipliers (ADMM) is investigated. The ADMM is an effective approach that solves convex optimization problems by breaking them into smaller pieces [63]. Therefore the optimization problem can be solved efficiently. Surveys of ADMM can be found in [64,65]. Applications of this method include the areas of machine learning, image processing, regularized estimation and so on. The applications of ADMM to quadratic programming (QP) problem is presented in [66]. The ADMM can be used as a "toolbox" to solve the MHE problem [67] and implement an algorithm of practical use.

The unconstrained MHE algorithm for PSSE is firstly proposed in [68] and the arrival cost is neglected. One drawback of this algorithm in [68] is that it truncates the connections with the past information such as the previous estimation results. Another drawback is that the constraints are not taken into account during the optimizing process. The state estimation algorithms based on MHE for linear and nonlinear measurement models will be presented in Chapter 5 .

\subsubsection{Distributed Algorithms}

Even though the structure of these centralized algorithms is simple, the centralized state estimator needs to be computationally powerful to process a large amount of data when the scale of power networks becomes large. In addition, the data transmission load may be large under the centralized architecture when the distance between the remote terminal units (RTUs) and the control center is especially long. 
With the development of communication network, advanced control, and the power industry reformation, the distributed state estimation approaches on the wide-area power systems have attracted more attention than ever before. A good survey on multi-area PSSE can be found in [69]. The existing distributed algorithms are usually composed of the hierarchical algorithms and parallel distributed algorithms.

The hierarchical architecture is composed of subsystem level and the coordination level, as shown in Fig. 2.1. The system is separated into several subsystems and every subsystem has one local estimator which works independently. The local solutions are then coordinated at the coordinated level. The decomposition in the hierarchical architecture includes the overlapping strategy [70-72] and the nonoverlapping one $[21,73]$. To some extent these methods can reduce computational burden. For example, Gómez-Expósito et al [21] describe a multilevel framework that facilitates seamless integration of existing state estimators that are designed to function at different levels of modeling hierarchy. A multilevel computation and communication architecture is proposed. The target is to accomplish very largescale monitoring of interconnected power systems. Korres [73] develops a method in which the distributed state estimator is used to solve a multi-area state estimation problem. Each local area uses local measurements, exchanges border information at a coordination state estimator, and solves for its own state estimation. However, they still suffer from the communication bottleneck. The estimation scheme is vulnerable because every local estimator needs to communicate with the coordinated estimator.

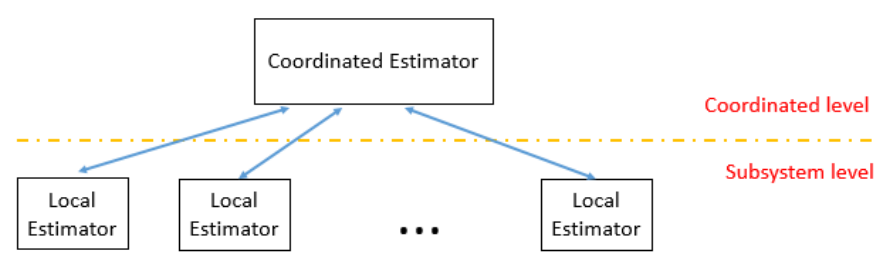

Figure 2.1: Scheme of hierarchical architeture [2]. 
Compared with the hierarchical architecture, the distributed architecture does not need a coordinated center and lets multiple parallel local control centers exchange local measurements (or estimation information) and perform global or state estimation. Therefore, the distributed architecture could have higher reliability. Moreover, the communication scheme is more flexible and the requirement on hardware is lesser due to its comparatively simple calculations. Under the distributed architecture, the global states are usually estimated by every local control center owing to the exchanging information only with its neighborhoods via the communication scheme, see a simple example in Fig. 2.2 where the system is separated into 4 local areas. The distributed algorithms can reduce the communication load. Many distributed algorithms are proposed in $[22-24,74,75]$, where each local estimator estimates the global state and finally converge to the centralized result. They are the distributed versions of the WLS estimator. It is important to note that the local observability assumption is not necessary in $[22,74]$. However, for each local area, the exchanging information is the system-wide estimated results. The communication load is still large when the scale of power network increases.

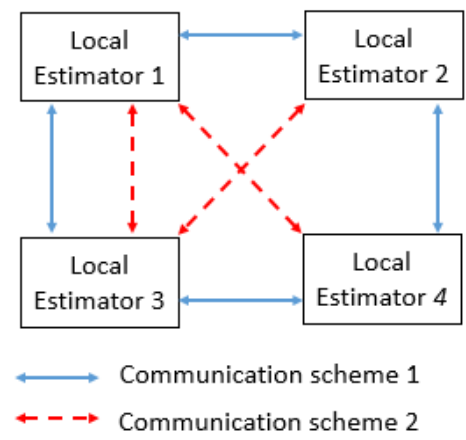

Figure 2.2: Parallel distributed architecture illustrating 2 different communication schemes.

As a summary, there exists a trade-off between the accuracy of state estimation results and the hardware requirement to choose which type of architecture. In addition, the physical constraints such as the lower and upper bounds on states are 
not exploited in the estimation process, which are usually taken as the known limits in the industry application.

\section{Distributed MHE}

Distributed MHE (DMHE) has been proposed in literature [76-78] for different applications. Farina et al [76] propose a distributed state estimation algorithm, DMHE, based on the concept of MHE. They prove convergence of the state estimates computed by any sensors to the correct state when constraints on noise and state variables are considered [76]. This approach provides a platform where the estimation problem can be solved in a distributed way. Each local control area will obtain the states of the whole system. It is suitable for the advanced applications such as wide-area monitoring and control that require the system-wide state to be available to all local control areas [79,80]. Haber [77] also presents a distributed MHE method for a large-scale systems that are described by sparse matrices. However, this paper is based on a specified system model. It is different from the models used in power systems so the method given in [77] can not be modified in PSSE. The applications of DMHE algorithms are extended to chemical reaction system$\mathrm{s}[78,81,82]$. The DMHE algorithm in [76] has been modified and its application in PSSE can be found in Chapter 6 .

\section{Partitioned MHE}

Under the scheme of DMHE introduced above, each local area estimates the states of the whole system. This is with high reliability because every local area can obtain the system-wide states. However, the computational load is still large. Schneider et al [83] propose a kind of partitioned-based moving horizon estimator. The estimated states in each local area are non-overlapping. However, it assumes an all-to-all communication network, that is, each local area needs to communicate 
to all other local areas. In this case the communication load would be quite large and it is not suitable to be implemented in the large-scale power systems. Farina et al [28] propose another distributed state estimation method, namely partitioned MHE (PMHE), for linear systems which is more reasonable and suitable for largescale systems monitoring. Now each local area solves for the local states via a smaller size optimizing problem and the computational load is small and scalable. Meanwhile, the information exchanges among local area estimators reduces to the states of border buses between neighboring areas, local measurements are not necessary to be exchanged anymore and communication load is alleviated. Applications of PMHE in PSSE will be presented in Chapter 7. 


\section{Chapter 3}

\section{Variance Analysis of Robust State}

\section{Estimation in Power Systems}

\section{using Influence Function ${ }^{1}$}

In this chapter an analytical equation is derived using influence function approximation to calculate the variance of the state estimate approximately for robust state estimators such as the QC, QL, SR, SHGM and MS estimator. The derived analytical variance equation is useful. Firstly, it can be used to express the variance of a state estimate as a function of measurement variances enabling the selection of sensors for specified estimation precision. Secondly, it can be used to design an optimal estimator. Finally, although numerical methods can also be used to find variance, the equation derived in this chapter as a mathematical function is more insightful than just a numerical answer.

\footnotetext{
${ }^{1}$ Chapter 3 is based on the paper "Weng Khuen Ho, Tengpeng Chen*(corresponding author), et al., Variance Analysis of Robust State Estimation in Power System using Influence Function, International Journal of Electrical Power 83 Energy Systems, 92: 53-62 (2017)."
} 


\subsection{Measurement Models for PSSE}

In this section the measurement models used for PSSE will be presented.

\subsubsection{With Phasor Measurement Units}

It is assumed that the system is observable by PMUs. With PMUs, measurements in voltages and currents can be expressed as linear functions of the system states (bus voltage phasors) [4,84]:

$$
\begin{aligned}
\left\{V_{i}\right\}^{\text {mea }} & =\left\{V_{i}^{r}\right\}^{\text {mea }}+j\left\{V_{i}^{i m}\right\}^{\text {mea }} \\
& =\left|V_{i}\right| \cos \left(\theta_{i}\right)+j\left|V_{i}\right| \sin \left(\theta_{i}\right) \\
\left\{I_{i j}\right\}^{\text {mea }} & =\left\{I_{i j}^{r}\right\}^{\text {mea }}+j\left\{I_{i j}^{i m}\right\}^{\text {mea }} \\
\left\{I_{i j}^{r}\right\}^{\text {mea }} & =G_{i j}\left(V_{i}^{r}-V_{j}^{r}\right)-B_{i j}\left(V_{i}^{i m}-V_{j}^{i m}\right)-B_{i i} V_{i}^{i m} \\
\left\{I_{i j}^{i m}\right\}^{\text {mea }} & =G_{i j}\left(V_{i}^{i m}-V_{j}^{i m}\right)+B_{i j}\left(V_{i}^{r}-V_{j}^{r}\right)+B_{i i} V_{i}^{r}
\end{aligned}
$$

where $\{\cdot\}^{\text {mea }}$ means that it is a measured value; $V_{i}^{r}$ and $V_{i}^{i m}$ are the real and imaginary parts of the voltage phasor of bus $i ; V_{i}$ and $\theta_{i}$ are the voltage magnitude and phase angle of bus $i ;\left\{I_{i j}\right\}^{\text {mea }}$ is the line current from bus $i$ to bus $j$; and $\left\{I_{i j}^{r}\right\}^{\text {mea }}$ and $\left\{I_{i j}^{i m}\right\}^{\text {mea }}$ are the real and imaginary parts of the current measurement $\left\{I_{i j}\right\}^{\text {mea }} . G_{i j}+j B_{i j}$ is the series admittance of line connecting bus $i$ and bus $j$, and $B_{i i}$ is the shunt admittance at bus $i$. The measurement vector $z \in \mathbb{R}^{m}$ includes $\left\{V_{i}^{r}\right\}^{m e a},\left\{V_{i}^{i m}\right\}^{m e a},\left\{I_{i j}^{r}\right\}^{m e a},\left\{I_{i j}^{i m}\right\}^{m e a}$ and the state vector is $x=\left[V_{1}^{r} V_{2}^{r} \cdots V_{n / 2}^{r} V_{1}^{i m} \cdots V_{n / 2}^{i m}\right]^{T} \in \mathbb{R}^{n}$. Therefore, the measurement equation can be written as:

$$
z_{k}=H x_{k}+\epsilon_{k}
$$


Chapter 3. Variance Analysis of Robust State Estimation in Power Systems using Influence Function ${ }^{1}$

where

$$
\begin{aligned}
z_{k} & =\left[z_{1, k}, z_{2, k}, \ldots, z_{m, k}\right]^{T} \\
x_{k} & =\left[x_{1, k}, x_{2, k}, \ldots, x_{n, k}\right]^{T} \\
\epsilon_{k} & =\left[\epsilon_{1, k}, \epsilon_{2, k}, \ldots, \epsilon_{m, k}\right]^{T}
\end{aligned}
$$

and $H$ is the measurement matrix. The $i$-th measurement taken at time step $k$ is defined as $z_{i, k}$, where $i=1, \ldots, m, k=1, \ldots, N . x_{k}$ means the system-wide states at time step $k$ and $x_{i, k}$ represents the $i$-th state at time step $k$. The $i$-th measurement noise at time $k, \epsilon_{i, k}$, is a zero-mean independent random variable associated with the probability density function (pdf) $f_{i}\left(\epsilon_{i, k}\right)$. A traditional power system may be considered as a quasi-static system $[85,86]$ because load demands change slowly and hence the states change slowly. The sampling time of PMU measurements is usually several milliseconds, while the estimates are usually updated once every few minutes if the measurements are collected from SCADA [8]. In this chapter it is assumed that the PMUs collect the measurements every 30 milliseconds [86] and the states are estimated for every $N$ sets of measurements, $k=1, \ldots, N$. Hence the estimated state can be taken as a constant vector within few minutes and it can be simply written as $\hat{x}_{1}=\cdots=\hat{x}_{N}=\hat{x}$.

\subsubsection{Without Phasor Measurement Units}

In a traditional power system where PMUs are not used, the traditional monitoring technologies implemented in the SCADA system can only take nonsynchronized measurements once every few seconds, for example, every five seconds or longer [86]. However, the estimates are usually updated once every few minutes in order to reduce the computational complexity required in implementing state estimation. The measurement $y_{k}$ containing the power injection and power flow is a nonlinear 
function of the state $x_{k}$ :

$$
y_{k}=h\left(x_{k}\right)+\epsilon_{k}
$$

where $x_{k}$ and $\epsilon_{k}$ are already defined in (3.1) and

$$
y_{k}=\left[\begin{array}{c}
y_{1}\left(x_{k}\right) \\
y_{2}\left(x_{k}\right) \\
\vdots \\
y_{m}\left(x_{k}\right)
\end{array}\right], h\left(x_{k}\right)=\left[\begin{array}{c}
h_{1}\left(x_{k}\right) \\
h_{2}\left(x_{k}\right) \\
\vdots \\
h_{m}\left(x_{k}\right)
\end{array}\right]
$$

The first-order Taylor series expansion about the operating point $\bar{x}$ is used to approximate the nonlinear function $h\left(x_{k}\right)$ in (3.2) to give

$$
\begin{gathered}
y_{k}=h(\bar{x})+H\left(x_{k}-\bar{x}\right)+\epsilon_{k} \\
H=\left.\frac{\partial h(x)}{\partial x}\right|_{x=\bar{x}}
\end{gathered}
$$

From (3.3) the measurement residual is given by

$$
e_{k}=y_{k}-h(\bar{x})+H \bar{x}-H x
$$

Let $z_{k}=y_{k}-h(\bar{x})+H \bar{x}$, then $(3.3)$ reduces to $(3.1)$.

\subsection{Existing Robust State Estimation Algorithms}

Robust PSSE has been discussed in the literature $[4,6,7,31-33,87,88]$. The authors in the cited references proposed their algorithms primarily on the single snap shot of measurements. However, the measurement outliers are removed by the robust 
Chapter 3. Variance Analysis of Robust State Estimation in Power Systems using Influence Function ${ }^{1}$

estimator when they are outside the MS estimator cut-off " $r_{i}$ " or the QC estimator cut-off " $a_{i}$ ", which will be presented later. Therefore, there could be insufficient data for the equations to solve for the estimates if only $N=1$ set of measurements is used. Given $N$ sets of $m$ measurements, the state estimate $\hat{x}$ can be obtained by minimizing the following objective function:

$$
J=\sum_{i=1}^{m} \sum_{k=1}^{N} \rho\left(e_{i, k}\right)
$$

where $\rho\left(e_{i, k}\right)$ is generally a nonlinear function. From (3.1), the measurement residual is given as

$$
e_{i, k}=z_{i, k}-H_{i} x
$$

The minimization of the cost function (3.5) using the Newton's method of iteration or the iteratively re-weighted least squares method is well documented in the literature [6]. The Newton's method approximates $J$ at each iteration by a quadratic function about the previous estimate of $x$. The iteratively re-weighted least squares algorithm avoids the second derivative required in the Newton's method. This chapter simply uses the iteratively re-weighted least squares algorithm given in [6].

Equation (3.6) gives $\frac{\partial e_{i, k}}{\partial x}=-H_{i}^{T}$. Differentiating the cost function (3.5) wrt $x$ gives

$$
\begin{aligned}
\frac{\partial J}{\partial x} & =\sum_{i=1}^{m} \sum_{k=1}^{N} \frac{\partial \rho\left(e_{i, k}\right)}{\partial e_{i, k}} \frac{1}{e_{i, k}} e_{i, k} \frac{\partial e_{i, k}}{\partial x} \\
& =-\sum_{i=1}^{m} \sum_{k=1}^{N} W_{i, k} e_{i, k} H_{i}^{T},
\end{aligned}
$$

where

$$
W_{i, k}=\frac{\partial \rho\left(e_{i, k}\right)}{\partial e_{i, k}} \frac{1}{e_{i, k}}
$$


Using (3.6), equation (3.7) can be written as

$$
\begin{aligned}
\frac{\partial J}{\partial x} & =-\sum_{i=1}^{m} \sum_{k=1}^{N} W_{i, k}\left(z_{i, k}-H_{i} x\right) H_{i}^{T} \\
& =-\bar{H}^{T} W(Z-\bar{H} x) \\
& =-\bar{H}^{T} W E \\
& \triangleq \Psi(E)
\end{aligned}
$$

where

$$
\begin{aligned}
\bar{H} & =\left[H^{T} \cdots H^{T}\right]^{T} \in \mathbb{R}^{N m \times n} \\
Z & =\left[z_{1}^{T} \cdots z_{N}^{T}\right]^{T} \in \mathbb{R}^{N m} \\
E & =\left[e_{1}^{T} \cdots e_{N}^{T}\right]^{T} \in \mathbb{R}^{N m} \\
W & =\operatorname{diag}\left(W_{1,1}, \ldots, W_{m, 1}, \ldots, W_{1, N}, \ldots, W_{m, N}\right) \in \mathbb{R}^{N m \times N m}
\end{aligned}
$$

Note that the superscript $(\cdot)^{T}$ represents the transpose operation. Because the system is observable, the matrix $\bar{H}^{T} W \bar{H}$ is full rank and invertible. Set $\Psi(E)=0$, then from (3.10) the estimation of $x$ is given by

$$
\hat{x}=\left(\bar{H}^{T} W \bar{H}\right)^{-1} \bar{H}^{T} W Z
$$

Using (3.4) and (3.8), the matrices $H$ and $W$ are updated and (3.12) is solved iteratively until the difference between the current and previous iteration for $\hat{x}$ is less than a specified tolerance [6]. The diagonal matrix $W$ for the MS, QC, QL, SR and SHGM estimator can be obtained as shown in Sections 3.2.1, 3.2.2 and 3.2.3. 
Chapter 3. Variance Analysis of Robust State Estimation in Power Systems using Influence Function ${ }^{1}$

\subsubsection{The Multiple-Segment Estimator}

The MS estimator is sometimes known as the Hampel estimator [26]. To obtain the matrix $W$ for the MS estimator, set

$$
\rho\left(e_{i, k}\right)= \begin{cases}\frac{\left(e_{i, k}\right)^{2}}{2 \sigma_{i}^{2}} & \left|e_{i, k}\right| \leq a_{i} \sigma_{i} \\ \frac{a_{i}\left|e_{i, k}\right|}{\sigma_{i}}-\frac{a_{i}^{2}}{2} & a_{i} \sigma_{i}<\left|e_{i, k}\right| \leq b_{i} \sigma_{i} \\ -\frac{a_{i}\left(r_{i} \sigma_{i}-\left|e_{i, k}\right|\right)^{2}}{2\left(r_{i}-b_{i}\right) \sigma_{i}^{2}}+\frac{1}{2} a_{i} r_{i}+\frac{1}{2} a_{i} b_{i}-\frac{1}{2} a_{i}^{2} & b_{i} \sigma_{i}<\left|e_{i, k}\right| \leq r_{i} \sigma_{i} \\ \frac{1}{2}\left(a_{i} r_{i}+a_{i} b_{i}-a_{i}^{2}\right) & r_{i} \sigma_{i}<\left|e_{i, k}\right|\end{cases}
$$

where $a_{i}, b_{i}$ and $r_{i}$ are thresholds selected by the user and $\sigma_{i}$ is the standard deviation of noise $\epsilon_{i, k}$.

Differentiating $\rho\left(e_{i, k}\right)$ wrt $e_{i, k}$ and substituting it into (3.8) gives

$$
W_{i, k}= \begin{cases}\frac{1}{\sigma_{i}^{2}} & \left|e_{i, k}\right| \leq a_{i} \sigma_{i} \\ \frac{a_{i}}{\sigma_{i}\left|e_{i, k}\right|} & a_{i} \sigma_{i}<\left|e_{i, k}\right| \leq b_{i} \sigma_{i} \\ \frac{a_{i}\left(r_{i} \sigma_{i}-\left|e_{i, k}\right|\right)}{\left(r_{i}-b_{i}\right) \sigma_{i}^{2}\left|e_{i, k}\right|} & b_{i} \sigma_{i}<\left|e_{i, k}\right| \leq r_{i} \sigma_{i} \\ 0 & r_{i} \sigma_{i}<\left|e_{i, k}\right|\end{cases}
$$

Note that $a_{i}<b_{i}<r_{i}$. The QC, QL and WLS estimators are the special cases of MS estimator. The MS estimator reduces to the QC estimator when $b_{i} \rightarrow a_{i}, r_{i} \rightarrow a_{i}$, the QL estimator when $b_{i} \rightarrow \infty$, and the WLS estimator when $a_{i} \rightarrow \infty[6]$.

\subsubsection{The Square-Root Estimator}

To obtain the matrix $W$ for the SR estimator, set

$$
\rho\left(e_{i, k}\right)= \begin{cases}\frac{\left(e_{i, k}\right)^{2}}{2 \sigma_{i}^{2}} & \left|e_{i, k}\right| \leq a_{i} \sigma_{i} \\ 2 a_{i}^{3 / 2} \sqrt{\frac{\left|e_{i, k}\right|}{\sigma_{i}}}-\frac{3}{2} a_{i}^{2} & \text { otherwise }\end{cases}
$$


Differentiating $\rho\left(e_{i, k}\right)$ wrt $e_{i, k}$ and substituting into (3.8) gives

$$
W_{i, k}= \begin{cases}\frac{1}{\sigma_{i}^{2}} & \left|e_{i, k}\right| \leq a_{i} \sigma_{i} \\ \sqrt{\frac{a_{i}^{3}}{\sigma_{i}\left|e_{i, k}\right|^{3}}} & \text { otherwise }\end{cases}
$$

\subsubsection{The Schweppe-Huber Generalized-M Estimator}

The function $\rho\left(e_{i}(k)\right)$ for the SHGM estimator is given as

$$
\rho\left(e_{i, k}\right)= \begin{cases}\frac{\left(e_{i}(k)\right)^{2}}{2 \sigma_{i}^{2}} & \left|e_{i, k}\right| \leq a_{i} \kappa_{i} \sigma_{i} \\ \frac{a_{i} \kappa_{i}\left|e_{i, k}\right|}{\sigma_{i}}-\frac{a_{i}^{2} \kappa_{i}^{2}}{2} & \text { otherwise }\end{cases}
$$

where $\kappa_{i}$ is the penalty factor chosen specifically to cancel the effect of any existing leverage points in the measurement set and defined as [89]:

$$
\kappa_{i}=\min \left\{1,\left[\frac{\chi_{\nu, p}^{2}}{P S_{i}}\right]\right\}
$$

where $\chi_{\nu, p}^{2}$ is the Chi-square statistics; $\nu$ is the degrees of freedom; and $p$ is probability and is usually taken as $0.975 . P S_{i}$ is the projection statistics for measurement $i$ and its definition is given as [6]

$$
P S_{i}=\max _{H_{k}} \frac{\left|H_{i}^{T} \cdot H_{k}\right|}{\lambda}, \text { for } k=1,2, \ldots, m
$$

where $\lambda=\gamma \cdot \operatorname{lomed}_{i}\left\{\operatorname{lomed}_{j \neq i}\left\{\left|H_{i}^{T} H_{k}+H_{j}^{T} H_{k}\right|\right\}\right\}, 1 \leq i, j, k \leq m$; the factor $\gamma$ is usually chosen as $1.1926[89]$ and $\operatorname{lomed}_{i}\{\cdot\}$ means the low median of the $m$ numbers in the brace. If $P S_{i}>\chi_{\nu, p}^{2}$, then measurement $i$ will be identified as a leverage point. More details about $\kappa_{i}$ and $P S_{i}$ can be found in [89]. When the penalty factor $\kappa_{i}$ in (3.15) is equivalent to 1, then the SHGM estimator reduces to a QL estimator. 
Chapter 3. Variance Analysis of Robust State Estimation in Power Systems using Influence Function ${ }^{1}$

Differentiating $\rho\left(e_{i, k}\right)$ wrt $e_{i}(k)$ and substituting into (3.8) gives

$$
W_{i, k}= \begin{cases}\frac{1}{\sigma_{i}^{2}} & \left|e_{i, k}\right| \leq a_{i} \kappa_{i} \sigma_{i} \\ \frac{a_{i} \kappa_{i}}{\sigma_{i}\left|e_{i, k}\right|} & \text { otherwise }\end{cases}
$$

As a summary, $\rho\left(e_{i, k}\right)$ and their derivatives for the different estimators are shown in Figs. 3.1 and 3.2 where the thresholds are set to $a_{i}=3, b_{i}=4, r_{i}=5$ for the MS estimator; $a_{i}=3$ for $\mathrm{QC}, \mathrm{QL}, \mathrm{SR}$ estimator; and $a_{i}=3, \kappa_{i}=0.7$ for the SHGM estimator. It can be seen that the various robust estimators modified the cost function when measurement residuals are large to reduce the effect of outliers to the state estimates.

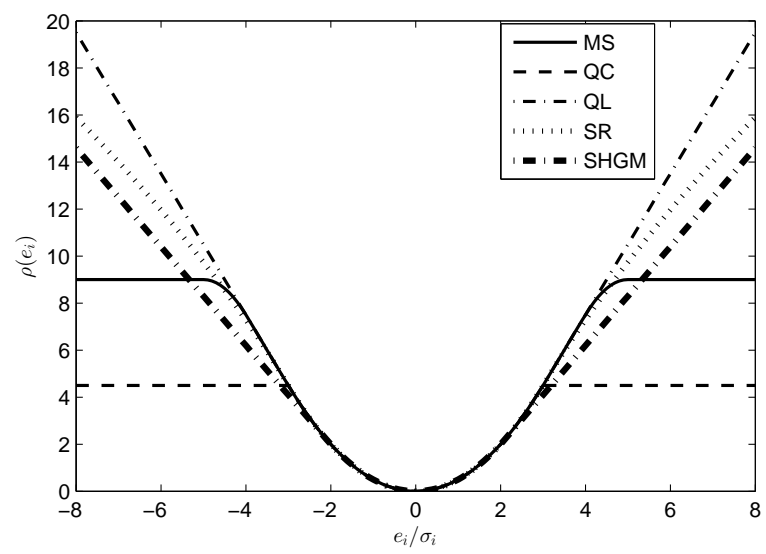

Figure 3.1: $\rho\left(e_{i}\right)$ of the Multiple-Segment (MS with $a_{i}=3, b_{i}=4, r_{i}=5$ ), Quadratic-Constant (QC with $a_{i}=3$ ), Quadratic-Linear (QL with $a_{i}=3$ ), Square-Root (SR with $a_{i}=3$ ) and Schweppe-Huber Generalized-M (SHGM with $\left.a_{i}=3, \kappa_{i}=0.7\right)$ estimator.

\subsubsection{Pseudo-Code}

The steps to implement MS, QC, QL, SR and SHGM estimators can be summarized by the following pseudo-code:

1. Initialization: Choose an initial guess $\hat{x}$; 


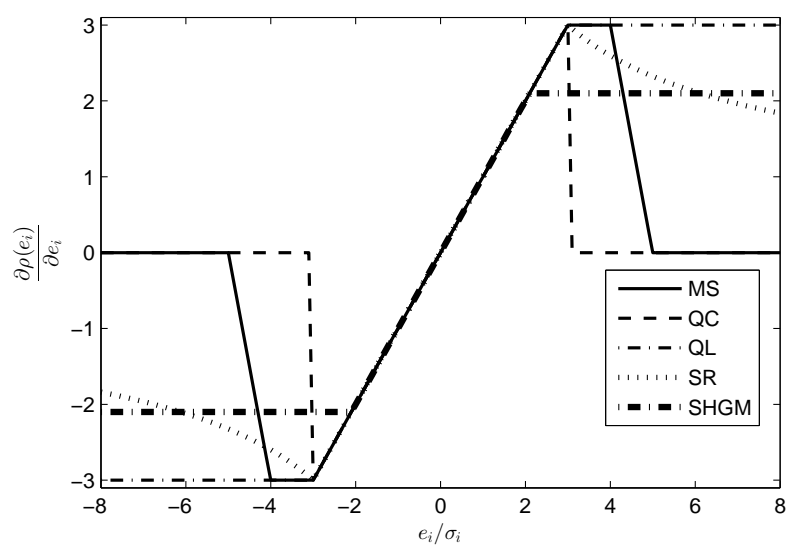

Figure 3.2: The derivative of $\rho\left(e_{i}\right)$ for the Multiple-Segment (MS with $a_{i}=3, b_{i}=$ $4, r_{i}=5$ ), Quadratic-Constant (QC with $a_{i}=3$ ), Quadratic-Linear (QL with $a_{i}=$ 3), Square-Root (SR with $a_{i}=3$ ) and Schweppe-Huber Generalized-M (SHGM with $\left.a_{i}=3, \kappa_{i}=0.7\right)$ estimator.

2. Main procedure:

(a) Iteration index $q \leftarrow 0$;

(b) repeat

i. $q \leftarrow q+1$;

ii. Renew $H$ using (3.4) to form $\bar{H}$;

iii. Calculate $e_{i}$ using (3.6); Renew $W_{i}$ using (3.13), (3.14) or (3.17) to form $W$;

iv. Use (3.12) for the estimation of the state $\hat{x}$;

(c) until $\left(\max \left(\left|\hat{x}^{q}-\hat{x}^{q-1}\right|\right) \leq \delta\right)$ or $q=q_{\max }$.

(d) Use new measurements $Z$ in (3.11) and go back to item (a) so as to obtain $\hat{x}$ in next time step.

Here $\hat{x}^{q}$ is the estimated state vector at the $q$-th iteration. The iteration process will be terminated when the difference $\left(\hat{x}^{q}-\hat{x}^{q-1}\right)$ is less than a specified tolerance $\delta$ or when the index $q$ reaches $q_{\max }$. 
Chapter 3. Variance Analysis of Robust State Estimation in Power Systems using Influence Function ${ }^{1}$

\subsection{Influence Function for Robust PSSE}

\subsubsection{Influence Function Analysis}

The influence function (IF) is given in $[13,25,26]$ as

$$
\operatorname{IF}(\varepsilon)=-\left[\int_{-\infty}^{\infty} \frac{\partial \Psi(\varepsilon)}{\partial x} d F(\varepsilon)\right]^{-1} \Psi(\varepsilon) \approx \hat{x}-\bar{x}
$$

where

$$
\begin{aligned}
\varepsilon \quad & =\left[\epsilon_{1,1} \cdots \epsilon_{m, 1} \cdots \epsilon_{1, N} \cdots \epsilon_{m, N}\right]^{T} \\
d F(\varepsilon) & \\
= & f_{1}\left(\epsilon_{1,1}\right) \times f_{2}\left(\epsilon_{2,1}\right) \times \cdots \times f_{m}\left(\epsilon_{m, 1}\right) \times \cdots \times f_{1}\left(\epsilon_{1, N}\right) \times f_{2}\left(\epsilon_{2, N}\right) \times \cdots \times f_{m}\left(\epsilon_{m, N}\right) \\
& \times d \epsilon_{1,1} \times d \epsilon_{2,1} \times \cdots \times d \epsilon_{m, 1} \times \cdots \times d \epsilon_{1, N} \times d \epsilon_{2, N} \times \cdots \times d \epsilon_{m, N} .
\end{aligned}
$$

since the measurement noise $\epsilon_{i}$ is assumed to be independent and identically distributed. A brief introduction of influence function can be found in Appendix B. Equation (3.18) can be understood intuitively as follows:

The first-order Taylor series expansion of $g(x)$ about the operating point $(\bar{x}, g(\bar{x}))$ is given by

$$
g(x) \approx g(\bar{x})+\left.\frac{d g(x)}{d x}\right|_{x=\bar{x}}(x-\bar{x})
$$

Let $x=\hat{x}, g(\bar{x})=\Psi(\varepsilon), g(x)=0$ and taking expectation of $\frac{d \Psi(\varepsilon)}{d x}$ give (3.18). A formal derivation of the influence function is given in [26].

Using (3.18) as given in $[25,26]$, and $\bar{x}$ is constant in steady state situation, the 
variance of the state estimate can be approximated as

$$
\begin{aligned}
\operatorname{Var}(\hat{x})= & \operatorname{Var}(\bar{x}+\operatorname{IF}(\varepsilon)) \\
= & \int_{-\infty}^{\infty} \operatorname{IF}(\varepsilon) \operatorname{IF}(\varepsilon)^{T} d F(\varepsilon) \\
= & {\left[\int_{-\infty}^{\infty} \frac{\partial \Psi(\varepsilon)}{\partial x} d F(\varepsilon)\right]_{-\infty}^{-1} \Psi(\varepsilon)(\Psi(\varepsilon))^{T} d F(\varepsilon) } \\
& \times\left[\int_{-\infty}^{\infty} \frac{\partial \Psi(\varepsilon)}{\partial \hat{x}} d F(\varepsilon)\right]^{-T}
\end{aligned}
$$

Using (3.10) and let the residue $E$ be given by the noise $\varepsilon$, equation (3.19) gives

$$
\begin{aligned}
\operatorname{Var}(\hat{x})= & {\left[\bar{H}^{T} \int_{-\infty}^{\infty} \frac{\partial W \varepsilon}{\partial \varepsilon} d F(\varepsilon) \bar{H}\right]^{-1} \bar{H}^{T} \int_{-\infty}^{\infty} W \varepsilon \varepsilon^{T} W^{T} d F(\varepsilon) \bar{H} } \\
& \times\left[\bar{H}^{T} \int_{-\infty}^{\infty} \frac{\partial W \varepsilon}{\partial \varepsilon} d F(\varepsilon) \bar{H}\right]^{-T} \\
= & {\left[\bar{H}^{T} \Omega \bar{H}\right]^{-1}\left[\bar{H}^{T} \Lambda \bar{H}\right]\left[\bar{H}^{T} \Omega \bar{H}\right]^{-T} }
\end{aligned}
$$

where

$$
\begin{aligned}
& \Omega=\int_{-\infty}^{\infty} \frac{\partial W \varepsilon}{d \varepsilon} d F(\varepsilon)=\operatorname{diag}\left(\Omega_{1,1}, \ldots, \Omega_{m, 1}, \Omega_{1, N}, \ldots, \Omega_{m, N}\right) \\
& \Lambda=\int_{\infty}^{\infty} W \varepsilon \varepsilon^{T} W^{T} d F(\varepsilon)=\operatorname{diag}\left(\Lambda_{1,1}, \ldots, \Lambda_{m, 1}, \Lambda_{1, N}, \ldots, \Lambda_{m, N}\right)
\end{aligned}
$$

Equation (3.20) is useful. It can be used to calculate the variance of the estimate $\hat{x}$ approximately. The elements of the diagonal matrices $\Omega$ and $\Lambda$ in (3.20) can be obtained as follows: 
Chapter 3. Variance Analysis of Robust State Estimation in Power Systems using Influence Function ${ }^{1}$

\subsubsection{IF for Multiple-Segment Estimator}

Using (3.13), $\Omega_{i, k}$ and $\Lambda_{i, k}$ for the MS estimator is given as

$$
\begin{aligned}
\Omega_{i, k} & =\frac{2}{\sigma_{i}^{2}} \int_{0}^{a_{i} \sigma_{i}} f_{i}\left(\epsilon_{i, k}\right) d \epsilon_{i, k}-\frac{2}{\sigma_{i}^{2}} \int_{b_{i} \sigma_{i}}^{r_{i} \sigma_{i}} \frac{a_{i} f_{i}\left(\epsilon_{i, k}\right)}{r_{i}-b_{i}} d \epsilon_{i, k} \\
\Lambda_{i, k} & =2\left(\int_{0}^{a_{i} \sigma_{i}} \frac{\epsilon_{i, k}^{2}}{\sigma_{i}^{4}} f_{i}\left(\epsilon_{i, k}\right) d \epsilon_{i, k}+\int_{a_{i} \sigma_{i}}^{b_{i} \sigma_{i}} \frac{a_{i}^{2}}{\sigma_{i}^{2}} f_{i}\left(\epsilon_{i, k}\right) d \epsilon_{i, k}\right. \\
& \left.+\int_{b_{i} \sigma_{i}}^{r_{i} \sigma_{i}} \frac{a_{i}^{2}\left(r_{i} \sigma_{i}-\epsilon_{i, k}\right)^{2}}{\sigma_{i}^{4}\left(r_{i}-b_{i}\right)^{2}} f_{i}\left(\epsilon_{i, k}\right) d \epsilon_{i, k}\right)
\end{aligned}
$$

\subsubsection{IF for Square-Root Estimator}

Using (3.14), $\Omega_{i, k}$ and $\Lambda_{i, k}$ for the SR estimator are given as

$$
\begin{aligned}
& \Omega_{i, k}=\frac{2}{\sigma_{i}^{2}} \int_{0}^{a_{i} \sigma_{i}} f_{i}\left(\epsilon_{i, k}\right) d \epsilon_{i, k}-\int_{a_{i} \sigma_{i}}^{\infty} \sqrt{\frac{a_{i}^{3}}{\sigma_{i}\left|\epsilon_{i, k}\right|^{3}}} f_{i}\left(\epsilon_{i, k}\right) d \epsilon_{i, k} \\
& \Lambda_{i, k}=\int_{0}^{a_{i} \sigma_{i}} \frac{2 \epsilon_{i, k}^{2}}{\sigma_{i}^{4}} f_{i}\left(\epsilon_{i, k}\right) d \epsilon_{i, k}+\int_{a_{i} \sigma_{i}}^{\infty} \frac{2 a_{i}^{3}}{\sigma_{i}\left|\epsilon_{i, k}\right|} f_{i}\left(\epsilon_{i, k}\right) d \epsilon_{i, k}
\end{aligned}
$$

\subsubsection{IF for Schweppe-Huber Generalized-M Estimator}

Substituting (3.17) into (3.21) and (3.22) gives

$$
\begin{aligned}
& \Omega_{i, k}=\frac{2}{\sigma_{i}^{2}} \int_{0}^{a_{i} \kappa_{i} \sigma_{i}} f_{i}\left(\epsilon_{i, k}\right) d \epsilon_{i, k} \\
& \Lambda_{i, k}=2\left(\int_{0}^{a_{i} \kappa_{i} \sigma_{i}} \frac{\epsilon_{i, k}^{2}}{\sigma_{i}^{4}} f_{i}\left(\epsilon_{i, k}\right) d \epsilon_{i, k}+\int_{a_{i} \kappa_{i} \sigma_{i}}^{\infty} \frac{a_{i}^{2} \kappa_{i}^{2}}{\sigma_{i}^{2}} f_{i}\left(\epsilon_{i, k}\right) d \epsilon_{i, k}\right)
\end{aligned}
$$

\subsubsection{The Weighted-Least-Squares Connection}

To put things in perspective, this section connects the equations derived in this chapter with the well-known variance equation of weighted-least-squares (WLS) estimation. 
Equation (3.20) derived from IF approximation can also give the variance of the WLS estimator when the noise $\epsilon_{i}$ is associated with the Gaussian pdf

$$
f_{i}\left(\epsilon_{i}\right)=\frac{1}{\sqrt{2 \pi \sigma_{i}^{2}}} \exp \left(-\frac{\epsilon_{i}^{2}}{2 \sigma_{i}^{2}}\right)
$$

and $a_{i} \rightarrow \infty$. Now (3.23) and (3.25) reduce to

$$
\Omega_{i}=\frac{2}{\sigma_{i}^{2}} \int_{0}^{\infty} \frac{1}{\sqrt{2 \pi \sigma_{i}^{2}}} \exp \left(-\frac{\epsilon_{i}^{2}}{2 \sigma_{i}^{2}}\right) d \epsilon_{i}=\frac{1}{\sigma_{i}^{2}}
$$

Equations (3.24) and (3.26) reduce to

$$
\Lambda_{i}=2 \int_{0}^{\infty} \frac{\epsilon_{i}^{2}}{\sigma_{i}^{4}} \frac{1}{\sqrt{2 \pi \sigma_{i}^{2}}} \exp \left(-\frac{\epsilon_{i}^{2}}{2 \sigma_{i}^{2}}\right) d \epsilon_{i}=\frac{1}{\sigma_{i}^{2}}
$$

Substituting (3.29) and (3.30) into (3.20) gives the well-known WLS variance equation $[26]$

$$
\operatorname{Var}(\hat{x})=\left[\bar{H}^{T} \operatorname{diag}\left(\frac{1}{\sigma_{1}^{2}}, \ldots, \frac{1}{\sigma_{m}^{2}}, \ldots, \frac{1}{\sigma_{1}^{2}}, \ldots, \frac{1}{\sigma_{m}^{2}}\right) \bar{H}\right]^{-1}
$$

\subsection{Simulation Results}

A textbook example [3] on a simple power system with WLS estimation in the presence of Gaussian noise is given in Example 1 to illustrate the detail calculations for variance equation (3.20). State estimation with PMU measurements on the IEEE 14-bus system is given in Examples 2, 3 and on a larger IEEE 30-bus system in Example 4. Example 2 shows how equation (3.20) can be used to express the variance of a state estimate as a function of measurement variances enabling the selection of sensors for specified estimation precision. Example 3 shows how the proposed variance equation (3.20) can be used to design an optimal estimator. The 
Chapter 3. Variance Analysis of Robust State Estimation in Power Systems using Influence Function ${ }^{1}$

Table 3.1: Parameters of estimators and noises used in the IEEE 14-bus system.

\begin{tabular}{|c|c|c|c|c|c|}
\hline Example & 1 & \multicolumn{2}{|c|}{2} & \multicolumn{2}{|c|}{3} \\
\hline Model & $\begin{array}{c}4 \text { Measurements, } \\
3 \text { States }\end{array}$ & \multicolumn{4}{|c|}{$\begin{array}{c}\text { IEEE 14-bus System } \\
\text { with PMU Measurements }\end{array}$} \\
\hline $\begin{array}{c}\text { Measure- } \\
\text { ment } i\end{array}$ & $1, \ldots, 4$ & $1, \ldots, 12$ & $13, \ldots, 58$ & $1, \ldots, 12$ & $13, \ldots, 58$ \\
\hline $\begin{array}{l}\text { Normal } \\
\text { Noise }\end{array}$ & $\begin{array}{l}{ }^{*} N(0.02) \\
N(0.03) \\
N(0.03) \\
N(0.04)\end{array}$ & $\begin{array}{c}0.99 \times \\
N(0.006)\end{array}$ & $\begin{array}{c}0.97 \times \\
N(0.003)\end{array}$ & \multirow[t]{2}{*}{$t_{3}$} & \multirow[t]{2}{*}{$t_{5}$} \\
\hline Outliers & - & $\begin{array}{c}0.01 \times \\
N(0.06)\end{array}$ & $\begin{array}{l}{ }^{\dagger} 0.03 \times \\
U(0.03)\end{array}$ & & \\
\hline $\begin{array}{l}{ }^{\ddagger} \text { Esti- } \\
\text { mator }\end{array}$ & $\begin{array}{c}\text { WLS } \\
\text { or } \\
\operatorname{MS}(5,6,7)\end{array}$ & $\begin{array}{c}\mathrm{QC}(2.5) \\
\mathrm{QL}(2.5) \\
\mathrm{SR}(2.5) \\
\mathrm{SHGM}(2.5) \\
\mathrm{MS}(2.5,3.5, \\
4.5)\end{array}$ & $\begin{array}{c}\mathrm{QC}(3) \\
\mathrm{QL}(3) \\
\mathrm{SR}(3) \\
\mathrm{SHGM}(3) \\
\mathrm{MS}(3,4, \\
5)\end{array}$ & $\begin{array}{c}\mathrm{MS}(2.5,3.5, \\
4.5) \\
\operatorname{MS}(0.6,1.5, \\
6)\end{array}$ & $\begin{array}{c}\operatorname{MS}(3,4,5) \\
\operatorname{MS}(0.8,2.5 \\
6)\end{array}$ \\
\hline Fig. & 3.3 & \multicolumn{2}{|c|}{$3.5,3.6$} & \multicolumn{2}{|c|}{3.7} \\
\hline Table & - & \multicolumn{2}{|c|}{$3.2,3.3$} & \multicolumn{2}{|c|}{3.4} \\
\hline
\end{tabular}

${ }^{*} N\left(\sigma_{i}\right)=\frac{1}{\sqrt{2 \pi \sigma_{i}^{2}}} \exp \left(-\frac{\epsilon_{i}^{2}}{2 \sigma_{i}^{2}}\right)$.

${ }^{\dagger} U\left(\sigma_{i}\right)=\frac{1}{2 \sigma_{i}}$ for $-\sigma_{i} \leq \epsilon_{i} \leq \sigma_{i}$.

$\ddagger$ Parameter $a_{i}$ is given in the bracket of $\mathrm{QC}\left(a_{i}\right), \mathrm{QL}\left(a_{i}\right), \operatorname{SR}\left(a_{i}\right)$ and $\operatorname{SHGM}\left(a_{i}\right)$ while parameters $a_{i}, b_{i}, r_{i}$ are given in the bracket of $\operatorname{MS}\left(a_{i}, b_{i}, r_{i}\right)$.

variance equation (3.20) takes much lesser time to get the optimal parameters, compared with Monte Carlo methods. Example 4 shows the variances calculated by equation (3.20) can be used to approximate the variances obtained by Monte Carlo simulation results, where the maximum percentage error between them is small.

For easy reference, the parameters of the robust state estimators and probability density functions of the "normal" noise and outliers are summarized in Tables 3.1 and 3.5 . 


\subsubsection{Example 1: Four Measurements, Three States}

Consider Fig. 3.3, where voltage measurements $V_{1}, V_{2}$, real and reactive power flow measurements $P_{21}, Q_{21}$ are given. Gaussian noise is assumed, Bus 1 is taken as the slack bus and $\theta_{2}$ is the phase angle of Bus 2. The measurement model is given as

$$
y_{k}=h\left(x_{k}\right)+\epsilon_{k}
$$

where

$$
\begin{aligned}
& x_{k}=\left[\begin{array}{lll}
V_{1, k} & V_{2, k} & \theta_{2, k}
\end{array}\right]^{T} \\
& y_{k}=\left[\begin{array}{llll}
V_{1, k} & V_{2, k} & P_{21, k} & Q_{21, k}
\end{array}\right]^{T} \\
& \epsilon_{k}=\left[\begin{array}{llll}
\epsilon_{1, k} & \epsilon_{2, k} & \epsilon_{3, k} & \epsilon_{4, k}
\end{array}\right]^{T}
\end{aligned}
$$

The vector function $h\left(x_{k}\right)$ is given as:

$$
\begin{aligned}
& h_{1}\left(x_{k}\right)=V_{1, k} \\
& h_{2}\left(x_{k}\right)=V_{2, k} \\
& h_{3}\left(x_{k}\right)=15.0 V_{1, k} V_{2, k} \sin \left(\theta_{2, k}\right) \\
& h_{4}\left(x_{k}\right)=15.0 V_{2, k}^{2}-15.0 V_{1, k} V_{2, k} \cos \left(\theta_{2, k}\right)
\end{aligned}
$$


Chapter 3. Variance Analysis of Robust State Estimation in Power Systems using Influence Function ${ }^{1}$

The Jacobian matrix in [3] is given as

$$
H=\left[\begin{array}{ccc}
1.00 & 0 & 0 \\
0 & 1.00 & 0 \\
-1.57 & -1.57 & 14.83 \\
-14.88 & 15.05 & -1.56
\end{array}\right]
$$

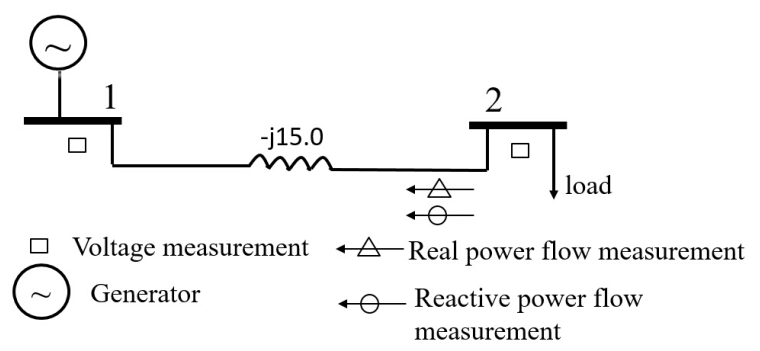

Figure 3.3: A simple example for PSSE with voltage measurements $V_{1}, V_{2}$, real and reactive power flow measurements $P_{21}, Q_{21}[3]$.

The variance formula for the WLS estimator in [3] gives

$$
\operatorname{Var}(\hat{x})=\left(H^{T} W H\right)^{-1}=\left[\begin{array}{ccc}
2.76 & 2.76 & 0.58 \\
2.76 & 2.84 & 0.60 \\
0.58 & 0.60 & 0.17
\end{array}\right] \times 10^{-4}
$$

where

$$
W=\operatorname{diag}\left(\frac{1}{\sigma_{1}^{2}}, \frac{1}{\sigma_{2}^{2}}, \frac{1}{\sigma_{3}^{2}}, \frac{1}{\sigma_{4}^{2}}\right)
$$

and the standard deviation of the Gaussian noise $\sigma_{1}=0.02, \sigma_{2}=\sigma_{3}=0.03$, $\sigma_{4}=0.04$

The results in (3.33) given in [3] can also be obtained from (3.20) as follows. The variance of WLS estimator can be obtained by choosing the threshold parameter " $a_{i}$ " large [6] such that the pdf $f_{i}\left(\epsilon_{i}\right)$ is zero outside the threshold " $a_{i} \sigma_{i}$ ". Threshold 
parameters " $b_{i}$ " and " $r_{i}$ " can be chosen arbitrarily as $r_{i}>b_{i}>a_{i}$ and are outside the noise distribution. For the Gaussian distribution, $a_{i}=5$ is a good choice.

Using $a_{1}=5, b_{1}=6, r_{1}=7$ in (3.21) and (3.22) give

$$
\begin{aligned}
\Omega_{1,1} & =\frac{2}{\sigma_{1}^{2}} \int_{0}^{5 \sigma_{1}} f_{1}\left(\epsilon_{1}\right) d \epsilon_{1}-\frac{2}{\sigma_{1}^{2}} \int_{6 \sigma_{1}}^{7 \sigma_{1}} \frac{5 f_{1}\left(\epsilon_{1}\right)}{7-6} d \epsilon_{1}=2500 \\
\Lambda_{1,1} & =2\left(\int_{0}^{5 \sigma_{1}} \frac{\epsilon_{1}^{2}}{\sigma_{1}^{4}} f_{1}\left(\epsilon_{1}\right) d \epsilon_{1}+\int_{5 \sigma_{1}}^{6 \sigma_{1}} \frac{5^{2}}{\sigma_{1}^{2}} f_{1}\left(\epsilon_{1}\right) d \epsilon_{1}+\int_{6 \sigma_{1}}^{7 \sigma_{1}} \frac{5^{2}\left(7 \sigma_{1}-\epsilon_{1}\right)^{2}}{\sigma_{1}^{4}(7-6)^{2}} f_{1}\left(\epsilon_{1}\right) d \epsilon_{1}\right) \\
& =2500
\end{aligned}
$$

where $f_{1}\left(\epsilon_{1}\right)$ is the pdf of the zero-mean Gaussian distribution with standard deviation $\sigma_{1}$.

Note that the definite integral in $\Omega_{1}$ and $\Lambda_{1}$ can be calculated easily with the help of the Gauss error function [90]

$$
\operatorname{erf}(x)=\frac{2}{\sqrt{\pi}} \int_{0}^{x} e^{-t^{2}} d t
$$

Similar calculations give $\Omega_{2,1}=\Omega_{3,1}=1111 ; \Omega_{4,1}=625 ; \Lambda_{2,1}=\Lambda_{3,1}=1111 ;$ and $\Lambda_{4,1}=625$. Variance equation (3.20) then gives the same results in (3.33). Other than the WLS estimator with the usual Gaussian noise assumption, formulas for the variance of the estimates from the QC, QL, MS, SR and SHGM estimators with non-Gaussian noise for PSSE are not given in the literature and this is where (3.20) derived in this thesis becomes useful.

\subsubsection{Example 2: The IEEE 14-bus System with PMU mea- surements}

The PMUs are placed according to [91], as shown in Fig. 3.4. Since PMUs are used to take measurements, the measurement model in (3.1) is applicable. The 
Chapter 3. Variance Analysis of Robust State Estimation in Power Systems using Influence Function ${ }^{1}$

matrix $H$ is calculated according to [92]. There are $n=28$ states in the vector $x=\left[V_{1}^{r} V_{2}^{r} \cdots V_{14}^{r} V_{1}^{i m} \cdots V_{14}^{i m}\right]^{T}$. Fifty-eight measurements, $z_{i}, i=1, \ldots, 58$, comprising 12 voltages $(i=1, \ldots, 12)$ and 46 currents $(i=13, \ldots, 58)$ are taken at each time instance $k$ and $N=3$ sets of measurements i.e. $k=1,2,3$ are used to give one set of estimates. Measurement outliers are removed by the robust estimator when they are outside the estimator cut-off " $r$ ". There could be insufficient data for the equations to solve for the estimates if only $N=1$ set of measurements is used.

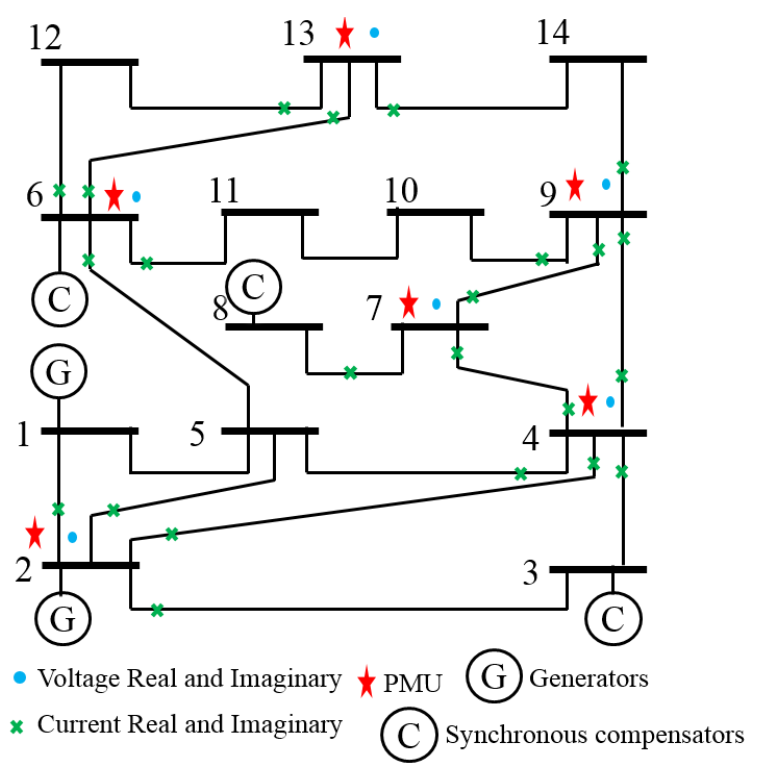

Figure 3.4: The IEEE 14-bus system installed with PMUs.

For the voltage measurements $z_{i}, i=1, \ldots, 12$, the parameters for the QC, QL, SR and SHGM estimators are chosen as $a_{i}=2.5[31,32]$. The threshold parameters " $b_{i}$ " and " $r_{i}$ " follows the relationship $r_{i}>b_{i}>a_{i}$, so the parameters for the MS estimator are chosen as $a_{i}=2.5, b_{i}=3.5, r_{i}=4.5$. The penalty $\kappa_{i}, i=1, \ldots, 58$ of the SHGM estimator is calculated by (3.16). The standard deviation for measurement from PMU is between 0.0005 and 0.01 [93]. Here, the noise $\epsilon_{i}$ is associated with the pdf

$$
f_{i}\left(\epsilon_{i}\right)=\frac{0.99}{\sqrt{2 \pi \sigma_{i}^{2}}} \exp \left(-\frac{\epsilon_{i}^{2}}{2 \sigma_{i}^{2}}\right)+\frac{0.01}{\sqrt{2 \pi\left(10 \sigma_{i}\right)^{2}}} \exp \left(-\frac{\epsilon_{i}^{2}}{2\left(10 \sigma_{i}\right)^{2}}\right)
$$


where $\sigma_{i}=0.006$. The pdf in the form of a Gaussian mixture distribution in (3.34) is also used in [26]. The first term in the above pdf represents the $99 \%$ of "normal" noise while the second term represents outliers by the $1 \%$ Gaussian noise with standard deviation $10 \sigma_{i}$.

For the current measurements $z_{i}, i=13, \ldots, 58$, the parameters for the $\mathrm{QC}, \mathrm{QL}$, SR and SHGM estimators are chosen as $a_{i}=3[31,32]$ while the parameters for the MS estimator are $a_{i}=3, b_{i}=4, r_{i}=5$. The noise $\epsilon_{i}$ is associated with the pdf

$$
f_{i}\left(\epsilon_{i}\right)= \begin{cases}\frac{0.97}{\sqrt{2 \pi \sigma_{i}^{2}}} \exp \left(-\frac{\epsilon_{i}^{2}}{2 \sigma_{i}^{2}}\right)+\frac{0.03}{2 \times 10 \sigma_{i}} & \left|\epsilon_{i}\right| \leq 10 \sigma_{i} \\ \frac{0.97}{\sqrt{2 \pi \sigma_{i}^{2}}} \exp \left(-\frac{\epsilon_{i}^{2}}{2 \sigma_{i}^{2}}\right) & \text { otherwise }\end{cases}
$$

where $\sigma_{i}=0.003$. The pdf in the form of a mixture distribution in (3.35) is also given in [32]. The $3 \%$ of uniform distribution $\frac{0.03}{2 \times 10 \sigma_{i}}$ in (3.35) is useful for modeling initial conditions, disturbances and measurement errors that are equally likely to occur anywhere within a given interval. In the study of outliers, the "normal" noise is typically represented by the Gaussian distribution, while the outliers by Gaussian with a larger standard deviation or non-Gaussian distributions [26,32].

In this example, the $\mathrm{QC}, \mathrm{QL}, \mathrm{SR}, \mathrm{MS}$ and SHGM robust state estimators are used to estimate the states of the IEEE 14-bus system. The non-robust estimator WLS is taken as a special MS (when parameters $a_{i}$ tends to $\infty$ ). Since the detail calculations are already illustrated through a simple system in Example 1, the results of the calculations are directly given in Tables 3.2 and 3.3. The variances calculated from variance equation (3.20) are given under column "Eq. (3.20)". To give an idea of the accuracy, the variance calculated from (3.20) is compared with the variance obtained from 10,000 simulation runs and the results are also given in Tables 3.2 and 3.3 under column "Simulation". The maximum percentage error between the variances from simulation and from (3.20) given under column "\% Error" is less than $5 \%$ which in practice should be good enough. Furthermore, when the outliers 
Chapter 3. Variance Analysis of Robust State Estimation in Power Systems using Influence Function ${ }^{1}$

increase to $5 \%$,

$$
f_{i}\left(\epsilon_{i}\right)=\frac{0.95}{\sqrt{2 \pi \sigma_{i}^{2}}} \exp \left(-\frac{\epsilon_{i}^{2}}{2 \sigma_{i}^{2}}\right)+\frac{0.05}{\sqrt{2 \pi\left(10 \sigma_{i}\right)^{2}}} \exp \left(-\frac{\epsilon_{i}^{2}}{2\left(10 \sigma_{i}\right)^{2}}\right)
$$

where $\sigma_{i}=0.006, i=1, \ldots 12$, and

$$
f_{i}\left(\epsilon_{i}\right)= \begin{cases}\frac{0.95}{\sqrt{2 \pi \sigma_{i}^{2}}} \exp \left(-\frac{\epsilon_{i}^{2}}{2 \sigma_{i}^{2}}\right)+\frac{0.05}{2 \times 10 \sigma_{i}} & \left|\epsilon_{i}\right| \leq 10 \sigma_{i} \\ \frac{0.95}{\sqrt{2 \pi \sigma_{i}^{2}}} \exp \left(-\frac{\epsilon_{i}^{2}}{2 \sigma_{i}^{2}}\right) & \text { otherwise }\end{cases}
$$

where $\sigma_{i}=0.003, i=13, \ldots 58$, the maximum percentage error increases to $7 \%$. In this case, the approximate solutions from the variance equations can still be used as good initial conditions in simulation runs when more accurate results are desired.

Equation (3.20) can also be used to obtain the variance of a state estimate as a function of measurement variances. Consider the MS estimation. When $\sigma_{i}=0.006$, $i=1, \ldots, 12$, and $\sigma_{i}=0.003, i=13, \ldots, 58$, equation $(3.20)$ gives $\operatorname{Var}\left(\hat{V}_{12}^{r}\right)=$ $2.26 \times 10^{-6}$ in Table 3.3 (see Row " $\hat{V}_{12}^{r}$ ", Column "MS-Eq. (3.20)"). If it is not given that $\sigma_{3}=0.006$, then $\sigma_{3}$ can be left as a variable and (3.20) gives

$$
\operatorname{Var}\left(\hat{V}_{12}^{r}\right)=\frac{6.27 \times 10^{5} \sigma_{3}^{4}+4.93 \sigma_{3}^{2}+1.90 \times 10^{-6}}{\left(4.82 \times 10^{5} \sigma_{3}^{2}+3.61\right)^{2}}
$$

Equation (3.36) is plotted as the solid-line in Fig. 3.5. The equations for the variances of the other 27 state estimates as a function of $\sigma_{3}$ were likewise obtained and plotted in the figure as dashed-lines. Fig. 3.5 now summarizes for the MS estimation how the variances of all 28 state estimates are affect by $\sigma_{3}$. Instead of $\sigma_{3}$, obviously variance equation (3.20) can also give $\operatorname{Var}\left(\hat{V}_{12}^{r}\right)$ as a function of the other $\sigma_{i}$ as shown in Fig. 3.6 where (3.36) is again plotted as the solid-line. The figures can help us select sensors to give specified estimation precision. For example if the variance of the state $V_{12}^{r}$, $\operatorname{Var}\left(\hat{V}_{12}^{r}\right) \leq 1.6 \times 10^{-6}$ is specified then the solid-line 
Table 3.2: Variances of the state estimates (WLS, QC, QL) in the IEEE 14-bus system using the linear measurement model in (3.1).

\begin{tabular}{|c|c|c|c|c|c|c|c|c|c|}
\hline & \multicolumn{3}{|c|}{$\begin{array}{c}\text { WLS } \\
a_{i} \rightarrow \infty, b_{i} \rightarrow \infty \\
r_{i} \rightarrow \infty, i=1, \ldots, 58\end{array}$} & \multicolumn{3}{|c|}{$\begin{array}{l}\mathrm{QC} \\
a_{i}=2.5, b_{i} \rightarrow a_{i}, \\
r_{i} \rightarrow a_{i}, i=1, \ldots, 12 ; \\
a_{i}=3, b_{i} \rightarrow a_{i}, \\
r_{i} \rightarrow a_{i}, i=13, \ldots, 58 .\end{array}$} & \multicolumn{3}{|c|}{$\begin{array}{l}\mathrm{QL} \\
a_{i}=2.5, b_{i} \rightarrow \infty, \\
r_{i} \rightarrow \infty, i=1, \ldots, 12 \\
a_{i}=3, b_{i} \rightarrow \infty, \\
r_{i} \rightarrow \infty, i=13, \ldots, 58\end{array}$} \\
\hline & $\begin{array}{l}\text { Simu } \\
\text { lation }\end{array}$ & $\begin{array}{c}\text { Eq. } \\
(3.20)\end{array}$ & $\begin{array}{c}\% \\
\text { Error }\end{array}$ & $\begin{array}{l}\text { Simu } \\
\text { lation }\end{array}$ & $\begin{array}{c}\text { Eq. } \\
(3.20)\end{array}$ & $\begin{array}{c}\% \\
\text { Error }\end{array}$ & $\begin{array}{l}\text { Simu } \\
\text { lation }\end{array}$ & $\begin{array}{c}\text { Eq. } \\
(3.20)\end{array}$ & $\begin{array}{c}\% \\
\text { Error }\end{array}$ \\
\hline$\hat{V}_{1}^{r}$ & 4.14 & 4.08 & -1.4 & 2.22 & 2.31 & 4.1 & 2.19 & 2.20 & 0.5 \\
\hline$\hat{V}_{2}^{r}$ & 4.14 & 4.07 & -1.7 & 2.22 & 2.31 & 4.1 & 2.17 & 2.19 & 0.9 \\
\hline$\hat{V}_{3}^{r}$ & 4.17 & 4.11 & -1.4 & 2.24 & 2.33 & 4.0 & 2.22 & 2.22 & 0.0 \\
\hline$\hat{V}_{4}^{r}$ & 4.08 & 4.03 & -1.2 & 2.19 & 2.28 & 4.1 & 2.15 & 2.16 & 0.5 \\
\hline$\hat{V}_{5}^{r}$ & 4.09 & 4.03 & -1.5 & 2.19 & 2.28 & 4.1 & 2.15 & 2.16 & 0.5 \\
\hline$\hat{V}_{6}^{r}$ & 4.13 & 4.10 & -0.7 & 2.23 & 2.32 & 4.0 & 2.21 & 2.21 & 0.0 \\
\hline$\hat{V}_{7}^{r}$ & 4.09 & 4.05 & -1.0 & 2.20 & 2.29 & 4.1 & 2.16 & 2.17 & 0.5 \\
\hline$\hat{V}_{8}^{r}$ & 4.27 & 4.23 & -0.9 & 2.30 & 2.39 & 3.9 & 2.29 & 2.29 & 0.0 \\
\hline$\hat{V}_{9}^{r}$ & 4.10 & 4.06 & -1.0 & 2.21 & 2.30 & 4.1 & 2.15 & 2.18 & 1.4 \\
\hline$\hat{V}_{10}^{r}$ & 4.14 & 4.11 & -0.7 & 2.23 & 2.33 & 4.5 & 2.18 & 2.21 & 1.4 \\
\hline$\hat{V}_{11}^{r}$ & 4.39 & 4.39 & 0.0 & 2.39 & 2.48 & 3.8 & 2.41 & 2.39 & -0.8 \\
\hline$\hat{V}_{12}^{r}$ & 4.38 & 4.35 & -0.7 & 2.37 & 2.46 & 3.8 & 2.36 & 2.37 & 0.4 \\
\hline$\hat{V}_{13}^{r}$ & 4.16 & 4.13 & -0.7 & 2.25 & 2.34 & 4.0 & 2.21 & 2.22 & 0.5 \\
\hline$\hat{V}_{14}^{r}$ & 4.36 & 4.33 & -0.7 & 2.38 & 2.45 & 2.9 & 2.36 & 2.35 & -0.4 \\
\hline$\hat{V}_{1}^{i m}$ & 3.95 & 4.08 & 3.3 & 2.26 & 2.31 & 2.2 & 2.23 & 2.20 & -1.3 \\
\hline$\hat{V}_{2}^{i m}$ & 3.95 & 4.07 & 3.0 & 2.25 & 2.31 & 2.7 & 2.22 & 2.19 & -1.4 \\
\hline$\hat{V}_{3}^{i m}$ & 3.97 & 4.11 & 3.5 & 2.24 & 2.33 & 4.0 & 2.26 & 2.22 & -1.8 \\
\hline$\hat{V}_{4}^{i m}$ & 3.90 & 4.03 & 3.3 & 2.21 & 2.28 & 3.2 & 2.18 & 2.16 & -0.9 \\
\hline$\hat{V}_{5}^{i m}$ & 3.90 & 4.03 & 3.3 & 2.21 & 2.28 & 3.2 & 2.18 & 2.16 & -0.9 \\
\hline$\hat{V}_{6}^{i m}$ & 3.98 & 4.10 & 3.0 & 2.26 & 2.32 & 2.7 & 2.23 & 2.21 & -0.9 \\
\hline$\hat{V}_{7}^{i m}$ & 3.92 & 4.05 & 3.3 & 2.23 & 2.29 & 2.7 & 2.21 & 2.17 & -1.8 \\
\hline$\hat{V}_{8}^{i m}$ & 4.10 & 4.23 & 3.2 & 2.33 & 2.39 & 2.6 & 2.33 & 2.29 & -1.7 \\
\hline$\hat{V}_{9}^{i m}$ & 3.94 & 4.06 & 3.0 & 2.23 & 2.30 & 3.1 & 2.21 & 2.18 & -1.4 \\
\hline$\hat{V}_{10}^{i m}$ & 4.00 & 4.11 & 2.7 & 2.26 & 2.33 & 3.1 & 2.25 & 2.21 & -1.8 \\
\hline$\hat{V}_{11}^{i m}$ & 4.26 & 4.39 & 3.1 & 2.42 & 2.48 & 2.5 & 2.47 & 2.39 & -3.2 \\
\hline$\hat{V}_{12}^{i m}$ & 4.25 & 4.35 & 2.4 & 2.42 & 2.46 & 1.7 & 2.40 & 2.37 & -1.2 \\
\hline$\hat{V}_{13}^{i m}$ & 4.00 & 4.13 & 3.3 & 2.28 & 2.34 & 2.6 & 2.23 & 2.22 & -0.4 \\
\hline$\hat{V}_{14}^{i m}$ & 4.20 & 4.33 & 3.1 & 2.38 & 2.45 & 2.9 & 2.36 & 2.35 & -0.4 \\
\hline
\end{tabular}

Variances unit: $\times 10^{-6}$;

$\%$ Error $=($ Eq. $(3.20)-$ Simulation $) /$ Simulation $\times 100 \%$. 
Chapter 3. Variance Analysis of Robust State Estimation in Power Systems using Influence Function ${ }^{1}$

Table 3.3: Variances of the state estimates (SR, MS, SHGM) in the IEEE 14-bus system using the linear measurement model in (3.1).

\begin{tabular}{|c|c|c|c|c|c|c|c|c|c|}
\hline & \multicolumn{3}{|c|}{$\begin{array}{c}\mathrm{SR} \\
a_{i}=2.5, i=1, \ldots, 12 \\
a_{i}=3, i=13, \ldots, 58\end{array}$} & \multicolumn{3}{|c|}{\begin{tabular}{l}
\multicolumn{1}{c}{$\mathrm{MS}$} \\
$a_{i}=2.5, b_{i}=3.5$, \\
$r_{i}=4.5, i=1, \ldots, 12$ \\
$a_{i}=3, b_{i}=4, r_{i}=5$, \\
$i=13, \ldots, 58$
\end{tabular}} & \multicolumn{3}{|c|}{$\begin{array}{c}\text { SHGM } \\
a_{i}=2.5, i=1, \ldots, 12 ; \\
a_{i}=3, i=13, \ldots, 58 .\end{array}$} \\
\hline & $\begin{array}{l}\text { Simu } \\
\text { lation }\end{array}$ & $\begin{array}{c}\text { Eq. } \\
(3.20)\end{array}$ & $\begin{array}{c}\% \\
\text { Error }\end{array}$ & $\begin{array}{l}\text { Simu } \\
\text { lation }\end{array}$ & $\begin{array}{c}\text { Eq. } \\
(3.20)\end{array}$ & $\begin{array}{c}\% \\
\text { Error }\end{array}$ & $\begin{array}{l}\text { Simu } \\
\text { lation }\end{array}$ & $\begin{array}{c}\text { Eq. } \\
(3.20)\end{array}$ & $\begin{array}{c}\% \\
\text { Error }\end{array}$ \\
\hline$\hat{V}_{1}^{r}$ & 2.16 & 2.13 & -1.4 & 2.13 & 2.11 & -0.9 & 2.22 & 2.22 & 0.0 \\
\hline$\hat{V}_{2}^{r}$ & 2.15 & 2.13 & -0.9 & 2.12 & 2.10 & -0.9 & 2.21 & 2.21 & 0.0 \\
\hline$\hat{V}_{3}^{r}$ & 2.18 & 2.15 & -1.4 & 2.16 & 2.12 & -1.9 & 2.24 & 2.26 & 0.9 \\
\hline$\hat{V}_{4}^{r}$ & 2.12 & 2.10 & -0.9 & 2.10 & 2.07 & -1.4 & 2.18 & 2.17 & -0.4 \\
\hline$\hat{V}_{5}^{r}$ & 2.12 & 2.10 & -0.9 & 2.10 & 2.08 & -1.0 & 2.18 & 2.18 & 0.0 \\
\hline$\hat{V}_{6}^{r}$ & 2.16 & 2.15 & -0.5 & 2.14 & 2.12 & -0.9 & 2.23 & 2.25 & 0.9 \\
\hline$\hat{V}_{7}^{r}$ & 2.13 & 2.11 & -0.9 & 2.10 & 2.09 & -0.5 & 2.20 & 2.19 & -0.5 \\
\hline$\hat{V}_{8}^{r}$ & 2.25 & 2.22 & -1.3 & 2.22 & 2.19 & -1.4 & 2.36 & 2.36 & 0.0 \\
\hline$\hat{V}_{9}^{r}$ & 2.15 & 2.12 & -1.4 & 2.11 & 2.09 & -0.9 & 2.21 & 2.20 & -0.5 \\
\hline$\hat{V}_{10}^{r}$ & 2.18 & 2.15 & -1.4 & 2.14 & 2.12 & -0.9 & 2.24 & 2.25 & 0.3 \\
\hline$\hat{V}_{11}^{r}$ & 2.33 & 2.31 & -0.9 & 2.31 & 2.28 & -1.3 & 2.51 & 2.51 & 0.0 \\
\hline$\hat{V}_{12}^{r}$ & 2.33 & 2.29 & -1.7 & 2.28 & 2.26 & -0.9 & 2.48 & 2.47 & -0.4 \\
\hline$\hat{V}_{13}^{r}$ & 2.17 & 2.16 & -0.5 & 2.15 & 2.13 & -0.9 & 2.23 & 2.27 & 1.8 \\
\hline$\hat{V}_{14}^{r}$ & 2.29 & 2.28 & -0.4 & 2.25 & 2.24 & -0.4 & 2.41 & 2.45 & 1.7 \\
\hline$\hat{V}_{1}^{i m}$ & 2.16 & 2.13 & -1.4 & 2.11 & 2.11 & 0.0 & 2.27 & 2.22 & -2.2 \\
\hline$\hat{V}_{2}^{i m}$ & 2.15 & 2.13 & -0.9 & 2.11 & 2.10 & -0.5 & 2.27 & 2.21 & -2.6 \\
\hline$\hat{V}_{3}^{i m}$ & 2.18 & 2.15 & -1.4 & 2.13 & 2.12 & -0.5 & 2.28 & 2.26 & -0.9 \\
\hline$\hat{V}_{4}^{i m}$ & 2.12 & 2.10 & -0.9 & 2.08 & 2.07 & -0.5 & 2.23 & 2.17 & -2.7 \\
\hline$\hat{V}_{5}^{i m}$ & 2.12 & 2.10 & -0.9 & 2.08 & 2.08 & 0.0 & 2.22 & 2.18 & -1.8 \\
\hline$\hat{V}_{6}^{i m}$ & 2.17 & 2.15 & -0.9 & 2.13 & 2.12 & -0.5 & 2.23 & 2.25 & 0.9 \\
\hline$\hat{V}_{7}^{i m}$ & 2.14 & 2.11 & -1.4 & 2.09 & 2.09 & 0.0 & 2.23 & 2.19 & -1.8 \\
\hline$\hat{V}_{8}^{i m}$ & 2.28 & 2.22 & -2.6 & 2.20 & 2.19 & -0.5 & 2.36 & 2.36 & 0.0 \\
\hline$\hat{V}_{9}^{i m}$ & 2.15 & 2.12 & -1.4 & 2.09 & 2.09 & 0.0 & 2.24 & 2.20 & -1.8 \\
\hline$\hat{V}_{10}^{i m}$ & 2.19 & 2.15 & -1.8 & 2.12 & 2.12 & 0.0 & 2.28 & 2.25 & -1.3 \\
\hline$\hat{V}_{11}^{i m}$ & 2.38 & 2.31 & -2.9 & 2.30 & 2.28 & -0.9 & 2.53 & 2.51 & -0.8 \\
\hline$\hat{V}_{12}^{i m}$ & 2.32 & 2.29 & -1.3 & 2.27 & 2.26 & -0.4 & 2.47 & 2.47 & 0.0 \\
\hline$\hat{V}_{13}^{i m}$ & 2.19 & 2.16 & -1.4 & 2.14 & 2.13 & -0.5 & 2.24 & 2.27 & 1.3 \\
\hline$\hat{V}_{14}^{i m}$ & 2.31 & 2.28 & -1.3 & 2.24 & 2.24 & 0.0 & 2.42 & 2.45 & 1.2 \\
\hline
\end{tabular}

Variances unit: $\times 10^{-6}$;

$\%$ Error $=($ Eq. $(3.20)-$ Simulation $) /$ Simulation $\times 100 \%$. 
in Fig. 3.5 shows that Sensor 3 with standard deviation $\sigma_{3} \leq 3 \times 10^{-3}$ should be selected. The crosses in both figures are variances obtained from simulations and they are well approximated by the solid-line drawn with (3.20).

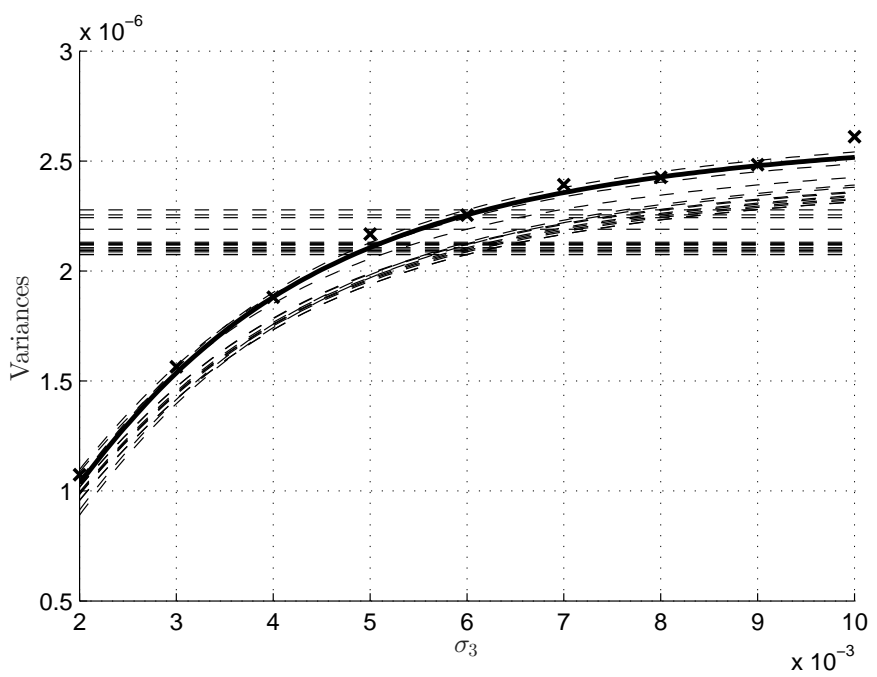

Figure 3.5: Variances of all the 28 state estimates versus $\sigma_{3}$. The solid-line is the variance of state estimate $\hat{V}_{12}^{r}$. The crosses are the variances of state estimate $\hat{V}_{12}^{r}$ obtained from simulation.

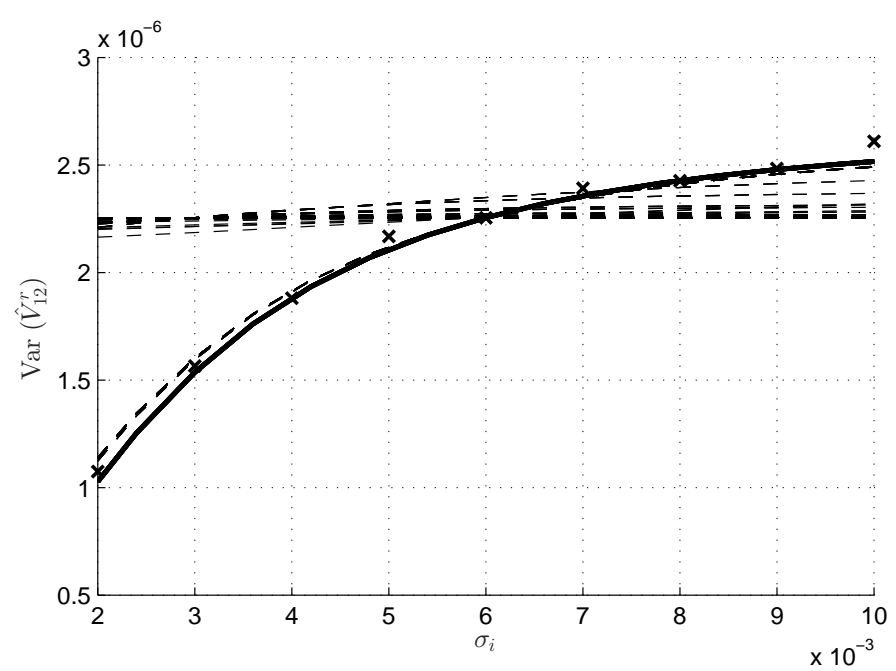

Figure 3.6: Variance of state estimate $\hat{V}_{12}^{r}$ versus $\sigma_{i}, i=1, \ldots, 58$. The solid-line shows the variance of state estimate $\hat{V}_{12}^{r}$ versus $\sigma_{3}$. The crosses are the variances of state estimate $\hat{V}_{12}^{r}$ versus $\sigma_{3}$ obtained from simulation. 
Chapter 3. Variance Analysis of Robust State Estimation in Power Systems using Influence Function ${ }^{1}$

\subsubsection{Example 3: Optimal Estimator Design}

In this example it is wished to find the optimum parameters $a_{i}, b_{i}$ and $r_{i}$ of the MS estimator to give the minimum sum of the state estimate variances defined as

$$
\mathrm{SV}=\sum_{i=1}^{28} \operatorname{Var}\left(\hat{x}_{i}\right)
$$

for more precise estimation results.

Consider the same IEEE 14-bus system with PMU measurements in Example 2 but let the noise $\epsilon_{i}$ be associated with the following $t$-distribution which is commonly used to model noise with outliers [39]

$$
f_{i}\left(\epsilon_{i}\right)=\frac{\Gamma\left(\frac{\xi_{i}+1}{2}\right)}{\sqrt{\xi_{i} \pi}\left(\sigma_{i}^{t}\right) \Gamma\left(\frac{\xi_{i}}{2}\right)}\left(1+\frac{\left|\epsilon_{i}\right|^{2}}{\left(\sigma_{i}^{t}\right)^{2} \xi_{i}}\right)^{-\frac{\xi_{i}+1}{2}}
$$

where $\Gamma(\cdot)$ is the Gamma function; $\sigma_{i}^{t}$ is the scale parameter; and $\xi_{i}$ is the shape parameter. In this example, the parameters for voltage measurements i.e. $i=$ $1, \ldots, 12$ are $\xi_{i}=3\left(t_{3}\right.$ distribution) and $\sigma_{i}^{t}=0.006$. And the ones for current measurements i.e. $i=13, \ldots, 58$ are $\xi_{i}=5\left(t_{5}\right.$ distribution $)$ and $\sigma_{i}^{t}=0.003$.

A search was conducted in the ranges $a_{i}=0.5,0.6, \ldots, 1.4, b_{i}=0.5,0.6, \ldots, 1.4$, $r_{i}=6,7, \ldots, 15$ to find the minimum SV in (3.37). Since each range of $a_{i}, b_{i}$ and $r_{i}$ consists of 10 points and 2 independent sets of $a_{i}, b_{i}$ and $r_{i}$ are chosen for the voltage measurements and current measurements, a total of $(10 \times 10 \times 10-450)^{2}=302,500$ combinations of points were searched. The substraction of 450 is to account for the constraint $a_{i} \leq b_{i} \leq r_{i}$. For each of the 302,500 combinations, (3.20) was used to calculate a set of 28 variances which were then added together according to (3.37) to give SV. The search then gave the minimum $\mathrm{SV}=87.51 \times 10^{-6}$ with $a_{i}=0.6, b_{i}=1$, $r_{i}=7$ for $i=1, \ldots, 12$ and $a_{i}=1, b_{i}=1.1, r_{i}=13$ for $i=13, \ldots, 58$ as shown in Table 3.4 (see Row "SV", Column "MS (optimal)-Eq. (3.20)"). In contrast, if 
typical values of $a_{i}=2.5, b_{i}=3.5, r_{i}=4.5$ for $i=1, \ldots, 12$ and $a_{i}=3, b_{i}=4, r_{i}=5$ for $i=13, \ldots, 58$ were chosen then $\mathrm{SV}=117.64 \times 10^{-6}$ as shown in Table 3.4 (see Row "SV", Column "MS (typical)-Eq. (3.20)"). The optimum parameters reduced the sum of variances by about $(117.64-87.51) \times 10^{-6} /(117.64) \times 10^{-6} \times 100=26 \%$. Hence the optimal estimator gives more precise state estimates.

Simulation can be used to obtain $\hat{x}$ but to determine variance, many data points and hence many simulation runs are required to achieve convergence. Ten thousand simulation runs using Matlab version R2015a on a $i 7$ Windows 10 computer with 8 GB RAM took 145 s to give a set of 28 variances. On the same equipment, equation (3.20) took less than 0.001s to perform the same task. Even if a lower level of precision can be accepted and the simulation runs are reduced to 1000, it will still take 14.5s. At this lower level of precision, going through 302,500 combinations requires $14.5 \mathrm{~s} \times 302,500 \approx 50$ days. In contrast, equation (3.20) takes $0.001 \mathrm{~s} \times 302,500$ $\approx 5$ minutes, making the optimal estimator design a more tractable problem. For simplicity exhaustive search is used. Independent of the search method, Equation $(3.20)$ is $14,500(=14.5 / 0.001)$ times faster than Monte Carlo simulation.

To put things in perspective, a well-known optimal estimator, the maximum likelihood estimator was also designed for the given IEEE 14-bus system and its IF plotted in Fig. 3.7. The IF of the MS (optimal) and MS (typical) estimators given in Table 3.4 are superimposed onto the figure. It is clear from the figure that the IF of the MS (optimal) estimator is close to optimum i.e. the maximum likelihood while the MS (typical) estimator is not. Note that the IF is a function of $\epsilon_{i} i=1, \ldots, 58$ but for simplicity, the IF curve is plotted assuming $\epsilon_{1}=\epsilon_{2}=\ldots=\epsilon_{58}$. 
Chapter 3. Variance Analysis of Robust State Estimation in Power Systems using Influence Function ${ }^{1}$

Table 3.4: Variances of the state estimates of the MS (optimal) and MS (typical) estimators in the IEEE 14-bus system.

\begin{tabular}{|c|c|c|c|c|c|c|}
\hline & \multicolumn{3}{|c|}{$\begin{array}{l}\quad \text { MS (typical) } \\
a_{i}=2.5, b_{i}=3.5, r_{i}=4.5, \\
\text { for } i=1, \ldots, 12 ; \\
a_{i}=3, b_{i}=4, r_{i}=5, \\
\text { for } i=13, \ldots, 58 .\end{array}$} & \multicolumn{3}{|c|}{$\begin{array}{l}\quad \text { MS (optimal) } \\
a_{i}=0.6, b_{i}=1, r_{i}=7, \\
\text { for } i=1, \ldots, 12 ; \\
a_{i}=1, b_{i}=1.1, r_{i}=13, \\
\text { for } i=13, \ldots, 58 .\end{array}$} \\
\hline & $\begin{array}{l}\text { Simu } \\
\text { lation }\end{array}$ & $\begin{array}{c}\text { Eq. } \\
(3.20)\end{array}$ & $\begin{array}{c}\% \\
\text { Error }\end{array}$ & $\begin{array}{l}\text { Simu } \\
\text { lation }\end{array}$ & $\begin{array}{c}\text { Eq. } \\
(3.20)\end{array}$ & $\begin{array}{c}\% \\
\text { Error }\end{array}$ \\
\hline$\hat{V}_{1}^{r}$ & 4.23 & 4.15 & -1.8 & 3.15 & 3.08 & -2.4 \\
\hline$\hat{V}_{2}^{r}$ & 4.23 & 4.14 & -1.9 & 3.14 & 3.07 & -2.2 \\
\hline$\hat{V}_{3}^{r}$ & 4.28 & 4.17 & -2.6 & 3.17 & 3.10 & -2.2 \\
\hline$\hat{V}_{4}^{r}$ & 4.20 & 4.11 & -2.2 & 3.11 & 3.04 & -2.2 \\
\hline$\hat{V}_{5}^{r}$ & 4.19 & 4.11 & -2.0 & 3.11 & 3.04 & -2.1 \\
\hline$\hat{V}_{6}^{r}$ & 4.27 & 4.17 & -2.3 & 3.18 & 3.10 & -2.7 \\
\hline$\hat{V}_{7}^{r}$ & 4.21 & 4.13 & -2.0 & 3.13 & 3.06 & -2.4 \\
\hline$\hat{V}_{8}^{r}$ & 4.35 & 4.27 & -2.0 & 3.29 & 3.18 & -3.1 \\
\hline$\hat{V}_{9}^{r}$ & 4.22 & 4.13 & -2.0 & 3.15 & 3.07 & -2.6 \\
\hline$\hat{V}_{10}^{r}$ & 4.27 & 4.17 & -2.3 & 3.18 & 3.10 & -2.4 \\
\hline$\hat{V}_{11}^{r}$ & 4.52 & 4.39 & -2.8 & 3.42 & 3.29 & -3.7 \\
\hline$\hat{V}_{12}^{r}$ & 4.47 & 4.36 & -2.5 & 3.34 & 3.26 & -2.4 \\
\hline$\hat{V}_{13}^{r}$ & 4.28 & 4.19 & -2.3 & 3.20 & 3.11 & -2.9 \\
\hline$\hat{V}_{14}^{r}$ & 4.41 & 4.34 & -1.6 & 3.35 & 3.25 & -3.1 \\
\hline$\hat{V}_{1}^{i m}$ & 4.20 & 4.15 & -1.3 & 3.20 & 3.08 & -3.9 \\
\hline$\hat{V}_{2}^{i m}$ & 4.19 & 4.14 & -1.1 & 3.20 & 3.07 & -3.8 \\
\hline$\hat{V}_{3}^{i m}$ & 4.20 & 4.17 & -0.7 & 3.22 & 3.10 & -3.7 \\
\hline$\hat{V}_{4}^{i m}$ & 4.15 & 4.11 & -0.9 & 3.15 & 3.04 & -3.5 \\
\hline$\hat{V}_{5}^{i m}$ & 4.15 & 4.11 & -0.9 & 3.15 & 3.04 & -3.5 \\
\hline$\hat{V}_{6}^{i m}$ & 4.19 & 4.17 & -0.5 & 3.22 & 3.10 & -3.9 \\
\hline$\hat{V}_{7}^{i m}$ & 4.15 & 4.13 & -0.6 & 3.18 & 3.06 & -3.9 \\
\hline$\hat{V}_{8}^{i m}$ & 4.30 & 4.27 & -0.6 & 3.33 & 3.18 & -4.5 \\
\hline$\hat{V}_{9}^{i m}$ & 4.15 & 4.13 & -0.5 & 3.18 & 3.07 & -3.7 \\
\hline$\hat{V}_{10}^{i m}$ & 4.19 & 4.17 & -0.6 & 3.22 & 3.10 & -3.7 \\
\hline$\hat{V}_{11}^{i m}$ & 4.43 & 4.39 & -1.0 & 3.44 & 3.29 & -4.3 \\
\hline$\hat{V}_{12}^{i m}$ & 4.40 & 4.36 & -1.0 & 3.42 & 3.26 & -4.5 \\
\hline$\hat{V}_{13}^{i m}$ & 4.19 & 4.19 & -0.2 & 3.23 & 3.11 & -3.8 \\
\hline$\hat{V}_{14}^{i m}$ & 4.37 & 4.34 & -0.6 & 3.38 & 3.25 & -4.0 \\
\hline SV & 119.39 & 117.64 & -1.5 & 90.45 & 87.51 & -3.3 \\
\hline
\end{tabular}

Variances unit: $\times 10^{-6}$;

$\%$ Error $=($ Eq. $(3.20)-$ Simulation $) /$ Simulation $\times 100 \%$. 


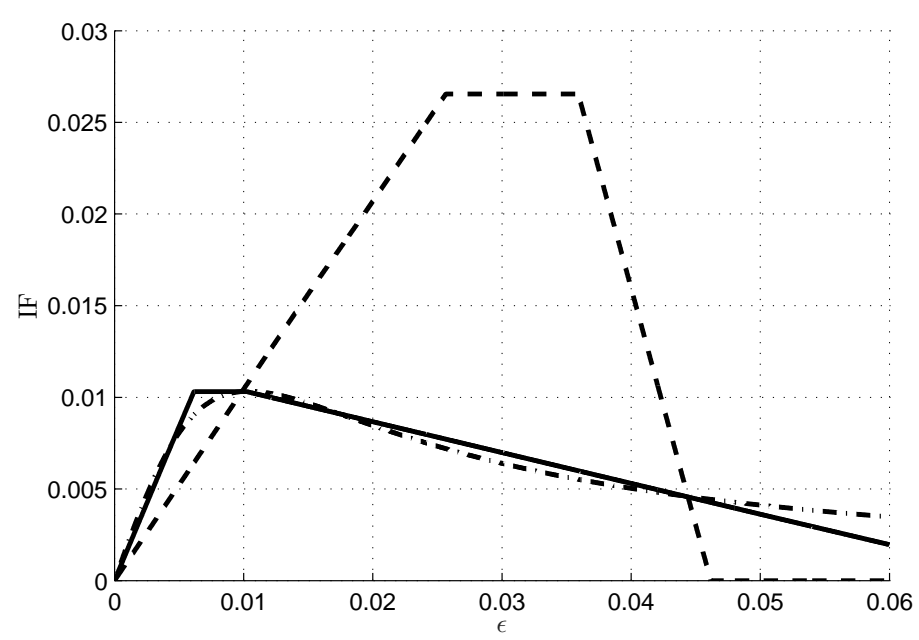

Figure 3.7: IF of maximum likelihood estimator: dash-dotted-line; MS (optimal) estimator: solid-line; MS (typical) estimator: dash-line.

\subsubsection{Example 4: The IEEE 30-bus System with PMU mea- surements}

To further verify variance equation (3.20), MS state estimation of a larger system, the IEEE 30-bus system, is simulated in this example. Some relevant parameters are given in Table 3.5 .

The PMUs are placed according to [4], as shown in Fig. 3.8. Since PMUs are used, the linear measurement model in (3.1) is applicable. The matrix $H$ is chosen according to the parameters in [92]. There are $n=60$ states in the state vector $x=$ $\left[V_{1}^{r} V_{2}^{r} \cdots V_{30}^{r} V_{1}^{i m} \cdots V_{30}^{i m}\right]^{T}$. Eighty-six measurements, $z_{i}, i=1, \ldots, 86$, comprising of 28 voltages $(i=1, \ldots, 28)$ and 58 currents $(i=29, \ldots, 86)$ are taken at each time instance $k$ and $N=3$ sets of measurements i.e. $k=1,2,3$ are used to obtain the estimated results.

For the voltage measurements $z_{i}, i=1, \ldots, 28$, the parameters of the MS estimators are chosen as $a_{i}=2.5, b_{i}=3.5, r_{i}=4.5$ [31]. The noise $\epsilon_{i}$ is associated with the pdf (3.34) where $\sigma_{i}=0.006, i=1, \ldots 28$ [93].

For the current measurements $z_{i}, i=29, \ldots, 86$, the parameters for the MS esti- 
Chapter 3. Variance Analysis of Robust State Estimation in Power Systems using Influence Function ${ }^{1}$

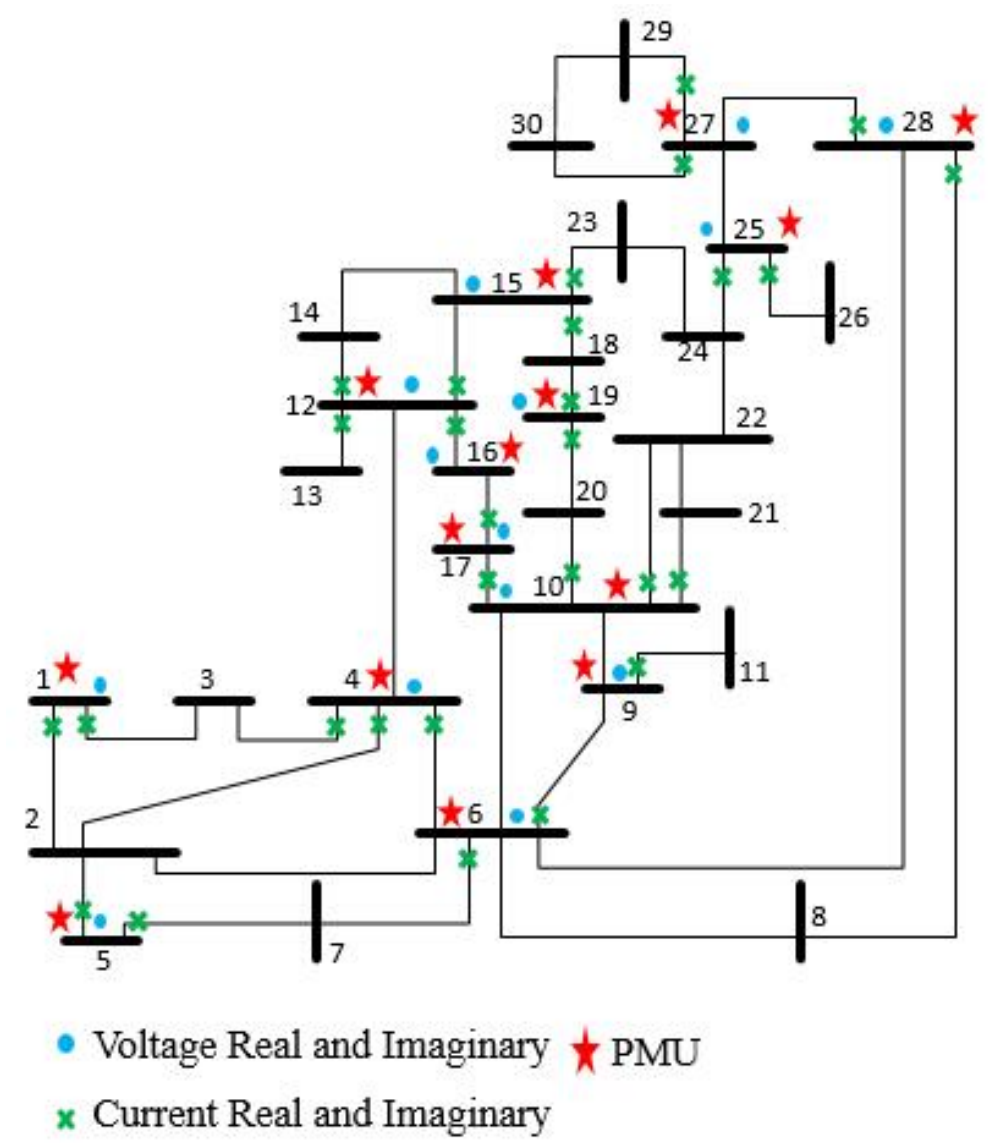

Figure 3.8: The IEEE 30-bus system installed with PMUs [4]. 
Table 3.5: Parameters of estimators and noises used in the IEEE 30-bus system.

\begin{tabular}{|c|c|c|}
\hline Example & \multicolumn{2}{|c|}{4} \\
\hline Model & \multicolumn{2}{|c|}{$\begin{array}{c}\text { IEEE 30-bus System } \\
\text { with PMU Measurements }\end{array}$} \\
\hline $\begin{array}{c}\text { Measure- } \\
\text { ment } i\end{array}$ & $1, \ldots, 28$ & $29, \ldots, 86$ \\
\hline $\begin{array}{c}\text { Normal } \\
\text { Noise }\end{array}$ & $\begin{array}{c}0.99 \times \\
{ }^{2} N(0.006)\end{array}$ & $\begin{array}{c}0.97 \times \\
N(0.003)\end{array}$ \\
\hline Outliers & $0.01 N(0.06)$ & $0.03 U(0.03)$ \\
\hline \multirow{2}{*}{ Estimator } & $\mathrm{MS}(2.5,3.5,4.5)$ & $\mathrm{MS}(3,4,5)$ \\
\hline Table & \multicolumn{2}{|c|}{3.6} \\
\hline
\end{tabular}

${ }^{*} N\left(\sigma_{i}\right)=\frac{1}{\sqrt{2 \pi \sigma_{i}^{2}}} e^{-\frac{\epsilon_{i}^{2}}{2 \sigma_{i}^{2}}}$.

${ }^{\dagger} U\left(\sigma_{i}\right)=\frac{1}{2 \sigma_{i}}$ for $-\sigma_{i} \leq \epsilon_{i} \leq \sigma_{i}$.

$\ddagger$ Parameters $a_{i}, b_{i}, r_{i}$ are given in the bracket of $\operatorname{MS}\left(a_{i}, b_{i}, r_{i}\right)$.

mator are chosen as $a_{i}=3, b_{i}=4, r_{i}=5$. The noise $\epsilon_{i}$ is associated with pdf (3.35) where $\sigma_{i}=0.003, i=29, \ldots, 86[93]$.

Since the detail calculations are already illustrated through a simple system in Example 1, only the results of the calculations are given in Table 3.6.

The variances calculated from (3.20) are given in Table 3.6 under column "Eq. (3.20)". To give an idea of the accuracy, the variance calculated from (3.20) is compared with the variance obtained from 10,000 simulation runs and the results are also given in Table 3.6. The maximum percentage error between the variances from simulation and from (3.20) given under column "\% Error" is less than 5\% which in practice should be good enough. 
Chapter 3. Variance Analysis of Robust State Estimation in Power Systems using Influence Function ${ }^{1}$

Table 3.6: Variances of the state estimates from the MS estimator for the IEEE 30-bus system with PMU measurements.

\begin{tabular}{|c|c|c|c|c|c|c|c|}
\hline \multicolumn{8}{|c|}{$\begin{array}{l}\mathrm{MS} \\
a_{i}=2.5, b_{i}=3.5, r_{i}=4.5, \text { for } i=1, \ldots, 28 \\
a_{i}=3, b_{i}=4, r_{i}=5, \text { for } i=29, \ldots, 86\end{array}$} \\
\hline States & $\begin{array}{l}\text { Simu } \\
\text { lation }\end{array}$ & $\begin{array}{c}\text { Eq. } \\
(3.20)\end{array}$ & $\begin{array}{c}\% \\
\text { Error }\end{array}$ & States & $\begin{array}{l}\text { Simu } \\
\text { lation }\end{array}$ & $\begin{array}{c}\text { Eq. } \\
(3.20)\end{array}$ & $\begin{array}{c}\% \\
\text { Error }\end{array}$ \\
\hline$\hat{V}_{1}^{r}$ & 2.53 & 2.49 & -1.4 & $\hat{V}_{1}^{i m}$ & 2.54 & 2.49 & $\begin{array}{l}-2.1 \\
\end{array}$ \\
\hline$\hat{V}_{2}^{r}$ & 2.52 & 2.48 & -1.5 & $\hat{V}_{2}^{i m}$ & 2.53 & 2.48 & -2.1 \\
\hline$\hat{V}_{3}^{r}$ & 2.50 & 2.47 & -1.2 & $\hat{V}_{3}^{i m}$ & 2.52 & 2.47 & -2.1 \\
\hline$\hat{V}_{4}^{r}$ & 2.50 & 2.47 & -1.2 & $\hat{V}_{4}^{i m}$ & 2.52 & 2.47 & -2.0 \\
\hline$\hat{V}_{5}^{r}$ & 2.54 & 2.50 & -1.5 & $\hat{V}_{5}^{i m}$ & 2.55 & 2.50 & -2.1 \\
\hline$\hat{V}_{6}^{r}$ & 2.50 & 2.47 & -1.2 & $\hat{V}_{6}^{i m}$ & 2.51 & 2.47 & -1.9 \\
\hline$\hat{V}_{7}^{r}$ & 2.51 & 2.48 & -1.4 & $\hat{V}_{7}^{i m}$ & 2.53 & 2.48 & -2.1 \\
\hline$\hat{V}_{8}^{r}$ & 6.46 & 6.36 & -1.5 & $\hat{V}_{8}^{i m}$ & 6.39 & 6.36 & -0.5 \\
\hline$\hat{V}_{9}^{r}$ & 2.58 & 2.55 & -1.1 & $\hat{V}_{9}^{i m}$ & 2.59 & 2.55 & -1.5 \\
\hline$\hat{V}_{10}^{r}$ & 2.13 & 2.10 & -1.3 & $\hat{V}_{10}^{i m}$ & 2.10 & 2.10 & 0.1 \\
\hline$\hat{V}_{11}^{r}$ & 2.74 & 2.69 & -1.6 & $\hat{V}_{11}^{i m}$ & 2.73 & 2.69 & -1.3 \\
\hline$\hat{V}_{12}^{r}$ & 2.12 & 2.11 & -0.5 & $\hat{V}_{12}^{i m}$ & 2.09 & 2.11 & 1.2 \\
\hline$\hat{V}_{13}^{r}$ & 2.20 & 2.18 & -0.8 & $\hat{V}_{13}^{i m}$ & 2.15 & 2.18 & 1.1 \\
\hline$\hat{V}_{14}^{r}$ & 2.41 & 2.38 & -1.2 & $\hat{V}_{14}^{i m}$ & 2.37 & 2.38 & 0.4 \\
\hline$\hat{V}_{15}^{r}$ & 2.13 & 2.12 & -0.5 & $\hat{V}_{15}^{i m}$ & 2.09 & 2.12 & 1.4 \\
\hline$\hat{V}_{16}^{r}$ & 2.12 & 2.11 & -0.8 & $\hat{V}_{16}^{i m}$ & 2.08 & 2.11 & 1.4 \\
\hline$\hat{V}_{17}^{r}$ & 2.13 & 2.10 & -1.3 & $\hat{V}_{17}^{i m}$ & 2.10 & 2.10 & 0.1 \\
\hline$\hat{V}_{18}^{r}$ & 2.18 & 2.15 & -1.4 & $\hat{V}_{18}^{i m}$ & 2.13 & 2.15 & 0.6 \\
\hline$\hat{V}_{19}^{r}$ & 2.16 & 2.13 & -1.2 & $\hat{V}_{19}^{i m}$ & 2.12 & 2.13 & 0.7 \\
\hline$\hat{V}_{20}^{r}$ & 2.16 & 2.13 & -1.3 & $\hat{V}_{20}^{i m}$ & 2.13 & 2.13 & 0.4 \\
\hline$\hat{V}_{21}^{r}$ & 2.16 & 2.12 & -1.5 & $\hat{V}_{21}^{i m}$ & 2.13 & 2.12 & -0.1 \\
\hline$\hat{V}_{22}^{r}$ & 2.23 & 2.19 & -1.5 & $\hat{V}_{22}^{i m}$ & 2.21 & 2.19 & -0.7 \\
\hline$\hat{V}_{23}^{r}$ & 2.31 & 2.29 & -1.0 & $\hat{V}_{23}^{i m}$ & 2.28 & 2.29 & 0.5 \\
\hline$\hat{V}_{24}^{r}$ & 13.2 & 12.8 & -3.0 & $\hat{V}_{24}^{i m}$ & 13.2 & 12.8 & -3.5 \\
\hline$\hat{V}_{25}^{r}$ & 12.6 & 12.3 & -2.6 & $\hat{V}_{25}^{i m}$ & 12.7 & 12.3 & -3.1 \\
\hline$\hat{V}_{26}^{r}$ & 13.3 & 13.0 & -2.1 & $\hat{V}_{26}^{i m}$ & 13.5 & 13.0 & -3.6 \\
\hline$\hat{V}_{27}^{r}$ & 6.34 & 6.26 & -1.2 & $\hat{V}_{27}^{i m}$ & 6.30 & 6.26 & -0.6 \\
\hline$\hat{V}_{28}^{r}$ & 6.37 & 6.26 & -1.7 & $\hat{V}_{28}^{i m}$ & 6.31 & 6.26 & -0.7 \\
\hline$\hat{V}_{29}^{r}$ & 7.14 & 6.99 & -2.1 & $\hat{V}_{29}^{i m}$ & 7.07 & 6.99 & -1.1 \\
\hline$\hat{V}_{30}^{r}$ & 8.07 & 7.80 & -3.4 & $\hat{V}_{30}^{i m}$ & 7.97 & 7.80 & -2.2 \\
\hline
\end{tabular}

Variances unit: $\times 10^{-6}$;

$\%$ Error $=($ Eq. $(3.20)-$ Simulation $) /$ Simulation $\times 100 \%$. 


\subsection{Summary}

In this chapter an analytical equation is derived using influence function approximation to calculate the variance of the state estimate approximately for common robust state estimators such as the Quadratic-Constant, Quadratic-Linear, SquareRoot, Multiple-Segment and Schweppe-Huber Generalized-M estimator. The wellknown WLS variance formula is a special case of the equation. The derived equation can also be used to: (1) design an optimal state estimator. Supposed the PMUs with different precision grades have been installed in some power system, but the precision grades (or the standard deviations ) of PMUs are known. It is clear that we can follow the approach described in Section 3.4.3 and find the optimal estimator to this specific power system. and (2) express the variance of a state estimate as a function of measurement variances enabling the selection of sensors for the specified estimator precision. In a real power system, if we find the variance of some state is much bigger than that of others, It is easy for us to follow the method provided in Example 2, and then we can show the relationship between the variance of some state and the standard deviation of some sensor. Finally we can choose a suitable sensor to reduce the variance of the specific state. 


\section{Chapter 4}

\section{Robust Power System State}

\section{Estimation Using $t$-Distribution}

\section{Noise Model $^{2}$}

In this chapter, a robust estimator based on the maximum likelihood criterion is proposed for robust PSSE, where the $t$-distribution probability density function is used to fit the noise so as to get a noise model with higher accuracy. The $t$ distribution has the property of "thick tail" to better model the occurrence of outliers $[39,94,95]$. Hence the $t$-distribution has the flexibility to characterize noise with different statistical properties. The tail property to model the noise makes the state estimation less sensitive to the influence of outliers. The weights of the measurement errors are less than that of the WLS under the assumption of Gaussian distribution [96]. Instead of solving the optimization problem numerically, the Influence Function (IF) is employed to give an approximate solution to the problem. The proposed estimator can be readily implemented on existing hardware and software used in the

\footnotetext{
${ }^{2}$ Chapter 4 is based on our submitted paper "Tengpeng Chen, et al., Robust Power System State Estimation Using $t$-Distribution Noise Model. IEEE Transactions on Smart Grid, under review, 2017."
} 
robust estimator. The performance of the proposed robust estimator is compared with traditional estimators such as the QL, SR and MS estimator.

\subsection{Existing Robust State Estimation Algorithms}

For easy reference, equation (3.12) of the robust state estimation presented in Chapter 3 is repeated:

$$
\hat{x}=\left(\bar{H}^{T} W \bar{H}\right)^{-1} \bar{H}^{T} W Z
$$

where

$$
\begin{aligned}
\bar{H} & =\left[H^{T} \cdots H^{T}\right]^{T} \in \mathbb{R}^{N m \times n} \\
Z & =\left[z_{1}^{T} \cdots z_{N}^{T}\right]^{T} \in \mathbb{R}^{N m} \\
E & =\left[e_{1}^{T} \cdots e_{N}^{T}\right]^{T} \in \mathbb{R}^{N m} \\
W & =\operatorname{diag}\left(W_{1,1}, \ldots, W_{m, 1}, \ldots, W_{1, N}, \ldots, W_{m, N}\right) \in \mathbb{R}^{N m \times N m}
\end{aligned}
$$

$W_{i, k}$ for the MS and QL estimator is given in (3.13) as

$$
W_{i, k}= \begin{cases}\frac{1}{\sigma_{i}^{2}} & \left|e_{i, k}\right| \leq a_{i} \sigma_{i} \\ \frac{a_{i}}{\sigma_{i}\left|e_{i, k}\right|} & a_{i} \sigma_{i}<\left|e_{i, k}\right| \leq b_{i} \sigma_{i} \\ \frac{a_{i}\left(r_{i} \sigma_{i}-\left|e_{i, k}\right|\right)}{\left(r_{i}-b_{i}\right) \sigma_{i}^{2}\left|e_{i, k}\right|} & b_{i} \sigma_{i}<\left|e_{i, k}\right| \leq r_{i} \sigma_{i} \\ 0 & r_{i} \sigma_{i}<\left|e_{i, k}\right|\end{cases}
$$

$W_{i, k}$ for the SR estimator is given in (3.14) as

$$
W_{i, k}= \begin{cases}\frac{1}{\sigma_{i}^{2}} & \left|e_{i, k}\right| \leq a_{i} \sigma_{i} \\ \sqrt{\frac{a_{i}^{3}}{\sigma_{i}\left|e_{i, k}\right|^{3}}} & \text { otherwise }\end{cases}
$$


Chapter 4. Robust Power System State Estimation Using $t$-Distribution Noise Model $^{2}$

Using (4.2) or (4.3) to update $W$, and (4.1) is solved iteratively until the difference between the current and previous iteration for $\hat{x}$ is less than a specified tolerance.

\subsection{The Proposed Robust Estimator}

In this section, a new robust estimator based on maximum likelihood criterion is proposed, where $t$-distribution is used to model the distribution of the measurement noise and outliers. Instead of solving the optimization problem numerically, the IF is employed to give an approximate solution to the problem. The proposed estimator can be readily implemented on existing hardware and software used in the robust estimator.

\subsection{1 $\quad t$ Distribution}

Let the noise $\epsilon_{i}$ be modeled by the zero-mean $t$-distribution with pdf [39]

$$
f_{i}\left(\epsilon_{i}\right)=\frac{\Gamma\left(\frac{\xi_{i}+1}{2}\right)}{\sqrt{\xi_{i} \pi}\left(\sigma_{i}^{t}\right) \Gamma\left(\frac{\xi_{i}}{2}\right)}\left(1+\frac{\left|\epsilon_{i}\right|^{2}}{\left(\sigma_{i}^{t}\right)^{2} \xi_{i}}\right)^{-\frac{\xi_{i}+1}{2}}
$$

where $\Gamma(\cdot)$ is the Gamma function, $\sigma_{i}^{t}$ is the scale parameter, and $\xi_{i}$ is the shape parameter. The $t$ distributions with $\sigma_{i}^{t}=0.01$ and different $\xi_{i}$ are shown in Fig. 4.1, where the shape parameter increases. The $t$ distribution is symmetric like the normal distribution but it has heavier tails, which means that it is more prone to producing values that fall far from its mean. When the shape parameter $\xi_{i}$ tends to $\infty$, the $t$ distribution reduce to the Gaussian distribution 4.12 with $\sigma_{i}=0.01$. 


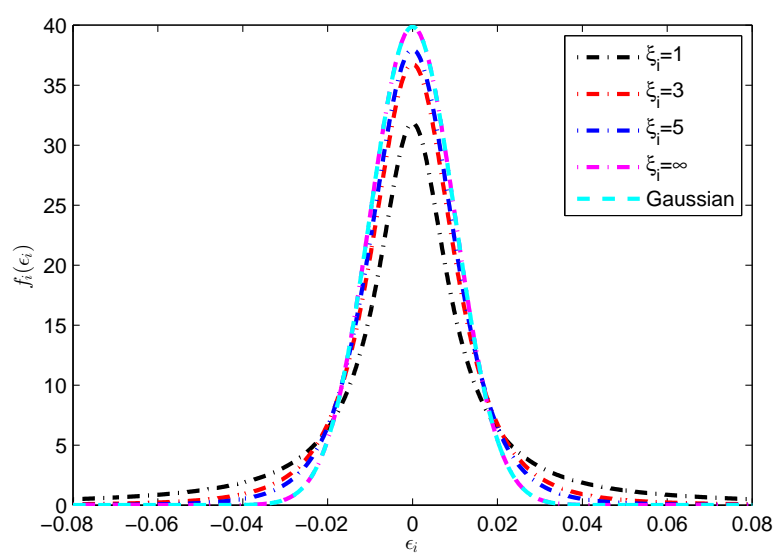

Figure 4.1: The $t$ distribution with different shape parameters.

\subsubsection{Maximum Likelihood Estimation}

In order to implement maximum likelihood estimation, it is necessary to find $\epsilon$ that maximizes the likelihood. It is equivalent to minimize:

$$
J_{o}=-\sum_{i=1}^{m} \sum_{k=1}^{N} \ln f_{i}\left(\epsilon_{i, k}\right)
$$

To minimize $J_{o}$, take the derivative of the $\log$ likelihood $J_{o}$ and use $\frac{\partial \epsilon_{i, k}}{\partial x}=-H_{i}^{T}$ :

$$
\begin{aligned}
\Psi_{o}(\varepsilon)=\frac{\partial J_{o}}{\partial x} & =-\sum_{i=1}^{m} \sum_{k=1}^{N} \frac{\partial \ln f_{i}\left(\epsilon_{i, k}\right)}{\partial \epsilon_{i, k}} \frac{1}{\epsilon_{i, k}} \epsilon_{i, k} \frac{\partial \epsilon_{i, k}}{\partial x} \\
& =-\sum_{i=1}^{m} \sum_{k=1}^{N} W_{i} \epsilon_{i, k} H_{i}^{T} \\
& =-\sum_{i=1}^{m} \sum_{k=1}^{N} \frac{\left(\xi_{i}+1\right) \epsilon_{i, k}}{\left(\sigma_{i}^{t}\right)^{2} \xi_{i}+\left(\epsilon_{i, k}\right)^{2}} H_{i}^{T}
\end{aligned}
$$

where $\varepsilon=\left[\epsilon_{1,1}, \cdots, \epsilon_{m, 1}, \cdots, \epsilon_{1, N} \cdots \epsilon_{m, N}\right]^{T}$ and $W_{i}=\frac{\xi_{i}+1}{\left(\sigma_{i}^{t}\right)^{2} \xi_{i}+\left(\epsilon_{i, k}\right)^{2}}$. 
Chapter 4. Robust Power System State Estimation Using $t$-Distribution Noise Model $^{2}$

\subsubsection{Influence Function Approximation}

The IF for MLE is given as

$$
\operatorname{IF}(\varepsilon)=-\left[\int_{-\infty}^{\infty} \frac{\partial \Psi_{o}(\varepsilon)}{\partial x} d F(\varepsilon)\right]^{-1} \Psi_{o}(\varepsilon) \approx \hat{x}-x
$$

where $d F(\varepsilon)$ is given by

$$
\begin{aligned}
d F(\varepsilon)= & f_{1}\left(\epsilon_{1,1}\right) \times f_{2}\left(\epsilon_{2,1}\right) \times \cdots \times f_{m}\left(\epsilon_{m, 1}\right) \times \cdots \times f_{1}\left(\epsilon_{1, N}\right) \times f_{2}\left(\epsilon_{2, N}\right) \times \cdots \times f_{m}\left(\epsilon_{m, N}\right) \\
& \times d \epsilon_{1,1} \times d \epsilon_{2,1} \times \cdots \times d \epsilon_{m, 1} \times \cdots \times d \epsilon_{1, N} \times d \epsilon_{2, N} \times \cdots \times d \epsilon_{m, N} .
\end{aligned}
$$

since the measurement noise $\epsilon_{i}$ is assumed to be independent and identically distributed.

Using (4.7), $\hat{x}$ is given as:

$$
\hat{x} \approx x+\operatorname{IF}(\varepsilon)
$$

In practice, since $x$ is unknown, the noise vector $\varepsilon=Z-\bar{H} x$ is replaced by $E=$ $Z-\bar{H} \hat{x}$ and (4.8) is used to find $\hat{x}$ iteratively given an initial guess $\hat{x}$.

$$
\hat{x} \leftarrow \hat{x}+\operatorname{IF}(E)
$$

where

$$
\operatorname{IF}(E)=\left(\bar{H}^{T} \Omega \bar{H}\right)^{-1} \bar{H}^{T} \Lambda E
$$

The diagonal matrices $\Omega$ and $\Lambda$ can be calculated as follows: 
According to (4.6) and (4.7), $\varepsilon$ is replaced by $E$, and then $\operatorname{IF}(E)$ is given by

$$
\begin{aligned}
\operatorname{IF}(E) & =\left(\bar{H}^{T} \Omega \bar{H}\right)^{-1} \bar{H} \Lambda E \\
& =-\left[\int_{-\infty}^{\infty} \frac{\partial \Psi_{o}(\varepsilon)}{\partial x} d F(\varepsilon)\right]^{-1} \Psi_{o}(E) \\
& =-\left[\int_{-\infty}^{\infty} \frac{\partial\left(\bar{H}^{T} W \varepsilon\right)}{\partial \varepsilon} \frac{\partial \varepsilon}{\partial x} d F(\varepsilon)\right]^{-1} \bar{H}^{T} W E \\
& =\left(\bar{H}^{T} \int_{-\infty}^{\infty} \frac{\partial(W \varepsilon)}{\partial \varepsilon} d F(\varepsilon) \bar{H}\right)^{-1} \bar{H}^{T} W E
\end{aligned}
$$

where

$$
\begin{aligned}
\Omega & =\int_{-\infty}^{\infty} \frac{\partial(W \varepsilon)}{\partial \varepsilon} d F(\varepsilon) \\
& =\operatorname{diag}\left(\Omega_{1,1}, \ldots, \Omega_{m, 1}, \ldots, \Omega_{1, N}, \ldots, \Omega_{m, N}\right) \\
\Omega_{i, k} & =\int_{-\infty}^{\infty} \frac{\partial W_{i} \epsilon_{i}}{\partial \epsilon_{i}} f_{i}\left(\epsilon_{i}\right) d \epsilon_{i} \\
& =\int_{-\infty}^{+\infty} \frac{\left(\xi_{i}+1\right)\left(\left(\sigma_{i}^{t}\right)^{2} \xi_{i}+\epsilon_{i}^{2}\right)-2\left(\xi_{i}+1\right) \epsilon_{i}^{2}}{\left(\left(\sigma_{i}^{t}\right)^{2} \xi_{i}+\epsilon_{i}^{2}\right)^{2}} f_{i}\left(\epsilon_{i}\right) d \epsilon_{i} \\
& =\int_{-\infty}^{+\infty} \frac{\left(\xi_{i}+1\right)\left(\left(\sigma_{i}^{t}\right)^{2} \xi_{i}-\epsilon_{i}^{2}\right)}{\left(\left(\sigma_{i}^{t}\right)^{2} \xi_{i}+\epsilon_{i}^{2}\right)^{2}} f_{i}\left(\epsilon_{i}\right) d \epsilon_{i} \\
\Lambda & =W=\operatorname{diag}\left(\Lambda_{1,1}, \ldots, \Lambda_{m, 1}, \ldots, \Lambda_{1, N}, \ldots, \Lambda_{m, N}\right) \\
\Lambda_{i, k} & =W_{i, k}=\frac{\xi_{i}+1}{\left(\sigma_{i}^{t}\right)^{2} \xi_{i}+\left(e_{i, k}\right)^{2}}
\end{aligned}
$$

The $t$-distribution model for the noise can be obtained by analyzing the historical measurement data. The matrix $\left(\bar{H}^{T} \Omega \bar{H}\right)^{-1}$ can be precomputed,thus saving computational load in real-time applications. The proposed estimator can be implemented just like other traditional robust state estimators presented as pseudo-code in Section 3.2.4. To implement the proposed estimator, only two modifications in 
Chapter 4. Robust Power System State Estimation Using t-Distribution Noise Model $^{2}$

the pseudo-code are needed as follows:

- Step (2.b.iv) in Section 3.2.4 is replaced with 'Renew $\Omega_{i}$ and $\Lambda_{i}$ using (4.10) and (4.11) respectively'.

- Step (2.b.v) in Section 3.2.4 is replaced with 'Calculate $\hat{x}$ using (4.8)'.

As a summary, the estimation $\hat{x}$ of the proposed robust estimator can be obtained by

$$
\hat{x} \leftarrow \hat{x}+\left(\bar{H}^{T} \Omega \bar{H}\right)^{-1} \bar{H}^{T} \Lambda E
$$

until the difference between the current and previous iteration for $\hat{x}$ is less than a specified tolerance. The diagonal elements of $\Omega$ and $\Lambda$ are given in (4.10) and (4.11) respectively.

\subsubsection{The Weighted-Least-Squares Connection}

The proposed estimator will reduce to WLS when the noise $\epsilon_{i}$ is Gaussian:

$$
f_{i}\left(\epsilon_{i}\right)=\frac{1}{\sqrt{2 \pi \sigma_{i}^{2}}} \exp \left(-\frac{\epsilon_{i}^{2}}{2 \sigma_{i}^{2}}\right)
$$

It is well known that (4.4) reduces to (4.12) when $\xi_{i} \rightarrow \infty$ and then there exists the relationship $\sigma_{i}^{t}=\sigma_{i}[39]$. According to (4.10) and (4.11), we get

$$
\begin{aligned}
& \Lambda_{i}=\lim _{\xi_{i} \rightarrow \infty} \frac{\left(\xi_{i}+1\right)}{\left(\sigma_{i}^{t}\right)^{2} \xi_{i}+\left(e_{i, k}\right)^{2}}=\frac{1}{\left(\sigma_{i}^{t}\right)^{2}}=\frac{1}{\sigma_{i}^{2}} \\
& \Omega_{i}=\lim _{\xi_{i} \rightarrow \infty} \int_{-\infty}^{+\infty} \frac{\left(\xi_{i}+1\right)\left(\left(\sigma_{i}^{t}\right)^{2} \xi_{i}-e_{i}^{2}\right)}{\left(\left(\sigma_{i}^{t}\right)^{2} \xi_{i}+e_{i}^{2}\right)^{2}} f_{i}\left(e_{i}\right) d e_{i}=\frac{1}{\sigma_{i}^{2}}
\end{aligned}
$$


Then the estimated results $\hat{x}$ is given as

$$
\begin{aligned}
\hat{x} & \leftarrow \hat{x}+\left(\bar{H}^{T} \Omega \bar{H}\right)^{-1} \bar{H}^{T} \Lambda E \\
& =\left(\bar{H}^{T} W \bar{H}\right)^{-1} \bar{H}^{T} W \bar{H} \hat{x}+\left(\bar{H}^{T} W \bar{H}\right)^{-1} \bar{H}^{T} W E \\
& =(\bar{H} W \bar{H})^{-1} \bar{H} W Z
\end{aligned}
$$

which is totally the same as (3.12).

\subsection{Simulation and Experiment Results}

In this section, simulation results obtained from the IEEE 14-bus (as shown in Fig. 3.4) and 118-bus systems installed with all PMUs are given in Example 1. Experimental results obtained from the 13-bus microgrid at Nanyang Technological University is given in Example 2. For easy reference, the probability density functions of the "normal" noise and outliers, the $t$-distribution fit noise, the Gaussian distribution fit using least square (LS) criterion and maximum likelihood (ML) criterion, and the parameters of the robust state estimators are summarized in Table 4.1.

\subsubsection{Example 1: Simulations Based on Linear Measure- ment Model}

In this example, a standard IEEE 14-bus system model is firstly built in MATLAB/Simulink environment. This model is then rebuilt to be suitable for the realtime operation mode in RT-LAB environment in terms of the operating requirements of RT-LAB. Hence the model running in the RT-LAB is assumed to be a real power system $[98,99]$. Meanwhile, the proposed estimator is programmed on a remote computer simulating the control center. The computer is connected to RT-LAB via 
Table 4.1: Parameters of estimators and noises used in the simulation examples.

\begin{tabular}{|c|c|c|c|}
\hline $\begin{array}{c}\text { Examples } \\
\text { Normal } \\
\text { Noise }\end{array}$ & ${ }^{*} N(0.005)$ & & 2 \\
\cline { 1 - 2 } Outliers & ${ }^{\dagger} U(0.005)$ & ${ }^{\sharp} t_{3}(0.005)$ & $\begin{array}{c}|c| \\
\text { raw } \\
\text { measurements }\end{array}$ \\
\hline$t$ fit & $t_{4}(0.0065)$ & $t_{3}(0.005)$ & $t_{3}(0.00004)$ \\
\hline${ }^{* *} \mathrm{LS}$ fit & $N(0.005)$ & $N(0.006)$ & $N(0.00003)$ \\
\hline${ }^{\dagger}$ ML fit & $N(0.007)$ & $N(0.009)$ & $N(0.000045)$ \\
\hline${ }^{\ddagger}$ Estimator & \multicolumn{2}{|c|}{${ }^{2} \mathrm{LAV}, \mathrm{QL}(3), \mathrm{SR}(3), \mathrm{MS}(3,4,5)$, Our method } \\
\hline
\end{tabular}

${ }^{*} N\left(\sigma_{i}\right)=0.95 \times \frac{1}{\sqrt{2 \pi \sigma_{i}^{2}}} \exp \left(-\frac{\epsilon_{i}^{2}}{2 \sigma_{i}^{2}}\right)$.

${ }^{\sharp} t_{3}\left(\sigma_{i}^{t}\right)=\frac{\Gamma(2)}{\sqrt{3 \pi}\left(\sigma_{i}^{t}\right) \Gamma(1.5)}\left(1+\frac{\left|\epsilon_{i}\right|^{2}}{3\left(\sigma_{i}^{t}\right)^{2}}\right)^{-2}$.

${ }^{\dagger} U\left(\sigma_{i}\right)=0.05 \times \frac{1}{4 \sigma_{i}}$ for $2 \sigma_{i} \leq\left|\epsilon_{i}\right| \leq 6 \sigma_{i}$.

${ }^{* *}$ LS fit: Using least squares criterion to get the Gaussian distribution, where the square of the distances between the pdf curve and the peak of each bar is minimized, see Fig. 4.3 for illustration.

${ }^{\dagger}$ ML fit: Using maximum likelihood criterion to get the Gaussian distribution [97].

$\ddagger$ Parameter $a_{i}$ is given in the bracket of $\mathrm{QL}\left(a_{i}\right)$ and $\mathrm{SR}\left(a_{i}\right)$ while parameters $a_{i}, b_{i}, r_{i}$ are given in the bracket of $\operatorname{MS}\left(a_{i}, b_{i}, r_{i}\right)$.

${ }^{\mathcal{S}} \mathrm{LAV}$ is only for linear case. 
cable. The configuration of the simulation is shown in Fig. 4.2.

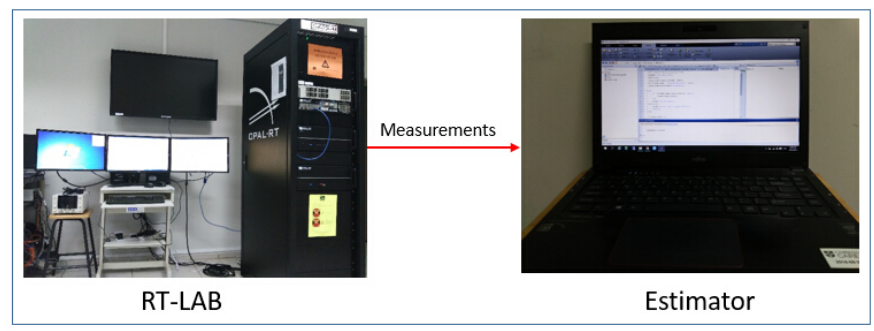

Figure 4.2: The configuration of the real time simulation.

For the IEEE 14-bus system, the PMUs are placed according to [91]. Fifty-eight measurements, $z_{i}, i=1, \ldots, 58$, comprising 12 voltages $(i=1, \ldots, 12)$ and 46 currents $(i=13, \ldots, 58)$ are taken at each time instance and $N=3$ sets of measurements are used to give one set of estimates. The measurement matrix $H$ is chosen according to the parameters in [92]. There are $n=28$ states in the vector $x=\left[V_{1}^{r} V_{2}^{r} \cdots V_{14}^{r} V_{1}^{i m} \cdots V_{14}^{i m}\right]^{T}$. The real and imaginary part of the voltage phasor are given as $V_{i}^{r}$ and $V_{i}^{i m}$ respectively. Two different types of measurement noise pdfs are considered in the simulation.

\section{Gaussian +1 Uniform $(1 \mathrm{G}+1 \mathrm{U})$}

The noise $\epsilon_{i}$ is associated with the pdf

$$
f_{i}\left(\epsilon_{i}\right)= \begin{cases}\frac{0.95}{\sqrt{2 \pi \sigma_{i}^{2}}} \exp \left(-\frac{\epsilon_{i}^{2}}{2 \sigma_{i}^{2}}\right)+\frac{0.05}{4 \sigma_{i}} & 2 \sigma_{i} \leq\left|\epsilon_{i}\right| \leq 6 \sigma_{i} \\ \frac{0.95}{\sqrt{2 \pi \sigma_{i}^{2}}} \exp \left(-\frac{\epsilon_{i}^{2}}{2 \sigma_{i}^{2}}\right) & \text { otherwise }\end{cases}
$$

where $\sigma_{i}=0.005$. The pdf in the form of a mixture distribution in (4.13) is also used in [32]. The uniform distribution in (4.13) is useful for modeling initial conditions, disturbances, and measurement errors that are equally likely to occur anywhere within a given interval [96]. Fig. 4.3 shows the fit results using different methods. The histogram represents the measurement noise generated by (4.13). The blue solid line is the fit result using $t$-distribution pdf $\xi_{i}=4$ ( $t_{4}$ distribution $), \sigma_{i}^{t}=0.0065$. 
Chapter 4. Robust Power System State Estimation Using t-Distribution Noise Model $^{2}$

According to $[97,100]$, the maximum likelihood criterion is also used to obtain the Gaussian distribution (ML fit) with $\sigma_{i}=0.007$. However, the ML fit does not visually match with the histogram well, as seen in Fig. 4.3. The least squares method is used to obtain a Gaussian pdf using the LS fit, with $\sigma_{i}=0.005$, where the distance of the pdf curve to the histogram bars are visually minimized. This dotted red curve fit the histogram well only when $\epsilon_{i}$ is within the range from -0.015 to +0.015 . As a summary, from Fig. 4.3 , it is clear that the $t$-distribution gives the best fit pdf while ML fit is worst. Thus the proposed estimator should perform better. In practical applications, the fit process can be done as a modelling step using historical measurements and it is calculated off-line.

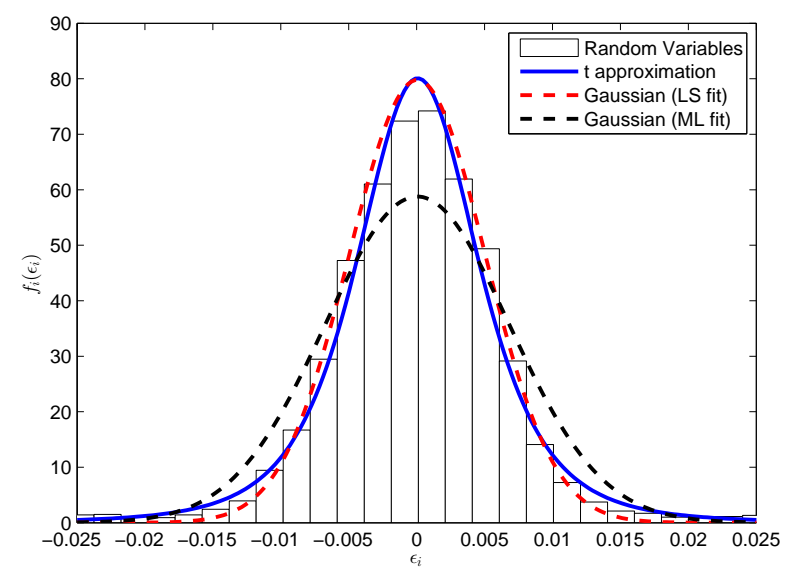

Figure 4.3: The $t$ distribution with $\xi_{i}=4, \sigma_{i}^{t}=0.0065$ and Gaussian distribution with $\sigma_{i}=0.005$ and $\sigma_{i}=0.007$ are used to fit the noise generalized according to (4.13) with $\sigma_{i}=0.005$.

The parameters for the QL and SR estimators are chosen as $a_{i}=3$ while the parameters for the MS estimator are $a_{i}=3, b_{i}=4, r_{i}=5$ according to [4]. The sum of variance, $\mathrm{SV}$,

$$
\mathrm{SV}=\sum_{i=1}^{n} \operatorname{Var}\left(\hat{x}_{i}\right)
$$

is used to evaluate the precision of estimation results obtained by different estimators. The variance results are given in Table 4.2. Take the QL estimator as an 
example, the SV using the least squares criterion is $69.9 \times 10^{-6}$ while the result is $78.3 \times 10^{-6}$ if the maximum likelihood criterion is used to fitted the Gaussian distribution. It is clear that the results using the LS fit is better. Due to the better approximation of the noise model, the proposed estimator is expected to provide better estimated results. The proposed estimator has an improvement of at least $12.2 \%$ to the SR estimator using the LS fit method and $16.7 \%$ to the LAV estimator in this case. In summary, the proposed robust estimator gets the minimum SV.

Table 4.2: Variances of the state estimates of the different estimators in the IEEE 14-bus system with PMU measurements.

\begin{tabular}{|c|c|c|c|c|c|c|c|c|c|c|}
\hline \multirow[t]{2}{*}{ Noise } & \multirow[t]{2}{*}{ Estimator } & \multirow[t]{2}{*}{ WLS } & \multicolumn{2}{|c|}{$\mathrm{QL}$} & \multicolumn{2}{|c|}{ SR } & \multicolumn{2}{|c|}{ MS } & \multirow[t]{2}{*}{ LAV } & \multirow{2}{*}{$\begin{array}{c}\text { Our } \\
\text { method }\end{array}$} \\
\hline & & & $\begin{array}{l}\text { LS } \\
\text { fit }\end{array}$ & $\begin{array}{l}\text { ML } \\
\text { fit }\end{array}$ & $\begin{array}{l}\text { LS } \\
\text { fit }\end{array}$ & $\begin{array}{c}\text { ML } \\
\text { fit }\end{array}$ & $\begin{array}{l}\text { LS } \\
\text { fit }\end{array}$ & $\begin{array}{c}\text { ML } \\
\text { fit }\end{array}$ & & \\
\hline \multirow[b]{2}{*}{$1 \mathrm{G}+1 \mathrm{U}$} & SV & 82.9 & 69.9 & 78.3 & 66.5 & 76.7 & 66.2 & 79.6 & 70.1 & 58.4 \\
\hline & $\begin{array}{c}\text { our method } \\
\text { improve- } \\
\text { ment }(\%)\end{array}$ & 29.6 & 16.5 & 25.4 & 12.2 & 23.9 & 11.8 & 26.6 & 16.7 & - \\
\hline \multirow[b]{2}{*}{$t_{3}$} & SV & 133.7 & 95.5 & 102.3 & 91.2 & 98.8 & 90.3 & 97.9 & 78.1 & 71.4 \\
\hline & $\begin{array}{l}\text { our method } \\
\text { improve- } \\
\operatorname{ment}(\%)\end{array}$ & 46.6 & 25.2 & 30.2 & 21.7 & 27.7 & 20.9 & 27.1 & 8.6 & - \\
\hline
\end{tabular}

Variances unit: $\times 10^{-6}$.

\section{$t_{3}$ Noise}

In this subsection, we firstly consider the same IEEE 14-bus system with PMU measurements but let the noise $\epsilon_{i}$ be associated with the following $t$-distribution which is commonly used to model noise with outliers [26]. The parameters in (4.4) are chosen as: $\xi_{i}=3, \sigma_{i}^{t}=0.005$ for measurement noises. The Gaussian approximation parameters are given in Table 4.1. The variance results are given in Table 4.2. Because the proposed estimator based on the maximum likelihood criterion uses the noise model with higher accuracy, it still gets the minimum SV and it has an improvement of at least $8.6 \%$ compared with LAV. 
Chapter 4. Robust Power System State Estimation Using $t$-Distribution Noise Model $^{2}$

To further verify the performance of the proposed estimator, a large-scale system is used in the simulation. The PMUs in the IEEE 118-bus system are placed according to [91], where a total number of 108 voltage measurements and 366 current measurements are considered. The pdf of measurement noise is assumed to be the same as given in (4.4) with $\xi_{i}=3, \sigma_{i}^{t}=0.005$. The system data are obtained from [92]. The variance results are given in Table 4.3, where our method still gets the minimum SV, $136 \times 10^{-6}$, and it has an improvement of at least $17.2 \%$ compared with MS estimator using LS fit.

Table 4.3: Variances of the state estimates of the different estimators in the IEEE 118-bus system with PMU measurements.

\begin{tabular}{|c|c|c|c|c|c|c|c|c|c|c|}
\hline \multirow[t]{2}{*}{ Noise } & \multirow[t]{2}{*}{ Estimator } & \multirow[t]{2}{*}{ WLS } & \multicolumn{2}{|c|}{$\mathrm{QL}$} & \multicolumn{2}{|c|}{ SR } & \multicolumn{2}{|c|}{$\mathrm{MS}$} & \multirow[t]{2}{*}{ LAV } & \multirow{2}{*}{$\begin{array}{c}\text { Our } \\
\text { method }\end{array}$} \\
\hline & & & $\begin{array}{l}\text { LS } \\
\text { fit }\end{array}$ & $\begin{array}{c}\text { ML } \\
\text { fit }\end{array}$ & $\begin{array}{c}\text { LS } \\
\text { fit }\end{array}$ & $\begin{array}{c}\text { ML } \\
\text { fit }\end{array}$ & $\begin{array}{l}\text { LS } \\
\text { fit }\end{array}$ & $\begin{array}{c}\text { ML } \\
\text { fit }\end{array}$ & & \\
\hline \multirow[b]{2}{*}{$t_{3}$} & SV & 250 & 170 & 196 & 166 & 189 & 165 & 185 & 171 & 136 \\
\hline & $\begin{array}{l}\text { our method } \\
\text { improve- } \\
\text { ment(\%) }\end{array}$ & 45.7 & 21.5 & 30.1 & 17.8 & 27.4 & 17.2 & 25.8 & 19.6 & - \\
\hline
\end{tabular}

Variances unit: $\times 10^{-6}$.

In addition, the simulation runs using Matlab version R2012b on a $i 7$ Windows 10 laptop with 8 GB RAM to test the computational time. The LAV estimator is built up according to the method provided by [4] and is conducted based on the matlab sub-function provided in GUROBI example. The computational time values of different estimators in the IEEE 118-bus system are given in Table 4.4. The LAV estimator takes the least time, $0.084 \mathrm{~s}$, but its SV is bigger than our method. Moreover, our method is almost 4 times faster than the MS estimator, because the matrix $\left(\bar{H}^{T} \Omega \bar{H}\right)^{-1}$ in our proposed estimator can be precomputed to save some computation time. 
Table 4.4: The computational time (per step) of different estimators in the IEEE 118-bus system.

\begin{tabular}{|c|c|}
\hline Model & $\begin{array}{c}\text { IEEE 1118-bus system } \\
\text { with PMU measurements } \\
(\mathrm{s})\end{array}$ \\
\hline WLS & 0.105 \\
\hline QL(LS fit) & 1.057 \\
\hline SR(LS fit) & 1.792 \\
\hline MS(LS fit) & 1.584 \\
\hline LAV & 0.084 \\
\hline Our method & 0.446 \\
\hline
\end{tabular}

\subsubsection{Example 2: Experiment in NTU Microgrid}

Experiments in a lab microgrid at Nanyang Technological University (NTU) have been done. The lab microgrid has a total of 13 buses which form a circuit loop. There are several generation sources and loads currently installed for the microgrid. For simplicity, one programmable source and one load are used in the microgrid, as shown in Figs. 4.4 and 4.5. The programmable source and the load are connected to Bus 7 and Bus 4 respectively. Measurement data is sampled at intervals of 5 seconds. The system provides a total of 31 measurements, which are composed of 1 voltage magnitude measurement, 2 pairs of power injection and 13 pairs of power flow. The proposed estimator estimates the bus voltage phasor every 30 seconds.

In the experiment, the measurements are collected from the microgrids, after which a gross error is added to the measurement (the real power flow from Bus 3 to Bus 2) at $k=300$ to simulate the outliers. We collect all the measurements and use the $t$ distribution and Gaussian distribution to fit them, respectively. We find that the $t$ distribution matches the histogram better than that of Gaussian distribution. Take the raw measurement (the real power flow from Bus 3 to Bus 2) as an example, it is clear that the curve using t approximation fits the random variables better, as shown in Fig. 4.6. All the three state estimators obtain the estimated results by using the measurement including outliers. However, due to the better approximation of the 
Chapter 4. Robust Power System State Estimation Using t-Distribution Noise Model $^{2}$

noise model, the proposed estimator based on the maximum likelihood criterion can provide better estimated results. The SV value of the WLS is $3.20 \times 10^{-8}$, the SV value obtained from the MS estimator is $2.97 \times 10^{-8}$ and the one obtained from the proposed estimator is the smallest, $2.90 \times 10^{-8}$. The proposed estimator gets the better estimation result can also be verified from Fig. 4.7. Smaller excursions of the outputs are achieved by the proposed robust estimator in response to outliers, while the results of WLS and MS jump sharply.

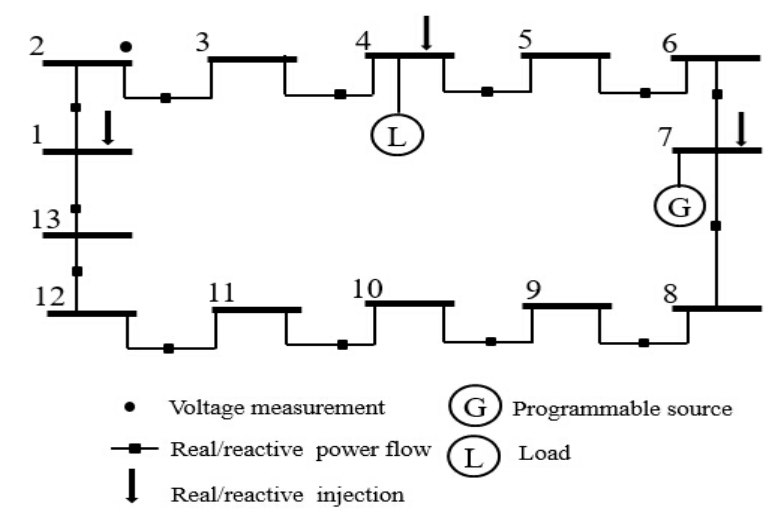

Figure 4.4: Microgrid with 1 programmable source and 1 load.

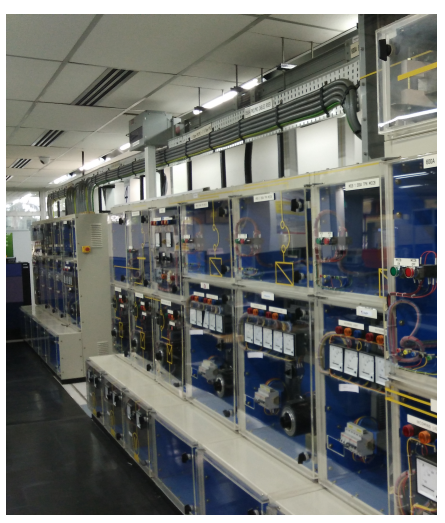

Figure 4.5: Equipments used in the microgrid. 


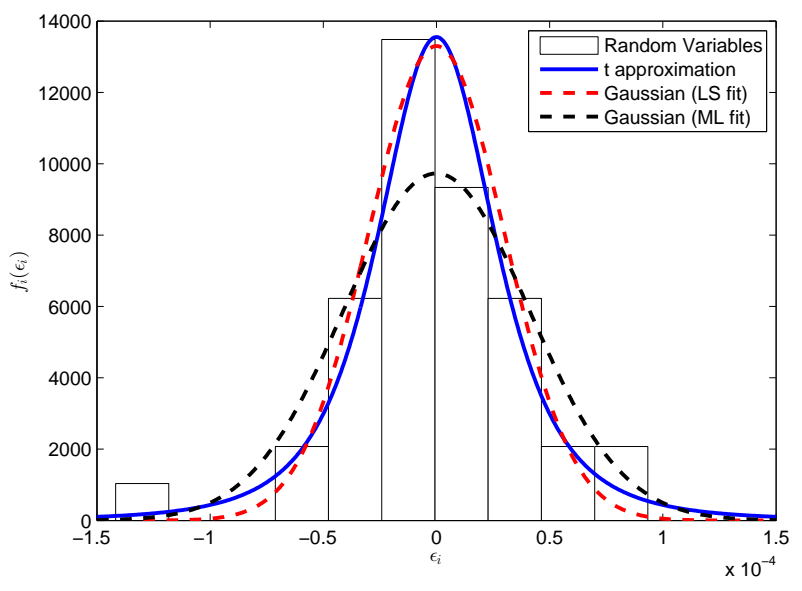

Figure 4.6: The $t$ distribution with $\xi_{i}=3, \sigma_{i}^{t}=4 \times 10^{-5}$ and Gaussian distribution with $\sigma_{i}=3.0 \times 10^{-5}$ and $\sigma_{i}=4.2 \times 10^{-5}$ are used to fit the raw measurement (the real power flow from Bus 3 to Bus 2) collected from microgrid.

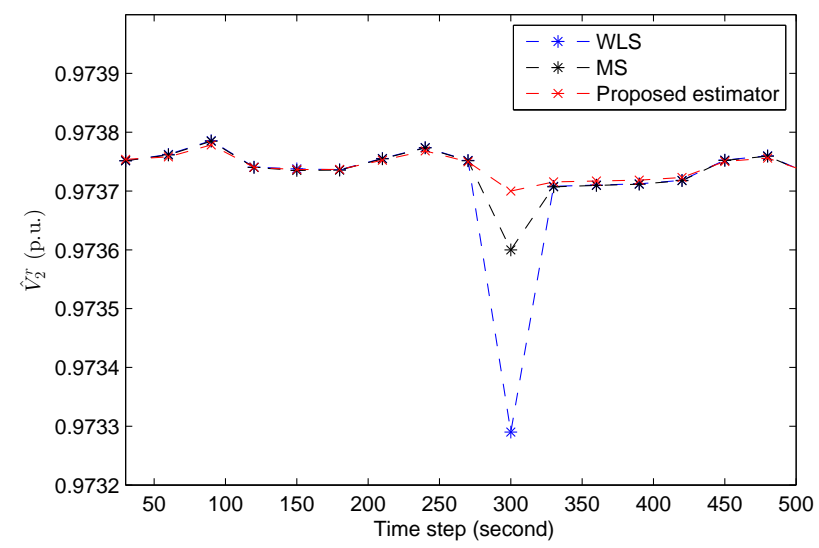

Figure 4.7: The estimated result $\hat{V}_{2}^{r}$ when outliers occur in measurement (the real power flow from Bus 3 to Bus 2). 
Chapter 4. Robust Power System State Estimation Using t-Distribution Noise Model $^{2}$

\subsection{Summary}

In this chapter, a robust estimator based on the maximum likelihood criterion is proposed. The $t$-distribution pdf is used to fit the measurement noise instead of making the usual Gaussian assumption. The influence function is employed, and the maximum likelihood optimization problem can be solved iteratively so that the existing hardware and software for robust estimators can be directly used to implement the proposed estimator. Only some equations are modified and no more devices are added. It is verified that there exists a link between the key equation used by the proposed estimator and the one by WLS. Due to the better approximation of the noise model, the proposed estimator is expected to provide better estimated results, compared with the results obtained from the conventional robust state estimators such as the Least Absolute Value, Quadratic-Linear, Square-Root and Multiple-Segment estimator. The simulations on the IEEE 14-bus and 118-bus systems and experiment on the microgrid show the evidence that the variances of the estimated results from the proposed estimator are smaller than other estimators. 


\section{Chapter 5}

\section{Moving Horizon Estimation for Power Systems ${ }^{3}$}

This chapter will firstly focus on the constrained moving horizon estimation (MHE) for power systems with linear measurement model. The MHE aims to solve at each time instant an optimization problem by using a limited amount of most recent information [56]. The states are estimated by minimizing an overall objective function which consists of measurement model error, process model error, and error in the state estimate at the beginning of the window [27]. The constraints on states can also be included explicitly in the optimization process. This can overcome issues such as the suboptimal estimates or instability of the error dynamics [28,59]. In Chapter 3, the traditional robust estimators can alleviate the influence of outliers by adjusting the weights of measurement errors $[6,24,50,51]$. By combining the constrained MHE and adjusting the weighting matrix in the cost function, this chapter proposes a re-weighted MHE (RMHE) algorithm for robust PSSE. Next, the iterated RMHE (iRMHE) algorithm for power systems with nonlinear measurement model

\footnotetext{
${ }^{3}$ Part of the materials in Chapter 5 is taken from paper "Tengpeng Chen. Robust State Estimation for Power Systems via Moving Horizon Strategy. Sustainable Energy, Grids and Networks, 10: 46-54 (2017)."
} 
is then derived within the framework of constrained RMHE. In order to accelerate the performance of RMHE, the Alternating Direction Method of Multipliers (AD$\mathrm{MM}$ ) is adopted to solve the quadratic programming (QP) problem arising from RMHE. The ADMM is a powerful algorithm for solving structured convex optimization problems. It provides a structured way of decomposing a large problem into smaller-subproblems, and coordinates the solutions of subproblems efficiently to obtain the optimal solution $[63,101]$.

\subsection{Measurement Model and State Equation}

For easy reference, the measurement model presented in Chapter 3 for PSSE is repeated here:

$$
\left\{\begin{array}{cc}
z_{k}=H x_{k}+v_{k} & \text { linear } \\
y_{k}=h\left(x_{k}\right)+v_{k} & \text { nonlinear }
\end{array}\right.
$$

where $k$ is the time index, $z \in \mathbb{R}^{m}$ is the measurement vector and $x \in \mathbb{R}^{n}$ is the state vector. $h(x)$ is a nonlinear measurement function of $x$, and $v \in \mathbb{R}^{m}$ is the measurement noise with covariance $R$. The MHE for the linear and nonlinear measurement model will be presented in different sections.

Traditionally a power system may be considered as a quasi-static system $[85,86]$. Here for generality, we consider the following state equation

$$
x_{k+1}=A x_{k}+w_{k}
$$

where $w$ is the process model noise whose covariance is $Q$. 


\subsection{MHE for Power Systems with Linear Mea- surement Model}

\subsubsection{MHE}

The MHE algorithm in [56] is applied to a PSSE problem. The measurement model (5.1) and the process model (5.2) are assumed to subject to the constraints $x_{k} \in \mathbb{X}$, where $\mathbb{X}$ is a polytope defined by a set of linear inequalities. A favorable property of MHE is its ability to handle the constraints explicitly. This feature is useful from an engineering point of view since additional information about the system is often known in the form of linear inequality constraints. For example, the bus voltage magnitudes in a power system under normal operations are usually around 1 p.u. (per unit). It will be demonstrated in this chapter that incorporating this prior knowledge into the estimation process could improve the performance of the state estimator.

The classical least-squares theory is used to minimize the errors from time step 0 to $t$ and formulate a constrained state estimation problem as follows:

$$
\Theta_{t}^{*}=\min _{x_{0},\left\{w_{k}\right\}_{k=0}^{t-1}} \Psi_{t}\left(x_{0},\left\{w_{k}\right\}\right)
$$

subject to:

$$
\begin{aligned}
& x_{k+1}=A x_{k}+w_{k}, \quad k=0, \ldots, t-1 \\
& z_{k}=H x_{k}+v_{k}, \quad k=0, \ldots, t \\
& x_{k} \in \mathbb{X}
\end{aligned}
$$


where $\bar{x}_{0}$ is the initial estimate of $x_{0}$. The function $\Psi_{t}\left(x_{0},\left\{w_{k}\right\}\right)$ is given by

$$
\Psi_{t}\left(x_{0},\left\{w_{k}\right\}\right)=\frac{1}{2} \sum_{k=0}^{t-1}\left\|w_{k}\right\|_{Q^{-1}}^{2}+\frac{1}{2} \sum_{k=0}^{t}\left\|v_{k}\right\|_{R^{-1}}^{2}+\frac{1}{2}\left\|x_{0}-\bar{x}_{0}\right\|_{P_{0}^{-1}}^{2}
$$

where $\left\{w_{k}\right\}_{k=0}^{t-1}$ denotes the sequence $\left\{w_{0}, \cdots, w_{t-1}\right\}$. The positive definite matrix $P_{0}$ is the initial covariance of state. $\|\cdot\|_{S}^{2}$ represents the square of the weighted Euclidean norm of a vector, e.g., $\|w\|_{S}^{2}=w^{T} S w$, where $S$ is a positive definite matrix.

According to [56,102], the above estimation method of $(5.3)$ is the so-called full information estimation without dropping old measurements. The estimation result without constraints is the same as that of Kalman filter. However, the computation load of (5.3) becomes bigger and bigger as the time step $t$ increases. This drawback makes it unsuitable for a real-time problem. Similar to the method that the model predictive control (MPC) uses a fixed horizon to calculate the next control move [103], MHE removes this difficulty by considering the most recent $N+1$ measurement data $\left\{z_{k}\right\}_{t-N}^{t}$ as shown in Fig. 5.1 to estimate states $\left\{x_{k}\right\}_{t-N}^{t}$. When the new measurements come, MHE shifts the window to discard the oldest set of measurements and to include the new data. Breaking the time interval into two parts $T_{1}=[0, \cdots, t-N-1]$ and $T_{2}=[t-N, \cdots, t]$, of the object function (5.5) is rewritten as follows:

$$
\begin{aligned}
\Psi_{t}\left(x_{0},\left\{w_{k}\right\}\right)= & \frac{1}{2} \sum_{k=0}^{t-N-1}\left\|w_{k}\right\|_{Q^{-1}}^{2}+\frac{1}{2} \sum_{k=0}^{t-N-1}\left\|v_{k}\right\|_{R^{-1}}^{2} \\
& +\frac{1}{2} \sum_{k=t-N}^{t-1}\left\|w_{k}\right\|_{Q^{-1}}^{2}+\frac{1}{2} \sum_{k=t-N}^{t}\left\|v_{k}\right\|_{R^{-1}}^{2}+\frac{1}{2}\left\|x_{0}-\bar{x}_{0}\right\|_{P_{0}^{-1}}^{2}
\end{aligned}
$$

The MHE estimates the sequence $\left\{x_{k}\right\}_{k=t-N}^{t}$ only, the full information estimation 


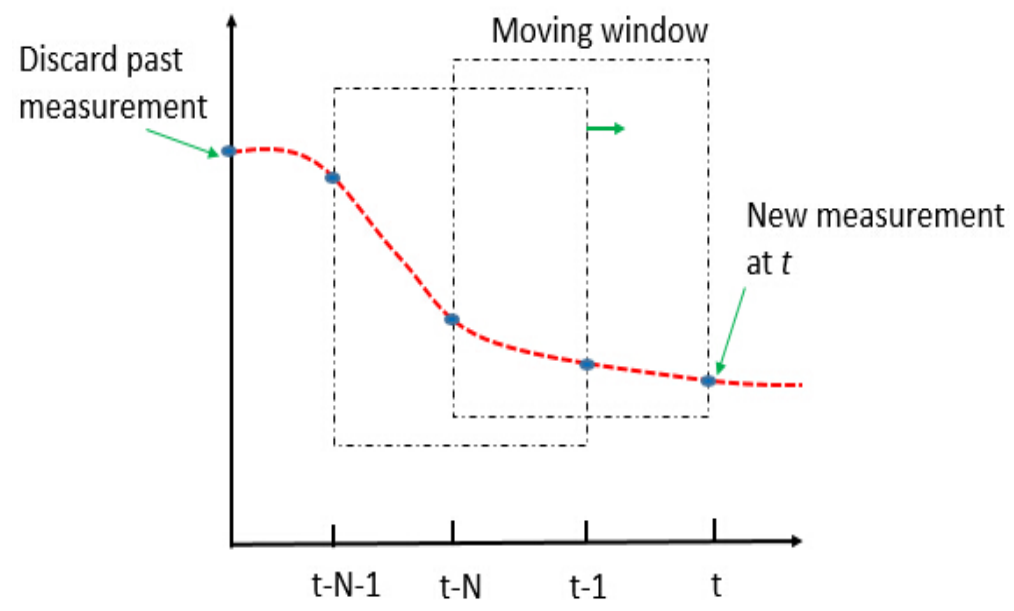

Figure 5.1: Moving horizon window.

(5.3) with the cost function (5.6) can be reformulated as follow:

$$
\Theta_{t}^{*}=\min _{x_{t-N},\left\{w_{k}\right\}_{k=t-N}^{t-1}} \Psi_{t}\left(x_{t-N},\left\{w_{k}\right\}\right)
$$

subject to:

$$
\begin{aligned}
& x_{k+1}=A x_{k}+w_{k}, \quad k=t-N, \ldots, t-1 \\
& z_{k}=H x_{k}+v_{k}, \quad k=t-N, \ldots, t \\
& x_{k} \in \mathbb{X} .
\end{aligned}
$$

The function $\Psi_{t}\left(x_{t-N},\left\{w_{k}\right\}\right)$ is in the form of

$$
\Psi_{t}\left(x_{t-N},\left\{w_{k}\right\}\right)=\frac{1}{2} \sum_{k=t-N}^{t}\left\|v_{k}\right\|_{R^{-1}}^{2}+\frac{1}{2} \sum_{k=t-N}^{t-1}\left\|w_{k}\right\|_{Q^{-1}}^{2}+\Phi_{t-N}
$$

The above function consists of three terms. The first term $\sum_{k=t-N}^{t}\left\|v_{k}\right\|_{R^{-1}}^{2}$ is the error between the measurement model prediction and the raw measurement. The second term $\sum_{k=t-N}^{t-1}\left\|w_{k}\right\|_{Q^{-1}}^{2}$ is the error between the estimated state $x_{k}, k=t-$ $N, \ldots, t-1$ and its process model. In the following, the notation $t-N \mid t-N-1$ 
means the time step for prediction from step $t-N-1$ to $t-N$. The last term $\Phi_{t-N}$ in (5.8) is the arrival cost and it is given by

$$
\Phi_{t-N}=\frac{1}{2}\left\|x_{t-N}-\hat{x}_{t-N \mid t-N-1}\right\|_{P_{t-N \mid t-N-1}^{-1}}^{2}
$$

where $\hat{x}_{t-N \mid t-N-1}$ represents the prior MHE estimation at time step $(t-N)$. The arrival cost (or called "initial penalty") collects the past data information. There does not exist the algebraic equation for the arrival cost when the process model is nonlinear or when constraints are considered [104]. A common remedy to this problem is to find an approximate algebraic expressions, $P_{t-N \mid t-N-1}$ as suggested by [57]:

$$
\begin{aligned}
P_{t-N \mid t-N-1}=\quad & A P_{t-N-1 \mid t-N-2} A^{T}-A P_{t-N-1 \mid t-N-2} H^{T} \times \\
& \times\left(R+H P_{t-N-1 \mid t-N-2} H^{T}\right)^{-1} H P_{t-N-1 \mid t-N-2} A^{T}+Q
\end{aligned}
$$

The optimizers to (5.7) is the pair $\left(\hat{x}_{t-N},\left\{\hat{w}_{k}\right\}_{k=t-N}^{t-1}\right)$, and that allows to compute the sequence $\left\{x_{k}\right\}_{t-N}^{t}$ by using (5.2). The state estimation at time step $t$ is obtained as follows:

$$
\hat{x}_{t}=A^{N} \hat{x}_{t-N}+\sum_{j=t-N}^{t-1} A^{t-j-1} \hat{w}_{j}
$$

The algorithm of MHE is presented in the following pseudo-code:

MHE Algorithm for Power Systems:

1. Initialization: Set $\hat{x}_{1 \mid 0}=\mu_{0}$, where $\mu_{0}$ is given. Choose the length $N$, the covariance matrices $Q, R, P_{1 \mid 0}$.

2. Main procedure: 
Obtain the estimated results $\hat{x}_{t}$ :

while $t \geq 1$ do

(a) Whenever $t \leq N, t-N$ is replaced by 1 in (5.8), and $\Phi_{t-N}=\frac{1}{2} \| x_{1}-$ $\hat{x}_{1 \mid 0} \|_{P_{1 \mid 0}^{-1}}^{2}$.

(b) Whenever $t>N$, use (5.8).

(c) Calculate $\hat{x}_{t}$ by solving (5.7) and using (5.11).

(d) $t \leftarrow t+1$.

end while

\subsubsection{Re-weighted MHE}

Chapter 3 shows that the traditional robust estimators can alleviate the influence of outliers by adjusting the weights of measurement errors. By combining the constrained MHE and adjusting the weighting matrix in the cost function of MHE, a re-weighted MHE (RMHE) is proposed for robust PSSE. The RMHE objective function at iteration index $q$ within time step $t$ is the same as (5.8), but the weighting matrix $R$ is replaced with $R_{k}^{q}$ given as

$$
\begin{aligned}
& R_{k}^{q}=\operatorname{diag}\left(R_{1, k}^{q}, \ldots, R_{m, k}^{q}\right), \\
& R_{j, k}^{q}=W_{j, k}^{-1}= \begin{cases}\sigma_{j}^{2} & \left|e_{j, k}\right| \leq a_{j} \sigma_{j} \\
\frac{\sigma_{j}\left|e_{j, k}\right|}{a_{j}} & a_{j} \sigma_{j}<\left|e_{j, k}\right| \leq b_{j} \sigma_{j} \\
\frac{\left.\left(r_{j}-b_{j}\right) \sigma_{j}^{2}\left|e_{j, k}\right|\right)}{a_{j}\left(r_{j} \sigma_{j}-\left|e_{j, k}\right|\right)} & b_{j} \sigma_{j}<\left|e_{j, k}\right| \leq r_{j} \sigma_{j} \\
+\infty & r_{j} \sigma_{j}<\left|e_{j, k}\right|\end{cases}
\end{aligned}
$$


in which the measurement residuals are given by

$$
e_{i, k}=z_{i, k}-H_{i} \hat{x}_{k}^{q-1}, i=1, \ldots, m ; k=t-N, \ldots, t
$$

where $\hat{x}_{k}^{q-1}$ denotes the solution at iteration index $q-1$ within time step $k$. In this thesis $R_{k}^{q}$ is updated according to the MS robust estimator and it may also be updated by other robust estimators such as SR.

The RMHE algorithm for power system is listed in the following pseudo-code:

RMHE Algorithm for Power Systems

1. Initialization: Choose an initial estimate $\hat{x}_{1 \mid 0}=\mu_{0}$. Set the length $N$ and the covariance matrices $Q, R_{k}^{q}, P_{1 \mid 0}$, the iterative threshold $\delta_{R M H E}$ and iteration maximum number $q_{\max }$.

2. Main procedure:

while $t \geq 1$ do

\section{(a) repeat}

i. When $t \leq N, t-N$ is replaced by 1 in (5.8), and $\Phi_{t-N}=\frac{1}{2} \| x_{1}-$ $\hat{x}_{1 \mid 0} \|_{P_{1 \mid 0}^{-1}}^{2}$.

ii. Whenever $t>N$, use (5.8).

iii. Set $q=0$.

iv. Set $\hat{x}_{t}^{q}=\hat{x}_{t-1}$. (if $\left.t=1, \hat{x}_{1}^{0}=\hat{x}_{1 \mid 0}\right)$

v. $q \leftarrow q+1$.

vi. Calculate $R_{k}^{q}$ using (5.12).

vii. Solve (5.7) and use (5.11) for $\hat{x}_{t}^{q}$.

(b) until $\left(\max \left(\left|\hat{x}_{t}^{q}-\hat{x}_{t}^{q-1}\right|\right) \leq \delta_{R M H E}\right)$ or $q=q_{\max }$. 
(c) $t \leftarrow t+1$.

end while

As presented in Chapter 3, the cost functions for the traditional robust estimators such as MS and SR estimator only include the errors between the measurement model prediction and the raw measurement, while the cost function for RMHE are consisted of three parts (see (5.8)). If the covariance matrix $Q \rightarrow \infty, P_{t-N \mid t-N-1}$ will also tend to $\infty$ according to (5.10). The last two terms in the right side of (5.8) tend to 0 . Moreover, if the length is $N+1=1$, the RMHE without constraints will reduce to a MS estimator. The solution of RMHE is totally the same as MS's, that is,

$$
\hat{x}_{t}=\left(H^{T}\left(R_{t}^{q}\right)^{-1} H\right)^{-1} H^{T}\left(R_{t}^{q}\right)^{-1} z_{t}
$$

where $R_{t}^{q}$ is updated by (5.12). Note that the RMHE can also be reduced to the SR estimator if the matrix $\left(R_{t}^{q}\right)^{-1}$ is updated by (3.14).

Derivations for MHE with nonlinear measurement model are provided in next section.

\subsection{MHE for Power Systems with Nonlinear Mea- surement Model}

One easy way to exploit an optimal solution to nonlinear model is to linearize it with the operating point and apply optimal linear state estimation to these linearized functions [103]. The general extended Kalman filter adopt this basic idea. For PSSE, the estimated result $\hat{x}_{k}$ is obtained by solving the MHE problem iteratively. The resulting algorithm is named as 'iterated MHE' or more compactly 'iMHE' [61]. The 
immediate iterative result $\hat{x}_{k}^{q-1}$ is taken as an operating point to linearize the model. The first-order Taylor series expansion is then given as

$$
\begin{aligned}
y_{k} & \approx h\left(\hat{x}_{k}^{q-1}\right)+\left.\frac{\partial h\left(x_{k}\right)}{\partial x_{k}}\right|_{\hat{x}_{k}^{q-1}}\left(x_{k}^{q}-\hat{x}_{k}^{q-1}\right)+v_{k}^{q} \\
& =h\left(\hat{x}_{k}^{q-1}\right)+H_{k}^{q}\left(x_{k}^{q}-\hat{x}_{k}^{q-1}\right)+v_{k}^{q}
\end{aligned}
$$

where

$$
H_{k}^{q}=\left.\frac{\partial h(x)}{\partial x}\right|_{x=\hat{x}_{k}^{q-1}}
$$

A corresponding iterated and re-weighted MHE (iRMHE) optimization problem is cast as

$$
\Theta_{t}^{*}=\min _{x_{t-N}^{q},\left\{w_{k}^{q}\right\}_{k=t-N}^{t-1}} \Psi_{t}\left(x_{t-N}^{q},\left\{w_{k}^{q}\right\}\right)
$$

subject to

$$
\begin{aligned}
& x_{k+1}^{q}=A x_{k}^{q}+w_{k}^{q}, \quad k=t-N, \ldots, t-1 \\
& y_{k}=h\left(\hat{x}_{k}^{q-1}\right)+H_{k}^{q}\left(x_{k}^{q}-\hat{x}_{k}^{q-1}\right)+v_{k}^{q}, \quad k=t-N, \ldots, t \\
& x_{k}^{q} \in \mathbb{X}
\end{aligned}
$$

The following iRMHE objective function at the iteration $q$ within the time step $t$ is then calculated by

$$
\Psi_{t}\left(x_{t-N}^{q},\left\{w_{k}^{q}\right\}\right)=\frac{1}{2} \sum_{k=t-N}^{t}\left\|y_{k}-h\left(x_{k}^{q}\right)\right\|_{\left(R_{k}^{q}\right)^{-1}}^{2}+\frac{1}{2} \sum_{k=t-N}^{t-1}\left\|w_{k}^{q}\right\|_{Q^{-1}}^{2}+\Phi_{t-N}
$$

where the arrival cost $\Phi_{t-N}$ is given in (5.9).

In the nonlinear case the way to calculate $P_{t-N \mid t-N-1}$ from $P_{t-N-1 \mid t-N-2}$ is given 
as:

$$
\begin{aligned}
P_{t-N \mid t-N-1}= & A P_{t-N-1 \mid t-N-2} A^{T}-A P_{t-N-1 \mid t-N-2} H_{t-N-1}^{T} \times \\
& \times\left(R+H_{t-N-1} P_{t-N-1 \mid t-N-2} H_{t-N-1}^{T}\right)^{-1} \times \\
& \times H_{t-N-1} P_{t-N-1 \mid t-N-2} A^{T}+Q
\end{aligned}
$$

where a time-varying $H_{k}$ is used.

The algorithm of iRMHE is presented in the following pseudo-code:

iRMHE Algorithm for Power Systems:

1. Initialization: Choose an initial estimate $\hat{x}_{1 \mid 0}=\mu_{0}$. Choose $N$, the covariance matrices $Q, R, P_{1 \mid 0}$, the iterative threshold $\delta_{i R M H E}$, and the maximum index $q_{\max }$.

2. Main procedure:

Obtain the estimated results $\hat{x}_{t}$ :

while $t \geq 1$ do

(a) repeat

i. Whenever $t \leq N, t-N$ is replaced by 1 in (5.16), $\Phi_{t-N}=\frac{1}{2} \| x_{1}-$ $\hat{x}_{1 \mid 0} \|_{P_{1 \mid 0}^{-1}}^{2}$.

ii. Whenever $t>N$, use (5.16);

iii. Set $q=0$;

iv. Set $\hat{x}_{t}^{q}=\hat{x}_{t-1}\left(\right.$ if $\left.t=1, \hat{x}_{1}^{0}=\hat{x}_{1 \mid 0}\right)$;

v. $q \leftarrow q+1$.

vi. Calculate $H_{t}^{q}$ using (5.14);

vii. Calculate $R_{k}^{q}$ using (5.12).

viii. Solve (5.15) and use (5.11) for $\hat{x}_{t}^{q}$; 
(b) until $\left(\left\|\hat{x}_{t}^{q}-\hat{x}_{t}^{q-1}\right\| \leq \delta_{i R M H E}\right)$ or $q=q_{\max }$

(c) $t \leftarrow t+1$.

end while

If the covariance matrix $Q \rightarrow \infty, P_{t-N \mid t-N-1}$ will also tend to $\infty$. The last two terms in the right side of (5.16) tend to 0 . Moreover, when the horizon length $N+1$ is 1 and the weighting matrix $R_{k}^{q}$ is chosen as a constant matrix $R$, equation (5.16) reduces to

$$
\Psi_{t-N}\left(x_{t}^{q}\right)=\left\|y_{t}-h\left(x_{t}^{q}\right)\right\|_{R^{-1}}^{2}
$$

which is the same as WLS's minimization equation (A.2). In this case, the iRMHE without constraint is equivalent to WLS.

\subsection{Alternating Direction Method of Multipliers for MHE}

The moving horizon estimation problem can be posed in the framework of quadratic programming (QP). The Alternating Direction Method of Multipliers (ADMM) is a powerful algorithm for solving structured convex optimization problems. It provides a structured way of decomposing very large problems into smaller-subproblems that can be solved efficiently [101]. The ADMM can be viewed as an attempt to blend the benefits of dual decomposition and augmented Lagrangian methods for constrained optimization [63]. This section only focuses on applications rather than theory, and the main goal is to take ADMM as a "toolbox" to solve the MHE optimization problem. 


\subsubsection{Alternating Direction Method of Multipliers}

According to [63], the ADMM algorithm can be used to solve the problems in such form

$$
\min _{\mathbf{x}, \tau} \bar{f}(\mathbf{x})+\overline{\mathbf{g}}(\tau)
$$

subject to

$$
C \mathbf{x}+B \tau=c
$$

where $\bar{f}$ and $\bar{g}$ are convex function, $\mathbf{x} \in \mathbb{R}^{\bar{n}}, \tau \in \mathbb{R}^{\bar{m}}, C \in \mathbb{R}^{\bar{p} \times \bar{n}}, B \in \mathbb{R}^{\bar{p} \times \bar{m}}$ and $c \in \mathbb{R}^{\bar{p}}$.

The augmented Lagrangian for the ADMM iteration is given as

$$
L_{\rho_{0}}(\mathbf{x}, \tau, \phi)=\bar{f}(\mathbf{x})+\bar{g}(\tau)+\frac{\rho_{0}}{2}\|C \mathbf{x}+B \tau-c\|^{2}+\phi^{T}(C \mathbf{x}+B \tau-c)
$$

where $\rho_{0}$ is the penalty parameter and $\phi$ is Lagrange multipliers.

The ADMM iterations for problem (5.18) are

$$
\begin{aligned}
& \mathbf{x}^{(k+1)}=\underset{\mathbf{x}}{\operatorname{argmin}} L_{\rho_{0}}\left(\mathbf{x}, \tau^{(k)}, \phi^{(k)}\right) \\
& \tau^{(k+1)}=\underset{\tau}{\operatorname{argmin}} L_{\rho_{0}}\left(\mathbf{x}^{(k+1)}, \tau, \phi^{(k)}\right) \\
& \phi^{(k+1)}=\phi^{(k)}+\rho_{0}\left(C \mathbf{x}^{(k+1)}+B \tau^{(k+1)}-c\right)
\end{aligned}
$$

where $(k)$ in the subscript is the iteration index. After setting $u=\phi / \rho_{0}$, the ADMM 
iterations can be given in terms of

$$
\begin{aligned}
& \mathbf{x}^{(k+1)}=\underset{\mathbf{x}}{\operatorname{argmin}}\left\{\bar{f}(\mathbf{x})+\frac{\rho_{0}}{2}\left\|C \mathbf{x}+B \tau^{(k)}-c+u^{(k)}\right\|^{2}\right\} \\
& \tau^{(k+1)}=\underset{\tau}{\operatorname{argmin}}\left\{\bar{g}(\tau)+\frac{\rho_{0}}{2}\left\|C \mathbf{x}^{(k+1)}+B \tau-c+u^{(k)}\right\|^{2}\right\} \\
& u^{(k+1)}=u^{(k)}+C \mathbf{x}^{(k+1)}+B \tau^{(k+1)}-c
\end{aligned}
$$

And the convergence of ADMM is often characterized in terms of the residuals

$$
\begin{aligned}
& \bar{r}^{(k+1)}=C \mathbf{x}^{(k+1)}+B \tau^{(k+1)}-c \\
& \bar{s}^{(k+1)}=\rho_{0} C^{T} B\left(\tau^{(k+1)}-\tau^{(k)}\right)
\end{aligned}
$$

termed the primal and dual residuals, respectively [63].

\subsubsection{Quadratic Programming Problem Formulation of MHE}

In this subsection, the quadratic programming (QP) arising from the MHE is presented. According to (5.8) and (5.18), define

$$
\begin{aligned}
\mathbf{x}= & {\left[x_{t-N}^{T} \cdots x_{t}^{T}\right]^{T} \in \mathbb{R}^{(N+1) n} } \\
Z= & {\left[z_{t-N}^{T} \cdots z_{t}^{T}\right]^{T} \in \mathbb{R}^{(N+1) m} } \\
\bar{Q}= & \Upsilon_{1}^{T}\left(I_{N} \otimes Q^{-1}\right) \Upsilon_{1}^{T}+\tilde{H}^{T}\left(I_{N+1} \otimes R^{-1}\right) \tilde{H}+\Upsilon_{2}^{T} P_{t-N \mid t-N-1} \Upsilon_{2} \\
& \in \mathbb{R}^{(N+1) n \times(N+1) n} \\
\bar{q}^{T}= & -Z^{T}\left(I_{N+1} \otimes R^{-1}\right) \tilde{H}-\Upsilon_{2}^{T} P_{t-N \mid t-N-1} \hat{x}_{t-N \mid t-N-1} \in \mathbb{R}^{1 \times(N+1) n}
\end{aligned}
$$




$$
\begin{aligned}
\tilde{H}= & I_{N+1} \otimes H \in \mathbb{R}^{(N+1) m \times(N+1) n} \\
\mathbb{K}= & \mathbf{1}_{N+1} \otimes \mathbb{X} \\
& {\left[\begin{array}{cccccc}
-A & I_{n} & \mathbf{0} & \cdots & \mathbf{0} & \mathbf{0} \\
\mathbf{0} & -A & I_{n} & \cdots & \mathbf{0} & \mathbf{0} \\
\vdots & \vdots & \vdots & \ddots & \vdots & \vdots \\
\mathbf{0} & \mathbf{0} & \mathbf{0} & \cdots & I_{n} & \mathbf{0} \\
\mathbf{0} & \mathbf{0} & \mathbf{0} & \cdots & -A & I_{n}
\end{array}\right] \in \mathbb{R}^{(N+1) n \times(N+1) n} } \\
\Upsilon_{1}= & {\left[\begin{array}{cccc}
I_{n} & \mathbf{0} & \cdots & \mathbf{0}
\end{array}\right] \in \mathbb{R}^{n \times(N+1) n} } \\
\Upsilon_{2}= &
\end{aligned}
$$

where $\otimes$ is the Kronecker product. Then the cost function of MHE can be reformulated as standard form (5.24), which will be presented in the next subsection.

\subsubsection{Standard ADMM Iterations}

A QP problem arising from the constrained MHE is of the form

$$
\min _{\mathbf{x}} \frac{1}{2} \mathbf{x}^{T} \bar{Q} \mathbf{x}+\bar{q}^{T} \mathbf{x}
$$

subject to

$$
\mathbf{x} \in \mathbb{K} \text { (or to be defined as } C \mathbf{x} \leq c)
$$

The QP problem (5.24) can be put on (5.18) by introducing the slack variables $\tau$ and putting an infinite penalty on negative components of $\tau$ [65],

$$
\min _{\mathbf{x}} \frac{1}{2} \mathbf{x}^{T} \bar{Q} \mathbf{x}+\bar{q}^{T} \mathbf{x}+I_{+}(\tau)
$$


subject to

$$
C \mathbf{x}+\tau=c
$$

where $I_{+}(\cdot)$ is the indicator function of $\tau$

$$
I_{+}(\tau)=\left\{\begin{array}{lc}
0 & \tau \geq 0 \\
+\infty & \text { otherwise }
\end{array}\right.
$$

Now the augmented Lagrangian is given as

$$
L_{\rho_{0}}(\mathbf{x}, \tau, u)=\frac{1}{2} \mathbf{x}^{T} \bar{Q} \mathbf{x}+\bar{q}^{T} \mathbf{x}+I_{+}(\tau)+\frac{\rho_{0}}{2}\|C \mathbf{x}+\tau-c+u\|^{2}
$$

where $u=\phi / \rho_{0}$. The scaled ADMM iterations are given as

$$
\begin{aligned}
\mathbf{x}^{(k+1)} & =-\left(\bar{Q}+\rho_{0} C^{T} C\right)^{-1}\left[\bar{q}+\rho_{0} C^{T}\left(\tau^{(k)}+u^{(k)}-c\right)\right] \\
\tau^{(k+1)} & =\max \left\{0,-C \mathbf{x}^{(k+1)}-u^{(k)}+c\right\} \\
u^{(k+1)} & =u^{(k)}+C \mathbf{x}^{(k+1)}-c+\tau^{(k+1)}
\end{aligned}
$$

\subsubsection{Over-relaxed ADMM Iterations}

In order to improve the convergence properties of the ADMM algorithm, one approach is to use the relaxation technique. The relaxation of (5.28) works by replacing $C \mathbf{x}^{(k+1)}$ in the $\tau$ - and $u$ - updates with $\alpha C \mathbf{x}^{(k+1)}-(1-\alpha)\left(\tau^{(k)}-c\right)$, where $\alpha \in(0,2)$ is a relaxation parameter. The relaxed iterations are given as

$$
\begin{aligned}
\mathbf{x}^{(k+1)} & =-\left(\bar{Q}+\rho_{0} C^{T} C\right)^{-1}\left[\bar{q}+\rho_{0} C^{T}\left(\tau^{(k)}+u^{(k)}-c\right)\right] \\
\tau^{(k+1)} & =\max \left\{0,-\alpha\left(C \mathbf{x}^{(k+1)}-c\right)+(1-\alpha) \tau^{(k)}-u^{(k)}\right\} \\
u^{(k+1)} & =u^{(k)}+\alpha\left(C \mathbf{x}^{(k+1)}-c+\tau^{(k+1)}\right)+(1-\alpha)\left(\tau^{(k+1)}-\tau^{(k)}\right)
\end{aligned}
$$


When $\alpha>1$, this technique is called over-relaxation, while it is called underrelaxation when $\alpha<1$. Experiments in $[105,106]$ suggest that over-relaxation with $\alpha \in[1.5,1.8]$ can improve convergence. In this thesis the ADMM is used as a tool to accelerate the performance of MHE. About the convergence of (5.29), readers can see [65] for details.

\subsection{Simulation Results}

In order to analyze the performance of the proposed RMHE and iRMHE, simulations are conducted on linear and nonlinear measurement models respectively. In a traditional power system, when the PMUs are used, the measurements are collected at a high rate and the state variables does not change significantly due to the small sampling time; When the PMUs are not used, the traditional monitoring technologies implemented in the SCADA system can only take nonsynchronized measurements once every few seconds. However, the estimates are usually updated once every few minutes in order to reduce the computational complexity required in implementing state estimation [8]. The matrix $A$ is simplified as an identity matrix according to $[86,91]$, while it is chosen as $A=\operatorname{diag}_{n}\{0.98\}$ in [107]. For simplicity, in this thesis we choose $A$ as an identity matrix to implement our algorithms. For easy reference, the probability density functions of the "normal" noise and outliers, and the parameters of the robust state estimators are summarized in Tables 5.1 and 5.3.

\subsubsection{Simulations Based on Linear Measurement Model}

In this subsection, the IEEE 14-bus (Fig. 3.4) and 30-bus (Fig. 3.8) systems installed with PMUs are used firstly to test RMHE. For the voltage measurements $z_{i, k}$, the noise $v_{i}$ is associated with the pdf given in (3.34) with $\sigma_{i}=0.005$. For the 
current measurements $z_{i, k}$, the noise $v_{i}$ is associated with the pdf given in (3.35) with $\sigma_{i}=0.01$.

Define the Mean Square Error (MSE) [4] for the estimation results at time step $k$ as

$$
\mathrm{MSE}_{k}=\sqrt{\frac{1}{n}\left\|\hat{x}_{k}-x_{k}\right\|^{2}}
$$

Denote the Average of Mean Square Error (AMSE) for $k \in[1,60]$ to evaluate the estimation accuracy:

$$
\operatorname{AMSE}=\frac{1}{60} \sum_{k=1}^{60} \sqrt{\frac{1}{n}\left\|\hat{x}_{k}-x_{k}\right\|^{2}}
$$

$\mathbf{1}_{n}$ and $\mathbf{0}_{n}$ represent the $n$-dimensional column vector with all ones or zeros, respectively. The initialization parameters of the RMHE algorithm for the IEEE 14-bus system are listed as follows:

- The initial state vectors $\mu_{0}=\left[\mathbf{1}_{14}^{T}, \mathbf{0}_{14}^{T}\right]^{T}$.

- The initial covariance matrix: $P_{1 \mid 0}=10^{2} I_{28}$.

- The noise covariances: $Q=10^{-6} I_{28} ; R=\operatorname{diag}\left(\sigma_{1}^{2}, \ldots, \sigma_{58}^{2}\right)$.

- State constraints: $0.95 \leq V_{j}^{r} \leq 1.1,-0.35 \leq V_{j}^{i m} \leq 0.01$, where $j=1, \ldots, 14$.

For the IEEE 30-bus system, the initialization parameters of RMHE are listed as follows:

- The initial state vectors $\mu_{0}=\left[\mathbf{1}_{30}^{T}, \mathbf{0}_{30}^{T}\right]^{T}$.

- The initial covariance matrix: $P_{1 \mid 0}=10^{2} I_{60}$.

- The noise covariances: $Q=10^{-6} I_{60} ; R=\operatorname{diag}\left(\sigma_{1}^{2}, \ldots, \sigma_{86}^{2}\right)$.

- State constraints: $0.9 \leq V_{j}^{r} \leq 1.1,-0.4 \leq V_{j}^{i m} \leq 0.01$, where $j=1, \ldots, 30$. 
Table 5.1: The AMSE ${ }^{\natural}$ values for different estimators with different parameters in the simulation examples.

\begin{tabular}{|c|c|c|c|c|}
\hline Model & \multicolumn{2}{|c|}{$\begin{array}{c}\text { IEEE 14-bus System, } \\
\text { with PMU Measurements }\end{array}$} & \multicolumn{2}{|c|}{$\begin{array}{c}\text { IEEE 30-bus System } \\
\text { with PMU Measurements }\end{array}$} \\
\hline $\begin{array}{c}\text { Measure- } \\
\text { ment } i\end{array}$ & $1, \ldots, 12$ & $13, \ldots, 58$ & $1, \ldots, 28$ & $29, \ldots, 86$ \\
\hline $\begin{array}{l}\text { Normal } \\
\text { Noise }\end{array}$ & $\begin{array}{c}0.97 \\
* N(0.005)\end{array}$ & $\begin{array}{c}0.97 \\
N(0.01)\end{array}$ & $\begin{array}{c}0.97 \\
N(0.005)\end{array}$ & $\begin{array}{c}0.97 \\
N(0.01)\end{array}$ \\
\hline Outliers & $\begin{array}{c}0.03 \\
N(0.05)\end{array}$ & $\begin{array}{c}0.03 \\
\dagger U(0.1)\end{array}$ & $\begin{array}{c}0.03 \\
N(0.05)\end{array}$ & $\begin{array}{c}0.03 \\
U(0.1)\end{array}$ \\
\hline Estimator & $\ddagger(2.5,3.5,4.5)$ & $(3,4,5)$ & $(2.5,3.5,4.5$ & $(3,4,5)$ \\
\hline$\sharp \operatorname{WLS}(1)$ & \multicolumn{2}{|l|}{3.7} & \multicolumn{2}{|c|}{5.6} \\
\hline $\mathrm{QC}(1)$ & \multicolumn{2}{|c|}{3.0} & \multicolumn{2}{|c|}{4.7} \\
\hline $\mathrm{QL}(1)$ & \multicolumn{2}{|c|}{3.0} & \multicolumn{2}{|c|}{4.7} \\
\hline $\operatorname{SHGM}(1)$ & \multicolumn{2}{|c|}{3.0} & \multicolumn{2}{|c|}{4.7} \\
\hline $\operatorname{MS}(1)$ & \multicolumn{2}{|c|}{2.8} & \multicolumn{2}{|c|}{4.6} \\
\hline $\mathrm{SR}(1)$ & \multicolumn{2}{|c|}{2.8} & \multicolumn{2}{|c|}{4.6} \\
\hline $\operatorname{LAV}(1)$ & \multicolumn{2}{|c|}{3.0} & \multicolumn{2}{|c|}{4.7} \\
\hline $\mathcal{S}_{\mathrm{MHE}}(\infty, 1)^{\text {without }}$ & \multicolumn{2}{|c|}{3.7} & \multicolumn{2}{|c|}{5.6} \\
\hline $\operatorname{RMHE}(\infty, 1)^{\text {without }}$ & \multicolumn{2}{|c|}{2.8} & \multicolumn{2}{|c|}{4.6} \\
\hline $\operatorname{MHE}\left(10^{-5} I, 1\right)^{\text {with }}$ & \multicolumn{2}{|l|}{2.2} & \multicolumn{2}{|c|}{2.9} \\
\hline $\operatorname{RMHE}\left(10^{-5} I, 1\right)^{\text {with }}$ & \multicolumn{2}{|l|}{1.6} & \multicolumn{2}{|c|}{2.4} \\
\hline WLS with $\operatorname{LNR}(3)$ & \multicolumn{2}{|c|}{1.8} & \multicolumn{2}{|c|}{2.6} \\
\hline $\operatorname{IRWLS}(3)$ & \multicolumn{2}{|c|}{1.8} & \multicolumn{2}{|c|}{2.6} \\
\hline Method in Ch. 4 & \multicolumn{2}{|c|}{1.6} & \multicolumn{2}{|c|}{2.5} \\
\hline $\mathrm{QC}(3)$ & \multicolumn{2}{|c|}{1.8} & & \\
\hline $\mathrm{QL}(3)$ & 1.8 & & & \\
\hline $\operatorname{SHGM}(3)$ & 1.8 & & & \\
\hline $\operatorname{MS}(3)$ & 1.7 & & & \\
\hline $\operatorname{SR}(3)$ & 1.7 & & & \\
\hline $\operatorname{LAV}(3)$ & 1.8 & & & \\
\hline $\operatorname{WLAV}(3)$ & 1.8 & & & \\
\hline $\operatorname{MHE}(\infty, 3)^{\text {without }}$ & 3.7 & & & \\
\hline $\operatorname{RMHE}(\infty, 3)^{\text {without }}$ & 2.8 & & & \\
\hline $\operatorname{MHE}\left(10^{-6} I, 3\right)^{\text {with }}$ & 1.7 & & & \\
\hline RMHE $\left(10^{-6} I, 3\right)^{\text {with }}$ & 1.4 & & & \\
\hline Fig. & $5.2,5.3,5$ & $4,5.6$ & & \\
\hline
\end{tabular}

${ }^{\natural}$ AMSE unit: $\times 10^{-3}$.

${ }^{*} N\left(\sigma_{i}\right)=\frac{1}{\sqrt{2 \pi \sigma_{i}^{2}}} e^{-\frac{v_{i}^{2}}{2 \sigma_{i}^{2}}} ;{ }^{\dagger} U\left(\sigma_{i}\right)=\frac{1}{2 \sigma_{i}}$ for $-\sigma_{i} \leq v_{i} \leq \sigma_{i}$.

¥ Parameter $a_{i}$ for QC, QL, SR and SHGM are the first term of the bracket $\left(a_{i}, b_{i}, r_{i}\right)$, while parameters $a_{i}, b_{i}, r_{i}$ for MS are given in the bracket of $\left(a_{i}, b_{i}, r_{i}\right)$.

$\sharp$ Parameter $N+1$ for the traditional estimators are given in the bracket of $(N+1)$.

$\mathcal{S}$ Parameters $Q$ and $N+1$ for MHE and RMHE are given in the bracket of $(Q, N+1)$. The subscript without and with mean "without constraints" and "with constraints" respectively. 
It is quite obvious that when the parameters $a_{i}, b_{i}, r_{i}$ and $Q$ are set to $\infty$, and the horizon length is set as 1, the QC, QL, MS, SR, SHGM, MHE without constraints, and RMHE without constraints reduce to WLS. For example, the MSE results of WLS, MS and RMHE without constraints are shown in Fig. 5.2, where the estimators's results are totally the same.

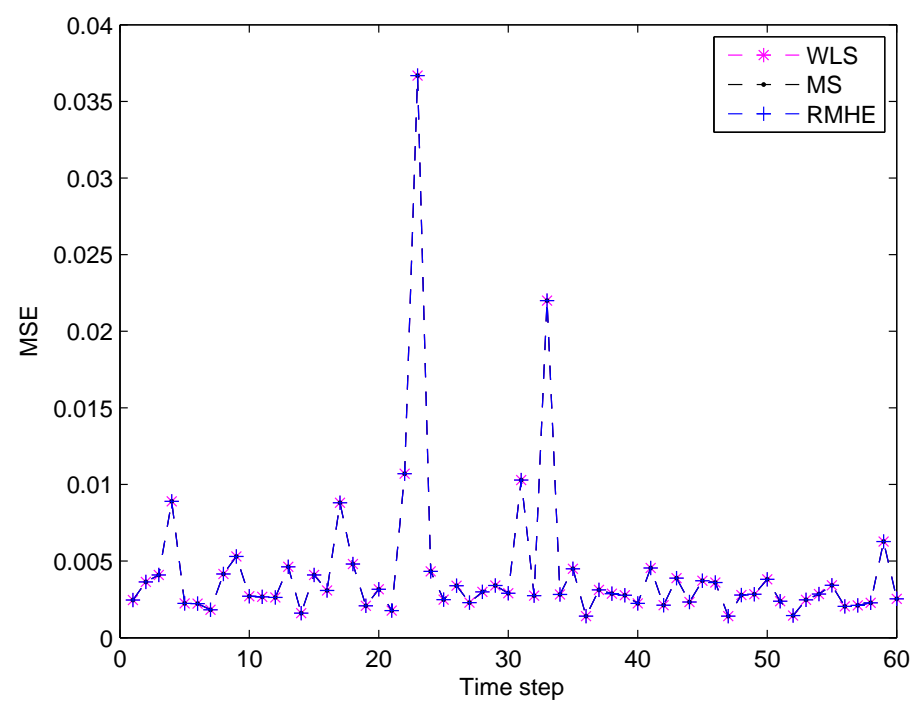

Figure 5.2: Mean square errors of $\operatorname{WLS}(1), \operatorname{MS}(1)$ and $\operatorname{RMHE}\left(Q \rightarrow \infty, R_{k}^{q}=R\right)$ without constraints in the IEEE 14-bus system.

In this simulation, one snapshot of measurements, $k=t$, or 3 sets of measurements i.e. $k=t-2, t-1, t$ are used to calculate the estimated result of $x_{t}$. The AMSE values of different estimators are given in Table 5.1. Take the results of the IEEE 14bus system for explanation, the AMSE value of WLS is $3.7 \times 10^{-3}$. $\operatorname{Both} \operatorname{MHE}(\infty, 1)$ and $\operatorname{MHE}(\infty, 3)$ without constraints reduce to WLS. Even though the horizon length is set as 3 , the sequences $\hat{x}_{t-N}, \ldots, \hat{x}_{t}$ are solved independently and $\hat{x}_{t}$ is actually calculated by using only one set of measurement $z_{t}$, under the setting of $Q \rightarrow \infty$. If the "re-weighted" process is added, the $\operatorname{AMSE}$ value of $\operatorname{RMHE}(\infty, 3)$ is $2.8 \times 10^{-3}$. It is less than that of $\operatorname{MHE}(\infty, 3)$ and that means the "re-weighted" process to reduce influence of outliers works. When the covariance matrix $Q$ is set as $10^{-6} I$ in order 
to make use of 3 sets of measurements, and constraints are considered, the AMSE value of $\operatorname{MHE}\left(10^{-6} I, 3\right)$ is $1.7 \times 10^{-3}$. In order to reduce the influence of outliers further, the "re-weighted" is added to MHE, that is called $\operatorname{RMHE}\left(10^{-6} I, 3\right)$ and its AMSE value is $1.4 \times 10^{-3}$.

According to Table 5.1, the RMHE with constraints is efficient to reduce the influence of outliers and gets the minimum AMSE in the IEEE 14-bus system. Even though the QC, QL, MS, SHGM, SR, iteratively re-weighted least squares(IRWLS), the weighted LAV(WLAV) (see [108]) and WLS with Largest Normalized Residuals (LNR) (see Appendix C for brief introduction) play well, and their AMSE values are all around $1.7 \times 10^{-3}$. However, all of them do not consider the information during the optimization process, i.e., the constraints on states. For the IEEE 14-bus system, when the horizon length is set as $N+1=1$, the AMSE value of the MS estimator is $2.8 \times 10^{-3}$ and the result of the RMHE with constraints is $1.6 \times 10^{-3}$ through Table 5.1. The RMHE has an improvement of $(2.8-1.6) / 2.8 \times 100 \%=43 \%$ compared with the MS estimator. The RMHE has an improvement of $(3.0-1.6) / 3.0 \times 100 \%=47 \%$ compared with LAV. Moreover, When the length is 3, the AMSE value of the MS estimator is $1.7 \times 10^{-3}$, the AMSE value of the LAV estimator is $1.8 \times 10^{-3}$, while the result of RMHE is $1.4 \times 10^{-3}$. The RMHE has improvements of $18 \%$ and $22 \%$ to MS estimator and LAV estimator, respectively. The MSE results are given in Figs. 5.3 and 5.4. It is quite clear that the MSE curve of RMHE is a little lower than that of other estimators.

According to Table 5.1, similar conclusion can be found from the results based on the IEEE 30-bus system. The RMHE in the IEEE 30-bus system is still quite efficient to reduce the influence of outliers. For example, the AMSE value of RMHE $\left(10^{-6} I, 3\right)$ with constraints is $1.7 \times 10^{-3}$ when 3 sets of measurements are used, and it has an improvement of $32 \%$ compared with MS estimator. The MSE results are given in Fig. 5.5. The MSE curve of RMHE with constraints is lower than that of others. 


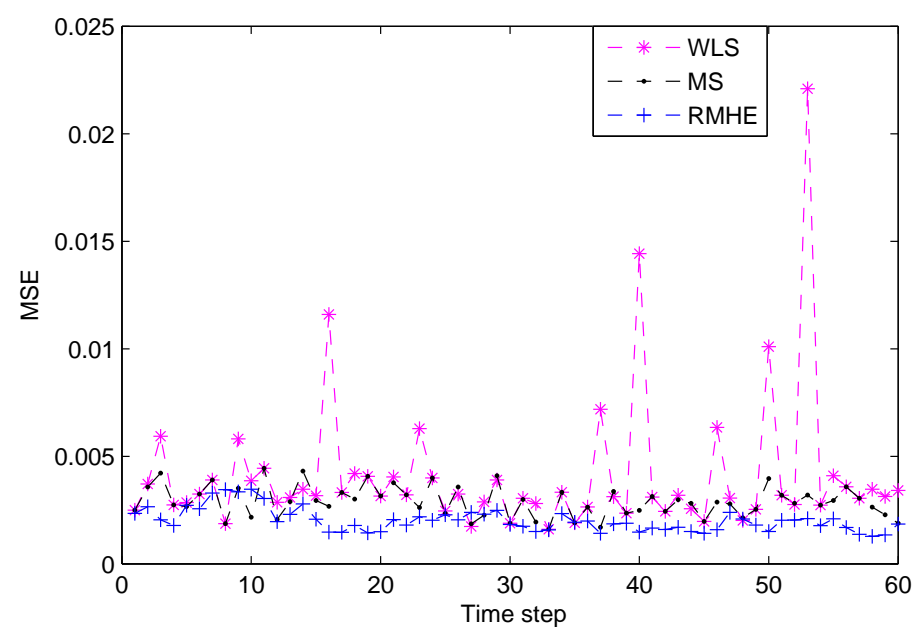

Figure 5.3: Mean square errors of WLS, MS and $\operatorname{RMHE}\left(Q=10^{-5} I, N+1=1\right)$ with constraints in the IEEE 14-bus system.

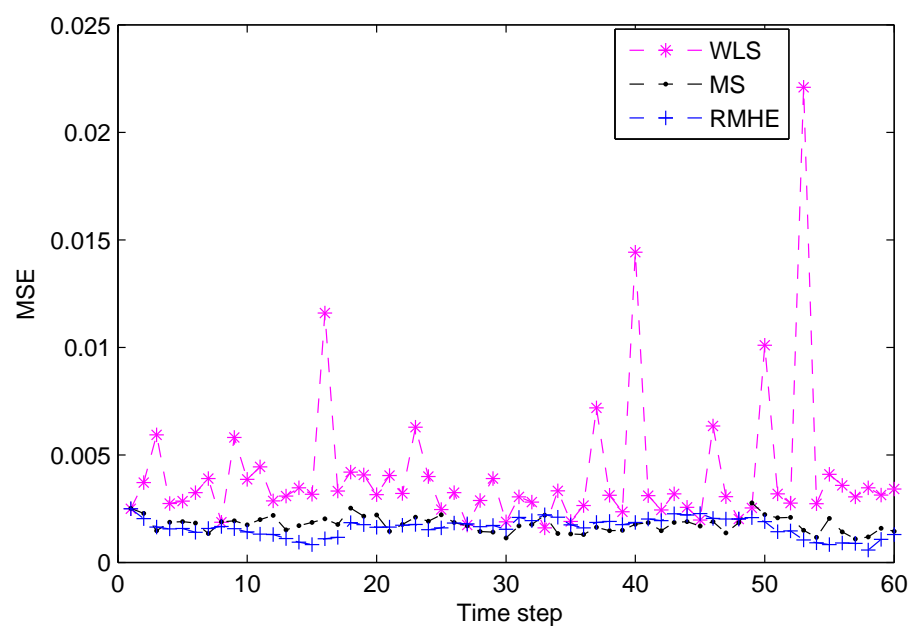

Figure 5.4: Mean square errors of WLS, MS and $\operatorname{RMHE}\left(Q=10^{-6} I, N+1=3\right)$ with constraints in the IEEE 14-bus system. 


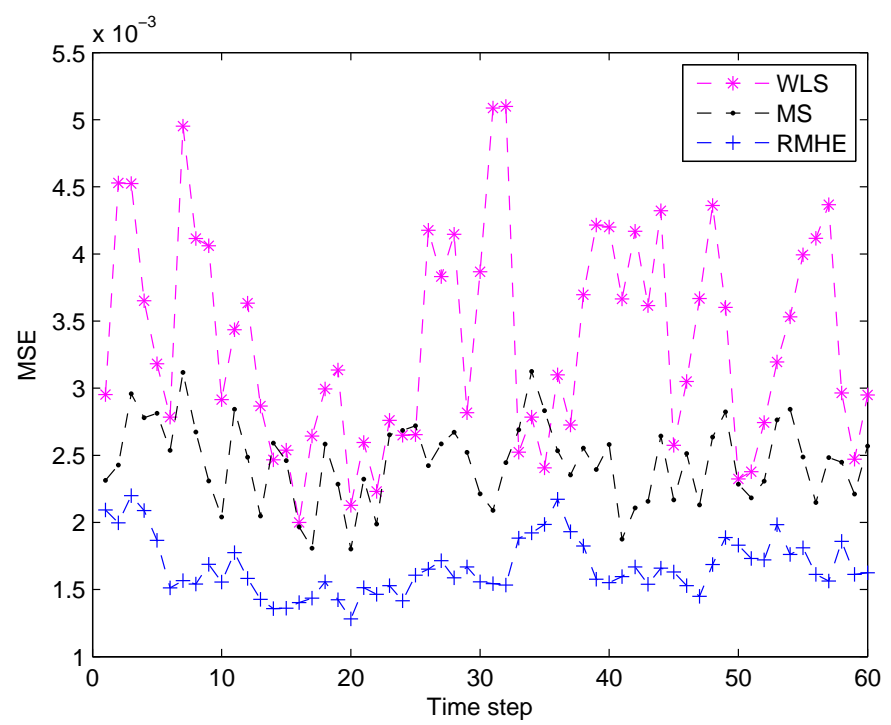

Figure 5.5: Mean square errors of WLS, MS and $\operatorname{RMHE}\left(Q=10^{-6} I, N+1=3\right)$ with constraints in the IEEE 30-bus system.

Multiple bad data items are presented and those occur at time step 23 in the IEEE 14-bus system. Measurements, the real part of Bus 2 voltage phasor and the imaginary part of Bus 7 voltage phasor, are manually changed by $60 \%$. From Fig. 5.6, we can see that WLS (without LNR) is affected seriously by the bad data. The WLS with LNR can detect the relevant bad data and then update the measurements by using C.2. The MS estimator can mitigate the influence of the outliers by reweighting the weights of the measurements errors. The RMHE with constraints can also deal with the bad data by adjusting the weights of the measurement errors and using the constraints. In summary, the robust estimators such as WLS with LNR, MS and RMHE can still get good estimated results.

In addition, the simulation runs using Matlab version R2012b on a $i 7$ Windows 10 laptop with 8 GB RAM to test the computational time. The average computational time (per step) of different estimators are given in Table 5.2. The LAV takes the least time, where $0.012 \mathrm{~s}$ are needed for the LAV estimator to get one set of estimated results. However, the AMSE result of LAV is worse than that of RMHE with constraints, according to Table 5.1. The WLS with LNR takes 0.014 s per step. 


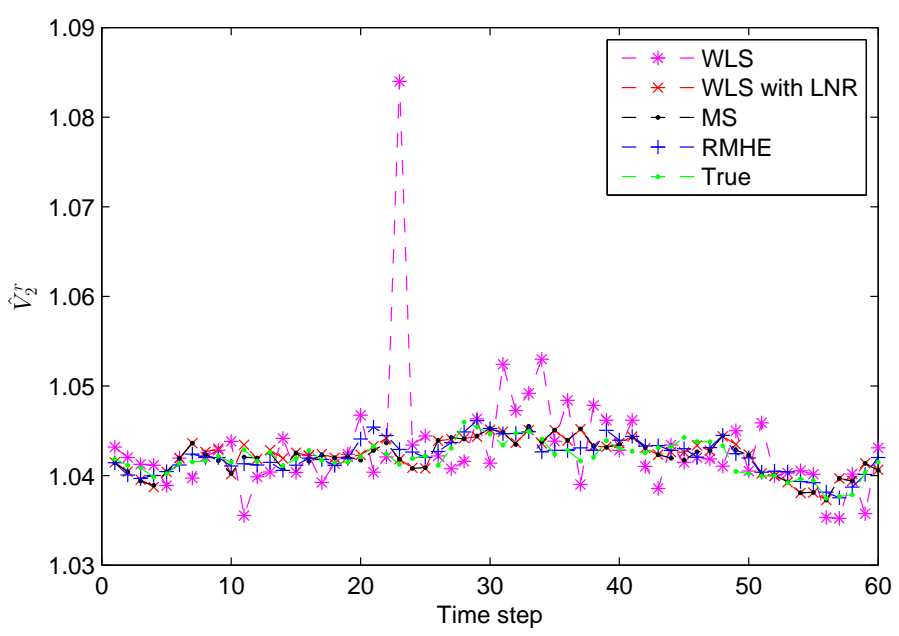

Figure 5.6: Real component of Bus 2 voltage phasor estimated by different estimators in IEEE 14-bus system.

For the traditional robust estimators, the QC spends less time compared with that of QL, MS, SR and SHGM estimator, due to the different chosen function $\rho$ for estimators. The robust estimator proposed in Chapter 4 takes $0.061 \mathrm{~s}$ per step. The system is not large so the time spending in the inverse calculation for the traditional estimators is also small. Even though the proposed estimator in Chapter 4 does not need to recompute the inverse of $\left(\bar{H}^{T} \Omega \bar{H}\right)$, the matrix inverse time is also not less than that of other traditional estimators. When the dimension of $H$ increases in the IEEE 30-bus system, it only takes 0.142 s. The computation time increases slower than that of other estimators such as MS and SR estimator.

The ADMM is used to solve the QP problems arising from MHE and RMHE, and the termination of iteration is set as [63]

$$
\begin{aligned}
\epsilon^{p r i} & =\sqrt{p} \epsilon^{a b s}+\epsilon^{r e l} \max \left\{\left\|C \mathbf{x}^{(k)}\right\|^{2},\left\|\tau^{(k)}\right\|^{2},\|c\|^{2}\right\}, \\
\epsilon^{\text {dual }} & =\sqrt{p} \epsilon^{a b s}+\epsilon^{r e l}\left\|C^{T} u^{k}\right\|^{2}
\end{aligned}
$$

where $\epsilon^{a b s}=10^{-4}$ and $\epsilon^{r e l}=10^{-2}$. The parameters $\rho_{0}$ and $\alpha$ are chosen as 1 and 1.5 respectively [63]. The iteration processes is shown in Fig. 5.7. The iteration will be 
terminated when both $\|\bar{r}\|^{2}$ and $\|\bar{s}\|^{2}$ are less than $\epsilon^{p r i}$ and $\epsilon^{d u a l}$ respectively.
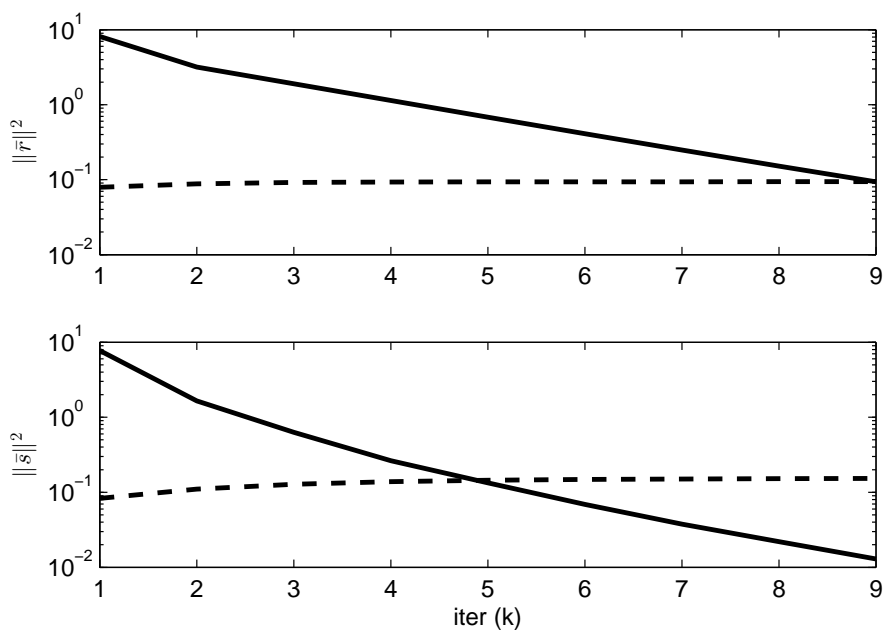

Figure 5.7: $\|\bar{r}\|^{2},\|\bar{s}\|^{2}$ (the solid curve) and $\epsilon^{p r i}, \epsilon^{\text {dual }}$ (the dotted curve).

According to Table 5.2, it is quite clear that the MHE and RMHE using ADMM take much less time than these using the Matlab function "quadprog". That verifies ADMM is a good tool to solve such kind of QP problem. For the IEEE 14-bus system, RMHE using ADMM takes only 0.05 s while the RMHE using "quadprog" spends $0.209 \mathrm{~s}$. In this case RMHE using ADMM is 4 times faster than the one using "quadprog". The MHE using ADMM takes only 0.024 s because it does not need to recalculate the weighting matrix and solve the optimization problem only one time. The RMHE using ADMM takes 0.05 s because it may solve the optimization problems several times so as to obtain the estimated results. Even though the RMHE using ADMM takes a little more time than that of the WLS with LNR and LAV, the accuracy of RMHE is the highest. For the IEEE 30-bus system model, the MS robust estimator takes more time at every step and the average time is $0.304 \mathrm{~s}$. The Method in Chapter 4 does not need to calculate "inverse" at every iteration, it only takes $0.142 \mathrm{~s}$ even though the dimension of $H$ matrix increases. Thanks to ADMM, the RMHE only takes 0.169 s to obtain one set of estimated results. Due to the development of more powerful computation devices, it can enhance more faster 
computation speed of RMHE. As a compromise between the computation speed and the accuracy of estimated results, the RMHE estimator to be implemented in power systems is acceptable.

Table 5.2: The average computational time (per step) of different estimators based on linear measurement model.

\begin{tabular}{|c|c|c|}
\hline Model & $\begin{array}{c}\text { IEEE 14-bus system } \\
\text { with PMU measurements } \\
(\mathrm{s})\end{array}$ & $\begin{array}{c}\text { IEEE 30-bus system } \\
\text { with PMU measurements } \\
(\mathrm{s})\end{array}$ \\
\hline WLS with LNR (3) & 0.014 & 0.057 \\
\hline Method in Ch. 4 & 0.061 & 0.142 \\
\hline QC(3) & 0.019 & 0.061 \\
\hline QL(3) & 0.081 & 0.268 \\
\hline MS(3) & 0.095 & 0.304 \\
\hline SR(3) & 0.115 & 0.340 \\
\hline SHGM(3) & 0.125 & 0.373 \\
\hline LAV(3) & 0.012 & 0.016 \\
\hline $\begin{array}{c}\text { MHE(10-6 I,3) with } \\
\text { using "quadprog" }\end{array}$ & 0.069 & 0.101 \\
\hline $\begin{array}{c}\text { RMHE(10-6 I,3) } \\
\text { usith }\end{array}$ & 0.209 & 0.805 \\
\hline $\begin{array}{c}\text { MHE(10 }\left(10^{-6} I, 3\right)^{\text {with }} \\
\text { using ADMM }\end{array}$ & 0.024 & 0.037 \\
\hline $\begin{array}{c}\text { RMHE(10-6 I,3) } \\
\text { usith }\end{array}$ & 0.050 & 0.169 \\
\hline
\end{tabular}

\subsubsection{Simulations Based on Nonlinear Measurement Model}

In this subsection, the IEEE 14-bus and 30-bus systems with SCADA measurements are used to test the iRMHE algorithm.

For the IEEE 14-bus system, the sensors are placed as shown in Fig. 5.8, where 1 voltage magnitude, 7 pairs of real and reactive power injections and 17 pairs of real and reactive power flow measurements are considered. The pdf of the voltage and power injection measurement noise $\epsilon_{i}$ is given in (3.34) with $\sigma_{i}=0.01, i=1, \ldots, 15$, while the pdf of the power flow measurement noise is given in (3.35) with $\sigma_{i}=$ $0.01, i=16, \ldots, 49$. Bus 1 is chosen as the slack bus, and there are $n=27$ states in 
the state vector $x=\left[V_{1} \cdots V_{14} \theta_{2} \cdots \theta_{14}\right]^{T}$. The parameters of iRMHE algorithm in the IEEE 14-bus system are listed as follows:

- The initial estimate $\mu_{0}=\left[\mathbf{1}_{14}^{T}, \mathbf{0}_{13}^{T}\right]^{T}$.

- Covariance of noise: $Q=10^{-5} I_{27}, R=\operatorname{diag}\left(\sigma_{1}^{2}, \ldots, \sigma_{49}^{2}\right), P_{1 \mid 0}=10^{2} I_{27}$.

- Horizon length $N+1=3$.

- Constraints: $-0.30 \leq \hat{\theta}_{j} \leq 0.01,0.95 \leq \hat{V}_{j} \leq 1.1$, where $j=1, \ldots, 14$.

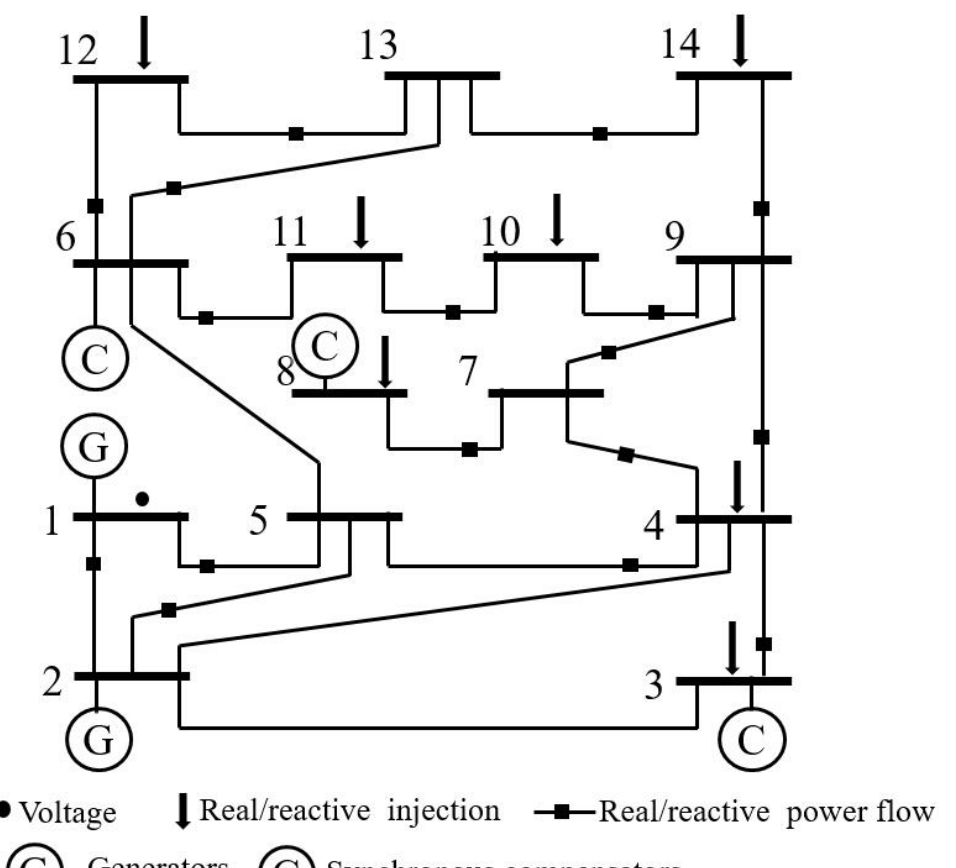

(G) Generators (C) Synchronous compensators

Figure 5.8: The IEEE 14-bus system with SCADA measurements.

For the IEEE 30-bus system, The measurement configuration (see Fig. 5.9) is the same as that in $[109,110]$, where 15 pairs of real and reactive power injections and 23 pairs of real and reactive power flow measurements are considered. The noise $\epsilon_{i}$ of the power injection measurements is associated with the pdf given in (3.34) with $\sigma_{i}=0.01, i=1, \ldots, 30$. The noise $\epsilon_{i}$ of the power flow measurements is associated with the pdf given in (3.35) with $\sigma_{i}=0.01, i=31, \ldots, 76$. Bus 1 is chosen as the 
slack bus and there are $n=59$ states in the state vector $x=\left[V_{1} \cdots V_{30} \theta_{2} \cdots \theta_{30}\right]^{T}$. The parameters of the iRMHE algorithm in the IEEE 30-bus system are listed as follows:

- The initial estimate $\mu_{0}=\left[\mathbf{1}_{30}^{T}, \mathbf{0}_{29}^{T}\right]^{T}$.

- Covariance: $Q=10^{-5} I_{59}, R=\operatorname{diag}\left(\sigma_{1}^{2}, \ldots, \sigma_{76}^{2}\right), P_{1 \mid 0}=10^{2} I_{59}$.

- Horizon length $N+1=3$.

- Constraints: $-0.35 \leq \hat{\theta}_{j} \leq 0.01,0.95 \leq \hat{V}_{j} \leq 1.1$, where $j=1, \ldots, 30$.

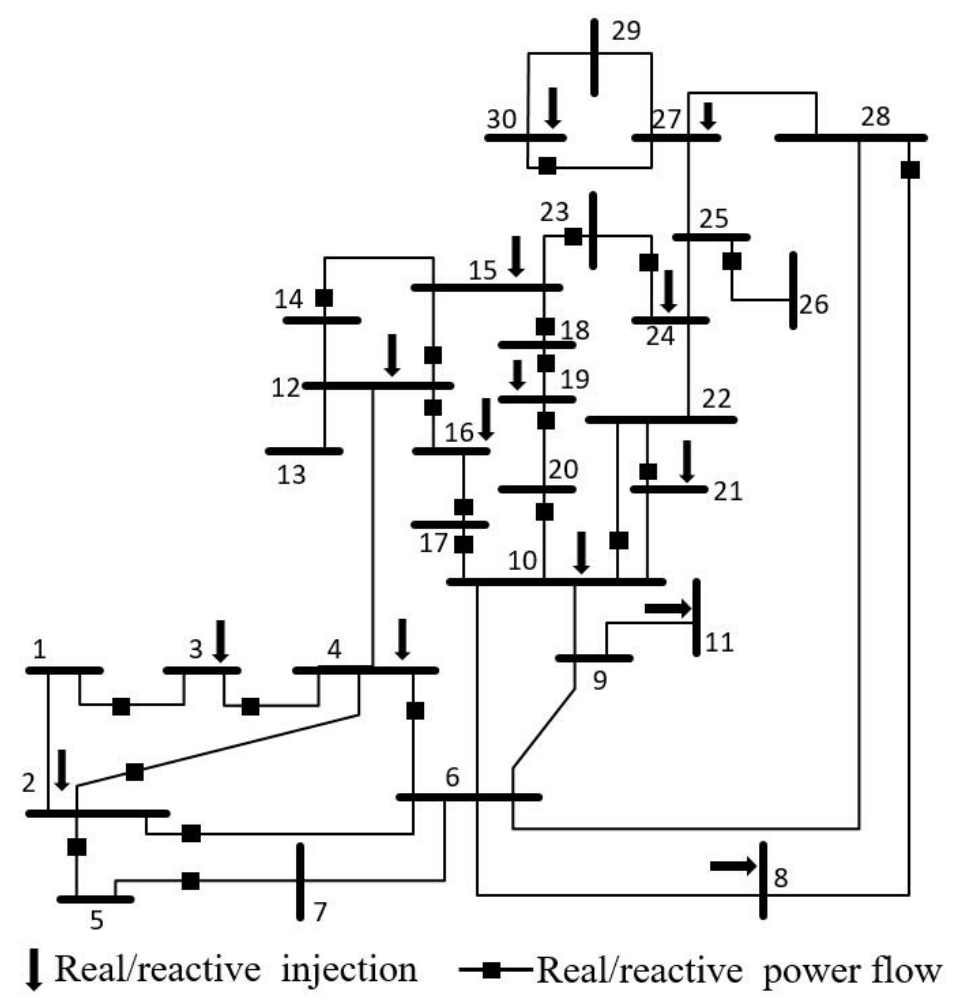

Figure 5.9: The IEEE 30-bus system with SCADA measurements.

The AMSE of different estimators in the IEEE 14-bus and 30-bus systems are given in Table 5.3, where the iRMHE $\left(Q=10^{-5} I, N+1=3\right)$ takes minimum AMSE, $3.1 \times 10^{-3}$ and $3.5 \times 10^{-3}$ for the IEEE 14 -bus and 30-bus systems respectively. The iMHE $\left(10^{-5} I, 3\right)$ with constraints uses a constant covariance matrix $R$ so its AMSE 
is larger than the one of $\mathrm{iRMHE}\left(10^{-5} I, 3\right)$ with constraints. The computation time of different estimators in the IEEE 14-bus and 30-bus systems is given in Table 5.4, where the maximum of the time step is 60 . The WLS with LNR takes least time in the IEEE 14-bus system, i.e, 0.028 s. However, the iRMHE with constraints does not spend much more time than other traditional robust estimators. For example, the iRMHE using ADMM takes $0.103 \mathrm{~s}$ while the MS estimator takes $0.102 \mathrm{~s}$ in the IEEE 14-bus system. Similar results can be drawn in the IEEE 30-bus system.

Table 5.3: The $\mathrm{AMSE}^{\natural}$ for different estimators with different parameters in the simulation examples.

\begin{tabular}{|c|c|c|c|c|}
\hline Model & \multicolumn{2}{|c|}{$\begin{array}{c}\text { IEEE 14-bus System, } \\
\text { with SCADA Measurements }\end{array}$} & \multicolumn{2}{|c|}{$\begin{array}{c}\text { IEEE 30-bus System } \\
\text { with SCADA Measurements }\end{array}$} \\
\hline $\begin{array}{c}\text { Measure- } \\
\text { ment } i\end{array}$ & $1, \ldots, 15$ & $16, \ldots, 49$ & $1, \ldots, 30$ & $31, \ldots, 76$ \\
\hline $\begin{array}{c}\text { Normal } \\
\text { Noise }\end{array}$ & $\begin{array}{c}0.97 \\
* N(0.01)\end{array}$ & $\begin{array}{c}0.97 \\
N(0.01)\end{array}$ & $\begin{array}{c}0.97 \\
N(0.01)\end{array}$ & $\begin{array}{c}0.97 \\
N(0.01)\end{array}$ \\
\hline Outliers & $\begin{array}{c}0.03 \\
N(0.1)\end{array}$ & $\begin{array}{c}0.03 \\
+U(0.1)\end{array}$ & $\begin{array}{c}0.03 \\
N(0.1)\end{array}$ & $\begin{array}{c}0.03 \\
U(0.1)\end{array}$ \\
\hline Estimator & $\ddagger(2.5,3.5,4.5)$ & $(3,4,5)$ & $(2.5,3.5,4.5)$ & $(3,4,5)$ \\
\hline${ }^{\sharp} \mathrm{WLS}(1)$ & \multicolumn{2}{|c|}{9.7} & \multicolumn{2}{|c|}{16.1} \\
\hline WLS with $\operatorname{LNR}(3)$ & \multicolumn{2}{|c|}{5.3} & \multicolumn{2}{|c|}{8.2} \\
\hline $\mathrm{QC}(3)$ & \multicolumn{2}{|c|}{5.2} & \multicolumn{2}{|c|}{7.6} \\
\hline $\mathrm{QL}(3)$ & \multicolumn{2}{|c|}{5.2} & \multicolumn{2}{|c|}{7.6} \\
\hline $\operatorname{SHGM}(3)$ & \multicolumn{2}{|c|}{5.2} & \multicolumn{2}{|c|}{7.7} \\
\hline $\operatorname{MS}(3)$ & \multicolumn{2}{|c|}{5.1} & \multicolumn{2}{|c|}{7.5} \\
\hline $\operatorname{SR}(3)$ & \multicolumn{2}{|c|}{5.2} & \multicolumn{2}{|c|}{7.6} \\
\hline $\mathcal{S}_{\mathrm{iMHE}}(\infty, 3)^{\text {without }}$ & \multicolumn{2}{|c|}{9.7} & \multicolumn{2}{|c|}{16.1} \\
\hline iRMHE $(\infty, 3)^{\text {without }}$ & \multicolumn{2}{|c|}{8.8} & \multicolumn{2}{|c|}{11.5} \\
\hline iMHE $\left(10^{-5} I, 3\right)^{\text {with }}$ & \multicolumn{2}{|c|}{3.8} & \multicolumn{2}{|c|}{4.8} \\
\hline iRMHE $\left(10^{-5} I, 3\right)^{\text {with }}$ & \multicolumn{2}{|c|}{3.1} & \multicolumn{2}{|c|}{3.5} \\
\hline Fig. & \multicolumn{2}{|c|}{5.11} & \multicolumn{2}{|c|}{5.12} \\
\hline
\end{tabular}

${ }^{\natural}$ AMSE unit: $\times 10^{-3}$.

${ }^{*} N\left(\sigma_{i}\right)=\frac{1}{\sqrt{2 \pi \sigma_{i}^{2}}} e^{-\frac{v_{i}^{2}}{2 \sigma_{i}^{2}}} ;{ }^{\dagger} U\left(\sigma_{i}\right)=\frac{1}{2 \sigma_{i}}$ for $-\sigma_{i} \leq v_{i} \leq \sigma_{i}$.

$\ddagger$ Parameter $a_{i}$ for QC, QL, SR and SHGM are the first term of the bracket $\left(a_{i}, b_{i}, r_{i}\right)$, while parameters $a_{i}, b_{i}, r_{i}$ for MS are given in the bracket of $\left(a_{i}, b_{i}, r_{i}\right)$.

\# Parameter $N+1$ for the traditional estimators are given in the bracket of $(N+1)$.

$\mathcal{S}$ Parameters $Q$ and $N+1$ for MHE and RMHE are given in the bracket of $(Q, N+1)$. The subscript without and with mean "without constraints" and "with constraints" respectively. 
Table 5.4: The computation time (per step) of different estimators based on nonlinear measurement model

\begin{tabular}{|c|c|c|}
\hline Model & $\begin{array}{c}\text { IEEE 14-bus system } \\
\text { with SCADA } \\
(\mathrm{s})\end{array}$ & $\begin{array}{c}\text { IEEE 30-bus system } \\
\text { with SCADA } \\
(\mathrm{s})\end{array}$ \\
\hline WLS with LNR (3) & 0.028 & 0.115 \\
\hline QC(3) & 0.057 & 0.126 \\
\hline QL(3) & 0.098 & 0.261 \\
\hline MS(3) & 0.102 & 0.267 \\
\hline SR(3) & 0.095 & 0.286 \\
\hline SHGM(3) & 0.136 & 0.334 \\
\hline $\begin{array}{c}\text { iRMHE(10 }\left(10^{-5} I, 3\right)^{\text {with }} \\
\text { using "quadprog" }\end{array}$ & 0.302 & 0.750 \\
\hline $\begin{array}{c}\left.\text { iRMHE(10 }{ }^{-5} I, 3\right)^{\text {with }} \\
\text { using ADMM }\end{array}$ & 0.103 & 0.371 \\
\hline
\end{tabular}

Take the simulation implemented in the IEEE 14-bus system as an example, and the MSE result is shown in Fig. 5.10. The MSE of iRMHE $\left(Q \rightarrow \infty, R^{q}=R\right)$ without constraints is equal to the result obtained by WLS. The MSE of iRMHE $\left(Q=10^{-5} I, N+1=3\right)$ with constraints in the IEEE 14 -bus and 30-bus systems are shown in Figs. 5.11 and 5.12 respectively, which shows that the MSE of iRMHE is lower than that of WLS and MS. The reason is that the iRMHE takes the constraints into account during the optimization process.

\subsection{Summary}

In this chapter, the constrained MHE in the linear system is firstly discussed. The re-weighted MHE (RMHE) reduces its sensitivity to the outliers by updating their error covariances in real time for robust PSSE. The influence of the outliers can be further mitigated. Applications are extended to the nonlinear measurement model and the proposed estimator is named as iRMHE. In order to accelerate the computational process, ADMM is applied to solve the quadratic programming problem arising from MHE. Simulation results illustrate the improvement on the state 


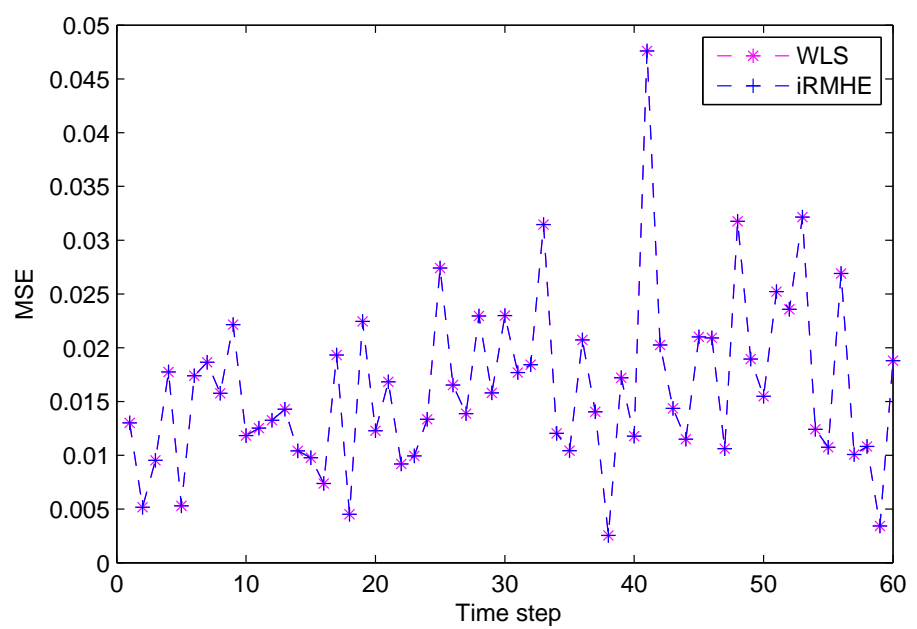

Figure 5.10: Mean square errors of WLS(1) and iRMHE $(Q \rightarrow \infty, N+1=1)$ without constraints in Example 3.

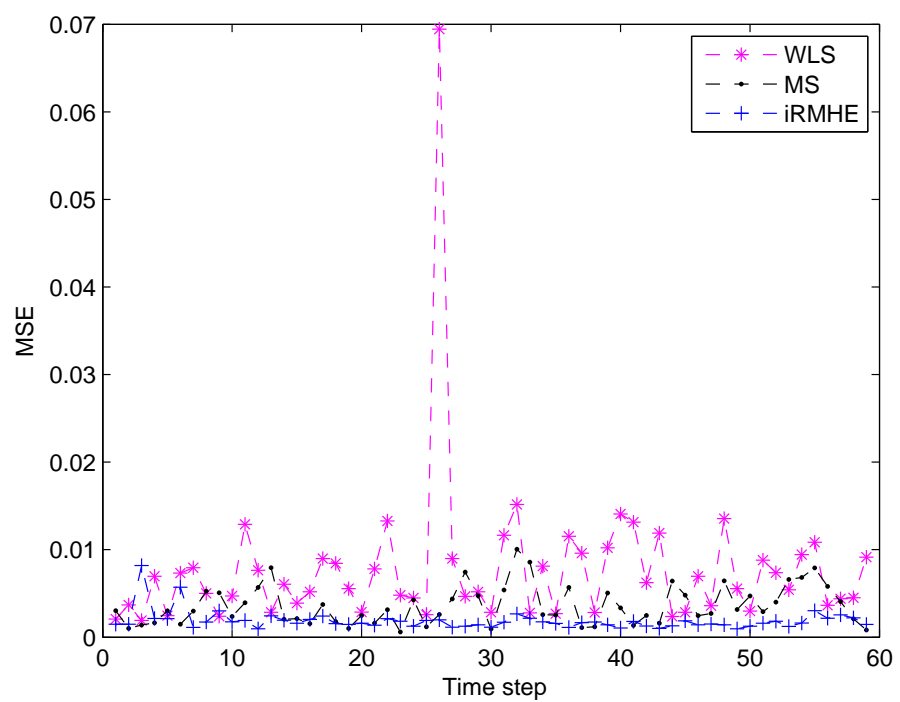

Figure 5.11: Mean square errors of WLS(1) and iRMHE $\left(Q=10^{-5} I, N+1=3\right)$ with constraints in the IEEE 14-bus system. 


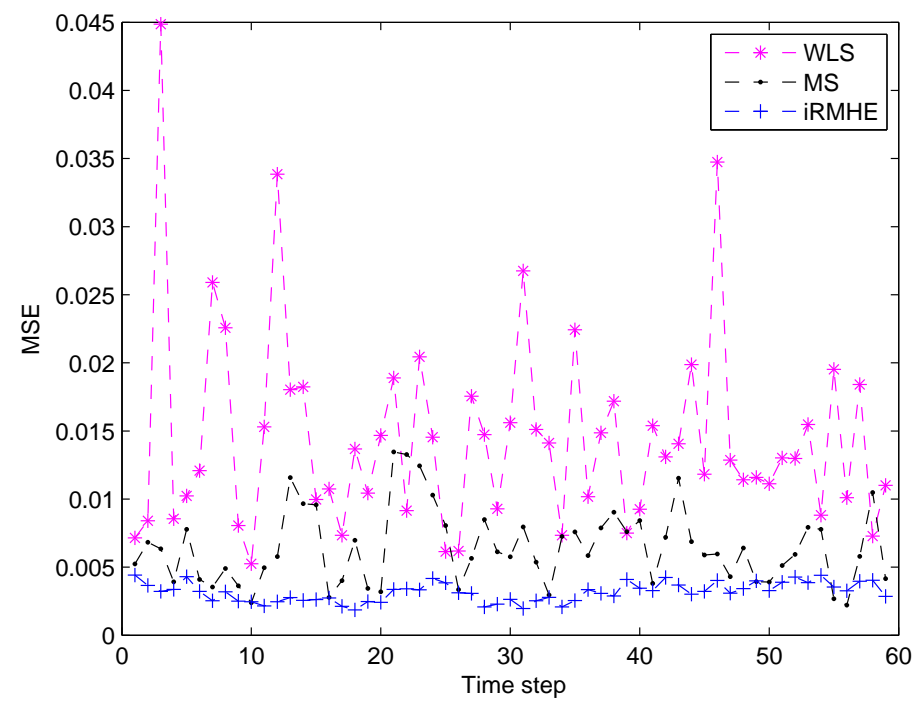

Figure 5.12: Mean square errors of WLS(1) and iRMHE $\left(Q=10^{-5} I, N+1=3\right)$ with constraints in the IEEE 30-bus system.

estimation after using the RMHE and iRMHE algorithms for linear and nonlinear measurement model, respectively. 


\section{Chapter 6}

\section{Distributed Moving Horizon}

\section{Estimation for Power Systems ${ }^{4}$}

In Chapter 5, the state estimation algorithms such as the RMHE and iRMHE are proposed in the framework of centralized setup, where all measurements are collected and sent to one centralized control center. Due to the enormous geographical span, the power system is separated into multiple small local areas and these local areas can only access some measurements. Distributed state estimation can solve such kind of problem. In this chapter both distributed MHE (DMHE) and iterated DMHE (iDMHE) [111] algorithms for linear and nonlinear measurement models are applied to PSSE respectively. Under the scheme of DMHE (or iDMHE), each local control area estimates the system states using only its own local measurements and measurements from its neighboring areas. Eventually, every local area will obtain the states of the whole system. Considering the fact that the scale of power system becomes large and it is usually separated into several local control areas installed with regional transmission organizations (RTOs). Each local control area can only

\footnotetext{
${ }^{4}$ Part of the materials in Chapter 6 is taken from paper "Tengpeng Chen, et al., Distributed Moving Horizon Estimation for Power Systems. IEEE Power 83 Energy Society General Meeting (PESGM), 1-5 (2015)."
} 
access its own local measurements. However, the advanced applications such as wide-area monitoring and control that require the system-wide state to be available to all the RTOs [79]. Therefore, the DMHE and iDMHE schemes are suitable for the applications mentioned above.

\subsection{Preliminaries}

The communication scheme among nodes is represented by a graph $\mathcal{G}=(\mathcal{V}, \mathbb{E})$. $\mathcal{V}$ denotes the nodes or vertices, where $\mathcal{V}=\{1,2, \cdots, \ell\} . \mathbb{E} \subseteq \mathcal{V} \times \mathcal{V}$ is the edges. The ordered pair $(j, i)$ belongs to $\mathbb{E}$ if node $i$ and node $j$ of $\mathcal{V}$ form an edge, i.e. node $i$ is connected to node $j$. The communication scheme is flexible and it may not necessarily represent a physical connection. In a graph that is formed by a communication scheme among nodes, $i$ is connected to $j$ if $j$ is able to transmit messages to $i$. The self edge $(i, i)$ is considered to belong to $\mathbb{E}$ as a node is capable of transmitting messages to itself. The neighboring set of a given node $i \in \mathcal{V}, \mathcal{N}_{i}$ is defined as

$$
\mathcal{N}_{i}:=\{j \in \mathcal{V} \mid(j, i) \in \mathbb{E}\}
$$

The neighboring set of node $i$ has cardinality of $\left|\mathcal{N}_{i}\right|$, which is equal to the edge number. Node $i$ is said to have degree $d_{i}=\left|\mathcal{N}_{i}\right|$.

\subsection{DMHE for Power Systems with Linear Mea- surement Model}

Under the scheme of DMHE, the power system is virtually separated into $\ell$ nonoverlapping local areas installed with corresponding local estimators. At every time 
step each local estimator solves a local optimization problem. The network of local control areas is required to be connected as defined in the graph theory [112-115], briefly presented in the last few paragraphs.

Denote $\mathcal{M}_{i}:=\{i\} \cup \mathcal{N}_{i}$ as the set of local area $i$ and neighbors $\mathcal{N}_{i}$, i.e., $\mathcal{M}_{i}=$ $\left\{i, j_{1}, \ldots, j_{\left|\mathcal{N}_{i}\right|}\right\}$. Define the regional measurement model of area $i$ as $[76]$

$$
\tilde{z}_{i, k}=\tilde{H}_{i} x_{i, k}+\tilde{v}_{i, k}, i=1, \ldots, \ell
$$

where

$$
\tilde{z}_{i}=\left[\begin{array}{c}
\breve{z}_{i, k} \\
\breve{z}_{j_{1}, k} \\
\vdots \\
\breve{z}_{j_{\left|\mathcal{N}_{i}\right|}, k}
\end{array}\right], \tilde{H}_{i}=\left[\begin{array}{c}
\breve{H}_{i} \\
\breve{H}_{j_{1}} \\
\vdots \\
\breve{H}_{j_{\left|\mathcal{N}_{i}\right|}}
\end{array}\right], \tilde{v}_{i, k}=\left[\begin{array}{c}
v_{i, k} \\
v_{j_{1}, k} \\
\vdots \\
v_{j_{\left|\mathcal{N}_{i}\right|}, k}
\end{array}\right]
$$

Note that $\breve{z}_{i, k} \in \mathbb{R}^{m_{i}}$ and $\breve{H}_{i} \in \mathbb{R}^{m_{i} \times n}$ are used to describe the measurements and the measurement matrix at control area $i . m_{i}$ is the number of measurements at local area $i$. It is assumed that each local area can collect the measurements recorded by itself and its neighboring sensors within a sampling interval. The global vectors are listed as

$$
\begin{aligned}
& z_{k}=\left[\breve{z}_{1, k}^{T} \cdots \breve{z}_{\ell, k}^{T}\right]^{T} \\
& v_{k}=\left[v_{1, k}^{T} \cdots v_{\ell, k}^{T}\right]^{T} \\
& H=\left[\breve{H}_{1}^{T} \cdots \breve{H}_{\ell}^{T}\right]^{T} .
\end{aligned}
$$

According to [76], the DMHE algorithm for the $i$-th area at time step $t$ determines 
the estimated results $\hat{x}_{i, t}$ by solving the minimization problem

$$
\Theta_{i, t}^{*}=\min _{x_{i, t-N},\left\{w_{i, k}\right\}_{k=t-N}^{t-1}} \Psi_{i, t}\left(x_{i, t-N},\left\{w_{i, k}\right\}\right)
$$

subject to

$$
\begin{aligned}
& x_{i, k+1}=A x_{i, k}+w_{i, k}, k=t-N, \ldots, t-1 \\
& \tilde{z}_{i, k}=\tilde{H}_{i, k} x_{i, k}+\tilde{v}_{i, k}, k=t-N, \ldots, t \\
& x_{i, k} \in \mathbb{X}
\end{aligned}
$$

and the objective function $\Psi_{i, t}$ is given by

$$
\begin{aligned}
\Psi_{i, t}\left(x_{i, t-N},\left\{w_{i, k}\right\}\right)= & \frac{1}{2} \sum_{k=t-N}^{t}\left\|\tilde{v}_{i, k}\right\|_{\tilde{R}_{i}^{-1}}^{2}+\frac{1}{2} \sum_{k=t-N}^{t-1}\left\|w_{i, k}\right\|_{Q^{-1}}^{2} \\
& +\Phi_{i, t-N}\left(x_{i, t-N}, \hat{\bar{x}}_{i, t-N \mid t-1}\right)
\end{aligned}
$$

where $\tilde{R}_{i}$ is diagonal matrix. The arrival cost $\Phi_{i, t-N}$ is given as

$$
\Phi_{i, t-N}=\frac{1}{2}\left\|x_{i, t-N}-\hat{\bar{x}}_{i, t-N \mid t-1}\right\|_{\Pi_{i, t-N \mid t-1}^{-1}}^{2}+\Theta_{i, t-1}^{*}
$$

in which the optimal cost $\Theta_{i, t-1}^{*}$ defined in (6.2) is a constant. Define the weighted average state estimation as

$$
\hat{\bar{x}}_{i, t-N \mid t-1}=\sum_{j=1}^{\ell} k_{i j} \hat{x}_{j, t-N \mid t-1}
$$

in which $k_{i j}$ is the entries of matrix $K$ describing the communication scheme, and $\sum_{j=1}^{\ell} k_{i j}=1, k_{i j} \geq 0$ if $j \in \mathcal{M}_{i}$; otherwise, $k_{i j}=0$. To update $\Pi_{i, t-N \mid t-1}$, firstly 
define the regional observability matrix $\tilde{O}_{i, N}$ as

$$
\tilde{O}_{i, N}=\left[\begin{array}{lll}
\left(\tilde{H}_{i}\right)^{T} & \left(\tilde{H}_{i} A\right)^{T} \ldots & \left(\tilde{H}_{i} A^{N-1}\right)^{T}
\end{array}\right]^{T}
$$

and

$$
\begin{aligned}
L_{i, N} & =\left[\begin{array}{cccc}
\mathbf{0} & \mathbf{0} & \ldots & \mathbf{0} \\
\tilde{H}_{i} & \mathbf{0} & \ldots & \mathbf{0} \\
\vdots & \vdots & \ddots & \vdots \\
\tilde{H}_{i} A^{N-2} & \tilde{H}_{i} A^{N-3} & \ldots & \tilde{H}_{i}
\end{array}\right] \\
\tilde{R}_{i, N} & =\operatorname{diag}\left(\tilde{R}_{i}, \ldots, \tilde{R}_{i}\right)_{N} \\
Q_{i, N-1} & =\operatorname{diag}(Q, \ldots, Q)_{N-1}
\end{aligned}
$$

Then the consensus weights are updated by

$$
\Pi_{i, t-N \mid t-1}=2 \sum_{j=1}^{\ell} k_{i j}^{2} \tilde{\Pi}_{j, t-N \mid t-1}
$$

where

$$
\begin{aligned}
\tilde{\Pi}_{i, t-N \mid t-1}=\quad A & \Pi_{i, t-N-1 \mid t-2}^{*} A^{T}+Q-A \Pi_{i, t-N-1 \mid t-2}^{*}\left(\tilde{O}_{i, N}\right)^{T} \times \\
& \times\left(\tilde{O}_{i, N} \Pi_{i, t-N-1 \mid t-2}^{*}\left(\tilde{O}_{i, N}\right)^{T}+\tilde{R}_{i, N}^{*}\right)^{-1} \times \\
& \times \tilde{O}_{i, N} \Pi_{i, t-N-1 \mid t-2}^{*} A^{T}
\end{aligned}
$$

in which

$$
\begin{aligned}
\Pi_{i, t-N-1 \mid t-2}^{*} & =\left[\left(\Pi_{i, t-N-1 \mid t-2}\right)^{-1}+\left(\tilde{H}_{i}\right)^{T}\left(\tilde{R}_{i}\right)^{-1} \tilde{H}_{i}\right]^{-1} \\
\tilde{R}_{i, N}^{*} & =\tilde{R}_{i, N}+L_{i, N} Q_{i, N-1}\left(L_{i, N}\right)^{T}
\end{aligned}
$$


Theorem 1: According to [76], there exists

$$
\Delta_{\mathrm{t}-\mathrm{N} \mid \mathrm{t}}=\mathrm{U} \Delta_{\mathrm{t}-\mathrm{N}-\mathbf{1} \mid \mathbf{t}-\mathbf{1}}+\varsigma_{\mathrm{t}}
$$

where $\boldsymbol{\Delta}$ is the estimation error and $\varsigma_{t}$ is a vanishing sequence. $\mathbf{U}=\mathbf{P}_{\mathbf{N O}} \mathbf{K} \mathbf{A} \mathbf{P}_{\mathbf{N O}}$, in which $\mathbf{P}_{N O}$ is an orthogonal projection matrix on the unobservable subspace [76], $\mathbf{A}=\operatorname{diag}(A, \ldots, A) \in \mathbb{R}^{n \ell \times n \ell}$ and $\mathbf{K}=K \otimes I_{n}$. In our simulations on the IEEE 14-bus and 30-bus systems, a suitable entry $k_{i j}$ of matrix $K$ is chosen to link to the communication scheme. Matrix $\mathbf{U}$ satisfies Theorem 1, so the convergent of state estimation is guaranteed.

The DMHE algorithm is summarized in the following pseudo-code:

DMHE Algorithm for Power Systems (for the $i$-th area):

1. Initialization: Set $\hat{x}_{i, 0}=\mu_{0}$. Choose the length $N$, matrices $Q, \tilde{R}_{i}$, and $\Pi_{0}$.

2. Main procedure:

Compute the state estimate $\hat{x}_{i, t}$.

while $t \geqslant 1$ do

(a) Whenever $t \leq N$, the estimation horizon $N$ is reduced to $\tilde{N}=t . \Pi_{i, 0 \mid t-1}^{-1}$ is computed from $\Pi_{0}$, solve (6.2) for $\hat{x}_{i, t-N},\left\{\hat{w}_{i, k}\right\}$ with $\Phi_{i, t-N}=\frac{1}{2} \| x_{i, 0}-$ $\hat{\bar{x}}_{i, 0 \mid t-1} \|_{\Pi_{i, 0 \mid t-1}^{-1}}^{2}$. Obtain $\hat{x}_{i, t}$ according to (5.11).

(b) When $t>N, \Pi_{i, t-N \mid t-1}^{-1}$ is computed from $\Pi_{t-N-1 \mid t-2}$. Solve (6.2) for $\hat{x}_{i, t-N},\left\{\hat{w}_{i, k}\right\}$ with $\Phi_{i, t-N}$ in (6.4). Obtain $\hat{x}_{i, t}$ according to (5.11).

\section{end while}




\subsection{DMHE for Power Systems with Nonlinear Mea- surement Model}

In this section the DMHE algorithm is presented for the nonlinear case. Considering the first-order term and neglecting the higher order terms in the Taylor series, $\breve{y}_{k}:=y_{k}-h\left(\hat{x}_{k-1}\right)+H_{k} \hat{x}_{k-1}$ is defined. For the nonlinear case the regional measurement of area $i$ is given as $\tilde{z}_{i, k}=\left[\breve{y}_{i, k} \breve{y}_{j_{1}, k} \ldots \breve{y}_{j_{\left|\mathcal{N}_{i}\right|}, k}\right]^{T}$.

Similar to the iMHE, in order to achieve a better approximation of $h(x)$, a secondary iteration is introduced into the DMHE algorithm. An improved approximation at time $t$ can be obtained by updating the linearization as the estimate of $x_{t}$ improves. This gives rise to an iteration within the estimation at time $t$, which will now be described. The resulting algorithm is called as 'iterated DMHE' or more compactly 'iDMHE'.

Let the solution at iteration index $q$ within the time step $t$ of area $i$ be denoted as $\hat{x}_{i, t}^{q}$. The objective function at iteration $q$ within time step $t$ in local area $i$ is given by

$$
\Theta_{i, t}^{*}=\min _{x_{i, t-N}^{q},\left\{w_{i, k}^{q}\right\}_{k=t-N}^{t-1}} \Psi_{i, t}\left(x_{i, t-N}^{q},\left\{w_{i, k}^{q}\right\}\right)
$$

subject to

$$
\begin{aligned}
& x_{i, k+1}^{q}=A x_{i, k}^{q}+w_{i, k}^{q}, k=t-N, \ldots, t-1 \\
& \tilde{z}_{i, k+1}=\tilde{H}_{i, k}^{q} x_{i, k}^{q}+\tilde{v}_{i, k}^{q}, k=t-N, \ldots, t \\
& x_{i, k}^{q} \in \mathbb{X}
\end{aligned}
$$


We just use the local vector $\hat{x}_{i, t}^{q-1}$ to linearize the Jacobian matrix $\bar{H}_{i}$ as follows:

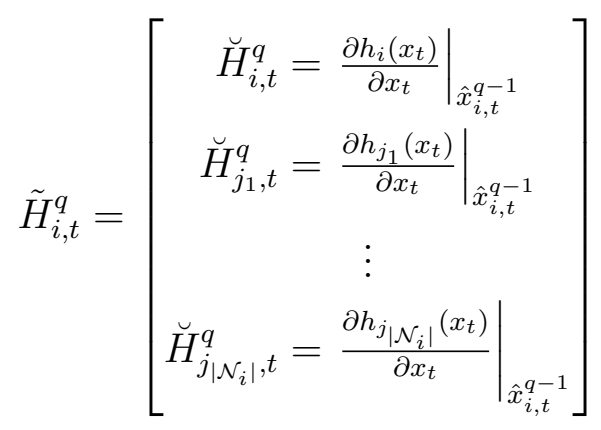

The local function $\Psi_{i, t}\left(x_{i, t-N}^{q},\left\{w_{i, k}^{q}\right\}\right)$ of iDMHE is given by

$$
\Psi_{i, t}\left(x_{i, t-N}^{q},\left\{w_{i, k}^{q}\right\}\right)=\frac{1}{2} \sum_{k=t-N}^{t}\left\|\tilde{v}_{i, k}^{q}\right\|_{\tilde{R}_{i}^{-1}}^{2}+\frac{1}{2} \sum_{k=t-N}^{t-1}\left\|w_{i, k}^{q}\right\|_{Q^{-1}}^{2}+\Phi_{i, t-N}
$$

For the nonlinear case, the following equation is used to update (6.6):

$$
\begin{aligned}
\tilde{\Pi}_{i, t-N \mid t-1}=A & \Pi_{i, t-N-1 \mid t-2}^{*} A^{T}+Q-A \Pi_{i, t-N-1 \mid t-2}^{*}\left(\tilde{O}_{i, N}\right)^{T} \times \\
& \times\left(\tilde{O}_{i, N} \Pi_{i, t-N-1 \mid t-2}^{*}\left(\tilde{O}_{i, N}\right)^{T}+\tilde{R}_{i, N}^{*}\right)^{-1} \times \\
& \times \tilde{O}_{i, N} \Pi_{i, t-N-1 \mid t-2}^{*} A^{T}
\end{aligned}
$$


in which

$$
\begin{aligned}
\Pi_{i, t-N-1 \mid t-2}^{*} & =\left[\left(\Pi_{i, t-N-1 \mid t-2}\right)^{-1}+\left(\tilde{H}_{i, t-N-1}\right)^{T}\left(\tilde{R}_{i}\right)^{-1} \tilde{H}_{i, t-N-1}\right]^{-1} \\
\tilde{R}_{i, N}^{*} & =\tilde{R}_{i, N}+L_{i, N} Q_{i, N-1}\left(L_{i, N}\right)^{T} \\
\tilde{O}_{i, N} & =\left[\begin{array}{cccc}
\left(\tilde{H}_{i, t-N+1}\right)^{T} & \left(\tilde{H}_{i, t-N+2} A\right)^{T} \ldots & \left(\tilde{H}_{i, t} A^{N-1}\right)^{T}
\end{array}\right]^{T} \\
L_{i, N} & =\left[\begin{array}{cccc}
\mathbf{0} & \mathbf{0} & \ldots & \mathbf{0} \\
\tilde{H}_{i, t-N+2} & \mathbf{0} & \ldots & \mathbf{0} \\
\vdots & \vdots & \ddots & \vdots \\
\tilde{H}_{i, t} A^{N-2} & \tilde{H}_{i, t} A^{N-3} & \ldots & \tilde{H}_{i, t}
\end{array}\right] \\
\tilde{R}_{i, N} & =\operatorname{diag}\left(\tilde{R}_{i}, \ldots, \tilde{R}_{i}\right)_{N} \\
Q_{i, N-1} & =\operatorname{diag}(Q, \ldots, Q)_{N-1}
\end{aligned}
$$

Initially, the estimation results obtained by each local area are different. The solution from each local area only converges to and reaches the consensus in some future time. Therefore, it is not necessary to iterate for a more accurate $H_{t}$ from time step 0 . In this work, the iteration process will only be started when the difference between the local control areas reaches some predefined value, i.e., $\left\|\hat{x}_{j, t}-\hat{x}_{i, t}\right\|=$ $\delta_{D M H E} \cdot \delta_{D M H E}$ is the chosen theshold. The iDMHE algorithm can be shown in the following pseudo-code:

iDMHE Algorithm for Power Systems (for the $i$-th area):

1. Initialization: Assign $\hat{x}_{i, 0}=\mu_{0}$. Choose the length $N$, weighting matrices $Q, \tilde{R}_{i}$, and $\Pi_{i, 0}$, the starting iteration threshold $\delta_{D M H E}$, and the iteration threshold $\delta_{i D M H E}$.

2. Main procedure:

Compute the state estimate $\hat{x}_{i, t}$. 
while $t \geqslant 1$ do

(a) Whenever $t \leq N$, the estimation horizon $N$ is reduced to $\tilde{N}=t . \Pi_{i, 0 \mid t-1}^{-1}$ is computed from $\Pi_{0}$. Solve (6.2) for $\hat{x}_{i, t-N},\left\{\hat{w}_{i, k}\right\}$ with $\Phi_{i, t-N}=\frac{1}{2} \| x_{i, 0}-$ $\hat{\bar{x}}_{i, 0 \mid t-1} \|_{\Pi_{i, 0 \mid t-1}^{-1}}^{2} ;$

(b) if $\left(\left\|\hat{x}_{j, t}-\hat{x}_{i, t}\right\|>\delta_{D M H E}, j \in N_{i}\right)$ do

i. Calculate $H_{i, t}$ using (6.9) for control area $i$, and $q=1$;

ii. Consensus weights update by (6.6) where $H_{i, k}$ is calculated at time $t-N+2$

iii. Calculate $\Phi_{i, t-N}$ using (6.4);

iv. Solve (6.2) for $\hat{x}_{i, t-N},\left\{\hat{w}_{i, k}\right\}$;

v. Obtain $\hat{x}_{i, t}$ according to (5.11);

(c) else

i. $q \leftarrow 1$;

ii. Set $\hat{x}_{i, t}^{q-1}=\hat{x}_{i, t-1}$;

while $\left(\left\|\hat{x}_{i, t}^{q}-\hat{x}_{i, t}^{q-1}\right\|>\delta_{i D M H E}\right)$ or $q \leqslant q_{\max }$ do

A. Calculate $H_{i, t}^{q}$ using (6.9);

B. Consensus weights update by (6.6) with matrix (6.11);

C. Calculate $\Phi_{i, t-N}$ using (6.4);

D. Solve (6.8) for $\hat{x}_{i, t-N}^{q},\left\{\hat{w}_{i, k}^{q}\right\}$;

E. Obtain $\hat{x}_{i, t}$ according to (5.11);

F. $q \leftarrow q+1$;

\section{end while}

\section{end if}

(d) $t \leftarrow t+1$; 
end while

\subsection{Simulation Results}

In this section, the performance of DMHE and iDMHE is evaluated through simulations studies conducted on linear and nonlinear measurement models. According to [86,91], $A$ is simplified as an identity matrix.

\subsubsection{Simulations Based on Linear Measurement Model}

In this subsection, MHE and DMHE algorithms are applied in the IEEE 14-bus and 30-bus systems with PMU measurements.

For the IEEE 14-bus system, the measurement noises are the same as those for the IEEE 14-bus system in Section 5.5.1. The true results are taken from [92], and we add small Gaussian noise following equation 5.2. The raw measurements are obtained via equation 5.1. The IEEE 14-bus system is partitioned into 4 nonoverlapping local areas, as shown in Fig. 6.1. The measurements in each local area are given in Table 6.1. The communication scheme among local areas is shown in Fig. 6.2, where the matrix $K$ is chosen according to the communication scheme and follows the rule " $\sum_{j=1}^{\ell} k_{i j}=1, k_{i j} \geq 0$ if $j \in \mathcal{M}_{i}$; otherwise, $k_{i j}=0$ ". The sets of each local area with its neighbors are listed as follows: $\mathcal{M}_{1}=\{1,4\}, \mathcal{M}_{2}=\{2,1\}$, $\mathcal{M}_{3}=\{3,2\}$ and $\mathcal{M}_{4}=\{4,1\}$. The initialization parameters of DMHE algorithm in the IEEE 14-bus system are as follows:

- For $i=1, \ldots, 4$, the covariance $\Pi_{i, 0}=10^{3} I_{28}$, and the initial estimate $\mu_{0}=$ $\left[\mathbf{1}_{14}^{T}, \mathbf{0}_{14}^{T}\right]^{T}$.

- Covariance of noise: $Q=10^{-6} I_{28}$. 
- Horizon length $N+1=3$.

- Constraints: $0.95 \leq V_{j}^{r} \leq 1.1,-0.35 \leq V_{j}^{i m} \leq 0.01$, where $j=1, \ldots, 14$.

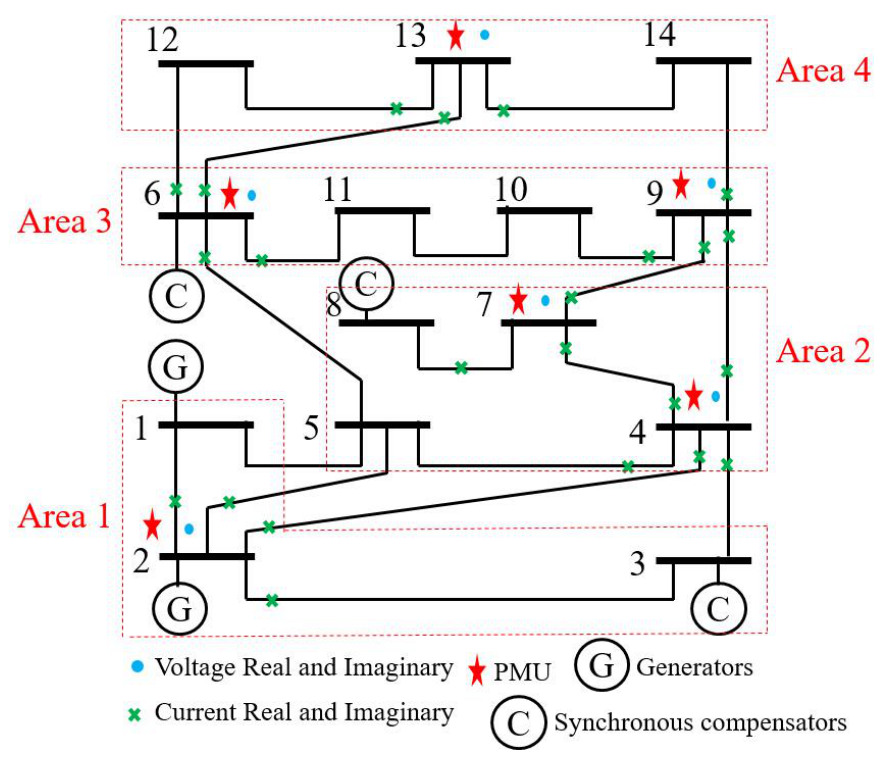

Figure 6.1: The IEEE 14-bus system installed with PMUs is separated into 4 local areas.

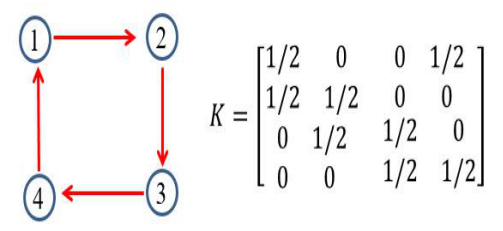

Figure 6.2: Communication scheme and matrix $K$.

When the IEEE 30-bus system installed with PMUs is separated into 4 local areas as shown in Fig. 6.3, the communication scheme is shown in Fig. 6.2. The measurement noises are the same as those for the IEEE 30-bus system in Section 5.5.1. The initialization parameters of DMHE algorithms on the IEEE 30-bus system are as follows:

- For $i=1, \ldots, 4$, the covariance $\Pi_{i, 0}=10^{3} I_{60}$, and the initial estimate $\mu_{0}=$ $\left[\mathbf{1}_{30}^{T}, \mathbf{0}_{30}^{T}\right]^{T}$. 
Table 6.1: Measurements (ignoring the subscript "mea") allocated for each area.

\begin{tabular}{|c|c|c|}
\hline Area & Numbers & Measurements type \\
\hline 1 & 10 & $\begin{aligned}\left\{V_{2}^{r}\right\},\{ & \left.V_{2}^{i m}\right\},\left\{I_{1,2}^{r}\right\},\left\{I_{2,3}^{r}\right\},\left\{I_{2,4}^{r}\right\},\left\{I_{2,5}^{r}\right\} \\
& \left\{I_{1,2}^{i m}\right\},\left\{I_{2,3}^{i m}\right\},\left\{I_{2,4}^{i m}\right\},\left\{I_{2,5}^{i m}\right\}\end{aligned}$ \\
\hline 2 & 20 & $\begin{array}{c}\left\{V_{4}^{r}\right\},\left\{V_{7}^{r}\right\},\left\{V_{4}^{i m}\right\},\left\{V_{7}^{i m}\right\}, \\
\left\{I_{4,2}^{r}\right\},\left\{I_{4,3}^{r}\right\},\left\{I_{4,5}^{r}\right\},\left\{I_{4,7}^{r}\right\},\left\{I_{4,9}^{r}\right\},\left\{I_{7,4}^{r}\right\},\left\{I_{7,8}^{r}\right\},\left\{I_{7,9}^{r}\right\} \\
\left\{I_{4,2}^{i m}\right\},\left\{I_{4,3}^{i m}\right\},\left\{I_{4,5}^{i m}\right\},\left\{I_{4,7}^{i m}\right\},\left\{I_{4,9}^{i m}\right\},\left\{I_{7,4}^{i m}\right\},\left\{I_{7,8}^{i m}\right\},\left\{I_{7,9}^{i m}\right\}\end{array}$ \\
\hline 3 & 20 & $\begin{array}{c}\left\{V_{6}^{r}\right\},\left\{V_{9}^{r}\right\},\left\{V_{6}^{i m}\right\},\left\{V_{9}^{i m}\right\}, \\
\left\{I_{6,5}^{r}\right\},\left\{I_{6,11}^{r}\right\},\left\{I_{6,12}^{r}\right\},\left\{I_{6,13}^{r}\right\},\left\{I_{9,4}^{r}\right\},\left\{I_{9,7}^{r}\right\},\left\{I_{9,10}^{r}\right\},\left\{I_{9,14}^{r}\right\} \\
\left\{I_{6,5}^{i m}\right\},\left\{I_{6,11}^{i m}\right\},\left\{I_{6,12}^{i m}\right\},\left\{I_{6,13}^{i m}\right\},\left\{I_{9,4}^{i m}\right\},\left\{I_{9,7}^{i m}\right\},\left\{I_{9,10}^{i m}\right\},\left\{I_{9,14}^{i m}\right\}\end{array}$ \\
\hline 4 & 8 & $\begin{aligned}\left\{V_{13}^{r}\right\},\left\{V_{13}^{i m}\right\},\left\{I_{13,6}^{r}\right\},\left\{I_{13,12}^{r}\right\},\left\{I_{13,14}^{r}\right\} \\
\\
\left\{I_{13,6}^{i m}\right\},\left\{I_{13,12}^{i m}\right\},\left\{I_{13,14}^{i m}\right\}\end{aligned}$ \\
\hline
\end{tabular}

- Covariance of noise: $Q=10^{-6} I_{60}$.

- Horizon length $N+1=3$.

- Constraints: $0.9 \leq V_{j}^{r} \leq 1.1,-0.4 \leq V_{j}^{i m} \leq 0.01$, where $j=1, \ldots, 30$.

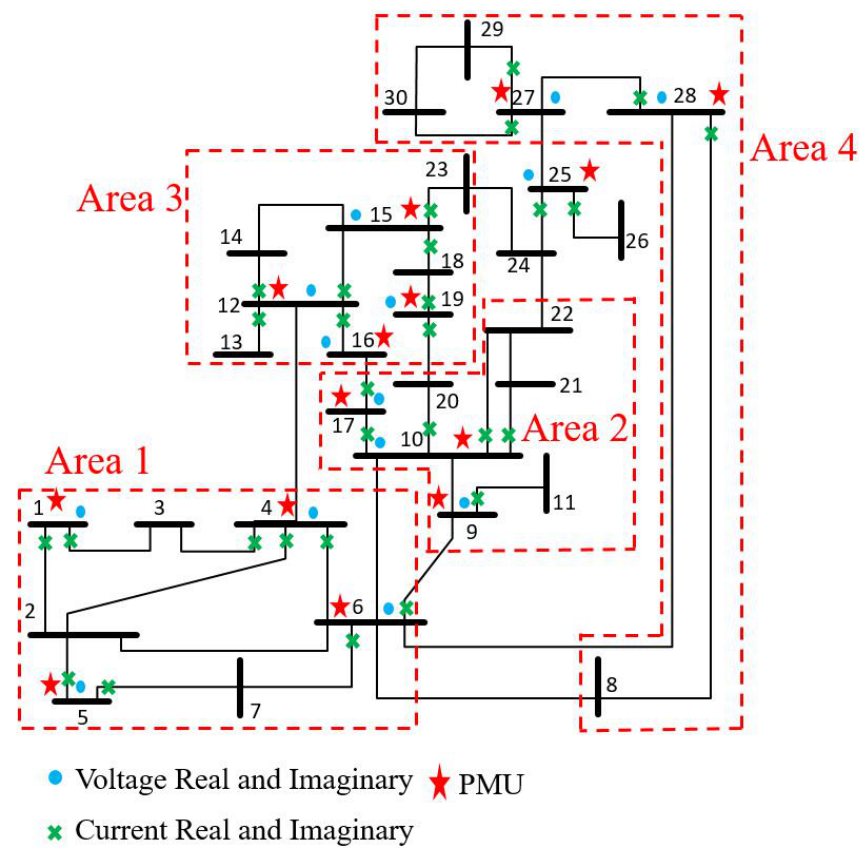

Figure 6.3: The IEEE 30-bus system installed with PMUs is separated into 4 local areas 
Denote the Mean Square Error (MSE) in area $i$ as

$$
M S E_{i, k}=\sqrt{\frac{1}{n}\left\|\hat{x}_{i, k}-x_{k}\right\|^{2}}, i=1, \ldots, 4
$$

Denote the Average of Mean Square Error (AMSE) for $k \in\left[t_{c}, t\right]$ to evaluate the estimation accuracy of DMHE:

$$
A M S E_{i}=\frac{1}{\left(t-t_{c}+1\right)} \sum_{k=t_{c}}^{t} \sqrt{\frac{1}{n}\left\|\hat{x}_{i, k}-x_{k}\right\|^{2}}
$$

where $t_{c}$ is the converging time step.

According to Figs. 6.4 and 6.5, it is verified that the estimated results of DMHE converge to the centralized results obtained from MHE. Convergence cannot be expected in fewer than 10 time-steps. Take the IEEE 14-bus system as an example, the real part of Bus 2 voltage phasor and the imaginary part of Bus 12 voltage phasor are shown in Figs. 6.6 and 6.7, respectively. The true results are taken from [92], and we add small Gaussian noise following 5.2. The raw measurements are obtained via 5.1. Both MHE and DMHE showed superior estimation performance in comparison to WLS without largest normalized residuals (LNR). The ADMM is used to solve the QP problems arising from DMHE, and the AMSE and the computation time of DMHE on the IEEE 14-bus and 30-bus systems are given in Table 6.2. It is clear that the AMSE of DMHE is almost the same as that of MHE. For the IEEE 14-bus system, the computation time of DMHE is $0.023 \mathrm{~s}$, while the centralized method MHE takes $0.024 \mathrm{~s}$. Under the scheme of DMHE, each local area solves an optimization problem to obtain the system-wide states. The time of DMHE is almost the same as the time that MHE takes. Similar conclusions can be drawn in the case of the IEEE 30-bus system. 


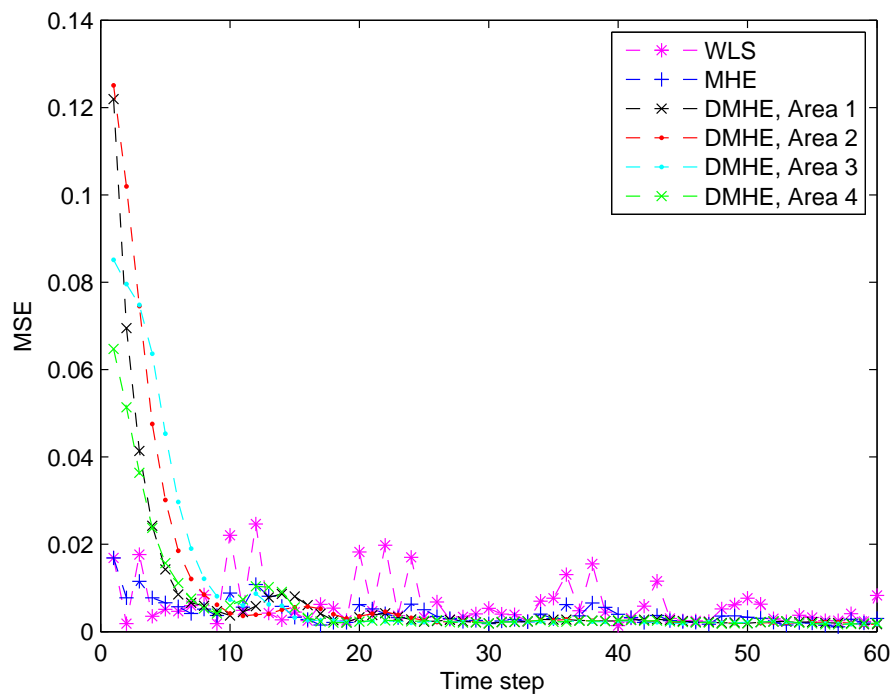

Figure 6.4: MSE of WLS, MHE and DMHE with constraints in the IEEE 14-bus system.

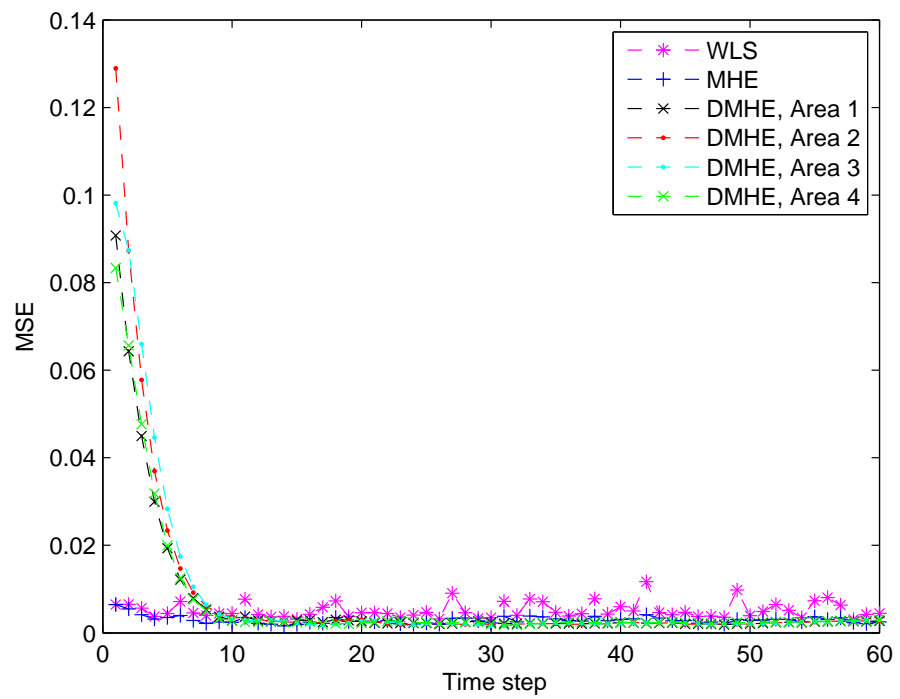

Figure 6.5: MSE of WLS, MHE and DMHE with constraints in the IEEE 30-bus system. 


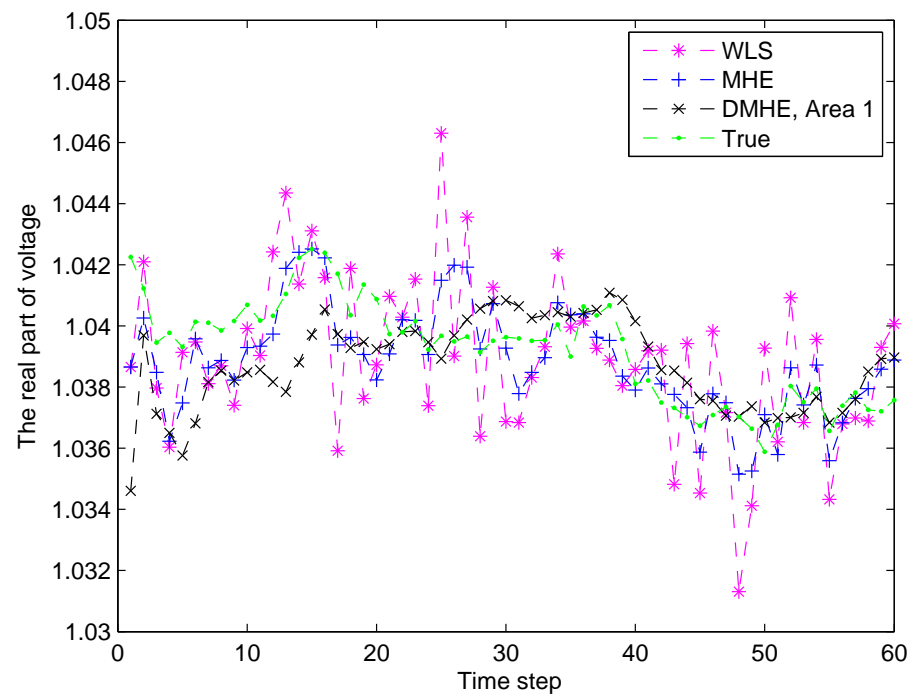

Figure 6.6: Real component of Bus 2 voltage phasor estimated by WLS, MHE and DMHE with constraints in the IEEE 14-bus system.

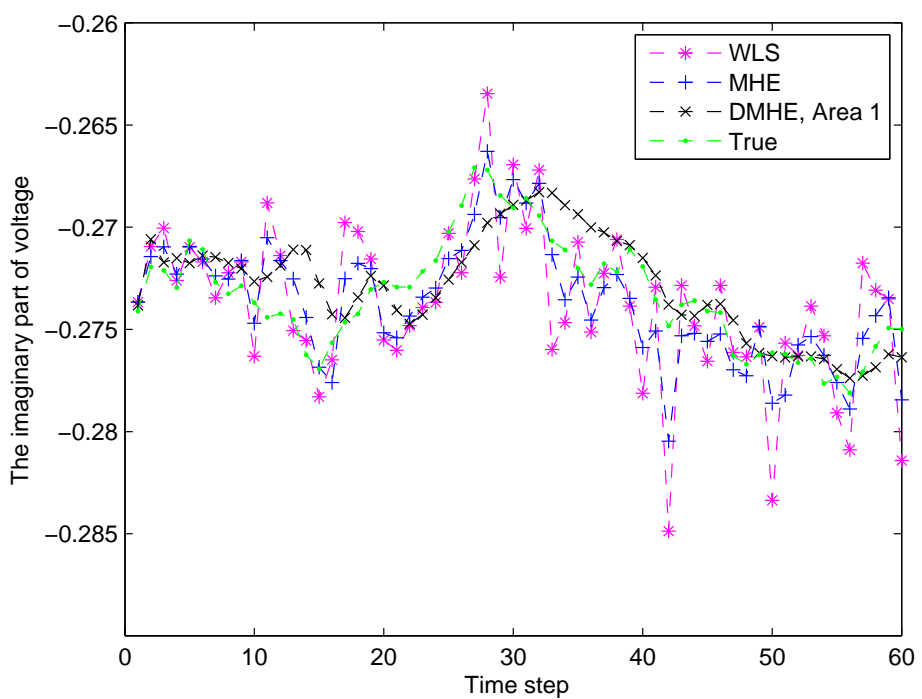

Figure 6.7: Imaginary component of Bus 12 voltage phasor estimated by WLS, MHE and DMHE with constraints in the IEEE 14-bus system. 
Table 6.2: The AMSE and computation time (per step) of MHE and DMHE with constraints based on linear measurement model.

\begin{tabular}{|c|c|c|c|c|}
\hline Model & \multicolumn{2}{|c|}{$\begin{array}{l}\text { IEEE 14-bus System, } \\
\text { with PMU Measurements }\end{array}$} & \multicolumn{2}{|c|}{$\begin{array}{c}\text { IEEE 30-bus System } \\
\text { with PMU Measurements }\end{array}$} \\
\hline Estimator & AMSE & $\begin{array}{c}\text { time } \\
(\mathrm{s})\end{array}$ & AMSE & $\begin{array}{c}\text { time } \\
(\mathrm{s})\end{array}$ \\
\hline MHE & 0.0017 & 0.024 & 0.0024 & 0.037 \\
\hline $\begin{array}{l}\text { DMHE (Area 1) } \\
\text { DMHE (Area 2) } \\
\text { DMHE (Area 3) } \\
\text { DMHE (Area 4) }\end{array}$ & 0.0018 & 0.023 & 0.0024 & 0.039 \\
\hline
\end{tabular}

\subsubsection{Simulations Based on Nonlinear Measurement Model}

In this subsection, a simulation study using the iDMHE algorithm is conducted on the IEEE 14-bus and 30-bus systems, where the measurements are collected from SCADA.

The IEEE 14-bus system is separated into 4 local areas, as shown in Fig. 6.8. The measurement noises follow those for the IEEE 14-bus system in Section 5.5.2. The communication scheme is shown in Fig. 6.9, where the matrix $K$ is chosen according to the communication scheme and follows the rule " $\sum_{j=1}^{\ell} k_{i j}=1, k_{i j} \geq 0$ if $j \in \mathcal{M}_{i}$; otherwise, $k_{i j}=0$ ". The parameters of iDMHE algorithm in the IEEE 14-bus system are listed as follows:

- For $i=1, \ldots, 4$, the covariance $\Pi_{i, 0}=10^{3} I_{27}$, and the initial estimate $\mu_{0}=$ $\left[\mathbf{1}_{14}^{T}, \mathbf{0}_{13}^{T}\right]^{T}$.

- Covariance of noise: $Q=10^{-5} I_{27}$.

- Horizon length $N+1=3$.

- Constraints: $-0.30 \leq \hat{\theta}_{j} \leq 0.01,0.95 \leq \hat{V}_{j} \leq 1.1$, where $j=1, \ldots, 14$.

- Threshold: $\delta_{i D M H E}=10^{-4}$, and $\delta_{D M H E}=10^{-1.5}$. 


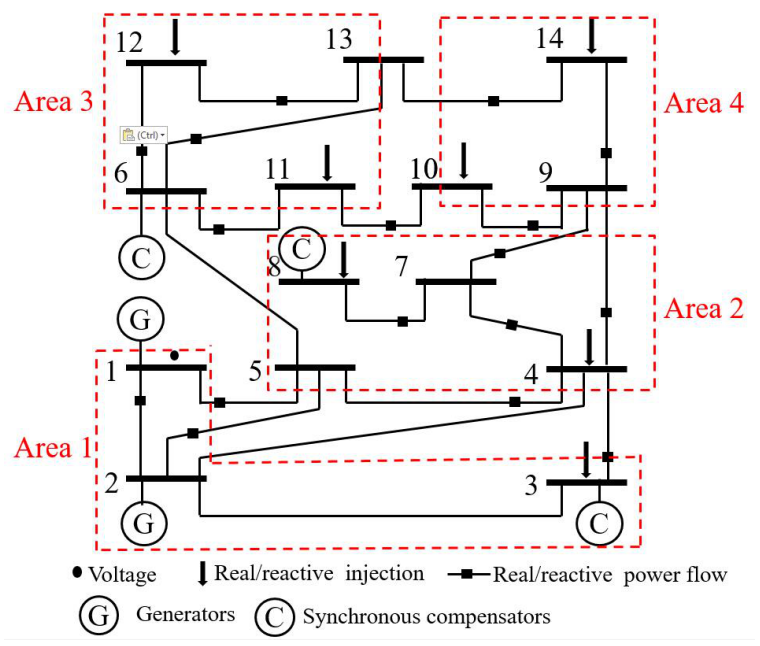

Figure 6.8: The IEEE 14-bus system with SCADA measurements.

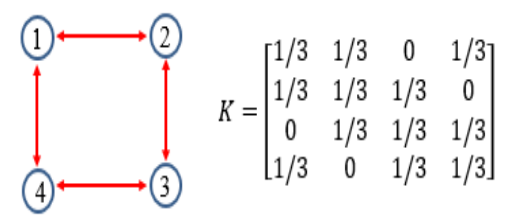

Figure 6.9: Communication scheme and matrix $K$.

The proposed algorithm is further implemented in the IEEE 30-bus system. The measurement noises are assumed to be the same as those in Section 5.5.2. The tested system is separated into 4 areas as shown in Fig. 6.10 and the communication scheme has been shown in Fig. 6.9. The parameters of the iDMHE algorithm in the IEEE 30-bus system are listed as follows:

- For $i=1, \ldots, 4$, the covariance $\Pi_{i, 0}=10^{3} I_{59}$, and the initial estimate $\mu_{0}=$ $\left[\mathbf{1}_{30}^{T}, \mathbf{0}_{29}^{T}\right]^{T}$.

- Covariance of noise: $Q=10^{-5} I_{59}$.

- Horizon length $N+1=3$.

- Constraints: $-0.35 \leq \hat{\theta}_{j} \leq 0.01,0.95 \leq \hat{V}_{j} \leq 1.1$, where $j=1, \ldots, 30$.

- Threshold: $\delta_{i D M H E}=10^{-4}$, and $\delta_{D M H E}=10^{-1.5}$. 


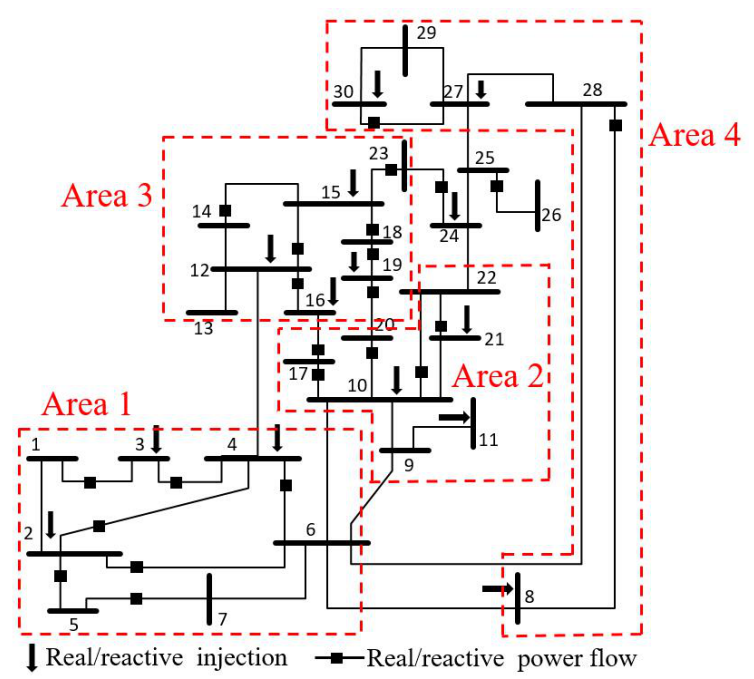

Figure 6.10: The IEEE 30-bus system with SCADA measurements.

The simulation results are shown in Figs. 6.11, 6.12, 6.13 and 6.14. It is clear that the iDMHE converges after 10 steps. The results obtained by WLS are quite far from the true states when outliers occur, while the iMHE/iDMHE with constraints weaken the influence caused by the outliers and their results are closer to the true state. The AMSE and computation time of iDMHE are given in Table 6.3. The AMSE of iDMHE is 0.0048 in the IEEE 30-bus system and it is similar to the centralized result. The time of the iDMHE $(0.368 \mathrm{~s})$ is also quite close to that of the iMHE, i.e., $0.364 \mathrm{~s}$.

Table 6.3: The AMSE and computation time of the iDMHE based on nonlinear measurement model.

\begin{tabular}{|c|c|c|c|c|}
\hline Model & \multicolumn{2}{|c|}{$\begin{array}{c}\text { IEEE 14-bus System } \\
\text { with SCADA Measurements }\end{array}$} & \multicolumn{2}{c|}{$\begin{array}{c}\text { IEEE 30-bus System } \\
\text { with SCADA Measurements }\end{array}$} \\
\hline Estimator & AMSE & $\begin{array}{c}\text { time } \\
(\mathrm{s})\end{array}$ & AMSE & $\begin{array}{c}\text { time } \\
(\mathrm{s})\end{array}$ \\
\hline iMHE & 0.0038 & 0.103 & 0.0048 & 0.364 \\
\hline $\begin{array}{l}\text { iDMHE (Area 1) } \\
\text { iDMHE (Area 2) } \\
\text { iDMHE (Area 3) } \\
\text { iDMHE (Area 4) }\end{array}$ & 0.0038 & 0.106 & 0.0048 & 0.368 \\
\hline
\end{tabular}




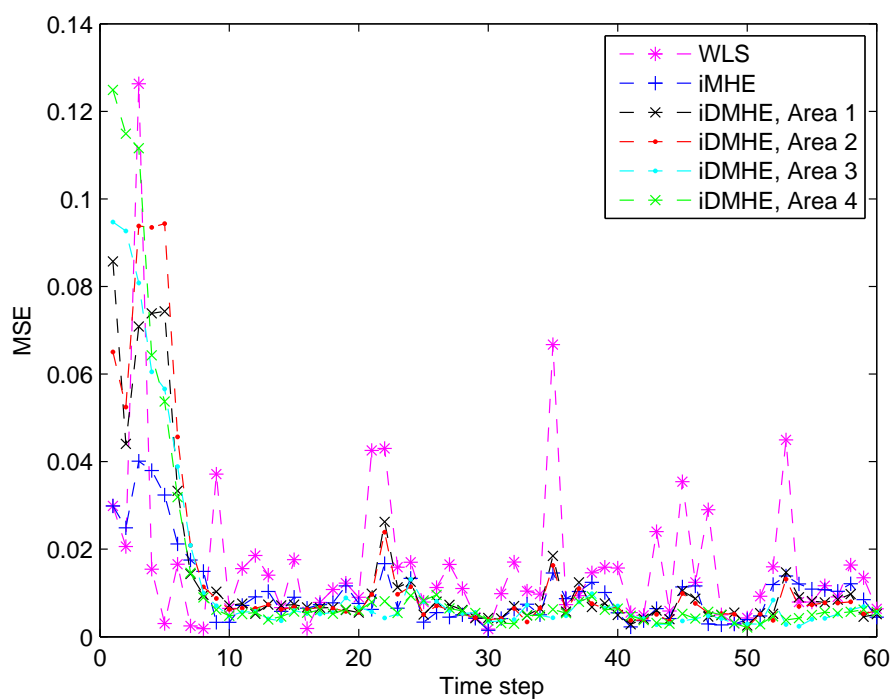

Figure 6.11: MSE of WLS, iMHE and iDMHE with constraints in the IEEE 14-bus system.

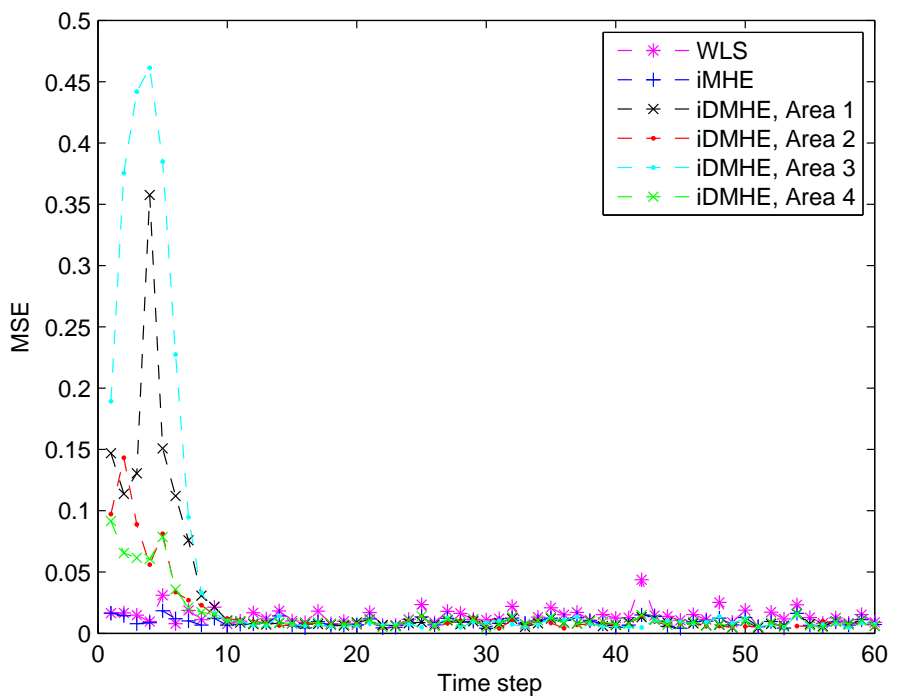

Figure 6.12: MSE of MHE, iMHE, DMHE and iDMHE with constraints in the IEEE 30-bus system. 


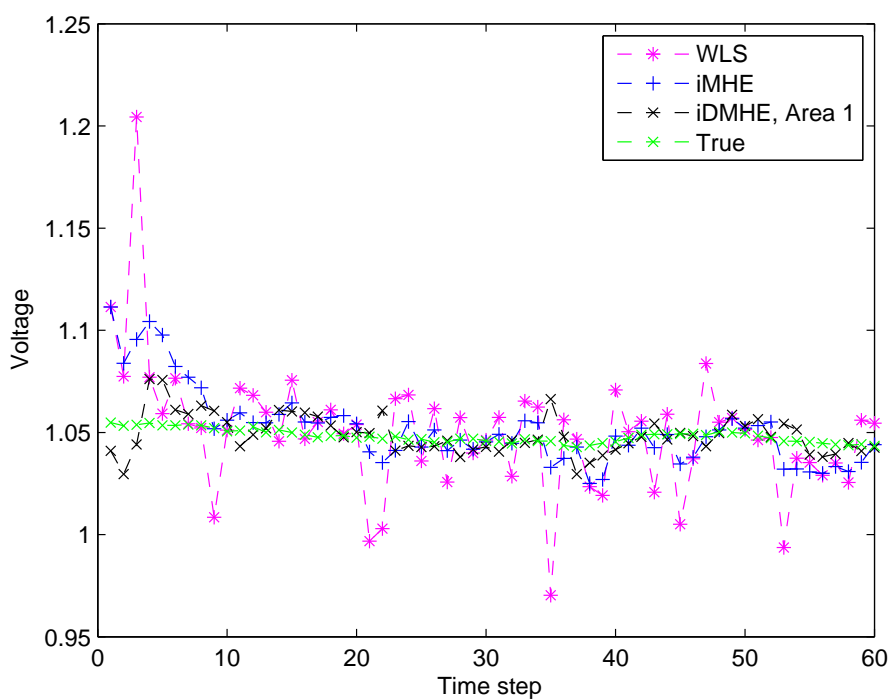

Figure 6.13: Bus 2 estimated voltage by WLS, iMHE and iDMHE with constraints in the IEEE 14-bus system.

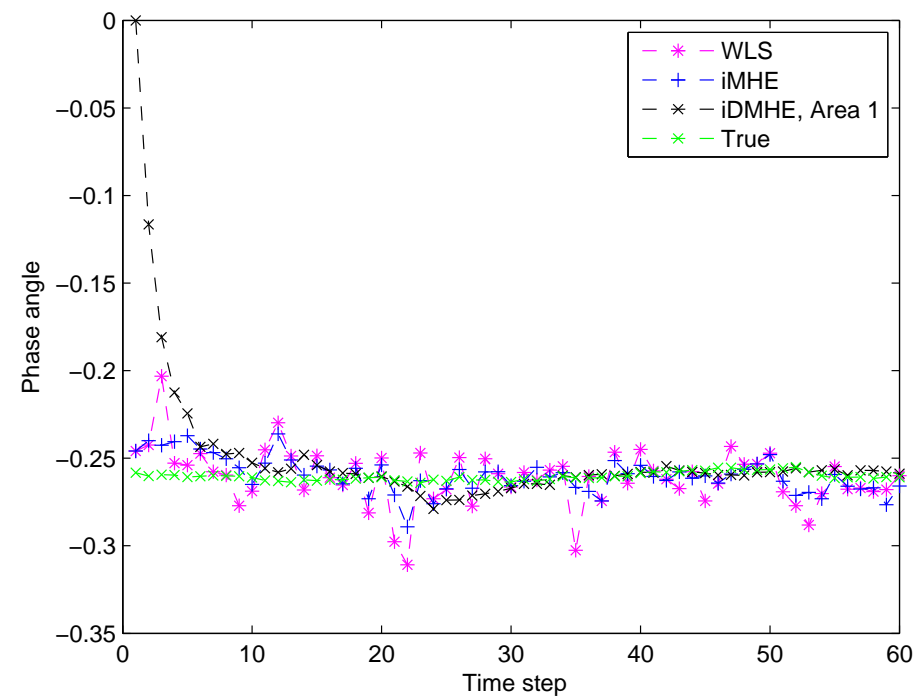

Figure 6.14: Bus 12 estimated phase angle by WLS, iMHE and iDMHE with constraints in the IEEE 14-bus system. 


\subsection{Summary}

In this chapter, the DMHE algorithm for PSSE is firstly presented. Each local control area estimates the states using only its own local measurements and measurements from its neighboring areas, and the estimates from its neighboring areas. Under the scheme of DMHE, each local area obtains the states of the whole system. Considering the fact that the scale of power system becomes large and it is usually separated into several local control areas installed with regional transmission organizations (RTOs). Each local control area can only access its own local measurements. However, the advanced applications such as wide-area monitoring and control that require the system-wide state to be available to all the RTOs [79]. Therefore, the DMHE is suitable in this case. For PSSE with the nonlinear measurement model, a secondary iteration is added to DMHE to highlight its accuracy and it is named as the iterated DMHE (iDMHE). Simulation results verify their good performance on PSSE. As a future work, the "re-weighted" process is to be added into DMHE and iDMHE to deal with outliers further. 


\section{Chapter 7}

\section{Re-weighted Partitioned Moving}

\section{Horizon Estimation for Power}

\section{Systems ${ }^{5}$}

Driven by the ongoing expansion of large-scale system monitoring, a distributed state estimation method named re-weighted partitioned MHE (RPMHE) is proposed in this chapter. The power systems are decomposed into several local areas with non-overlapping states. Information exchanges between neighboring areas so the communication load is alleviated compared with that of the centralized method. Under the scheme of DMHE, each local area solves the system-wide optimization problem and gets the whole system states. Here each local area solves a smaller optimization problem to estimate its own local states so the computation load in each area is smaller. The RPMHE is suitable for the power systems that each local areas only need its local own states $[116,117]$. The "re-weighted" process updates the covariances of measurement errors in real time to mitigate the influence of outliers

\footnotetext{
${ }^{5}$ Part of the materials in Chapter 7 is taken from paper "Tengpeng Chen, et al., Application of Partitioned-Based Moving Horizon Estimation in Power System State Estimation. Power and Energy Engineering Conference (APPEEC), IEEE PES Asia-Pacific, 1-5 (2015)."
} 
Chapter 7. Re-weighted Partitioned Moving Horizon Estimation for Power Systems $^{5}$

further.

\subsection{RPMHE for Power Systems with Linear Mea- surement Model}

In Chapter 5, the RMHE is implemented under the centralized setup, where all PMU measurements are collected and sent to some control center. However, it may be not feasible in practice for a large power network due to the communication bottleneck and regional disclosure policies [23]. The power system may be divided into multiple local areas (subsystems), where each area can only use its local measurements. In this section, on the basis of PMHE proposed in [28], a re-weighted PMHE approach is proposed for large-scale system monitoring, where each local area estimates its local states only based on the local measurements and local states information exchanging with its neighboring areas. The RPMHE is to implement RMHE in a distributed way.

\subsubsection{RPMHE Problem Formulation}

Let models (5.1) and (5.2) be partitioned into $\ell$ local control areas (subsystems) with non-overlapping states,

$$
\begin{aligned}
x_{k+1}^{[i]} & =A^{[i]} x_{k}^{[i]}+\tilde{A}^{[i]} x_{k}+w_{k}^{[i]} \\
z_{k}^{[i]} & =H^{[i]} x_{k}^{[i]}+\tilde{H}^{[i]} x_{k}+v_{k}^{[i]}
\end{aligned}
$$

where $x_{k}^{[i]} \in \mathbb{R}^{n_{i}}$ is the local state in area $i ; z_{k}^{[i]} \in \mathbb{R}^{m_{i}}$ represents the local measurements; $H^{[i]} \in \mathbb{R}^{m_{i} \times n_{i}}$ is the local measurement matrix; and $w_{k}^{[i]} \in \mathbb{R}^{n_{i}}$ and $v_{k}^{[i]} \in \mathbb{R}^{m_{i}}$ are the noises with covariances $Q^{[i]}$ and $R^{[i]}=\operatorname{diag}\left(\sigma_{1}^{2}, \ldots, \sigma_{m_{i}}^{2}\right)$, respectively. Ma- 
trices $\tilde{A}^{[i]}$ and $\tilde{H}^{[i]}$ have the structures in the form of

$$
\begin{aligned}
{\left[\left(\tilde{A}^{[1]}\right)^{T} \cdots\left(\tilde{A}^{[\ell]}\right)^{T}\right]^{T} } & =A-A^{*} \\
{\left[\left(\tilde{H}^{[1]}\right)^{T} \cdots\left(\tilde{H}^{[\ell]}\right)^{T}\right]^{T} } & =H-H^{*} .
\end{aligned}
$$

in which $A^{*}=\operatorname{diag}\left(A^{[1]}, \ldots, A^{[\ell]}\right)$ and $H^{*}=\operatorname{diag}\left(H^{[1]}, \ldots, H^{[\ell]}\right)$. The global vectors are $x_{k}=\left[\left(x_{k}^{[1]}\right)^{T} \ldots\left(x_{k}^{[\ell]}\right)^{T}\right]^{T}$ and $z_{k}=\left[\left(z_{k}^{[1]}\right)^{T} \ldots\left(z_{k}^{[\ell]}\right)^{T}\right]^{T}$.

The power system partitioning is based on the following assumption:

Assumption 1. The pair $\left(A^{[i]}, H^{[i]}\right)$ is locally observable, for $i=1, \ldots, \ell$.

Denote $\hat{x}_{k \mid t-1}^{[i]}$ as the estimation of $x_{k}^{[i]}$ performed at time step $t-1$. Approximate $\operatorname{Var}\left(x_{k \mid t-1}-\hat{x}_{k \mid t-1}\right)$ as $\Pi_{k \mid t-1}=\operatorname{diag}\left(\Pi_{k \mid t-1}^{[1]}, \ldots, \Pi_{k \mid t-1}^{[\ell]}\right)$. The constrained minimization problem RPMHE-i at time step $t$ is defined as [28]:

$$
\Theta_{t}^{*[i]}=\min _{\left(x_{t-N}^{[i]}\right)^{q},\left\{\left(w_{k}^{[i]}\right)^{q}\right\}_{k=t-N}^{t-1}} \Psi_{t}^{[i]}\left(\left(x_{t-N}^{[i]}\right)^{q},\left\{\left(w_{k}^{[i]}\right)^{q}\right\}\right)
$$

subject to

$$
\begin{aligned}
\left(x_{k+1}^{[i]}\right)^{q} & =A^{[i]}\left(x_{k}^{[i]}\right)^{q}+\left(w_{k}^{[i]}\right)^{q}, \quad k=t-N, \ldots, t-1 \\
z_{k}^{[i]} & =H^{[i]}\left(x_{k}^{[i]}\right)^{q}+\tilde{H}^{[i]} x_{k \mid t-1}+\left(v_{k}^{[i]}\right)^{q}, \quad k=t-N, \ldots, t \\
\left(x_{k}^{[i]}\right)^{q} & \in \mathbb{X}^{i}
\end{aligned}
$$

where $\mathbb{X}^{i}$ is the constraint set in local area $i$.

The local objective function in (7.5) is given by

$$
\begin{aligned}
\Psi_{t}^{[i]}\left(\left(x_{t-N}^{[i]}\right)^{q},\left(\left\{w_{k}^{[i]}\right\}\right)^{q}\right)= & \frac{1}{2} \sum_{k=t-N}^{t}\left\|v_{k}^{[i]}\right\|_{\left(\left(R_{k \mid t-1}^{[i]}\right)^{q}\right)^{-1}}^{2}+\frac{1}{2} \sum_{k=t-N}^{t-1}\left\|w_{k}^{[i]}\right\|_{\left(Q_{k \mid t-1}^{[i]}\right)^{-1}}^{2} \\
& +\Phi_{t-N}^{[i]}
\end{aligned}
$$


Chapter 7. Re-weighted Partitioned Moving Horizon Estimation for Power Systems $^{5}$

in which

$$
\begin{aligned}
\Phi_{t-N}^{[i]} & =\frac{1}{2}\left\|x_{t-N}^{[i]}-\hat{x}_{t-N \mid t-1}^{[i]}\right\|_{\left(\Pi_{t-N \mid t-1}^{[i]}\right)^{-1}}^{2} \\
Q_{k \mid t-1}^{[i]} & =Q^{[i]}+\tilde{A}^{[i]} \Pi_{k \mid t-1}\left(\tilde{A}^{[i]}\right)^{T} \\
\left(R_{k \mid t-1}^{[i]}\right)^{q} & =\left(R^{[i]}\right)^{q}+\tilde{H}^{[i]} \Pi_{k \mid t-1}\left(\tilde{H}^{[i]}\right)^{T}
\end{aligned}
$$

where $\tilde{H}$ is quite sparse and the second term in the right-hand side of (7.9) depends only on the neighboring areas. For RPMHE, the covariances of the measurement errors $j=1, \ldots, m_{i}$ in area $i$ are updated online according to the weights of MS estimator

$$
\begin{aligned}
&\left(R^{[i]}\right)^{q}= \operatorname{diag}\left(\left(R_{1, k}^{[i]}\right)^{q}, \ldots,\left(R_{m_{i}, k}^{[i]}\right)^{q}\right), \\
&\left(R_{j, k}^{[i]}\right)^{q}= \begin{cases}\sigma_{j}^{2} & \left|e_{j, k}\right| \leq a_{j} \sigma_{j} \\
\frac{\sigma_{j}\left|e_{j, k}\right|}{a_{j}} & a_{j} \sigma_{j}<\left|e_{j, k}\right| \leq b_{j} \sigma_{j} \\
\frac{\left(r_{j}-b_{j}\right) \sigma_{j}^{2}\left|e_{j, k}\right|}{a_{j}\left(r_{j} \sigma_{j}-\left|e_{j, k}\right|\right)} & b_{j} \sigma_{j}<\left|e_{j, k}\right| \leq r_{j} \sigma_{j} \\
+\infty & r_{j} \sigma_{j}<\left|e_{j, k}\right|\end{cases}
\end{aligned}
$$

where the measurement residual is given by

$$
e_{j, k}=z_{j, k}^{[i]}-\tilde{H}^{[i]} \hat{x}_{k \mid t-1}-H_{j}^{[i]}\left(\hat{x}_{k \mid t}^{[i]}\right)^{q-1}, k=t-N, \ldots, t
$$

\subsubsection{Update Matrices $\Pi_{t-N \mid t-1}^{[i]}$}

According to [28], denote the local observability matrix as

$$
\mathscr{O}_{N}^{[i]}=\left[\left(H^{[i]}\right)^{T} \ldots\left(H^{[i]}\left(A^{[i]}\right)^{N-1}\right)^{T}\right]^{T}
$$


and $\mathbf{0}$ as the matrix of zero elements. $\Pi_{t-N \mid t-1}^{[i]}$ is updated via:

$$
\begin{aligned}
\Pi_{t-N \mid t-1}^{[i]}= & A^{[i]} \bar{\Pi}_{t-N-1 \mid t-2}^{[i]}\left(A^{[i]}\right)^{T}+Q_{t-N-1 \mid t-2}^{[i]}-A^{[i]} \bar{\Pi}_{t-N-1 \mid t-2}^{[i]}\left(\mathscr{O}_{N}^{[i]}\right)^{T} \\
& \times\left(\mathscr{O}_{N}^{[i]} \bar{\Pi}_{t-N-1 \mid t-2}^{[i]}\left(\mathscr{O}_{N}^{[i]}\right)^{T}+\tilde{R}_{N \mid t-2}^{[i]}\right)^{-1} \mathscr{O}_{N}^{[i]} \bar{\Pi}_{t-N-1 \mid t-2}^{[i]}\left(A^{[i]}\right)^{T}
\end{aligned}
$$

where

$$
\begin{aligned}
\bar{\Pi}_{t-N-1 \mid t-2}^{[i]} & =\left(\left(\Pi_{t-N-1 \mid t-2}^{[i]}\right)^{-1}+\left(H^{[i]}\right)^{T}\left(R_{t-N-1 \mid t-2}^{[i]}\right)^{-1} H^{[i]}\right)^{-1} \\
\tilde{R}_{N \mid t-2}^{[i]} & =R_{N \mid t-2}^{[i]}+\mathscr{L}_{w, N}^{[i]} Q_{N-1 \mid t-2}^{[i]}\left(\mathscr{L}_{w, N}^{[i]}\right)^{T} \\
R_{N \mid t-2}^{[i]} & =\operatorname{diag}\left(R_{t-N \mid t-2}^{[i]}, \ldots, R_{t-1 \mid t-2}^{[i]}\right) \\
Q_{N-1 \mid t-2}^{[i]} & =\operatorname{diag}\left(Q_{t-N \mid t-2}^{[i]}, \ldots, Q_{t-2 \mid t-2}^{[i]}\right)
\end{aligned}
$$

$$
\mathscr{L}_{w, N}^{[i]}=\left[\begin{array}{cccc}
\mathbf{0} & \mathbf{0} & \ldots & \mathbf{0} \\
H^{[i]} & \mathbf{0} & \ldots & \mathbf{0} \\
\vdots & \vdots & \ddots & \vdots \\
H^{[i]}\left(A^{[i]}\right)^{N-2} & H^{[i]}\left(A^{[i]}\right)^{N-3} & \ldots & H^{[i]}
\end{array}\right]
$$

\subsubsection{RPMHE Convergence Properties}

According to [28], denote the matrix $\Xi$ as

$$
\Xi_{R P M H E}=M_{2}-M_{1}\left(\left(\mathscr{O}_{N+1}^{*}\right)^{T} \mathscr{O}_{N+1}^{*}\right)^{-1}\left(\mathscr{O}_{N+1}^{*}\right)^{T} \tilde{\mathscr{L}}_{N+1}
$$


Chapter 7. Re-weighted Partitioned Moving Horizon Estimation for Power Systems $^{5}$

where

$$
\begin{aligned}
\mathscr{O}_{N+1}^{*}= & \operatorname{diag}\left(\mathscr{O}_{N+1}^{[1]}, \ldots, \mathscr{O}_{N+1}^{[\ell]}\right) \\
\mathscr{O}_{N+1}^{[i]}= & {\left[\left(H^{[i]}\right)^{T} \ldots\left(H^{[i]}\left(A^{[i]}\right)^{N}\right)^{T}\right]^{T} } \\
\tilde{\mathscr{L}}_{N+1}= & {\left[\begin{array}{cccc}
\tilde{C} & \mathbf{0} & \ldots & \mathbf{0} \\
C^{*} \tilde{A} & \tilde{C} & \ldots & \mathbf{0} \\
\vdots & \vdots & \ddots & \vdots \\
C^{*}\left(A^{*}\right)^{N-1} \tilde{A} & C^{*}\left(A^{*}\right)^{N-2} \tilde{A} & \ldots & \tilde{C}
\end{array}\right] }
\end{aligned}
$$

$$
\begin{aligned}
M_{1}= & {\left[\left(A^{*}\right)^{T} \ldots\left(\left(A^{*}\right)^{N+1}\right)^{T}\right]^{T} } \\
M_{2}= & {\left[\begin{array}{cccc}
\tilde{A} & \mathbf{0} & \ldots & \mathbf{0} \\
\vdots & \vdots & \ddots & \vdots \\
\left(A^{*}\right)^{N-1} \tilde{A} & \left(A^{*}\right)^{N-2} \tilde{A} & \ldots & \mathbf{0} \\
\left(A^{*}\right)^{N} \tilde{A} & \left(A^{*}\right)^{N-1} \tilde{A} & \ldots & \tilde{A}
\end{array}\right] }
\end{aligned}
$$

If $\Pi_{t-N \mid t-1}^{[i]}$ are updated as the previous subsection and $\Xi_{R P M H E}$ is Schur, then PMHE is convergent [28]. The re-weighted process only works after PMHE converges, because the covariance $\left(R_{k \mid t-1}^{[i]}\right)^{q}$ calculated after the converging time $t_{R P M H E}$ is meaningful. The RPMHE algorithm for the power systems is listed in the following pseudo code:

RPMHE Algorithm for Power Systems (for the $i$-th area):

1. Initialization: Set $\hat{x}_{0}^{[i]}=\mu^{[i]}$, where $\mu^{[i]}$ is given. Choose the length $N$, the starting time to use re-weighted covariances $t_{R P M H E}$, and matrices $Q^{[i]}, R^{[i]}$, and $\Pi_{0}^{[i]}, i=1, \ldots, \ell$.

2. Main procedure: 
Compute the state estimation $\hat{x}_{t}^{[i]}$.

while new measurement exists do

(a) if $t \leq N$, the estimation horizon $N$ is reduced to $\tilde{N}=t$. Solve (7.5) with $\Phi_{t-N}^{[i]}=\frac{1}{2}\left\|x_{0}^{[i]}-\hat{x}_{0 \mid t-1}^{[i]}\right\|_{\left(\Pi_{0 \mid t-1}^{[i]}\right)^{-1}}^{2}$.

(b) else

i. if $t \leq t_{R P M H E}$, do
A. $\left(R^{[i]}\right)^{q}$ is set to a constant matrix $R^{[i]}$.
B. Solve (7.5) for $\left(\hat{x}_{t-N}^{[i]}\right)^{q},\left\{\left(\hat{w}_{k}^{[i]}\right)^{q}\right\}$;
C. Obtain $\hat{x}_{t}^{[i]}$ according to (5.11).

ii. else

iii. Set $q=0$;

iv. $\operatorname{Set}\left(\hat{x}_{t}^{[i]}\right)^{q}=\hat{x}_{t-1}^{[i]}$;

v. repeat
A. Set $q=q+1$
B. Calculate $\left(R_{k \mid t-1}^{[i]}\right)^{q}$ using (7.9).
C. Solve (7.5) for $\left(\hat{x}_{t-N}^{[i]}\right)^{q},\left\{\left(\hat{w}_{k}^{[i]}\right)^{q}\right\}$;
D. Obtain $\hat{x}_{t}^{[i]}$ according to (5.11).

vi. until $\left(\max \left(\left|\left(\hat{x}_{t}^{[i]}\right)^{q}-\left(\hat{x}_{t}^{[i]}\right)^{q-1}\right|\right) \leq \delta_{R P M H E}\right)$ or $q=q_{\max }$.

vii. end if

(c) end if

(d) $t \leftarrow t+1$;

end while 
Chapter 7. Re-weighted Partitioned Moving Horizon Estimation for Power Systems $^{5}$

\subsection{Simulation Results}

In this section the simulation studies are conducted on the IEEE 14-bus and 118bus systems to illustrate the effectiveness of RPMHE.

\subsubsection{Simulation on the IEEE 14-bus system}

As shown in Fig. 6.1, the IEEE 14-bus system is partitioned into 4 non-overlapping local control areas (subsystems), and the related communication scheme is shown in Fig. 7.1. Because there does not exist any physical line between area 1 and area 4, area 1 does not need to communicate with area 4 . The measurement noises are the same as those for the IEEE 14-bus system in Section 5.5.1. The measurements allocated for each area have been given in Table 6.1.

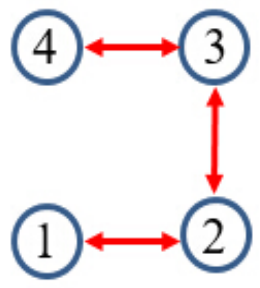

Figure 7.1: The communication scheme related to the partitioned areas.

Define the Mean Square Error (MSE) for the estimation results at time step $k$ in area $i$ as

$$
M S E_{k}^{[i]}=\sqrt{\frac{1}{n_{i}}\left\|\hat{x}_{k \mid k}^{[i]}-x_{k}^{[i]}\right\|^{2}}, i=1, \ldots, 4
$$

Denote the Average of Mean Square Error (AMSE) for $k \in\left[t_{c}, t\right]$ to evaluate the estimation accuracy:

$$
A M S E^{[i]}=\frac{1}{\left(t-t_{c}+1\right)} \sum_{k=t_{c}}^{t} \sqrt{\frac{1}{n_{i}}\left\|\hat{x}_{k \mid k}^{[i]}-x_{k}^{[i]}\right\|^{2}}
$$


where $t_{c}$ is the converging time step.

The initialization parameters of the RPMHE algorithm in the IEEE 14-bus system are listed as follows:

- The initial state vectors $x_{0}^{[1]}=x_{0}^{[4]}=1.5\left[\mathbf{1}_{3}^{T}, \mathbf{0}_{3}^{T}\right]^{T} ; x_{0}^{[2]}=x_{0}^{[3]}=1.5\left[\mathbf{1}_{4}^{T}, \mathbf{0}_{4}^{T}\right]^{T}$.

- The initial covariance matrices: $\Pi_{0}^{[1]}=\Pi_{0}^{[4]}=10^{3} I_{6}, \Pi_{0}^{[2]}=\Pi_{0}^{[3]}=10^{3} I_{8}$.

- The noise covariances: $Q_{0}^{[1]}=Q_{0}^{[4]}=10^{-6} I_{6}, Q_{0}^{[2]}=Q_{0}^{[3]}=10^{-6} I_{8} ; R_{0}^{[i]}=$ $\operatorname{diag}\left(\sigma_{1}^{2}, \ldots, \sigma_{m_{i}}^{2}\right), i=1, \ldots, 4$

- The horizon length: $N+1=3$.

- State constraints: $0.95 \leq V_{j}^{r} \leq 1.1,-0.35 \leq V_{j}^{i m} \leq 0.01$, where $j=1, \ldots, 14$.

These specific choices of parameters do not affect the generality of the RPMHE algorithm. In this simulation study, constraints on the state variables are taken into account for RMHE and RPMHE. The comparison of AMSE and average time obtained by RMHE and RPMHE are given in Table 7.1. The RPMHE takes 0.004 $\mathrm{s}$ while the RMHE takes $0.05 \mathrm{~s}$. The RPMHE is more than 10 times faster than the centralized MHE method. Fig. 7.2 shows the convergence of RPMHE with constraints and the converging time is around 22 time step. The results of the real component of Bus 2 and the imaginary component of Bus 14 provided by RPMHE are quite close to the true states, as shown in Figs. 7.3 and 7.4.

Table 7.1: The AMSE and computation time (per step) of RMHE and RPMHE with constraints in the IEEE 14-bus system.

\begin{tabular}{|c|c|c|}
\hline Estimator & AMSE & $\begin{array}{c}\text { time } \\
(\mathrm{s})\end{array}$ \\
\hline RMHE & 0.0014 & 0.050 \\
\hline RPMHE (Area 1) & 0.0015 & \\
RPMHE (Area 2) & 0.0014 & 0.004 \\
RPMHE (Area 3) & 0.0015 & \\
RPMHE (Area 4) & 0.0014 & \\
\hline
\end{tabular}


Chapter 7. Re-weighted Partitioned Moving Horizon Estimation for Power Systems $^{5}$

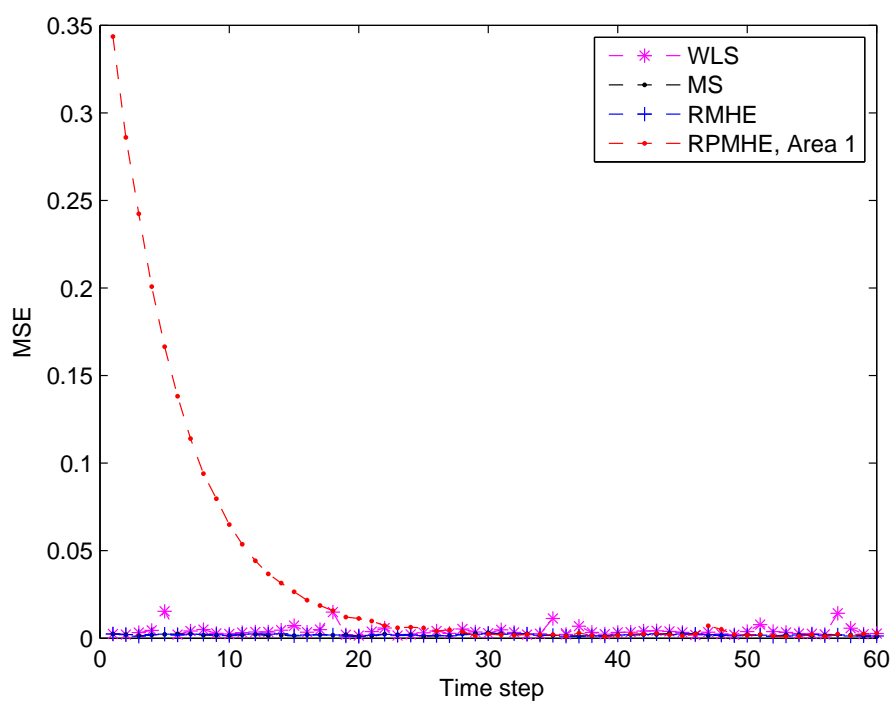

Figure 7.2: MSE of WLS, MS, RMHE and RPMHE with constraints in the IEEE 14-bus system.

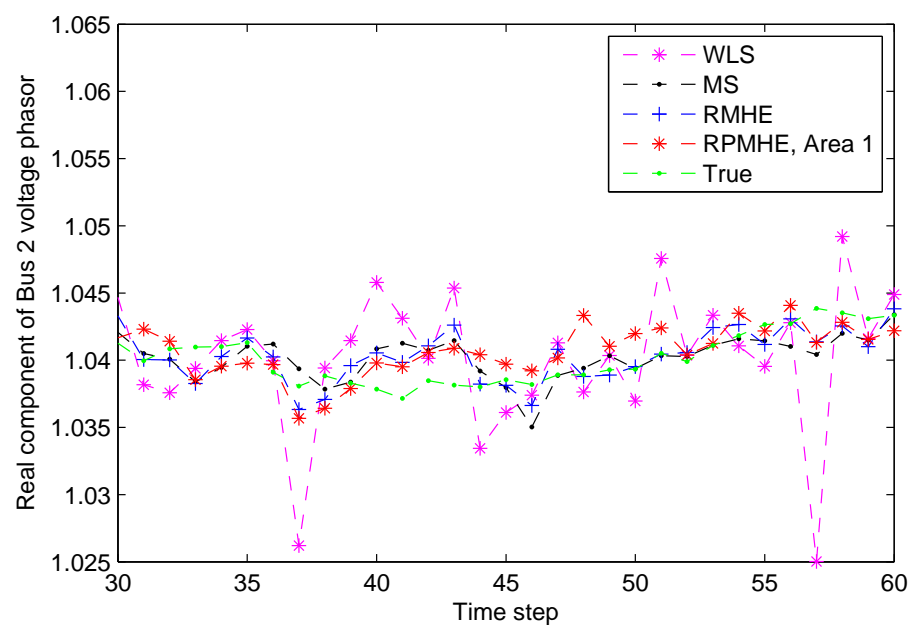

Figure 7.3: Real part of Bus 2 voltage phasor estimated by RPMHE with constraints (after converging) in the IEEE 14-bus system. 


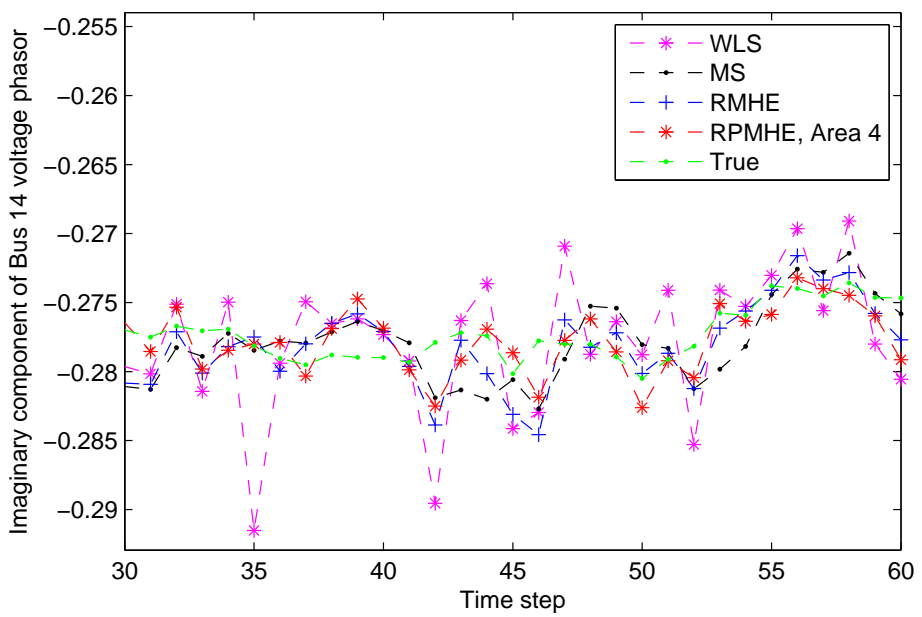

Figure 7.4: Imaginary part of Bus 14 voltage phasor estimated by RPMHE with constraints (after converging) in the IEEE 14-bus system.

\subsubsection{Simulation on the IEEE 118-bus system}

The proposed algorithm is applied in a lager-scale system. The IEEE 118-bus system is partitioned into 6 local areas (subsystems) as shown in Fig. 7.5. The relevant communication scheme is simplified as shown in Fig. 7.6. The PMUs are placed according to [91], a total number of 108 voltage measurements with the noise pdf in (3.34) with $\sigma_{i}=0.005, i=1, \ldots, 108$ and 366 current measurements with the noise pdf in (3.35) with $\sigma_{i}=0.01, i=109, \ldots, 474$ are considered. Area 1 has 46 measurements, Area 2 has 78 measurements, Area 3 has 76 measurements, both Area 4 and 5 have 110 measurements respectively, and Area 6 has 54 measurements. Every local area is locally observable. The imaginary part of Bus 69 voltage phasor is 0. The initialization parameters of RMHE algorithm in the IEEE 118-bus system are listed as follows:

- The initial state vectors $x_{0}^{[1]}=1.5\left[\mathbf{1}_{15}^{T}, \mathbf{0}_{15}^{T}\right]^{T} ; x_{0}^{[2]}=1.5\left[\mathbf{1}_{21}^{T}, \mathbf{0}_{21}^{T}\right]^{T} ; x_{0}^{[3]}=$ $1.5\left[\mathbf{1}_{17}^{T}, \mathbf{0}_{17}^{T}\right]^{T} ; x_{0}^{[4]}=1.5\left[\mathbf{1}_{25}^{T}, \mathbf{0}_{25}^{T}\right]^{T} ; x_{0}^{[5]}=1.5\left[\mathbf{1}_{27}^{T}, \mathbf{0}_{27}^{T}\right]^{T} ; x_{0}^{[6]}=1.5\left[\mathbf{1}_{13}^{T}, \mathbf{0}_{13}^{T}\right]^{T}$.

- The initial covariance matrices: $\Pi_{0}^{[1]}=10^{3} I_{30}, \Pi_{0}^{[2]}=10^{3} I_{42}, \Pi_{0}^{[3]}=10^{3} I_{34}$, 
Chapter 7. Re-weighted Partitioned Moving Horizon Estimation for Power Systems $^{5}$

$\Pi_{0}^{[4]}=10^{3} I_{50}, \Pi_{0}^{[5]}=10^{3} I_{54}, \Pi_{0}^{[6]}=10^{3} I_{26}$.

- The noise covariances: $Q_{0}^{[1]}=10^{-6} I_{30}, Q_{0}^{[2]}=10^{-6} I_{42}, Q_{0}^{[3]}=10^{-6} I_{34}, Q_{0}^{[4]}=$ $10^{-6} I_{50}, Q_{0}^{[5]}=10^{-6} I_{54}, Q_{0}^{[6]}=10^{-6} I_{26} \cdot R_{0}^{[i]}=\operatorname{diag}\left(\sigma_{1}^{2}, \ldots, \sigma_{m_{i}}^{2}\right), i=1, \ldots, 6$;

- The horizon length: $N+1=3$.

- State constraints: $0.85 \leq V_{j}^{r} \leq 1.1,-0.4 \leq V_{j}^{i m} \leq 0.4$, where $j=1, \ldots, 118$.

From Fig. 7.7, the RPMHE in the IEEE 118-bus system converges after 150 time steps. For simplicity, the time range to calculate AMSE for the IEEE 118-bus system is chosen from time step 250 to 300, and the AMSE and average time are given in Table 7.2. The RPMHE spends only $0.025 \mathrm{~s}$ and it takes much less time than RMHE (3.945 s).

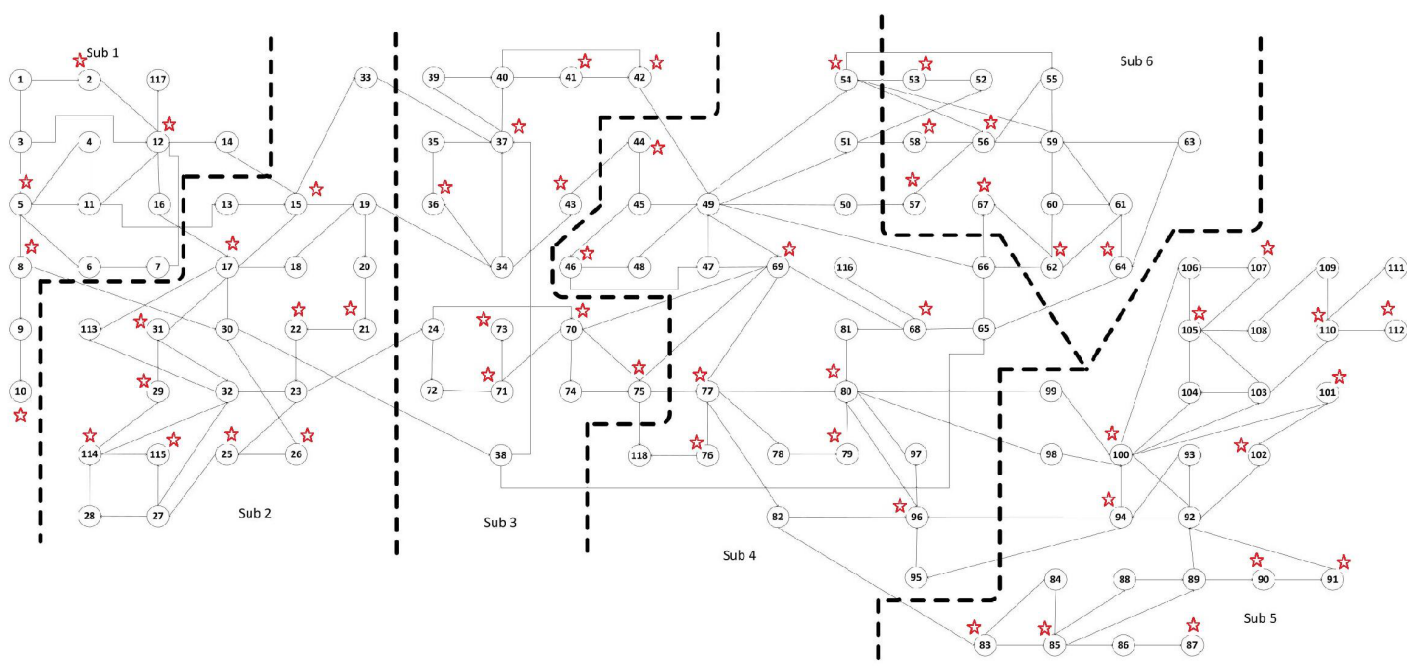

Figure 7.5: The IEEE 118-bus system with 6 partitioned local areas (subsystems) [5].

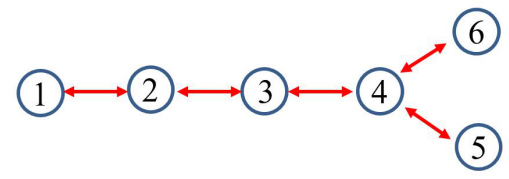

Figure 7.6: The communication scheme related to the partitioned IEEE 118-bus system. 


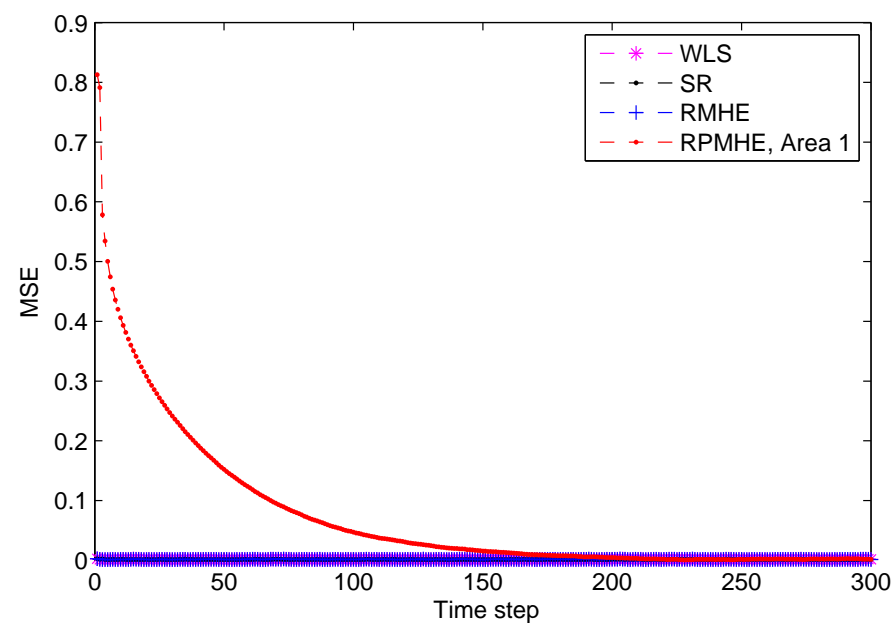

Figure 7.7: MSE of WLS, SR, RMHE and RPMHE with constraints in the IEEE 118-bus system.

Table 7.2: The comparison of AMSE and computation time (per step) of the RMHE and RPMHE with constraints in the IEEE 118-bus system.

\begin{tabular}{|c|c|c|}
\hline Estimator & AMSE & $\begin{array}{c}\text { time } \\
(\mathrm{s})\end{array}$ \\
\hline RMHE & 0.0009 & 3.945 \\
\hline \hline RPMHE (Area 1) & 0.0009 & \\
RPMHE (Area 2) & 0.0009 & \\
RPMHE (Area 3) & 0.0009 & 0.025 \\
RPMHE (Area 4) & 0.0009 & \\
RPMHE (Area 5) & 0.0009 & \\
RPMHE (Area 6) & 0.0009 & \\
\hline
\end{tabular}


Chapter 7. Re-weighted Partitioned Moving Horizon Estimation for Power Systems $^{5}$

\subsection{Summary}

Motivated by the large-scale system monitoring problem, a robust distributed state estimation algorithm named re-weighted partitioned MHE (RPMHE) is proposed for robust PSSE, where each local area (subsystem) solves a smaller optimization problem to estimate its own states so the computation load in each area (subsystem) becomes smaller. Simulations on the IEEE 14-bus and 118-bus systems have shown that the RPMHE spends less time than the centralized estimator. 


\section{Chapter 8}

\section{Conclusion and Future Research}

\subsection{Conclusion}

This thesis is dedicated to power system state estimation (PSSE). As a summary, the following aspects of PSSE were investigated:

1. An analytical equation is derived using influence function approximation to calculate the variance of the state estimate for traditional robust state estimators such as the Quadratic-Constant (QC), Quadratic-Linear (QL), SquareRoot (SR), Multiple-Segment (MS) and Schweppe-Huber Generalized-M (SHGM) estimator.The derived equation is useful: (1) It can be used to design an optimal state estimator. The computation load is reduced. The equation is $14,500(=14.5 / 0.001)$ times faster than Monte Carlo simulation. (2) It can be used to express the variance of a state estimate as a function of measurement variances enabling the selection of sensors for specified estimator precision.

2. A robust estimator based on the maximum likelihood criterion is proposed, in which the $t$-distribution is used to approximate the measurement noise instead of making the usual Gaussian assumption. Moreover, the influence function is employed to give an approximate solution to the maximum likelihood estima- 
tion problem. It makes the optimization problem to be solved iteratively and it is easy to be implemented in existing PSSE hardware and software. Due to a better approximation of the noise model, the proposed robust estimator could get better estimated results, compared with the results obtained from the traditional robust state estimators.

3. Constrained MHE is applied to PSSE. In order to reduce the sensitivity of outliers, a re-weighted MHE (RMHE) is proposed by updating the measurement error covariances in real time. Moreover, an iterated and re-weighted MHE (iRMHE) is proposed for power systems with nonlinear measurement model. In order to accelerate the performance of MHE, the Alternating Direction Method of Multipliers (ADMM) is adopted to solve the quadratic programming (QP) problem arising from MHE. Driven by the ongoing expansion of large-scale system monitoring, two distributed state estimation methods, namely distributed MHE (DMHE) and re-weighted partitioned MHE (RPMHE), are implemented in power systems.

\subsection{Recommendation for Future Research}

Although this research focused on the robust PSSE and distributed state estimation methods for power systems, the the works do not end here. The following aspects will be studied further:

1. Both centralized and distributed MHE for power system state estimation have been presented based on measurements from SCADA or PMUs. Due to the hardware limitations, the communication time between different local areas (or subsystems) or the communication failure are not considered in the current work. To make it more practical, relevant experiment would be conducted in future work. 
2. This thesis only focuses on the transmission system. The transmission system connects major generating stations and main load centers and it is the backbone of the modern power system. In fact the power network is typically classified into three main subsystems depending on different levels of voltage (from high to low). These three subsystems are transmission, subtransmission and distribution. In recent years scholars pay more attention to the state estimation based on distribution system, due to the development of micro-PMUs. The relevant works presented in this thesis would be extended to the distribution system.

3. The measurements presented in this thesis are collected only from SCADA or PMUs, but not from both of them at the same time. As evident from many publications on phasor measurements, the PMUs are expected to populate power systems in large numbers in a few short years $[4,33,91,118]$. This thesis also investigates the potential use of the PMUs. However, the current monitoring meters are gradually replaced with PMUs, but not to be done at the same time. Hence there exists a transition period when both measurements collected from SCADA and the ones obtained from PMUs are used in the same power system. The proposed approaches in this thesis are not based on the hybrid of measurement model. It would be interesting to apply the proposed algorithms to the hybrid system. 


\section{AUTHOR'S PUBLICATIONS}

1. Weng Khuen Ho, Tengpeng Chen*(corresponding author), Keck Voon Ling, and Lu Sun. "Variance Analysis of Robust State Estimation in Power System using Influence Function", International Journal of Electrical Power $\&$ Energy Systems, 92: 53-62 (2017).

2. Tengpeng Chen. "Robust State Estimation for Power Systems via Moving Horizon Strategy". Sustainable Energy, Grids and Networks, 10: 46-54 (2017).

3. Tengpeng Chen, Lu Sun, Keck Voon Ling, and Weng Khuen Ho. "Robust Power System State Estimation Using t-Distribution Noise Model". IEEE Transactions on Smart Grid, under review, 2017.

4. Lu Sun, Tengpeng Chen*(corresponding author), Xuebing Chen, Weng Khuen Ho, Keck Voon Ling, King Jet Tseng, and Gehan Amaratunga. "Optimum Placement of Phasor Measurement Unit in Power System", IEEE Transactions on Power Systems, under review, April, 2017.

5. Lu Sun, Tengpeng Chen, Weng Khuen Ho, and Keck Voon Ling. "Influence Function Analysis of LAV Dynamic State Estimation in Smart Grids". To be submitted to IEEE Transactions on Power Systems.

6. Tengpeng Chen, and Keck Voon Ling. "Robust Distributed State Estimation for Large-Scale Power Systems Using Moving Horizon Strategy". in preparation. 
7. Tengpeng Chen, Tri Tran, Dexiang Zhou, Weng Khuen Ho, and Keck Voon Ling. "Iterated Moving Horizon Estimation for Power Systems". International Conference on Control, Automation and Information Sciences (ICCAIS), 81$86(2014)$.

8. Tengpeng Chen, Dexiang Zhou, Tri Tran, C. Kastner, K.-V Ling, K.-J. Tseng, and J.M. Maciejowski. "Distributed Moving Horizon Estimation for Power Systems". IEEE Power \& Energy Society General Meeting (PESGM), 1-5 (2015). (One of the Best Conference Papers)

9. Tengpeng Chen, Ashok Krishnan, and Tri Tran. "Application of PartitionedBased Moving Horizon Estimation in Power System State Estimation". Power and Energy Engineering Conference (APPEEC), IEEE PES Asia-Pacific, 1-5 (2015).

10. Tri Tran, and Tengpeng Chen. "Dissipativity Constraint for Distributed Power System State Estimation". International Conference on Control, Automation and Information Sciences (ICCAIS), 360-365 (2015).

11. Xuebing Chen, Tengpeng Chen, King Jet Tseng, Yuhao Sun, and Gehan Amaratunga. "Customized Optimal micro-PMU Placement Method for Distribution Networks". Power and Energy Engineering Conference (APPEEC), IEEE PES Asia-Pacific, 135-140 (2016).

12. Xuebing Chen, Tengpeng Chen, King Jet Tseng, Yuhao Sun, and Gehan Amaratunga. "Hybrid Approach Based on Global Search Algorithm for Optimal Placement of micro-PMU in Distribution Networks". IEEE PES Innovative Smart Grid Technologies-Asia (ISGT-Asia), 559-563 (2016). 


\section{Bibliography}

[1] J. Chen, "Power system state estimation using phasor measurement units," 2013. [Online]. Available: http://uknowledge.uky.edu/ece_etds/35/

[2] T. Van Cutsem, J. Horward, and M. Ribbens-Pavella, "A two-level static state estimator for electric power systems," IEEE Trans. Power App. Syst., vol. PAS-100, no. 8, pp. 3722-3732, Aug. 1981.

[3] A. Melipoulos, Power System Modeling Analysis and Control. Marcel Dekker, 2004.

[4] M. Göl and A. Abur, "LAV based robust state estimation for systems measured by PMUs," IEEE Trans. Smart Grid, vol. 5, no. 4, pp. 1808-1814, Jul. 2014.

[5] X. Tai, Z. Lin, M. Fu, and Y. Sun, "A new distributed state estimation technique for power networks," in American Control Conference (ACC), 2013, Jun. 2013, pp. 3338-3343.

[6] A. Abur and A. Gómez-Expósito, Power system state estimation: Theory and implementation. CRC Press, 2004.

[7] A. Monticelli, State estimation in electric power systems: A generalized approach. NewYork: Springer, 1999.

[8] Y. F. Huang, S. Werner, J. Huang, N. Kashyap, and V. Gupta, "State estimation in electric power grids: Meeting new challenges presented by the 
requirements of the future grid," IEEE Signal Process. Mag., vol. 29, no. 5, pp. 33-43, Sep. 2012.

[9] A. Meliopoulos, B. Fardanesh, and S. Zelingher, "Power system state estimation: modeling error effects and impact on system operation," in System Sciences, 2001. Proceedings of the 34th Annual Hawaii International Conference on, Jan. 2001, pp. 682-690.

[10] D. S. Babu, K. Jamuna, and B. Aryanandiny, "Power system state estimationa review," ACEEE Int. J. on Electrical and Power Engineering, vol. 5, no. 1, pp. 10-18, 2014.

[11] A.-S. K. Pathan, The State of the Art in Intrusion Prevention and Detection. CRC Press, 2014.

[12] C. Muscas, M. Pau, P. A. Pegoraro, and S. Sulis, "Effects of measurements and pseudomeasurements correlation in distribution system state estimation," IEEE Trans. Instrum. Meas., vol. 63, no. 12, pp. 2813-2823, Dec. 2014.

[13] W. K. Ho, K. V. Ling, H. D. Vu, and X. Wang, "Filtering of the armax process with generalized t-distribution noise: The influence function approach," Industrial $\&$ Engineering Chemistry Research, vol. 53, no. 17, pp. 7019-7028, 2014.

[14] D. Wang and J. Romagnoli, "A framework for robust data reconciliation based on a generalized objective function," Industrial $\&$ engineering chemistry research, vol. 42, no. 13, pp. 3075-3084, 2003.

[15] V. Barnett and T. Lewis, Outliers in statistical data. Wiley, New York, 1994.

[16] S. Raghuraman and R. Jegatheesan, "A survey on state estimation techniques in electrical power system," in Recent Advancements in Electrical, Electronics 
and Control Engineering (ICONRAEeCE), 2011 International Conference on, Dec 2011, pp. 199-205.

[17] N. Shivakumar and A. Jain, "A review of power system dynamic state estimation techniques," in Power System Technology and IEEE Power India Conference, 2008. POWERCON 2008. Joint International Conference on, Oct. 2008, pp. 1-6.

[18] A. Monticelli, "Electric power system state estimation," Proceedings of the IEEE, vol. 88, no. 2, pp. 262-282, 2000.

[19] F. F. Wu, "Power system state estimation: a survey," Int. J. Elec. Power \&6 Energy Syst., vol. 12, no. 2, pp. 80-87, 1990.

[20] T. Van Cutsem and M. Ribbens-Pavella, "Bad data identification methods in power system state estimation: a comparative study," IEEE Trans. Power App. Syst., vol. 104, no. 11, 1985.

[21] A. Gómez-Expósito, A. Abur, A. de la Villa Jaen, and C. Gómez-Quiles, "A multilevel state estimation paradigm for smart grids," Proceedings of the IEEE, vol. 99, no. 6, pp. 952-976, Jun. 2011.

[22] L. Xie, D.-H. Choi, S. Kar, and H. Poor, "Fully distributed state estimation for wide-area monitoring systems," IEEE Trans. Smart Grid, vol. 3, no. 3, pp. 1154-1169, Sep. 2012.

[23] V. Kekatos and G. Giannakis, "Distributed robust power system state estimation," IEEE Trans. Power Syst., vol. 28, no. 2, pp. 1617-1626, May 2013.

[24] X. Li and A. Scaglione, "Robust decentralized state estimation and tracking for power systems via network gossiping," IEEE J. Select. Areas Commum., vol. 31, no. 7, pp. 1184-1194, Jul. 2013. 
[25] P. Huber, Robust statistics. Wiley, New York, 2009.

[26] F. Hampel, E. Ronchetti, P. Rousseeuw, and W. Stahel, Robust statistics: The approach based on influence functions. Wiley, 2011.

[27] R. Xiong and M. A. Grover, "A modified moving horizon estimator for in situ sensing of a chemical vapor deposition process," IEEE Transactions on Control Systems Technology, vol. 17, no. 5, pp. 1228-1235, Sept 2009.

[28] M. Farina, G. Ferrari-Trecate, and R. Scattolini, "Moving-horizon partitionbased state estimation of large-scale systems," Automatica, vol. 46, no. 5, pp. 910-918, 2010.

[29] M. Asprou, E. Kyriakides, and M. Albu, "The effect of variable weights in a wls state estimator considering instrument transformer uncertainties," IEEE Trans. Instrum. Meas., vol. 63, no. 6, pp. 1484-1495, Jun. 2014.

[30] H. Merrill and F. Schweppe, "Bad data suppression in power system static state estimation," IEEE Trans. Power App. Syst., vol. PAS-90, no. 6, pp. 2718-2725, Nov 1971.

[31] E. Handschin, F. Schweppe, J. Kohlas, and A. Fiechter, "Bad data analysis for power system state estimation," IEEE Trans. Power App. Syst., vol. 94, no. 2, pp. 329-337, Mar. 1975.

[32] R. Baldick, K. Clements, Z. Pinjo-Dzigal, and P. Davis, "Implementing nonquadratic objective functions for state estimation and bad data rejection," IEEE Trans. Power Syst., vol. 12, no. 1, pp. 376-382, Feb. 1997.

[33] M. Göl and A. Abur, "A robust PMU based three-phase state estimator using modal decoupling," IEEE Trans. Power Syst., vol. 29, no. 5, pp. 2292-2299, Sep. 2014. 
[34] G. Optimization et al., "Gurobi optimizer reference manual," URL: http://www. gurobi. com, vol. 2, pp. 1-3, 2012.

[35] R. Singh, B. C. Pal, and R. A. Jabr, "Choice of estimator for distribution system state estimation," IET generation, transmission $\&$ distribution, vol. 3, no. 7, pp. 666-678, July 2009.

[36] J. Zhao, M. Netto, and L. Mili, "A robust iterated extended kalman filter for power system dynamic state estimation," IEEE Trans. Power Syst., vol. 32, no. 99, pp. 3205-3216, Jul 2016.

[37] H. Yan, W. K. Ho, K. V. Ling, and K. W. Lim, "Multi-zone thermal processing in semiconductor manufacturing: Bias estimation," IEEE Trans. on Ind. Inform., vol. 6, no. 2, pp. 216-228, May 2010.

[38] J. J. Wang, S. B. Choy, and J. S. Chan, "Modelling stochastic volatility using generalized t distribution," Journal of Statistical Computation and Simulation, vol. 83, no. 2, pp. 340-354, 2013.

[39] S. Kotz and S. Nadarajah, Multivariate t-distributions and their applications. Cambridge University Press, 2004.

[40] G. Agamennoni, J. I. Nieto, and E. M. Nebot, "Approximate inference in statespace models with heavy-tailed noise," IEEE Trans. Signal Process., vol. 60, no. 10 , pp. 5024-5037, Oct 2012 .

[41] M. Roth, E. Özkan, and F. Gustafsson, "A student's t filter for heavy tailed process and measurement noise," in IEEE International Conference on Acoustics, Speech and Signal Processing, May 2013, pp. 5770-5774. 
[42] M. S. Aslam, "Maximum likelihood least squares identification method for active noise control systems with autoregressive moving average noise," Automatica, vol. 69, pp. 1-11, 2016.

[43] A. Mallet, "A maximum likelihood estimation method for random coefficient regression models," Biometrika, vol. 73, no. 3, pp. 645-656, 1986.

[44] D. Starer and A. Nehorai, "Newton algorithms for conditional and unconditional maximum likelihood estimation of the parameters of exponential signals in noise," IEEE Trans. Signal Process., vol. 40, no. 6, pp. 1528-1534, 1992.

[45] T. Brox, "Maximum likelihood estimation," in Computer Vision. Springer, 2014, pp. 481-482.

[46] A. Garcia, A. Monticelli, and P. Abreu, "Fast decoupled state estimation and bad data processing," IEEE Trans. Power App. Syst., vol. PAS-98, no. 5, pp. 1645-1652, Sep. 1979.

[47] O. Alsac, N. Vempati, B. Stott, and A. Monticelli, "Generalized state estimation," IEEE Trans. Power Syst., vol. 13, no. 3, pp. 1069-1075, Aug 1998.

[48] L. Zhang and A. Abur, "State estimator tuning for pmu measurements," in North American Power Symposium (NAPS), 2011, Aug. 2011, pp. 1-4.

[49] M. Göl and A. Abur, "A fast decoupled state estimator for systems measured by PMUs," IEEE Trans. Power Syst., vol. 30, no. 5, pp. 2766-2771, Sep. 2015.

[50] H. Merrill and F. Schweppe, "Bad data suppression in power system static state estimation," IEEE Trans. Power App. Syst., vol. PAS-90, no. 6, pp. 2718-2725, Nov 1971. 
[51] D. Falcao, P. Cooke, and A. Brameller, "Power system tracking state estimation and bad data processing," IEEE Trans. Power App. Syst., vol. PAS-101, no. 2, pp. 325-333, Feb 1982.

[52] G. Korres, "A robust algorithm for power system state estimation with equality constraints," IEEE Trans. Power Syst., vol. 25, no. 3, pp. 1531-1541, Aug. 2010.

[53] K. Clements, P. Davis, and K. Frey, "Treatment of inequality constraints in power system state estimation," IEEE Trans. Power Syst., vol. 10, no. 2, pp. 567-574, May 1995.

[54] A. Al-Othman and M. Irving, "A comparative study of two methods for uncertainty analysis in power system state estimation," IEEE Trans. Power Syst., vol. 20, no. 2, pp. 1181-1182, May 2005.

[55] M. Irving, "Robust algorithm for generalized state estimation," IEEE Trans. Power Syst., vol. 24, no. 4, pp. 1886-1887, Nov. 2009.

[56] C. V. Rao, J. B. Rawlings, and J. H. Lee, "Constrained linear state estimationa moving horizon approach," Automatica, vol. 37, no. 10, pp. 1619-1628, 2001.

[57] C. Rao, J. Rawlings, and D. Mayne, "Constrained state estimation for nonlinear discrete-time systems: stability and moving horizon approximations," IEEE Trans. Automat. Contr., vol. 48, no. 2, pp. 246-258, Feb. 2003.

[58] A. Alessandri, M. Baglietto, G. Battistelli, and V. Zavala, "Advances in moving horizon estimation for nonlinear systems," in Decision and Control (CDC), 2010 49th IEEE Conference on, Dec. 2010, pp. 5681-5688. 
[59] C. V. Rao, "Moving horizon strategies for the constrained monitoring and control of nonlinear discrete-time systems," Ph.D. dissertation, University of Wisconsin-Madison, 2000.

[60] G. C. Goodwin, M. M. Seron, and J. A. De Doná, Constrained control and estimation: an optimisation approach. Springer, 2006.

[61] T. Chen, T. Tran, D. Zhou, W. K. Ho, and K. V. Ling, "Iterated moving horizon estimation for power systems," in International Conference on Control, Automation and Information Sciences (ICCAIS), Dec. 2014, pp. 81-86.

[62] P. Philipp, "Centralized and distributed moving horizon strategies for state estimation of networked control systems." PhD thesis, Technischen Universität München, 2014.

[63] S. Boyd, N. Parikh, E. Chu, B. Peleato, and J. Eckstein, "Distributed optimization and statistical learning via the alternating direction method of multipliers," Foundations and Trends@ in Machine Learning, vol. 3, no. 1, pp. $1-122,2011$.

[64] J. Eckstein, "Splitting methods for monotone operators with applications to parallel optimization," Ph.D. dissertation, Massachusetts Institute of Technology, 1989.

[65] E. Ghadimi, "Accelerating convergence of large-scale optimization algorithms," Ph.D. dissertation, 2015.

[66] D. Boley, "Local linear convergence of the alternating direction method of multipliers on quadratic or linear programs," SIAM Journal on Optimization, vol. 23, no. 4, pp. 2183-2207, 2013. 
[67] T. V. Dang and K. V. Ling, "Moving horizon estimation on a chip," in 2014 13th International Conference on Control Automation Robotics Vision (ICARCV), Dec 2014, pp. 431-437.

[68] G. Wang, S.-J. Kim, and G. Giannakis, "Moving-horizon dynamic power system state estimation using semidefinite relaxation," in IEEE PES General Meeting - Conference Exposition, Jul. 2014, pp. 1-5.

[69] A. Gómez-Expósito, A. de la Villa Jaén, C. Gómez-Quiles, P. Rousseaux, and T. Van Cutsem, "A taxonomy of multi-area state estimation methods," Elect. Power Syst. Res., vol. 81, no. 4, pp. 1060-1069, 2011.

[70] R. Ebrahimian and R. Baldick, "State estimation distributed processing [for power systems]," IEEE Trans. Power Syst., vol. 15, no. 4, pp. 1240-1246, Nov. 2000.

[71] J. A. Aguado, C. Perez-Molina, and V. H. Quintana, "Decentralised power system state estimation: a decomposition-coordination approach," in Proc. IEEE Porto Power Tech Conf., vol. 3, Sep. 2001, pp. 10-13.

[72] M. Zhao and A. Abur, "Multi area state estimation using synchronized phasor measurements," IEEE Trans. Power Syst., vol. 20, no. 2, pp. 611-617, May 2005.

[73] G. Korres, "A distributed multiarea state estimation," IEEE Trans. Power Syst., vol. 26, no. 1, pp. 73-84, Feb. 2011.

[74] F. Pasqualetti, R. Carli, and F. Bullo, "Distributed estimation via iterative projections with application to power network monitoring," Automatica, vol. 48, no. 5, pp. 747-758, 2012. 
[75] A. J. Conejo, S. de la Torre, and M. Canas, "An optimization approach to multiarea state estimation," IEEE Trans. Power Syst., vol. 22, no. 1, pp. 213-221, Feb 2007.

[76] M. Farina, G. Ferrari-Trecate, and R. Scattolini, "Distributed moving horizon estimation for linear constrained systems," IEEE Trans. Automat. Contr., vol. 55, no. 11, pp. 2462-2475, Nov. 2010.

[77] A. Haber and M. Verhaegen, "Moving horizon estimation for large-scale interconnected systems," IEEE Trans. Automat. Contr., vol. 58, no. 11, pp. 2834-2847, Nov. 2013.

[78] J. Zhang and J. Liu, "Distributed moving horizon state estimation with triggered communication," in American Control Conference (ACC), Jun. 2014, pp. $5700-5705$.

[79] V. Terzija, G. Valverde, D. Cai, P. Regulski, V. Madani, J. Fitch, S. Skok, M. M. Begovic, and A. Phadke, "Wide-area monitoring, protection, and control of future electric power networks," Proceedings of the IEEE, vol. 99, no. 1, pp. 80-93, Jan 2011.

[80] A. Bose, A. Abur, K. Y. K. Poon, and R. Emami, "Implementation issues for hierarchical state estimators," Final Project Report, pp. 10-11, 2010.

[81] J. Zhang and J. Liu, "Distributed moving horizon state estimation for nonlinear systems with bounded uncertainties," Journal of Process Control, vol. 23, no. 9, pp. 1281-1295, 2013.

[82] J. Zeng and J. Liu, "Distributed moving horizon state estimation: Simultaneously handling communication delays and data losses," Systems $\&$ Control Letters, vol. 75, pp. 56-68, 2015. 
[83] R. Schneider, H. Scheu, and W. Marquardt, "An iterative partition-based moving horizon estimator for large-scale linear systems," in Proc. of the 12th European Control Conf., 2013, pp. 2621-2626.

[84] M. Ahmad, Power system state estimation. Artech House, 2012.

[85] J. Machowski, J. Bialek, and J. Bumby, Power system dynamics: Stability and control. John Wiley \& Sons, 2011.

[86] J. Zhang, G. Welch, and G. Bishop, "Observability and estimation uncertainty analysis for PMU placement alternatives," in Proc. North Amer. Power Symp., 2010, pp. 1-8.

[87] S. Chakrabarti and E. Kyriakides, "PMU measurement uncertainty considerations in WLS state estimation," IEEE Trans. Power Syst., vol. 24, no. 2, pp. 1062-1071, May 2009.

[88] G. He, S. Dong, J. Qi, and Y. Wang, "Robust state estimator based on maximum normal measurement rate," IEEE Trans. Power Syst., vol. 26, no. 4, pp. 2058-2065, Nov. 2011.

[89] L. Mili, M. Cheniae, N. Vichare, and P. Rousseeuw, "Robust state estimation based on projection statistics," IEEE Trans. Power Syst., vol. 11, no. 2, pp. 1118-1127, May 1996.

[90] L. C. Andrews, Special functions for engineers and applied mathematicians. Macmillan, 1985.

[91] P. Yang, Z. Tan, A. Wiesel, and A. Nehora, "Power system state estimation using PMUs with imperfect synchronization," IEEE Trans. Power Syst., vol. 28, no. 4, pp. 4162-4172, Nov. 2013. 
[92] I. Dabbagchi and R. Christie, "Power systems test case archive," University of Washington, Tech. Rep., 1993. [Online]. Available: http://www.ee.washington.edu/research/pstca/

[93] A. Gómez-Expósito, A. Abur, P. Rousseaux, A. de la Villa Jaén, and C. Gómez-Quiles, "On the use of PMUs in power system state estimation," in Proc. 17th Power Systems Computation Conference, vol. 22, 2011, p. 26.

[94] D. Peel and G. J. McLachlan, "Robust mixture modelling using the t distribution," Statistics and computing, vol. 10, no. 4, pp. 339-348, 2000.

[95] B. Grigelionis, Student's T-Distribution and Related Stochastic Processes. Springer, 2013.

[96] D. G. Robertson and J. H. Lee, "On the use of constraints in least squares estimation and control," Automatica, vol. 38, no. 7, pp. 1113-1123, 2002.

[97] Q. Wang, S. Li, X. Zhao, M. Wang, and S. Sun, "Wideband millimeterwave channel characterization based on los measurements in an open office at 26ghz," in IEEE 83rd Vehicular Technology Conference (VTC Spring), May 2016, pp. 1-5.

[98] C. Dufour and J. Bélanger, "On the use of real-time simulation technology in smart grid research and development," IEEE Trans. Ind. Applicat., vol. 50, no. 6, pp. 3963-3970, Nov. 2014.

[99] D. Bian, M. Kuzlu, M. Pipattanasomporn, S. Rahman, and Y. Wu, "Realtime co-simulation platform using opal-rt and opnet for analyzing smart grid performance," in IEEE Power Energy Society General Meeting, Jul. 2015, pp. $1-5$. 
[100] D. Gao, Y. Sun, and Y. Lu, "A robust demand response control of commercial buildings for smart grid under load prediction uncertainty," Energy, vol. 93, pp. 275-283, 2015.

[101] Y. Liu, F. Shang, W. Fan, J. Cheng, and H. Cheng, "Generalized higher-order orthogonal iteration for tensor decomposition and completion," in Advances in Neural Information Processing Systems, 2014, pp. 1763-1771.

[102] D. G. Robertson, J. H. Lee, and J. B. Rawlings, "A moving horizon-based approach for least-squares estimation," AIChE Journal, vol. 42, no. 8, pp. 2209-2224, 1996.

[103] P. K. Findeisen, "Moving horizon state estimation of discrete time systems." Master thesis, University of Wisconsin-Madison, 1997.

[104] C. V. Rao and J. B. Rawlings, "Constrained process monitoring: Movinghorizon approach," AIChE journal, vol. 48, no. 1, pp. 97-109, 2002.

[105] J. Eckstein, "Parallel alternating direction multiplier decomposition of convex programs," Journal of Optimization Theory and Applications, vol. 80, no. 1, pp. 39-62, 1994.

[106] J. Eckstein and M. C. Ferris, "Operator-splitting methods for monotone affine variational inequalities, with a parallel application to optimal control," INFORMS Journal on Computing, vol. 10, no. 2, pp. 218-235, 1998.

[107] L. Hu, Z. Wang, I. Rahman, and X. Liu, "A constrained optimization approach to dynamic state estimation for power systems including pmu and missing measurements," IEEE Transactions on Control Systems Technology, vol. 24, no. 2, pp. 703-710, March 2016. 
[108] A. Abur and M. Celik, "Least absolute value state estimation with equality and inequality constraints," IEEE Trans. Power Syst., vol. 8, no. 2, pp. 680686, May 1993.

[109] A. Tarali and A. Abur, "Bad data detection in two-stage state estimation using phasor measurements," in 2012 3rd IEEE PES Innovative Smart Grid Technologies Europe (ISGT Europe), Oct 2012, pp. 1-8.

[110] J. Zhao, G. Zhang, and M. L. Scala, "A two-stage robust power system state estimation method with unknown measurement noise," in 2016 IEEE Power and Energy Society General Meeting (PESGM), July 2016, pp. 1-5.

[111] T. Chen, D. Zhou, T. Tran, C. Kastner, K. V. Ling, K. J. Tseng, and J. M. Maciejowski, "Distributed moving horizon estimation for power systems," in IEEE Power Energy Society General Meeting, Jul. 2015, pp. 1-5.

[112] F. Chung, Spectral Graph Theory. American Mathematical Society, 1997.

[113] B. Bollobás, Modern Graph Theory. Springer, 1998.

[114] S. Kar and J. Moura, "Convergence rate analysis of distributed gossip (linear parameter) estimation: Fundamental limits and tradeoffs," IEEE J. Sel. Topics in Signal Process., vol. 5, no. 4, pp. 674-690, Aug. 2011.

[115] S. Kar, J. Moura, and K. Ramanan, "Distributed parameter estimation in sensor networks: Nonlinear observation models and imperfect communication," IEEE Trans. Inf. Theory,, vol. 58, no. 6, pp. 3575-3605, Jun. 2012.

[116] Y. Sun, M. Fu, B. Wang, and H. Zhang, "A distributed map approach to dynamic state estimation with applications in power networks," in 2015 European Control Conference (ECC), July 2015, pp. 235-240. 
[117] W. Zhang, W. Liu, C. Zang, and L. Liu, "Multiagent system-based integrated solution for topology identification and state estimation," IEEE Transactions on Industrial Informatics, vol. 13, no. 2, pp. 714-724, April 2017.

[118] F. Aminifar, M. Shahidehpour, M. Fotuhi-Firuzabad, and S. Kamalinia, "Power system dynamic state estimation with synchronized phasor measurements," IEEE Trans. Instrum. Meas., vol. 63, no. 2, pp. 352-363, Feb. 2014.

[119] K. J. Åström and B. Wittenmark, Adaptive control. Courier Corporation, 2013.

[120] A. Monticelli and A. Garcia, "Reliable bad data processing for real-time state estimation," IEEE Trans. Power App. Syst., vol. PAS-102, no. 5, pp. 11261139, May 1983. 


\section{Appendix A}

\section{Weighted Least Squares Algorithm}

Considering the nonlinear measurement model,

$$
y_{k}=h\left(x_{k}\right)+v_{k}
$$

where $y_{k}$ is the measurement vector, $x_{k}$ is the state vector and $v_{k}$ is the Gaussian noise assumption.

Denote that matrix $W$ is a positive definite matrix if $\forall x \neq 0, x^{T} W x>0$.

According to literature $[6,7]$, the PSSE problem can be defined as a minimizing equation

$$
J\left(x_{k}\right)=\left[y_{k}-h\left(x_{k}\right)\right]^{T} R^{-1}\left[y_{k}-h\left(x_{k}\right)\right] .
$$

where $R=\operatorname{diag}\left\{\sigma_{1}^{2}, \sigma_{2}^{2}, \cdots, \sigma_{m}^{2}\right\}$ and $\sigma_{i}$ is the standard deviation of measurement $i$.

If the approximated linear model of $h\left(x_{k}\right)=H x_{k}$ is employed in $(A .1)$, the SE problem is in the form of

$$
J\left(x_{k}\right)=e_{k}^{T} R^{-1} e_{k},
$$

in which $R$ is a positive definite matrix and $e_{k}$ is the measurement residual as given by

$$
e_{k}=y_{k}-H x_{k}
$$


The first-order optimal condition for $x_{k}$ is then obtained from the following:

$$
\begin{aligned}
\frac{\partial J\left(x_{k}\right)}{\partial x_{k}} & =2 H^{T} R^{-1} H x_{k}-2 H^{T} R^{-1} y_{k} \\
& =-2 H^{T} R^{-1}\left(y_{k}-H x_{k}\right)=0 .
\end{aligned}
$$

Solving the normal equation gives

$$
x_{k}=G^{-1} H^{T} R^{-1} y_{k},
$$

where $G$ is designated as the gain matrix and is given as

$$
G=H^{T} R^{-1} H
$$

At the minimum, the first-order optimality conditions will have to be satisfied. These can be expressed in compact form as given by [6]

$$
g\left(x_{k}\right)=\frac{\partial J\left(x_{k}\right)}{\partial x_{k}}=-\left(H\left(x_{k}\right)\right)^{T} R^{-1}\left[y_{k}-h\left(x_{k}\right)\right]=0
$$

where $H\left(x_{k}\right)$ is the $m \times n$ Jacobian matrix and is given by

$$
H\left(x_{k}\right)=\left.\frac{\partial h(x)}{\partial x}\right|_{x=x_{k}}
$$

Expanding the non-linear function $g\left(x_{k}\right)$ into Taylor series at state vector $x_{k}^{q}$,

$$
g\left(x_{k}\right)=g\left(x_{k}^{q}\right)+G\left(x_{k}^{q}\right)\left(x_{k}-x_{k}^{q}\right)+\cdots=0
$$

where $q$ is the iteration index. Taking only the first-order Taylor series into account, 
an iterative solution scheme known as the Gauss-Newton method is given by:

$$
\begin{aligned}
x_{k}^{q+1} & =x_{k}^{q}-\left[G\left(x_{k}^{q}\right)\right]^{-1} g\left(x_{k}^{q}\right) \\
& =x_{k}^{q}+\left[G\left(x_{k}^{q}\right)\right]^{-1} H^{T}\left(x_{k}^{q}\right) R^{-1}\left(y_{k}-h\left(x_{k}^{q}\right)\right)
\end{aligned}
$$

where $x_{k}^{q}$ is the solution vector at iteration $q$ and the gain matrix is $G\left(x_{k}^{q}\right)=$ $\left(H\left(x_{k}^{q}\right)\right)^{T} R^{-1} H\left(x_{k}^{q}\right)$.

The above iteration will be terminated when the difference $\Delta x_{k}^{q}=\left(x_{k}^{q+1}-x_{k}^{q}\right)$ reaches a fixed threshold, e.g., say $\delta_{W L S}$. 


\section{Appendix B}

\section{Influence Function}

The Bayesian estimation is based on theories of the classical parametric statistics and an optimal procedure under exact parametric models. In order to explore the performance of estimation when the models are only approximately valid (or, say the performance of the estimation in the neighborhood of the assumed parametric model), Hampel [26] proposes the influence function based on the pioneer work done by Huber in [25] where a rather full kind of neighborhood of a strict parametric model was considered for the first time. The theory of influence function provides a framework for judging and comparing the existing statistical procedures under the aspect of robustness, and finally develops and suggests new robust procedures.

Suppose $m$ one-dimensional observations $Y_{1}, \ldots, Y_{m}$ are independent and identically distributed (i.i.d). The observations belong to some sample space and a parametric model consists of a family of probability distributions $F_{\theta}$ is on the sample space, where the unknown parameter $\theta$ belongs to some parameter space and this parameter space is an open convex subset. As estimators of $\theta$, consider the real value statistics (estimators)

$$
\mathbf{T}_{m}=\mathbf{T}_{m}\left(Y_{1}, \ldots, Y_{m}\right)=\mathbf{T}_{m}\left(G_{m}\right)
$$


where $G_{m}$ is the empirical distribution of the observations. Formally $G_{m}$ is given by $\frac{1}{m} \sum_{i=1}^{m} \Delta_{\iota_{i}}, \iota$ is on the sample space where this limit exists and $\Delta_{\iota}$ is the point mass 1 in $Y$.

In a broader sense, an estimator can be viewed as a sequence of statistics $\left\{\mathbf{T}_{m} ; m \geq\right.$ $1\}$, one for each possible sample size $m$. We consider estimators which are functionals, i.e., $\mathbf{T}_{m}\left(G_{m}\right)=\mathbf{T}\left(G_{m}\right)$ for all $m$ and $G_{m}$, or can asymptotically be replaced by functionals. It is assumed that the functionals under study are Fisher consistent:

$$
\mathbf{T}\left(F_{\theta}\right)=\theta \text { for all } \theta
$$

which means that at the model, i.e., the observations are independent and identically distributed (i.i.d) according to the true distribution $F_{\theta}$ in domain $(\mathbf{T})$, and the estimator $\left\{\mathbf{T}_{m} ; m \geq 1\right\}$ asymptotically measures the right quantity. Assume that $\mathbf{T}$ is Gâteaux differentiable at distribution $F$ in domain $(\mathbf{T})$, the influence function is expressed as a definition:

Definition B.0.1. The Influence Function (IF) [26] of $\mathbf{T}$ at $F$ is given by

$$
\operatorname{IF}(\iota ; \mathbf{T}, F)=\lim _{\eta \rightarrow 0} \frac{\mathbf{T}\left((1-\eta) F+\eta \Delta_{\iota}\right)-\mathbf{T}(F)}{\eta}
$$

where $\eta \in[0,1]$ is the mass of contamination to $F ; \iota$ is on the sample space where this limit exists; and $\Delta_{\iota}$ is the probability measure which puts point mass 1 at the point $\iota$.

The importance of the IF lies in its heuristic interpretation: it describes the effect of an infinitesimal contamination at the point $\iota$ on the estimate, standardized by the mass of the contamination. If we say that the point $\iota$ is an outlier the IF indeed describes the effect of an outlier on the estimation [26]. Someone can also understand that the IF measures the asymptotic bias caused by contamination in 
the observations. Thus from the IF point of view, the IF of a robust estimator should be bound and/or descending, or increasing slowly for large magnitude of noise (also remarked in [119] the robust estimator may be obtained by mapping the estimation error such that the nonlinear map function is linear for small estimation error but increases more slowly than linear for large estimation error).

Now let us focus on the important relationship between the IF and asymptotic variance. When the observations $Y_{i}$ are i.i.d. according to $F$, the empirical distribution $F_{m}$ will tend to $F$ by the Glivenko-Cantelli theorem [26]. The distribution of $\sqrt{m}\left(\mathbf{T}_{m}-\mathbf{T}(F)\right)$ tends to normal distribution $N(0, \operatorname{Var}(\mathbf{T}, F))$ as $m$ goes to infinity, where the asymptotic variance equals

$$
\operatorname{Var}(\mathbf{T}, F)=\int_{-\infty}^{\infty} I F(\iota ; \mathbf{T}, F)^{2} d F(\iota)
$$

To obtain the IF of the robust estimator in Chapter 3, let the cumulative probability distribution of the measurement noise vector $\varepsilon=Z-\bar{H} x$ be $F(\varepsilon)$, where $Z$ is the measurement vector; $\bar{H}$ is the measurement matrix and $x$ is the true state vector. The robust estimator provides an estimate of the state by processing the redundant observation vector $Z$ and solving the following equation:

$$
\Psi(\varepsilon)=0
$$

where $\Psi$ is the derivative of the cost function, as given in Chapter 3. By taking expectation, equation (B.3) can be written as

$$
\int_{-\infty}^{\infty} \Psi(\varepsilon) f(\varepsilon) d \varepsilon=0
$$

To study the change in $x$ when the distribution changes from $f(\varepsilon)$ to a new distribution $f_{0}(\varepsilon)$, replace $f(\varepsilon)$ in (B.4) by $(1-\eta) f(\varepsilon)+\eta f_{0}(\varepsilon)$, where $\eta \in[0,1]$. This 
yields

$$
\int_{-\infty}^{\infty} \Psi(\varepsilon)\left((1-\eta) f(\varepsilon)+\eta f_{0}(\varepsilon)\right) d \varepsilon=0
$$

Equation (B.5) implicitly defines the estimation as a function of $\eta$, and it is denoted as $\mathbf{T}(\eta)$. According to the definition of the Influence Function (B.1), the IF of the robust estimator can be obtained by differentiating (B.5) and setting $\eta=0$

$$
\left.\mathrm{IF}(\varepsilon) \triangleq \frac{d \mathbf{T}(\eta)}{d \eta}\right|_{\eta=0}
$$

Differentiating (B.5) with respect to $\eta$ gives

$$
\frac{\partial}{\partial \eta} \int_{-\infty}^{\infty} \Psi(\varepsilon)\left((1-\eta) f(\varepsilon)+\eta f_{0}(\varepsilon)\right) d \varepsilon=0
$$

that is,

$$
\begin{aligned}
& \int_{-\infty}^{\infty} \Psi(\varepsilon)\left(-\eta f(\varepsilon)+f_{0}(\varepsilon)\right) d \varepsilon \\
& +\left(\int_{-\infty}^{\infty} \frac{\partial \Psi(\varepsilon)}{\partial \mathbf{T}(\eta)}\left((1-\eta) f(\varepsilon)+\eta f_{0}(\varepsilon)\right) d \varepsilon\right) \frac{\partial \mathbf{T}(\eta)}{\partial \eta}=0
\end{aligned}
$$

Let $\eta=0$ and use (B.4), equation (B.7) reduces to

$$
\left.\frac{\partial \mathbf{T}(\eta)}{\partial \eta}\right|_{\eta=0}=-\left[\int_{-\infty}^{\infty} \frac{\Psi(\varepsilon)}{\partial x} f(\varepsilon) d \varepsilon\right]^{-1} \int_{-\infty}^{\infty} \Psi(\varepsilon) f_{0}(\varepsilon) d \varepsilon
$$

When $\eta=0$, the associated probability density function of $x$ is $f(\varepsilon)$ and the initial state is at the operating point $x=\bar{x}$. Let $f_{0}(\varepsilon)$ be an impulse function at $\varepsilon$ and 
equation (B.8) reduces to the influence function

$$
\begin{aligned}
\operatorname{IF}(\varepsilon) & =\left.\frac{\partial \mathbf{T}(\eta)}{\partial \eta}\right|_{\eta=0} \\
& =-\left.\left[\int_{-\infty}^{\infty} \frac{\Psi(\varepsilon)}{\partial x} f(\varepsilon) d \varepsilon\right]^{-1} \Psi(\varepsilon)\right|_{x=\bar{x}}
\end{aligned}
$$

The Taylor series expansion of $\mathbf{T}(\eta)$ at $\eta=0$ is given by

$$
\mathbf{T}(\eta)=\bar{x}+\left.\frac{d \mathbf{T}(\eta)}{d \eta}\right|_{\eta=0} \eta+\left.\frac{1}{2} \frac{d^{2} \mathbf{T}(\eta)}{d \eta^{2}}\right|_{\eta=0} \eta^{2}+\cdots
$$

Since (B.5) reduces to (B.3) when $\eta=1, \mathbf{T}(1)$ is the solution of $x$. We can get an approximate solution to this by retaining the first-order Taylor series at operating point $x=\bar{x}$. This results in

$$
\hat{x} \approx \bar{x}+\operatorname{IF}(\varepsilon)
$$

which is the same as (3.18). 


\section{Appendix C}

\section{WLS with Largest Normalized \\ Residuals}

WLS is not a robust estimator. However, the largest normalized residuals (LNR) method [120] is usually associated with the WLS estimator to process bad data. The normalized residuals are calculated as:

$$
\begin{aligned}
\bar{R} & =\operatorname{diag}\left(\sigma_{1}^{2}, \ldots, \sigma_{m}^{2}, \ldots, \sigma_{1}^{2}, \ldots, \sigma_{m}^{2}\right) \in \mathbb{R}^{(N+1) m \times(N+1) m}, \\
\bar{G} & =\bar{H}^{T} \bar{R}^{-1} \bar{H} \\
\bar{\Omega} & =\bar{R}-\bar{H} \bar{G}^{-1} \bar{H}^{T} \\
e_{i, k}^{\text {norm }} & =\frac{\left|e_{i, k}\right|}{\sqrt{\bar{\Omega}_{i i}}} .
\end{aligned}
$$

As given in (C.1), the normalized residuals $e_{i, k}^{\text {norm }}$ are calculated according to the residual covariance matrix $\bar{\Omega}$ and measurement residual $e_{i, k}$. If the normalized residuals $e_{i, k}^{n o r m}$ are larger than a pre-determined threshold, for example, 3.0, the largest one will correspond to the bad measurement. Once the LNR is found, the 
corresponding measurement is updated by

$$
Z_{i}^{\text {new }}=Z_{i}^{b a d}-\frac{\bar{R}_{i i}}{\bar{\Omega}_{i i}} e_{i, k}^{b a d}
$$

The states will then be recalculated based on the updated measurements. Several iterations may be needed in order to make sure that all normalized residuals are less than a pre-determined threshold. 